\title{
Corey-Chaykovsky Cyclopropanation of Nitronaphthalenes: Access to Benzonorcaradienes and Related Systems
}

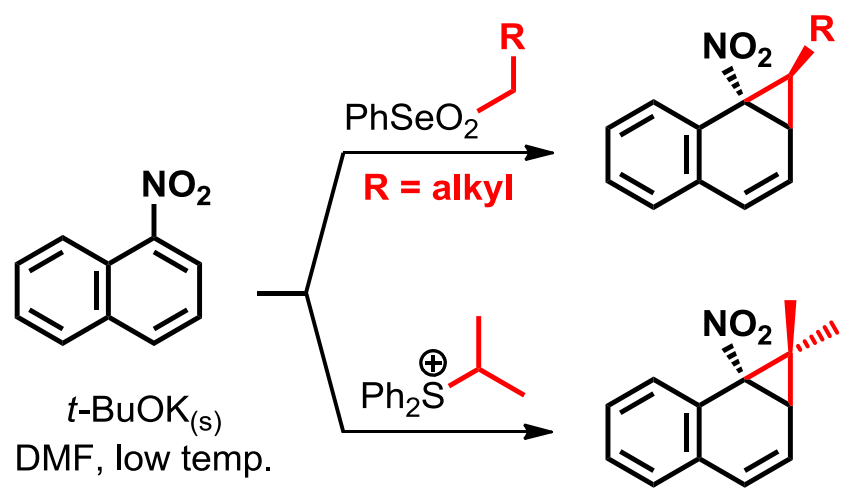




\section{Table of content}

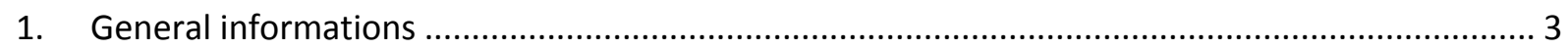

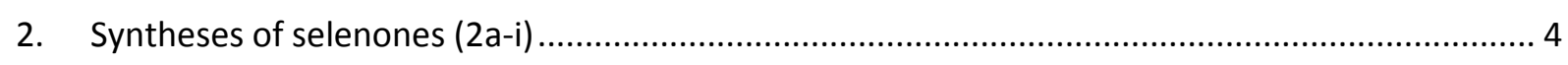

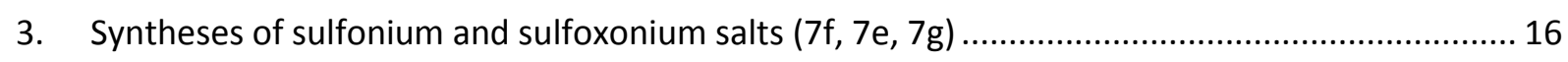

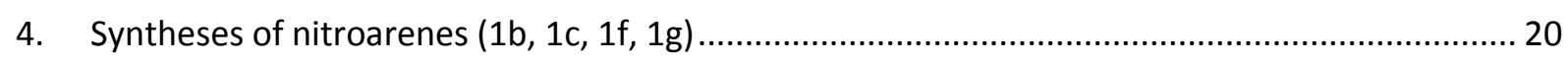

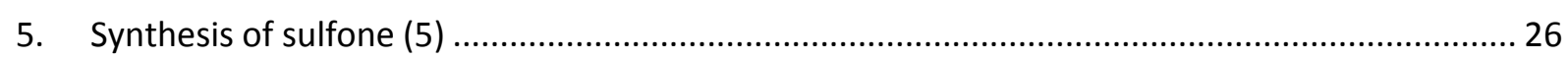

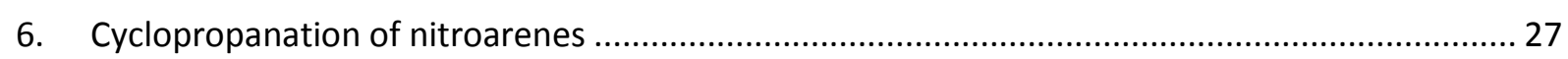

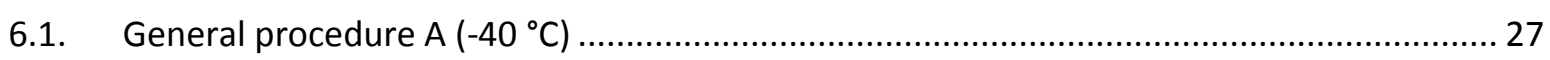

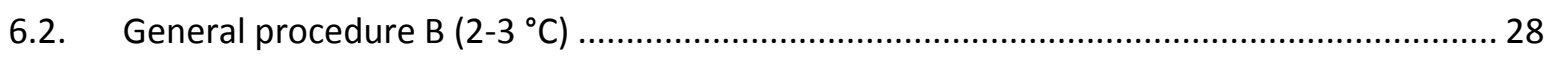

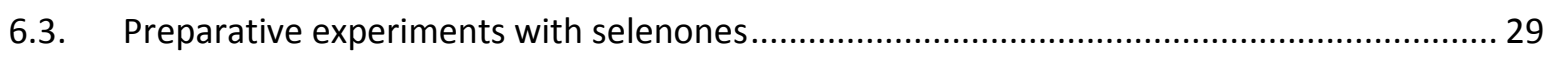

6.4. Preparative experiments with sulfonium salts........................................................ 30

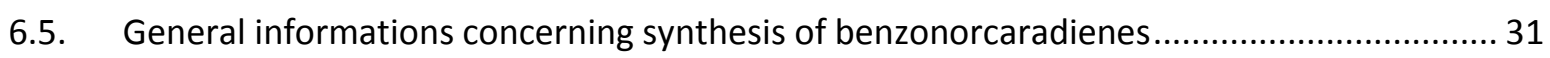

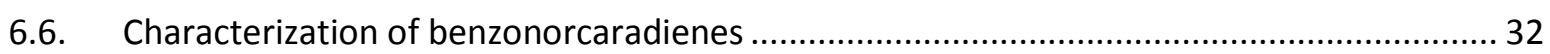

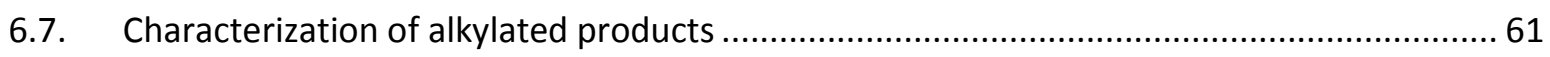

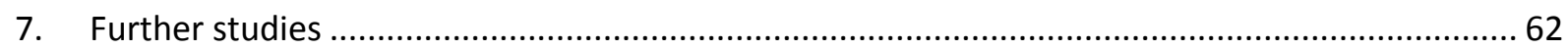

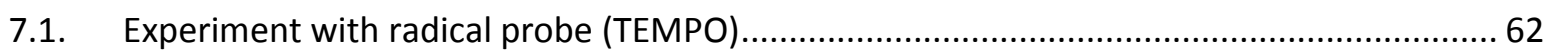

7.2. NMR signals assignment for endo- and exo-benzonorcaradienes ...................................63 63

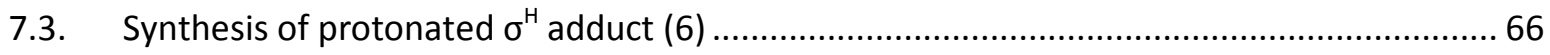

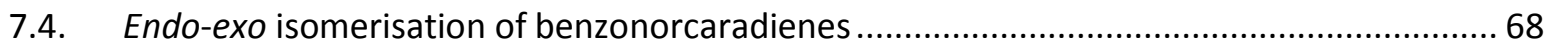

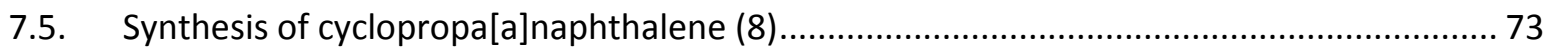

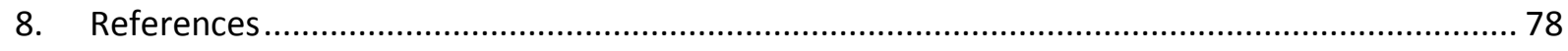

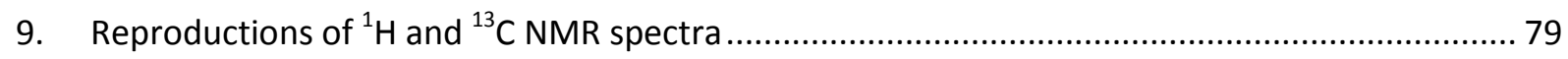




\section{General informations}

Thiophenol, ethyl bromide, octyl bromide, isobutyl bromide, isopropyl bromide, neopentyl alcohol, sodium borohydride, $m C P B A$, trimethyl sulfonium bromide, trimethyl sulfoxonium iodide, TEMPO, 5-nitroindazole, potassium tert-butoxide, $\mathrm{FeCl}_{2} \cdot 4 \mathrm{H}_{2} \mathrm{O}$ and DMF (anhydrous) were purchased from Sigma-Aldrich.

Methyl iodide was purchased from Acros.

Methyldiphenylsulfonium tetrafluoroborate and triethylsulfonium iodide were purchased from Alfa Aesar.

Methanesulfonyl chloride and butyl iodide were purchased from Fluka.

Diphenyl diselenide, 1-bromo-2-ethylbutane, cyclohexylmethyl bromide, cyclobutylmethyl bromide, 2-nitronaphthalene, 6-nitroquinoline, silver tetrafluoroborate and KHMDS (20\% w/w solution in THF) were purchased from Fluorochem.

1-Nitronaphthalene was purchased from Merck.

Phenyl sulfide was purchased form TCl.

1-Naphthol was purchased from FOC Gliwice, Poland.

Triethylamine was purchased from POCh, Poland.

Acetic anhydride, concentrated $\mathrm{HNO}_{3}, \mathrm{KOH}, \mathrm{K}_{2} \mathrm{CO}_{3}$ and $\mathrm{Na}_{2} \mathrm{CO}_{3}$ were purchased from ChemPur, Poland.

Commercially available solvents and materials were used without further purification.

Column chromatography was performed on silica gel (high-purity grade, pore size $60 \AA$, $230-400$ mesh particle size, 40-63 m, 60737).

Thin layer chromatography (TLC) was performed on Supelco silica gel on TLC Al foils with fluorescent indicator $254 \mathrm{~nm}$ and was visualized under UV lamp.

Analytical GLC was performed on a PerkinElmer Clarus 580 chromatograph equipped with a flame ionization detector, and a GL Sciences InertCap 5MS/Sil column with He as a carrier gas (column 0.25 $\mathrm{mm} \times 30 \mathrm{~m}$, carrier flow $1.5 \mathrm{~mL} / \mathrm{min}$, method parameters $50{ }^{\circ} \mathrm{C},+10^{\circ} \mathrm{C} / \mathrm{min}$ to $300{ }^{\circ} \mathrm{C}$, then $15 \mathrm{~min}$ at $\left.300^{\circ} \mathrm{C}\right)$.

${ }^{1} \mathrm{H},{ }^{19} \mathrm{~F}$, and ${ }^{13} \mathrm{C}$ NMR spectra were recorded on Agilent $400 \mathrm{MHz}$ NMR spectrometer. Chemical shifts $(\delta)$ are given in parts per million (ppm) with the solvent resonance as the internal standard (for $\mathrm{CDCl}_{3}$ : 7.24 and $77.0 \mathrm{ppm}$, for ${ }^{1} \mathrm{H}$ and ${ }^{13} \mathrm{C}$ NMR, respectively) or with $\mathrm{CFCl}_{3}$ (0.0 ppm for ${ }^{19} \mathrm{~F} \mathrm{NMR}$ ). Spin multiplicity was abbreviated as follows: $s$, singlet, $d$, doublet, $t$, triplet, $q$, quartet, $p$, pentet, hept, heptet, $\mathrm{m}$, multiplet.

Melting points were uncorrected.

All crystals suitable for X-Ray studies were grown by slow evaporation of each compounds solution in dichloromethane/heptane mixture. 


\section{Syntheses of selenones (2a-i)}

All selenones were synthesized in two steps. Alkylation step was performed according to the literature. ${ }^{[1]}$ Oxidation step was done according to modified procedure described for oxidation of selenides to selenoxides. ${ }^{[2]}$

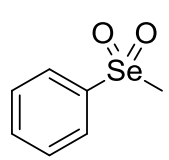

2a

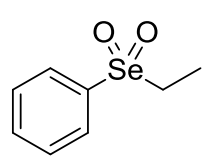

2b

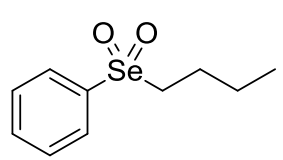

2c

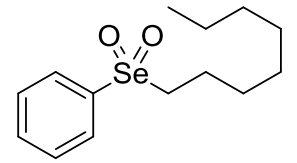

2d<smiles>CC(C)CS(=O)(=O)c1ccccc1</smiles>

2e<smiles>CCC(CC)C[Se](=O)(O)c1ccccc1</smiles>

$2 f$<smiles>CC(C)(C)C[Se](=O)(O)c1ccccc1</smiles>

2g<smiles>O=[Se](O)(CC1CCC1)c1ccccc1</smiles>

2h<smiles>O=[Se](O)(CC1CCCCC1)c1ccccc1</smiles>

2i 


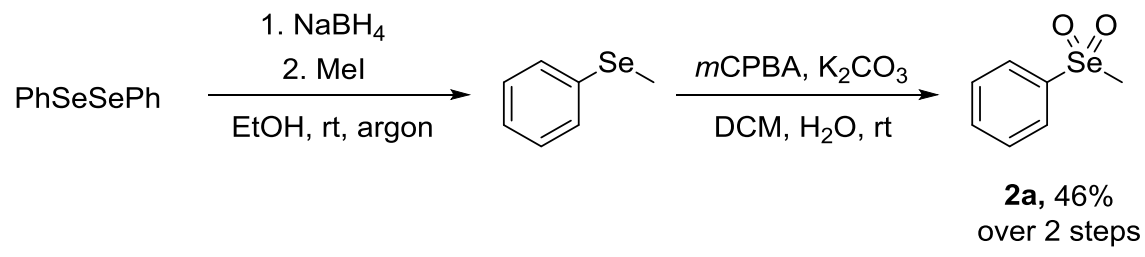

A $250 \mathrm{~mL}$ round-bottom flask was charged with diphenyl diselenide $(9.29 \mathrm{~g}, 29.8 \mathrm{mmol}, 1.0$ equiv.), flushed with argon and immersed in water bath (rt). Absolute ethanol $(80 \mathrm{~mL})$ was added and $\mathrm{NaBH}_{4}$ ( $3.38 \mathrm{~g}, 89.0 \mathrm{mmol}, 3.0$ equiv.) was added potionwise. After $45 \mathrm{~min}$ methyl iodide $(10.7 \mathrm{~g}, 75.0 \mathrm{mmol}$, 2.5 equiv.) was added dropwise. After $20 \mathrm{~h}$ reaction was quenched with $\mathrm{NH}_{4} \mathrm{Cl}_{\text {aq }}(100 \mathrm{~mL}, 10 \% \mathrm{w} / \mathrm{v})$. Resulted mixture was extracted with ethyl acetate $(3 \times 100 \mathrm{~mL})$, combined organic phases were washed with $\mathrm{H}_{2} \mathrm{O}(100 \mathrm{~mL})$, brine $(100 \mathrm{~mL})$, and dried over anhydrous $\mathrm{MgSO}_{4}$. Mixture was filtered and evaporated. Crude methyl phenyl selenide $(4.97 \mathrm{~g}, 29.1 \mathrm{mmol}, 1.0$ equiv., from $9.43 \mathrm{~g}, 55.1$ mmol obtained) was placed in $1 \mathrm{~L}$ round-bottom flask, dichloromethane $(200 \mathrm{~mL})$, and $\mathrm{K}_{2} \mathrm{CO}_{3}$ aq $(200$ $\mathrm{mL}$, sat.) were added, and to mechanically stirred mixture $m$ CPBA $(19.8 \mathrm{~g}, 88.2 \mathrm{mmol}$, ca. $77 \%$ purity, 3.0 equiv.) was added portionwise. After $2 \mathrm{~h} \mathrm{H}_{2} \mathrm{O}(200 \mathrm{~mL})$ was added, phases were separated, aqueous phase was extracted with dichloromethane $(2 \times 200 \mathrm{~mL})$, combined organic phases were washed with $\mathrm{H}_{2} \mathrm{O}(200 \mathrm{~mL})$, brine $(200 \mathrm{~mL})$, and dried over anhydrous $\mathrm{MgSO}_{4}$. Mixture was filtered, evaporated and separated with column chromatography (eluent: dichloromethane to dichloromethane/methanol 9:1) yielding 2a ( $2.97 \mathrm{~g}, 14.6 \mathrm{mmol}, \mathbf{4 6 \%}$ over $\mathbf{2}$ steps) as white crystals.<smiles>C[Sb](=O)(=O)c1ccccc1</smiles>

methyl phenyl selenone, $2 \mathrm{a}$

white crystals, $\mathrm{mp} 95.0^{\circ} \mathrm{C}$ (dec.)

eluent: dichloromethane to dichloromethane/methanol 9:1

$\mathrm{R}_{\mathrm{f}}=0.50$ (dichloromethane/methanol 9:1)

${ }^{1} \mathrm{H}$ NMR $\left(400 \mathrm{MHz}, \mathrm{CDCl}_{3}\right): \delta=8.02-7.96(\mathrm{~m}, 2 \mathrm{H}), 7.73-7.68(\mathrm{~m}, 1 \mathrm{H})$, $7.67-7.60(\mathrm{~m}, 2 \mathrm{H}), 3.27(\mathrm{~s}, 3 \mathrm{H})$.

${ }^{13} \mathrm{C}$ NMR $\left(100 \mathrm{MHz}, \mathrm{CDCl}_{3}\right): \delta=142.4,134.4,130.3,126.5,44.2$.

HRMS (ESI): $\mathrm{m} / \mathrm{z}[\mathrm{M}+\mathrm{Na}]^{+}$calcd for $\mathrm{C}_{7} \mathrm{H}_{8} \mathrm{O}_{2} \mathrm{SeNa}: 226.9587$; found: 226.9582. 


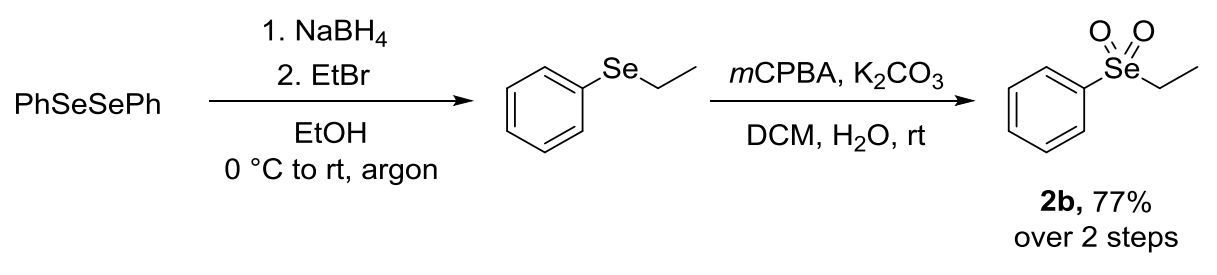

A $250 \mathrm{~mL}$ round-bottom flask was charged with diphenyl diselenide $(6.22 \mathrm{~g}, 19.9 \mathrm{mmol}, 1.0$ equiv.), flushed with argon and immersed in ice/water bath (ca. $\left.0{ }^{\circ} \mathrm{C}\right)$. Absolute ethanol $(60 \mathrm{~mL})$ was added and $\mathrm{NaBH}_{4}(2.36 \mathrm{~g}, 62.4 \mathrm{mmol}, 3.1$ equiv.) was added potionwise. After 30 min ethyl bromide $(5.55 \mathrm{~g}$, $50.9 \mathrm{mmol}, 2.6$ equiv.) was added dropwise. After $22 \mathrm{~h}$ reaction was quenched with $\mathrm{NH}_{4} \mathrm{Cl}_{\mathrm{aq}}(50 \mathrm{~mL}$, $10 \% \mathrm{w} / \mathrm{v})$. Resulted mixture was extracted with ethyl acetate $(3 \times 50 \mathrm{~mL})$, combined organic phases were washed with $\mathrm{H}_{2} \mathrm{O}(50 \mathrm{~mL})$, brine $(50 \mathrm{~mL})$, and dried over anhydrous $\mathrm{MgSO}_{4}$. Mixture was filtered and evaporated. Crude ethyl phenyl selenide was placed in $1 \mathrm{~L}$ round-bottom flask, dichloromethane (350 mL), and $\mathrm{K}_{2} \mathrm{CO}_{3}$ aq $(250 \mathrm{~mL}$, sat.) were added, and to mechanically stirred mixture $\mathrm{mCPBA}(26.9 \mathrm{~g}$, $120.0 \mathrm{mmol}$, ca. $77 \%$ purity, 6.0 equiv.) was added portionwise. After $1.5 \mathrm{~h} \mathrm{H}_{2} \mathrm{O}(300 \mathrm{~mL}$ ) was added, phases were separated, aqueous phase was extracted with dichloromethane $(2 \times 100 \mathrm{~mL})$, combined organic phases were washed with $\mathrm{H}_{2} \mathrm{O}(200 \mathrm{~mL})$, brine $(200 \mathrm{~mL})$, and dried over anhydrous $\mathrm{MgSO}_{4}$. Mixture was filtered and evaporated yielding $\mathbf{2 b}$ ( $6.67 \mathrm{~g}, 30.7 \mathrm{mmol}, \mathbf{7 7 \%}$ over $\mathbf{2}$ steps) as a white solid.

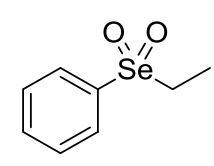

ethyl phenyl selenone, $\mathbf{2 b}$

white solid, $\mathrm{mp} 76.0-78.0^{\circ} \mathrm{C}$

$R_{f}=0.52$ (dichloromethane/methanol 9:1)

${ }^{1} \mathbf{H}$ NMR $\left(400 \mathrm{MHz} \mathrm{CDCl}_{3}\right): \delta=7.89-7.85(\mathrm{~m}, 2 \mathrm{H}), 7.66-7.60(\mathrm{~m}, 1 \mathrm{H})$, $7.59-7.53(\mathrm{~m}, 2 \mathrm{H}), 3.46(\mathrm{q}, J=7.6 \mathrm{~Hz}, 2 \mathrm{H}), 1.43(\mathrm{t}, J=7.6 \mathrm{~Hz}, 3 \mathrm{H})$.

${ }^{13} \mathrm{C} \mathrm{NMR}\left(100 \mathrm{MHz}, \mathrm{CDCl}_{3}\right): \delta=140.0,134.2,130.1,127.0,54.4,7.1$.

HRMS (ESI): $\mathrm{m} / \mathrm{z}[\mathrm{M}+\mathrm{Na}]^{+}$calcd for $\mathrm{C}_{8} \mathrm{H}_{10} \mathrm{O}_{2} \mathrm{SeNa}$ : 240.9744; found: 240.9740 


\section{Synthesis of $n$-butyl phenyl selenone (2c)}

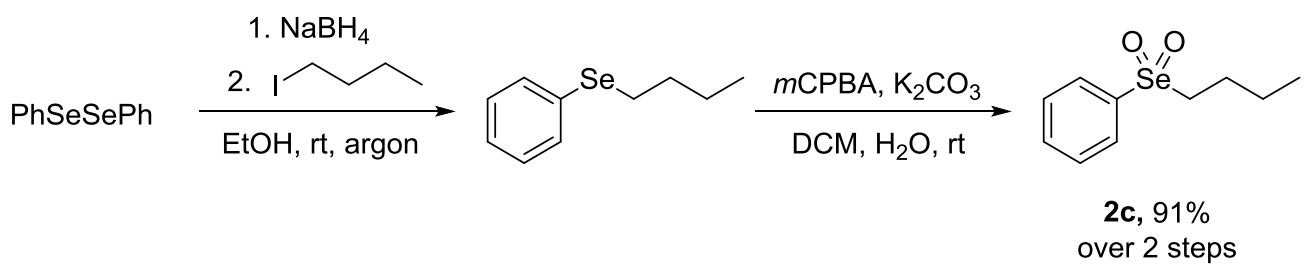

A $100 \mathrm{~mL}$ round-bottom flask was charged with diphenyl diselenide $(2.50 \mathrm{~g}, 8.01 \mathrm{mmol}, 1.0$ equiv.), flushed with argon and immersed in water bath (rt). Absolute ethanol $(21 \mathrm{~mL})$ was added and $\mathrm{NaBH}_{4}$ (1.32 g, $34.9 \mathrm{mmol}, 4.4$ equiv.) was added potionwise. After $1 \mathrm{~h}$ 1-butyl iodide $(4.16 \mathrm{~g}, 22.6 \mathrm{mmol}$, 2.8 equiv.) was added dropwise. After $20 \mathrm{~h}$ reaction was quenched with $\mathrm{NH}_{4} \mathrm{Cl}_{\text {aq }}(50 \mathrm{~mL}, 10 \% \mathrm{w} / \mathrm{v})$. Resulted mixture was extracted with ethyl acetate $(3 \times 50 \mathrm{~mL})$, combined organic phases were washed with $\mathrm{H}_{2} \mathrm{O}(50 \mathrm{~mL})$, brine $(50 \mathrm{~mL})$, and dried over anhydrous $\mathrm{MgSO}_{4}$. Mixture was filtered and evaporated. Crude n-butyl phenyl selenide was placed in $500 \mathrm{~mL}$ round-bottom flask, dichloromethane $(110 \mathrm{~mL})$, and $\mathrm{K}_{2} \mathrm{CO}_{3}$ aq $(110 \mathrm{~mL}$, sat.) were added, and to mechanically stirred mixture $m$ CPBA ( $26.9 \mathrm{~g}, 120.0 \mathrm{mmol}$, ca. $77 \%$ purity, 15.0 equiv.) was added portionwise. After $2.0 \mathrm{~h}$ $\mathrm{H}_{2} \mathrm{O}$ (150 mL) was added, phases were separated, aqueous phase was extracted with dichloromethane $(2 \times 150 \mathrm{~mL})$, combined organic phases were washed with $\mathrm{Na}_{2} \mathrm{~S}_{2} \mathrm{O}_{3}$ aq $(150 \mathrm{~mL}, 10 \%$ $\mathrm{w} / \mathrm{v}), \mathrm{H}_{2} \mathrm{O}(150 \mathrm{~mL})$, brine $(150 \mathrm{~mL})$, and dried over anhydrous $\mathrm{MgSO}_{4}$. Mixture was filtered, evaporated, and separated with column chromatography (eluent: dichloromethane/ethyl acetate $3: 1$ ) yielding $2 \mathrm{c}$ ( $3.59 \mathrm{~g}, 14.6 \mathrm{mmol}, \mathbf{9 1 \%}$ over 2 steps) as a white solid.

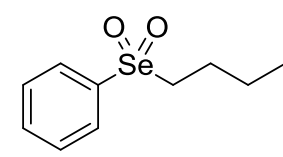

n-butyl phenyl selenone, $\mathbf{2 c}$

white solid, $\mathrm{mp} 43.0-45.5^{\circ} \mathrm{C}$

eluent: dichloromethane/ethyl acetate $3: 1$

$\mathrm{R}_{\mathrm{f}}=0.61$ (dichloromethane/methanol 9:1)

${ }^{1} \mathrm{H}$ NMR (400 MHz, $\left.\mathrm{CDCl}_{3}\right): \delta=7.86-7.76(\mathrm{~m}, 2 \mathrm{H}), 7.59-7.53(\mathrm{~m}, 1 \mathrm{H})$, $7.51-7.46(\mathrm{~m}, 2 \mathrm{H}), 3.37(\mathrm{t}, J=8.1 \mathrm{~Hz}, 2 \mathrm{H}), 1.80-1.67(\mathrm{~m}, 2 \mathrm{H}), 1.32(\mathrm{~h}$, $J=7.4 \mathrm{~Hz}, 2 \mathrm{H}), 0.75(\mathrm{t}, J=7.4 \mathrm{~Hz}, 3 \mathrm{H})$.

${ }^{13} \mathrm{C}$ NMR (100 MHz, $\left.\mathrm{CDCl}_{3}\right): \delta=140.7,133.9,129.9,126.6,59.3,23.9$, 21.4, 13.0.

${ }^{1} \mathrm{H}$ NMR, ${ }^{13} \mathrm{C}$ NMR spectra were consistent with those reported in the literature. ${ }^{[3]}$ 


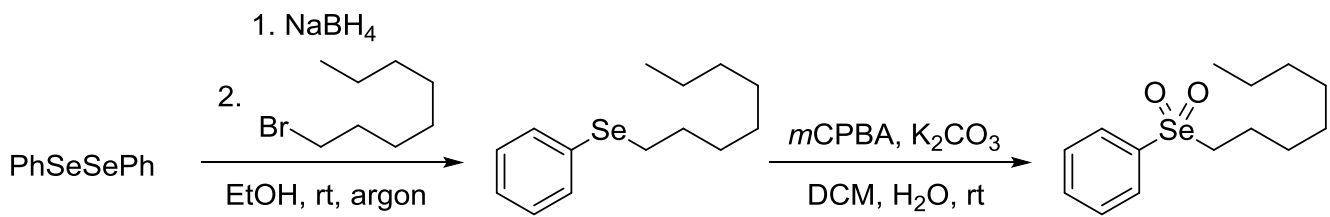

2d, $89 \%$

over 2 steps

A $100 \mathrm{~mL}$ round-bottom flask was charged with diphenyl diselenide $(4.68 \mathrm{~g}, 15.0 \mathrm{mmol}, 1.0$ equiv.), flushed with argon and immersed in water bath (rt). Absolute ethanol $(40 \mathrm{~mL})$ was added and $\mathrm{NaBH}_{4}$ ( $2.32 \mathrm{~g}, 61.4 \mathrm{mmol}, 4.1$ equiv.) was added potionwise. After $1.5 \mathrm{~h}$ 1-bromooctane $(7.24 \mathrm{~g}, 37.5 \mathrm{mmol}$, 2.5 equiv.) was added dropwise. After $23 \mathrm{~h}$ reaction was quenched with $\mathrm{NH}_{4} \mathrm{Cl}_{\text {aq }}(50 \mathrm{~mL}, 10 \% \mathrm{w} / \mathrm{v})$. Resulted mixture was extracted with ethyl acetate $(3 \times 50 \mathrm{~mL})$, combined organic phases were washed with $\mathrm{H}_{2} \mathrm{O}(50 \mathrm{~mL})$, brine $(50 \mathrm{~mL})$, and dried over anhydrous $\mathrm{MgSO}_{4}$. Mixture was filtered and evaporated. Crude $n$-octyl phenyl selenide was placed in $1 \mathrm{~L}$ round-bottom flask, dichloromethane $(200 \mathrm{~mL})$, and $\mathrm{K}_{2} \mathrm{CO}_{3}$ aq $(200 \mathrm{~mL}$, sat.) were added, and to mechanically stirred mixture $\mathrm{mCPBA}$ (20.3 g, $90.4 \mathrm{mmol}$, ca. $77 \%$ purity, 6.0 equiv.) was added portionwise. After $4.5 \mathrm{~h} \mathrm{H}_{2} \mathrm{O}(200 \mathrm{~mL})$ was added, phases were separated, aqueous phase was extracted with dichloromethane $(2 \times 200 \mathrm{~mL})$, combined organic phases were washed with $\mathrm{Na}_{2} \mathrm{~S}_{2} \mathrm{O}_{3}$ aq $(200 \mathrm{~mL}, 10 \% \mathrm{w} / \mathrm{v}), \mathrm{H}_{2} \mathrm{O}(200 \mathrm{~mL})$, brine $(200 \mathrm{~mL})$, and dried over anhydrous $\mathrm{MgSO}_{4}$. Mixture was filtered, evaporated, and separated with column chromatography (eluent: dichloromethane to dichloromethane/ethyl acetate 1:1) yielding $\mathbf{2 d}(8.08 \mathrm{~g}$, $26.8 \mathrm{mmol}, \mathbf{8 9 \%}$ over 2 steps) as a white solid.<smiles>CCCCCCCS(=O)(=O)c1ccccc1</smiles>

n-octyl phenyl selenone, $\mathbf{2 d}$

white solid, $\mathrm{mp} 26.0-27.5^{\circ} \mathrm{C}$

eluent: dichloromethane to dichloromethane/ethyl acetate 1:1

$R_{f}=0.69$ (dichloromethane/methanol 9:1)

${ }^{1} \mathrm{H}$ NMR (400 MHz, $\left.\mathrm{CDCl}_{3}\right): \delta=\delta 7.97-7.91(\mathrm{~m}, 2 \mathrm{H}), 7.72-7.66(\mathrm{~m}, 1 \mathrm{H})$, $7.65-7.58(\mathrm{~m}, 2 \mathrm{H}), 3.44(\mathrm{t}, J=8.0 \mathrm{~Hz}, 2 \mathrm{H}), 1.91(\mathrm{p}, J=7.9 \mathrm{~Hz}, 2 \mathrm{H}), 1.47-$ $1.35(\mathrm{~m}, 2 \mathrm{H}), 1.29-1.12(\mathrm{~m}, 8 \mathrm{H}), 0.83(\mathrm{t}, J=6.9 \mathrm{~Hz}, 3 \mathrm{H})$.

${ }^{13} \mathrm{C}$ NMR (100 MHz, $\left.\mathrm{CDCl}_{3}\right): \delta=141.2,134.2,130.2,127.1,60.0,31.6$, 28.78, 28.76, 28.5, 22.5, 22.4, 14.0 .

HRMS (ESI): $\mathrm{m} / \mathrm{z}$ [M+Na] ${ }^{+}$calcd for $\mathrm{C}_{14} \mathrm{H}_{22} \mathrm{O}_{2} \mathrm{SeNa}$ : 325.0683; found: 325.0680 


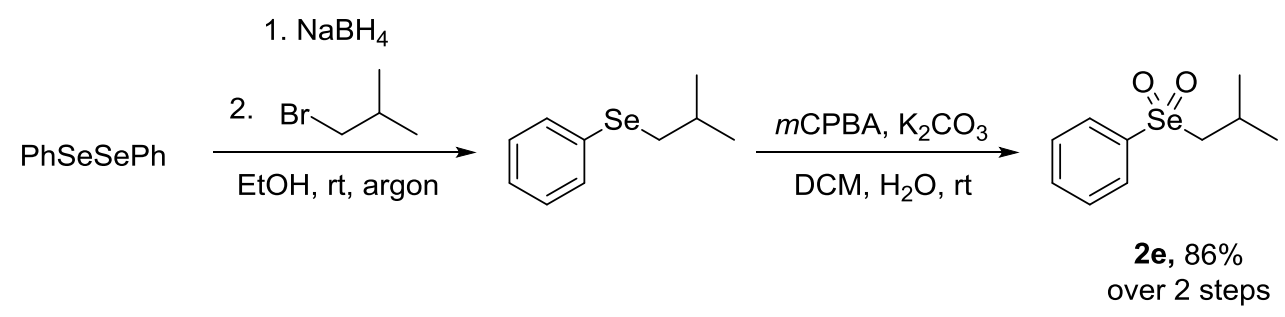

A $100 \mathrm{~mL}$ round-bottom flask was charged with diphenyl diselenide $(5.00 \mathrm{~g}, 16.0 \mathrm{mmol}, 1.0$ equiv.), flushed with argon and immersed in water bath (rt). Absolute ethanol $(40 \mathrm{~mL})$ was added and $\mathrm{NaBH}_{4}$ (1.81 g, $47.8 \mathrm{mmol}, 3.0$ equiv.) was added potionwise. After $45 \mathrm{~min}$ 1-bromo-2-methylpropane ( $5.49 \mathrm{~g}, 40.0 \mathrm{mmol}, 2.5$ equiv.) was added dropwise. After $20 \mathrm{~h}$ reaction was quenched with $\mathrm{NH}_{4} \mathrm{Cl}_{\mathrm{aq}}$ $(50 \mathrm{~mL}, 10 \% \mathrm{w} / \mathrm{v})$. Resulted mixture was extracted with ethyl acetate $(3 \times 50 \mathrm{~mL})$, combined organic phases were washed with $\mathrm{H}_{2} \mathrm{O}(50 \mathrm{~mL})$, brine $(50 \mathrm{~mL})$, and dried over anhydrous $\mathrm{MgSO}_{4}$. Mixture was filtered and evaporated. Crude isobutyl phenyl selenide was placed in $1 \mathrm{~L}$ round-bottom flask, dichloromethane $(200 \mathrm{~mL})$, and $\mathrm{K}_{2} \mathrm{CO}_{3}$ aq $(200 \mathrm{~mL}$, sat.) were added, and to mechanically stirred mixture $m$ CPBA ( $21.6 \mathrm{~g}, 96.2 \mathrm{mmol}$, ca. $77 \%$ purity, 6.0 equiv.) was added portionwise. After $5 \mathrm{~h} \mathrm{H}_{2} \mathrm{O}$ $(200 \mathrm{~mL}$ ) was added, phases were separated, aqueous phase was extracted with dichloromethane (2 $x 200 \mathrm{~mL})$, combined organic phases were washed with $\mathrm{Na}_{2} \mathrm{~S}_{2} \mathrm{O}_{3}$ aq $(200 \mathrm{~mL}, 10 \% \mathrm{w} / \mathrm{v}), \mathrm{H}_{2} \mathrm{O}(200 \mathrm{~mL})$, brine $(200 \mathrm{~mL})$, and dried over anhydrous $\mathrm{MgSO}_{4}$. Mixture was filtered, evaporated, and separated with column chromatography (eluent: dichloromethane to dichloromethane/ethyl acetate 3:1) yielding $2 \mathrm{e}(6.75 \mathrm{~g}, 27.5 \mathrm{mmol}, \mathbf{8 6} \%$ over 2 steps) as a white crystals.

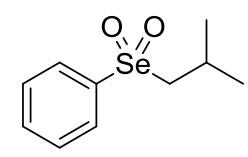

isobutyl phenyl selenone, $\mathbf{2 e}$

white crystals, $\mathrm{mp} 58.5-60.5^{\circ} \mathrm{C}$

eluent: dichloromethane to dichloromethane/ethyl acetate 3:1

$\mathrm{R}_{\mathrm{f}}=0.61$ (dichloromethane/methanol 9:1)

${ }^{1} \mathbf{H}$ NMR (400 MHz, $\left.\mathrm{CDCl}_{3}\right): \delta=7.95-7.87(\mathrm{~m}, 2 \mathrm{H}), 7.67-7.62(\mathrm{~m}, 1 \mathrm{H})$, $7.60-7.55(\mathrm{~m}, 2 \mathrm{H}), 3.35(\mathrm{~d}, J=6.9 \mathrm{~Hz}, 2 \mathrm{H}), 2.53-2.31(\mathrm{~m}, 1 \mathrm{H}), 1.08(\mathrm{~d}, J$ $=6.7 \mathrm{~Hz}, 6 \mathrm{H})$.

${ }^{13} \mathrm{C}$ NMR (100 MHz, $\left.\mathrm{CDCl}_{3}\right): \delta=142.2,134.0,130.1,126.7,68.0,24.4$, 22.3.

HRMS (ESI): $\mathrm{m} / \mathrm{z}$ [M+Na] ${ }^{+}$calcd for $\mathrm{C}_{10} \mathrm{H}_{14} \mathrm{O}_{2} \mathrm{SeNa}$ : 269.0057; found: 269.0049 


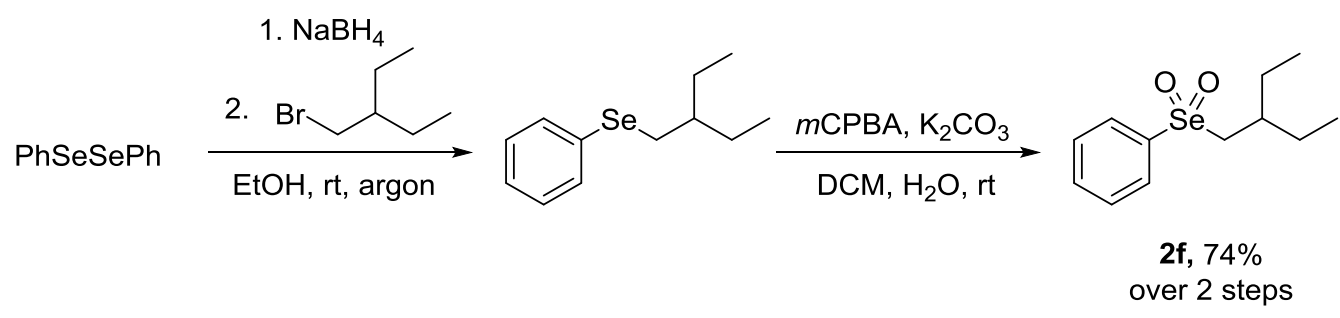

A $100 \mathrm{~mL}$ round-bottom flask was charged with diphenyl diselenide $(4.87 \mathrm{~g}, 15.6 \mathrm{mmol}, 1.0$ equiv.), flushed with argon and immersed in water bath (rt). Absolute ethanol $(40 \mathrm{~mL})$ was added and $\mathrm{NaBH}_{4}$ (1.83 g, $48.4 \mathrm{mmol}, 3.1$ equiv.) was added potionwise. After $30 \mathrm{~min}$ 1-bromo-2-ethylbutane $(5.90 \mathrm{~g}$, $35.7 \mathrm{mmol}, 2.3$ equiv.) was added dropwise. After $18 \mathrm{~h}$ reaction was quenched with $\mathrm{NH}_{4} \mathrm{Cl}_{\text {aq }}(50 \mathrm{~mL}$, $10 \% \mathrm{w} / \mathrm{v})$. Resulted mixture was extracted with ethyl acetate $(3 \times 50 \mathrm{~mL})$, combined organic phases were washed with $\mathrm{H}_{2} \mathrm{O}(50 \mathrm{~mL})$, brine $(50 \mathrm{~mL})$, and dried over anhydrous $\mathrm{MgSO}_{4}$. Mixture was filtered and evaporated. Crude 2-ethylbutyl phenyl selenide was placed in $1 \mathrm{~L}$ round-bottom flask, dichloromethane $(200 \mathrm{~mL})$, and $\mathrm{K}_{2} \mathrm{CO}_{3}$ aq $(200 \mathrm{~mL}$, sat.) were added, and to mechanically stirred mixture $m$ CPBA ( $21.1 \mathrm{~g}, 94.1 \mathrm{mmol}$, ca. $77 \%$ purity, 6.0 equiv.) was added portionwise. After $6 \mathrm{~h} \mathrm{H}_{2} \mathrm{O}$ $(300 \mathrm{~mL}$ ) was added, phases were separated, aqueous phase was extracted with dichloromethane (2 $x 200 \mathrm{~mL})$, combined organic phases were washed with $\mathrm{H}_{2} \mathrm{O}(200 \mathrm{~mL})$, brine $(200 \mathrm{~mL})$, and dried over anhydrous $\mathrm{MgSO}_{4}$. Mixture was filtered, evaporated and separated with column chromatography (eluent: dichloromethane to dichloromethane/ethyl acetate 3:1) yielding $\mathbf{2 f}(6.27 \mathrm{~g}, 23.0 \mathrm{mmol}, \mathbf{7 4 \%}$ over 2 steps) as white crystals.<smiles>CCC(CC)CS(=O)(=O)c1ccccc1</smiles>

2-ethylbutyl phenyl selenone, $\mathbf{2 f}$

white crystals, $\mathrm{mp} 60.0-63.0^{\circ} \mathrm{C}$

eluent: dichloromethane to dichloromethane/ethyl acetate 3:1

$\mathrm{R}_{\mathrm{f}}=0.68$ (dichloromethane/methanol 9:1)

${ }^{1} \mathbf{H}$ NMR (400 MHz, $\left.\mathrm{CDCl}_{3}\right): \delta=7.99-7.91(\mathrm{~m}, 2 \mathrm{H}), 7.70-7.65(\mathrm{~m}, 1 \mathrm{H})$, $7.64-7.57(\mathrm{~m}, 2 \mathrm{H}), 3.42(\mathrm{~d}, J=6.6 \mathrm{~Hz}, 2 \mathrm{H}), 2.18-2.03(\mathrm{~m}, 1 \mathrm{H}), 1.62-$ $1.41(\mathrm{~m}, 4 \mathrm{H}), 0.83(\mathrm{t}, J=7.5 \mathrm{~Hz}, 6 \mathrm{H})$.

${ }^{13} \mathrm{C}$ NMR (100 MHz, $\left.\mathrm{CDCl}_{3}\right): \delta=142.4,134.1,130.2,126.8,64.5,36.0$, $25.1,10.2$.

HRMS (ESI): $\mathrm{m} / \mathrm{z}[\mathrm{M}+\mathrm{Na}]^{+}$calcd for $\mathrm{C}_{12} \mathrm{H}_{18} \mathrm{O}_{2} \mathrm{SeNa}$ : 297.0370; found: 297.0361 


\section{Synthesis of neopentyl phenyl selenone $(2 \mathrm{~g})$}

Alkylating agent used for synthesis of neopentyl phenyl selenone (2g) was neopentyl methanesulfonate, which was synthesised according to the literature. ${ }^{[4]}$

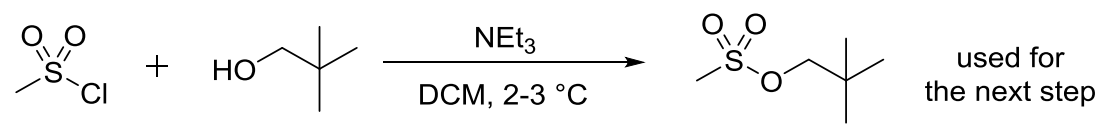

A $250 \mathrm{~mL}$ round-bottom flask was charged with neopentyl alcohol (4.43 g, $50.3 \mathrm{mmol}, 1.0$ equiv.), dichloromethane ( $60 \mathrm{~mL}$ ), triethylamine $(5.8 \mathrm{~g}, 57.0 \mathrm{mmol}, 1.1$ equiv.), flushed with argon and cooled in ice/water bath (ca. 2-3 ${ }^{\circ} \mathrm{C}$ ). Methanesulfonyl chloride $(6.49 \mathrm{~g}, 56.7 \mathrm{mmol}, 1.1$ equiv.) was added dropwise. After $2.5 \mathrm{~h} \mathrm{H}_{2} \mathrm{O}(50 \mathrm{~mL})$ was added, phases were separated, aqueous phase was extracted with ethyl acetate $(3 \times 50 \mathrm{~mL})$, combined organic phases were washed with $\mathrm{H}_{2} \mathrm{O}(50 \mathrm{~mL})$, brine (50 $\mathrm{mL}$ ), and dried over anhydrous $\mathrm{MgSO}_{4}$. Mixture was filtered, evaporated, and crude product was separated with distillation $\left(78^{\circ} \mathrm{C}, 4 \mathrm{mbar}\right)$ yielding neopentyl methanesulfonate $(6.17 \mathrm{~g}, 37.1 \mathrm{mmol}$, $\mathbf{7 4 \% )}$ as a colorless oil.

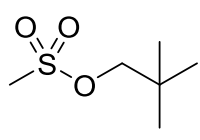

neopentyl methanesulfonate colorless oil

${ }^{1} \mathrm{H}$ NMR $\left(400 \mathrm{MHz}, \mathrm{CDCl}_{3}\right): \delta=3.77(\mathrm{~s}, 2 \mathrm{H}), 2.91(\mathrm{~s}, 3 \mathrm{H}), 0.89(\mathrm{~s}, 9 \mathrm{H})$.

${ }^{13} \mathrm{C} \mathrm{NMR}\left(100 \mathrm{MHz}, \mathrm{CDCl}_{3}\right): \delta=78.9,36.6,31.4,25.7$.

${ }^{1} \mathrm{H} N M R,{ }^{13} \mathrm{C}$ NMR spectra and were consistent with those reported in the literature. ${ }^{[4]}$
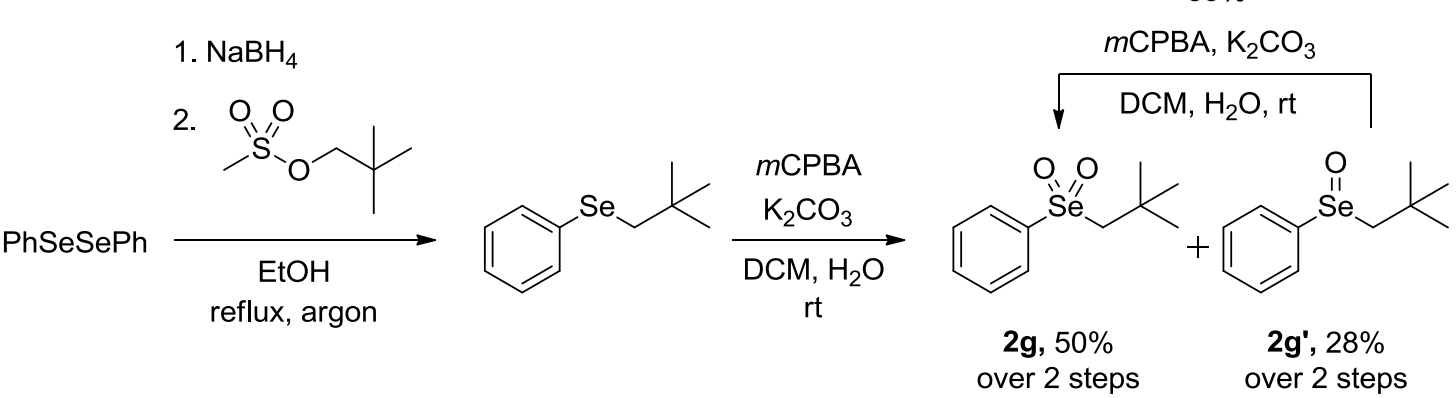

A $100 \mathrm{~mL}$ round-bottom flask was charged with diphenyl diselenide $(5.00 \mathrm{~g}, 16.0 \mathrm{mmol}, 1.0$ equiv.), flushed with argon and immersed in water bath $(\mathrm{rt})$. Absolute ethanol $(40 \mathrm{~mL})$ was added and $\mathrm{NaBH}_{4}$ $(1.83 \mathrm{~g}, 48.4 \mathrm{mmol}, 3.0$ equiv.) was added potionwise. After $70 \mathrm{~min}$ neopentyl methanesulfonate 
(6.64 g, $39.9 \mathrm{mmol}, 2.5$ equiv.) was added dropwise. After $20 \mathrm{~h}$ reaction was refluxed for $20 \mathrm{~h}$, and was quenched with $\mathrm{NH}_{4} \mathrm{Cl}_{a q}(50 \mathrm{~mL}, 10 \% \mathrm{w} / \mathrm{v})$. Resulted mixture was extracted with ethyl acetate $(3 \times 50 \mathrm{~mL})$, combined organic phases were washed with $\mathrm{H}_{2} \mathrm{O}(50 \mathrm{~mL})$, brine $(50 \mathrm{~mL})$, and dried over anhydrous $\mathrm{MgSO}_{4}$. Mixture was filtered, evaporated, and separated with column chromatography (eluent: cyclohexane to cyclohexane/ethyl acetate 10:1). Crude neopentyl phenyl selenide was placed in $1 \mathrm{~L}$ round-bottom flask, dichloromethane $(200 \mathrm{~mL})$, and $\mathrm{K}_{2} \mathrm{CO}_{3}$ aq $(200 \mathrm{~mL}$, sat.) were added, and to mechanically stirred mixture $m$ CPBA $(21.5 \mathrm{~g}, 96.1 \mathrm{mmol}, \mathrm{ca} .77 \%$ purity, 6.0 equiv.) was added portionwise. After $6 \mathrm{~h} \mathrm{H}_{2} \mathrm{O}(200 \mathrm{~mL})$ was added, phases were separated, aqueous phase was extracted with dichloromethane $(2 \times 200 \mathrm{~mL})$, combined organic phases were washed with $\mathrm{H}_{2} \mathrm{O}(200$ $\mathrm{mL})$, brine $(200 \mathrm{~mL})$, and dried over anhydrous $\mathrm{MgSO}_{4}$. Mixture was filtered, evaporated, and separated with column chromatography (eluent: dichloromethane/ethyl acetate $3: 1$, then dichloromethane/methanol 9:1) yielding $\mathbf{2 g}(4.17 \mathrm{~g}, 16.1 \mathrm{mmol}, \mathbf{5 0 \%}$ over 2 steps) as white crystals and $\mathbf{2} \mathbf{g}^{\prime}(2.21 \mathrm{~g}, 9.10 \mathrm{mmol}, \mathbf{2 8} \%$ over $\mathbf{2}$ steps $)$ as white crystals.

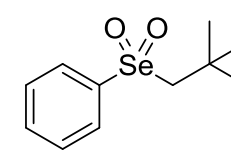

neopentyl phenyl selenone, $\mathbf{2 g}$

white crystals, $\mathrm{mp} 92.0-94.5^{\circ} \mathrm{C}$

eluent: dichloromethane/ethyl acetate $3: 1$ then

dichloromethane/methanol 9:1

$R_{f}=0.66$ (dichloromethane/methanol 9:1)

${ }^{1} \mathrm{H}$ NMR (400 MHz, $\left.\mathrm{CDCl}_{3}\right): \delta=7.91-7.87(\mathrm{~m}, 2 \mathrm{H}), 7.63-7.57(\mathrm{~m}, 1 \mathrm{H})$, $7.56-7.50(\mathrm{~m}, 2 \mathrm{H}), 3.41(\mathrm{~s}, 2 \mathrm{H}), 1.17(\mathrm{~s}, 9 \mathrm{H})$.

${ }^{13} \mathrm{C}$ NMR $\left(100 \mathrm{MHz}, \mathrm{CDCl}_{3}\right): \delta=143.8,133.8,130.0,126.4,73.5,32.9$, 29.4.

HRMS (ESI): $\mathrm{m} / \mathrm{z}[\mathrm{M}+\mathrm{Na}]^{+}$calcd for $\mathrm{C}_{11} \mathrm{H}_{16} \mathrm{O}_{2} \mathrm{SeNa}: 283.0213$; found: 283.0205

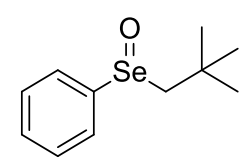

neopentyl phenyl selenoxide, $\mathbf{2} \mathbf{g}^{\prime}$

white crystals, $\mathrm{mp} 54.5-56.5^{\circ} \mathrm{C}$

eluent: dichloromethane/ethyl acetate 3:1 then

dichloromethane/methanol 9:1

$R_{f}=0.46$ (dichloromethane/methanol 9:1)

${ }^{1}$ H NMR (400 MHz, $\left.\mathrm{CDCl}_{3}\right): \delta=7.72-7.62(\mathrm{~m}, 2 \mathrm{H}), 7.55-7.37(\mathrm{~m}, 3 \mathrm{H})$, $2.91(\mathrm{~d}, J=12.3 \mathrm{~Hz}, 1 \mathrm{H}), 2.80(\mathrm{~d}, J=12.3 \mathrm{~Hz}, 1 \mathrm{H}), 1.13(\mathrm{~s}, 9 \mathrm{H})$.

${ }^{13} \mathrm{C}$ NMR $\left(100 \mathrm{MHz}, \mathrm{CDCl}_{3}\right): \delta=141.5,131.0,129.5,125.8,72.3,31.9$, 30.1.

HRMS (ESI): $\mathrm{m} / \mathrm{z}[\mathrm{M}+\mathrm{H}]^{+}$calcd for $\mathrm{C}_{11} \mathrm{H}_{17} \mathrm{OSe}$ : 245.0445; found: 245.0440 


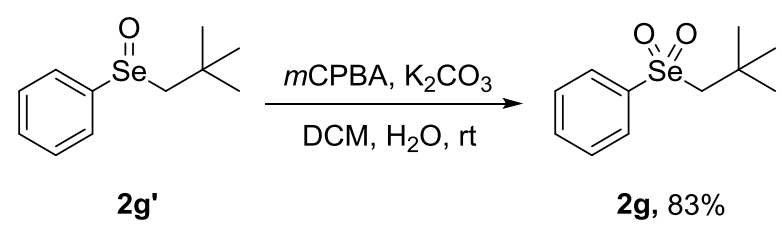

A $500 \mathrm{~mL}$ round-bottom flask was charged with neopentyl phenyl selenoxide $(1.88 \mathrm{~g}, 7.75 \mathrm{mmol}$, 1.0 equiv.), dichloromethane $(50 \mathrm{~mL}), \mathrm{K}_{2} \mathrm{CO}_{3}$ aq $(50 \mathrm{~mL}$, sat.), and to mechanically stirred mixture $m$ CPBA (5.24 g, 23.4 mmol, ca. 77\% purity, 3.0 equiv.) was added portionwise. After $16 \mathrm{~h} \mathrm{H}_{2} \mathrm{O}$ (100 $\mathrm{mL})$ was added, phases were separated, aqueous phase was extracted with dichloromethane $(2 \times 100$ $\mathrm{mL})$, combined organic phases were washed with $\mathrm{Na}_{2} \mathrm{~S}_{2} \mathrm{O}_{3}$ aq $(100 \mathrm{~mL}, 10 \% \mathrm{w} / \mathrm{v}), \mathrm{H}_{2} \mathrm{O}(100 \mathrm{~mL})$, brine $(100 \mathrm{~mL})$, and dried over anhydrous $\mathrm{MgSO}_{4}$. Mixture was filtered, evaporated, and separated with column chromatography (eluent: dichloromethane to dichloromethane/ethyl acetate 3:1) yielding $\mathbf{2 g}$ $(1.66 \mathrm{~g}, 6.40 \mathrm{mmol}, 83 \%)$ as white crystals. 


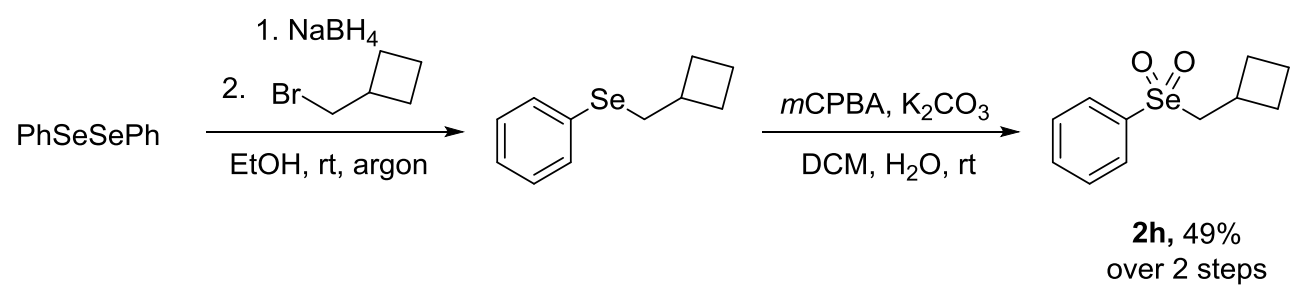

A $100 \mathrm{~mL}$ round-bottom flask was charged with diphenyl diselenide (4.81 g, $15.4 \mathrm{mmol}, 1.0$ equiv.), flushed with argon and immersed in water bath $(\mathrm{rt})$. Absolute ethanol $(40 \mathrm{~mL})$ was added and $\mathrm{NaBH}_{4}$ $(1.90 \mathrm{~g}, 50.1 \mathrm{mmol}, 3.3$ equiv.) was added potionwise. After $30 \mathrm{~min}$ bromomethylcyclobutane $(4.91 \mathrm{~g}$, $32.9 \mathrm{mmol}, 2.1$ equiv.) was added dropwise. After $20 \mathrm{~h}$ reaction was quenched with $\mathrm{NH}_{4} \mathrm{Cl}_{\mathrm{aq}}(50 \mathrm{~mL}$, $10 \% \mathrm{w} / \mathrm{v})$. Resulted mixture was extracted with ethyl acetate $(3 \times 50 \mathrm{~mL})$, combined organic phases were washed with $\mathrm{H}_{2} \mathrm{O}(50 \mathrm{~mL})$, brine $(50 \mathrm{~mL})$, and dried over anhydrous $\mathrm{MgSO}_{4}$. Mixture was filtered and evaporated. Crude cyclobutylmethyl phenyl selenide was placed in $1 \mathrm{~L}$ round-bottom flask, dichloromethane $(200 \mathrm{~mL})$, and $\mathrm{K}_{2} \mathrm{CO}_{3}$ aq $(200 \mathrm{~mL}$, sat.) were added, and to mechanically stirred mixture $m C P B A\left(21.4 \mathrm{~g}, 95.7 \mathrm{mmol}\right.$, ca $77 \%$ purity, 6.2 equiv.) was added portionwise. After $6 \mathrm{~h} \mathrm{H}_{2} \mathrm{O}$ $(200 \mathrm{~mL}$ ) was added, phases were separated, aqueous phase was extracted with dichloromethane (2 $\times 200 \mathrm{~mL})$, combined organic phases were washed with $\mathrm{H}_{2} \mathrm{O}(200 \mathrm{~mL})$, brine $(200 \mathrm{~mL})$, and dried over anhydrous $\mathrm{MgSO}_{4}$. Mixture was filtered, evaporated, and separated with column chromatography (eluent: dichloromethane to dichloromethane/ethyl acetate 3:1) yielding $2 \mathrm{~h}$ (3.69 g, $15.1 \mathrm{mmol}, \mathbf{4 9 \%}$ over 2 steps) as a white solid.

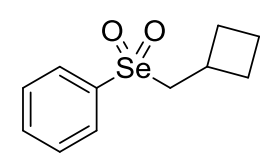

cyclobutylmethyl phenyl selenone, $\mathbf{2 h}$

white solid, $\mathrm{mp} 80.0^{\circ} \mathrm{C}$ (dec.)

eluent: dichloromethane to dichloromethane/ethyl acetate 3:1

$\mathrm{Rf}=0.64$ (dichloromethane/methanol 9:1)

${ }^{1} \mathbf{H}$ NMR (400 MHz, $\left.\mathrm{CDCl}_{3}\right): \delta=7.96-7.89(\mathrm{~m}, 2 \mathrm{H}), 7.70-7.64(\mathrm{~m}, 1 \mathrm{H})$, $7.63-7.55(\mathrm{~m}, 2 \mathrm{H}), 3.58(\mathrm{~d}, J=7.7 \mathrm{~Hz}, 2 \mathrm{H}), 2.99-2.80(\mathrm{~m}, 1 \mathrm{H}), 2.15-$ $2.06(\mathrm{~m}, 2 \mathrm{H}), 1.96-1.71(\mathrm{~m}, 4 \mathrm{H})$.

${ }^{13} \mathrm{C}$ NMR (100 MHz, $\left.\mathrm{CDCl}_{3}\right): \delta=141.8,134.1,130.1,127.0,65.7,29.4$, 28.5, 18.7 .

HRMS (ESI): $\mathrm{m} / \mathrm{z}[\mathrm{M}+\mathrm{Na}]^{+}$calcd for $\mathrm{C}_{11} \mathrm{H}_{14} \mathrm{O}_{2} \mathrm{SeNa}$ : 281.0057; found: 281.0048 


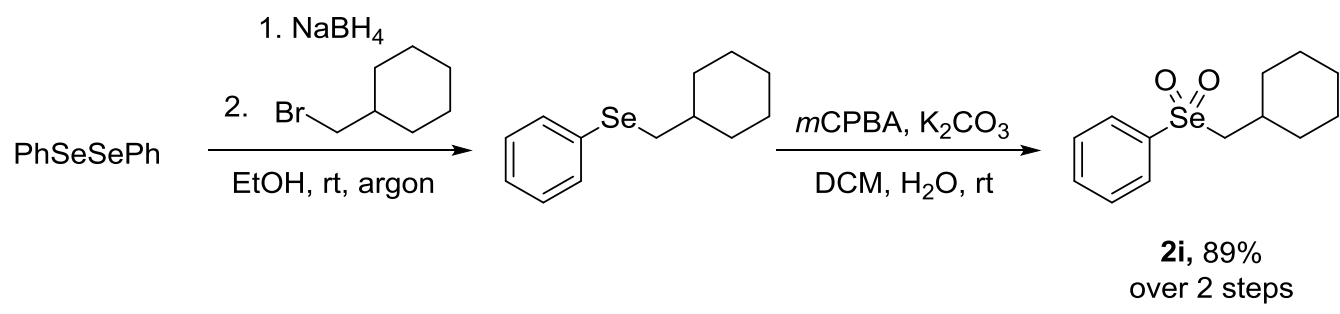

A $100 \mathrm{~mL}$ round-bottom flask was charged with diphenyl diselenide (5.39 g, $17.3 \mathrm{mmol}, 1.0$ equiv.), flushed with argon and immersed in water bath (rt). Absolute ethanol $(45 \mathrm{~mL})$ was added and $\mathrm{NaBH}_{4}$ ( $1.97 \mathrm{~g}, 52.0 \mathrm{mmol}, 3.0$ equiv.) was added potionwise. After $45 \mathrm{~min}$ bromomethylcyclohexane (7.66 $\mathrm{g}, 43.4 \mathrm{mmol}, 2.5$ equiv.) was added dropwise. After $24 \mathrm{~h}$ reaction was quenched with $\mathrm{NH}_{4} \mathrm{Cl}_{\mathrm{aq}}(50$ $\mathrm{mL}, 10 \% \mathrm{w} / \mathrm{v})$. Resulted mixture was extracted with ethyl acetate $(3 \times 50 \mathrm{~mL})$, combined organic phases were washed with $\mathrm{H}_{2} \mathrm{O}(50 \mathrm{~mL})$, brine $(50 \mathrm{~mL})$, and dried over anhydrous $\mathrm{MgSO}_{4}$. Mixture was filtered and evaporated. Crude cyclohexylmethyl phenyl selenide was placed in $1 \mathrm{~L}$ round-bottom flask, dichloromethane $(200 \mathrm{~mL})$, and $\mathrm{K}_{2} \mathrm{CO}_{3}$ aq $(200 \mathrm{~mL}$, sat.) were added, and to mechanically stirred mixture $m$ CPBA ( $24.4 \mathrm{~g}, 108.3 \mathrm{mmol}, 77 \%$ purity, 6.3 equiv.) was added portionwise. After $6 \mathrm{~h} \mathrm{H}_{2} \mathrm{O}$ $(200 \mathrm{~mL}$ ) was added, phases were separated, aqueous phase was extracted with dichloromethane (2 $x 200 \mathrm{~mL})$, combined organic phases were washed with $\mathrm{Na}_{2} \mathrm{~S}_{2} \mathrm{O}_{3}$ aq $(200 \mathrm{~mL}, 10 \% \mathrm{w} / \mathrm{v}), \mathrm{H}_{2} \mathrm{O}(200 \mathrm{~mL})$, brine $\left(200 \mathrm{~mL}\right.$ ), and dried over anhydrous $\mathrm{MgSO}_{4}$. Mixture was filtered, evaporated, and separated with column chromatography (eluent: dichloromethane to dichloromethane/ethyl acetate 3:1) yielding $2 \mathbf{i}(8.79 \mathrm{~g}, 30.8 \mathrm{mmol}, \mathbf{8 9 \%}$ over 2 steps) as white crystals.<smiles>O=S(=O)(CC1CCCCC1)c1ccccc1</smiles>

cyclohexylmethyl phenyl selenone, $\mathbf{2} \mathbf{i}$

white crystals, $\mathrm{mp} 121.5-123.0^{\circ} \mathrm{C}$ eluent: dichloromethane to dichloromethane/ethyl acetate 3:1 $\mathrm{Rf}=0.70$ (dichloromethane/methanol 9:1)

${ }^{1} \mathbf{H}$ NMR (400 MHz, $\left.\mathrm{CDCl}_{3}\right): \delta=7.96-7.90(\mathrm{~m}, 2 \mathrm{H}), 7.69-7.63(\mathrm{~m}, 1 \mathrm{H})$, $7.63-7.56(\mathrm{~m}, 2 \mathrm{H}), 3.34(\mathrm{~d}, J=6.8 \mathrm{~Hz}, 2 \mathrm{H}), 2.24-2.08(\mathrm{~m}, 1 \mathrm{H}), 1.93-$ $1.87(\mathrm{~m}, 2 \mathrm{H}), 1.73-1.56(\mathrm{~m}, 3 \mathrm{H}), 1.32-1.18(\mathrm{~m}, 2 \mathrm{H}), 1.16-0.99(\mathrm{~m}, 3 \mathrm{H})$.

${ }^{13} \mathrm{C}$ NMR (100 MHz, $\left.\mathrm{CDCl}_{3}\right): \delta=142.5,134.0,130.1,126.7,66.8,33.0$, $32.7,25.5,25.4$.

HRMS (ESI): $\mathrm{m} / \mathrm{z}$ [M+Na] $]^{+}$calcd for $\mathrm{C}_{13} \mathrm{H}_{18} \mathrm{O}_{2} \mathrm{SeNa}$ : 309.0370; found: 309.0363 


\section{Syntheses of sulfonium and sulfoxonium salts (7f, $7 \mathrm{e}, 7 \mathrm{~g}$ )}

Syntheses of all sulfonium salts ${ }^{[5]}$ and oxidation of sulfonium salt to sulfoxonium salt ${ }^{[6]}$ were prepared according to the literature.

Salts $\mathbf{7 a}, \mathbf{7 b}, \mathbf{7 c}$, and $\mathbf{7 d}$ were purchased from commercial suppliers (see General Informations).

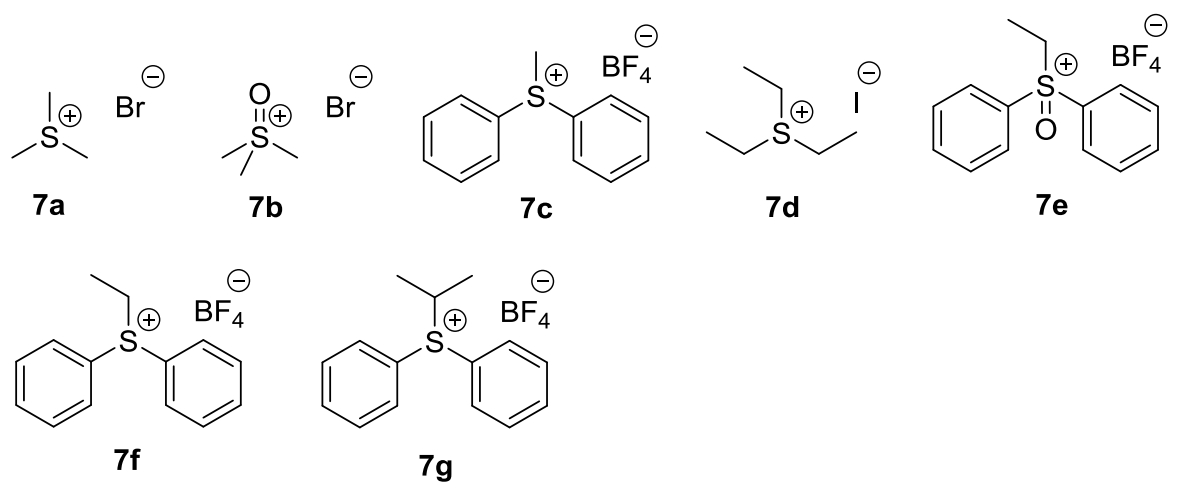




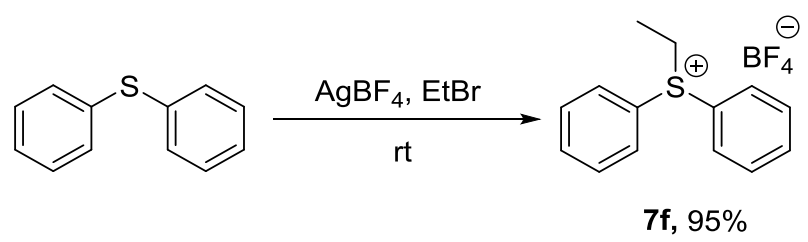

A $25 \mathrm{~mL}$ round-bottom flask was charged with $\mathrm{AgBF}_{4}(2.54 \mathrm{~g}, 13.2 \mathrm{mmol}, 1.1$ equiv.), immersed in water bath ( $\mathrm{rt}$ ) and protected from light with aluminium foil. A mixture of diphenyl sulfide $(2.27 \mathrm{~g}$, $12.1 \mathrm{mmol}, 1.0$ equiv.) and ethyl bromide $(6.54 \mathrm{~g}, 60.1 \mathrm{mmol}, 5.0$ equiv.) was added dropwise. After $64 \mathrm{~h}$ dichloromethane $(15 \mathrm{~mL})$ was added, mixture was filtered through a pad of silica gel, eluted with dichloromethane $(235 \mathrm{~mL})$, evaporated and recrystallized from dichloromethane/diethyl ether mixture yielding $7 \mathrm{f}$ ( $3.49 \mathrm{~g}, 11.6 \mathrm{mmol}, \mathbf{9 5 \%})$ as a white solid.

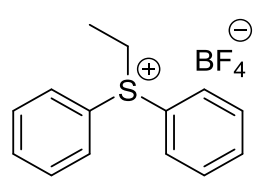

diphenylethylsulfonium tetrafluoroborate, $7 f$

white solid, $\mathrm{mp} 69.5-72.0^{\circ} \mathrm{C}$

${ }^{1}$ H NMR (400 MHz, $\left.\mathrm{CDCl}_{3}\right): \delta=7.90-7.85(\mathrm{~m}, 4 \mathrm{H}), 7.64-7.59(\mathrm{~m}, 2 \mathrm{H})$, $7.58-7.50(\mathrm{~m}, 4 \mathrm{H}), 4.06(\mathrm{q}, J=7.3 \mathrm{~Hz}, 2 \mathrm{H}), 1.35(\mathrm{t}, J=7.3 \mathrm{~Hz}, 3 \mathrm{H})$.

${ }^{13} \mathrm{C}$ NMR $\left(100 \mathrm{MHz}, \mathrm{CDCl}_{3}\right): \delta=134.4,131.3,130.4,123.9,39.7,9.4$.

${ }^{19} \mathrm{~F}$ NMR (376 $\mathrm{MHz}, \mathrm{CDCl}_{3}$ ): $\delta=-151.47$ (resonance of ${ }^{10} \mathrm{~B}$ molecule, ca. $20 \%),-151.53$.

${ }^{1} \mathrm{H}$ NMR spectrum ${ }^{[7]}$ and $\mathrm{mp}^{[8]}$ were consistent with those reported in the literature. 


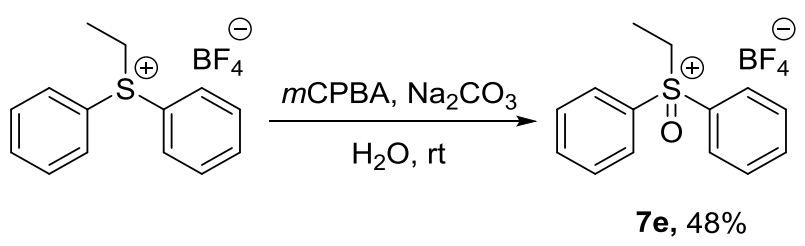

A $250 \mathrm{~mL}$ round-bottom flask was charged with diphenylethylsulfonium tetrafluoroborate, $7 \mathrm{f}(1.82 \mathrm{~g}$, $6.02 \mathrm{mmol}, 1.0$ equiv.), $\mathrm{Na}_{2} \mathrm{CO}_{3}\left(2.55 \mathrm{~g}, 24.0 \mathrm{mmol}, 4.0\right.$ equiv.), $\mathrm{H}_{2} \mathrm{O}(70 \mathrm{~mL})$ and immersed in water bath (rt). After $\mathrm{Na}_{2} \mathrm{CO}_{3}$ was dissolved $m$ CPBA ( $5.39 \mathrm{~g}, 24.0 \mathrm{mmol}$, ca. $77 \%$ purity, 4.0 equiv.) was added portionwise. After $114 \mathrm{~h} \mathrm{HCl}_{\mathrm{aq}}(10 \mathrm{~mL}, 35 \% \mathrm{w} / \mathrm{w})$ was added to $\mathrm{pH}=1$, mixture was filtered and evaporated. Acetone $(50 \mathrm{~mL})$ was added and $\mathrm{NaCl}$ precipitate was filtered out and rinsed with acetone $(50 \mathrm{~mL})$. Filtrate was evaporated yielding mixture of desired sulfoxonium salt and unreacted sulfonium salt. A $250 \mathrm{~mL}$ round-bottom flask was charged with crude mixture of product and substrate, $\mathrm{Na}_{2} \mathrm{CO}_{3}\left(0.98 \mathrm{~g}, 9.21 \mathrm{mmol}, 1.5\right.$ equiv.), $\mathrm{H}_{2} \mathrm{O}(70 \mathrm{~mL})$ and immersed in water bath ( $\left.\mathrm{rt}\right)$. After $\mathrm{Na}_{2} \mathrm{CO}_{3}$ was dissolved $m$ CPBA (2.04 g, $9.12 \mathrm{mmol}$, ca. 77\% purity, 1.5 equiv.) was added portionwise. After $120 \mathrm{~h} \mathrm{HCl}_{\mathrm{aq}}(10 \mathrm{~mL}, 35 \% \mathrm{w} / \mathrm{w})$ was added to $\mathrm{pH}=1$, mixture was filtered and evaporated. Acetone $(50 \mathrm{~mL}$ ) was added and $\mathrm{NaCl}$ precipitate was filtered off and rinsed with acetone $(50 \mathrm{~mL})$. Filtrate was evaporated, dichlormethane $(50 \mathrm{~mL})$ was added, mixture was filtered through a pad of celite, evaporated, and separated with column chromatography (eluent: dichloromethane to dichloromethane/methanol 9:1) yielding 7 e $(924 \mathrm{mg}, 2.90 \mathrm{mmol}, 48 \%)$ as a white solid.<smiles>CC[Sb](=O)(O)c1ccccc1</smiles>

diphenylethylsulfoxonium tetrafluoroborate, 7 e

white solid, $\mathrm{mp} 135.0^{\circ} \mathrm{C}$ (dec.)

eluent: dichloromethane to dichloromethane/methanol 9:1

${ }^{1} \mathrm{H}$ NMR (400 MHz, $\left.\mathrm{CDCl}_{3}\right): \delta=8.11-8.06(\mathrm{~m}, 4 \mathrm{H}), 7.88-7.82(\mathrm{~m}, 2 \mathrm{H})$, $7.77-7.71(\mathrm{~m}, 4 \mathrm{H}), 4.58(\mathrm{q}, J=7.1 \mathrm{~Hz}, 2 \mathrm{H}), 1.46(\mathrm{t}, J=7.1 \mathrm{~Hz}, 3 \mathrm{H})$.

${ }^{13} \mathrm{C} \mathrm{NMR}\left(100 \mathrm{MHz}, \mathrm{CDCl}_{3}\right): \delta=137.1,131.2,129.2,127.1,47.5,4.8$.

${ }^{19}$ F NMR (376 $\mathrm{MHz}, \mathrm{CDCl}_{3}$ ): $\delta=-151.76$ (resonance of ${ }^{10} \mathrm{~B}$ molecule, ca. $20 \%),-151.82$.

HRMS (ESI): $\mathrm{m} / \mathrm{z}\left[\mathrm{M}-\mathrm{BF}_{4}\right]^{+}$calcd for $\mathrm{C}_{14} \mathrm{H}_{15} \mathrm{OS}^{+}: 231.0844$; found: 231.0850 


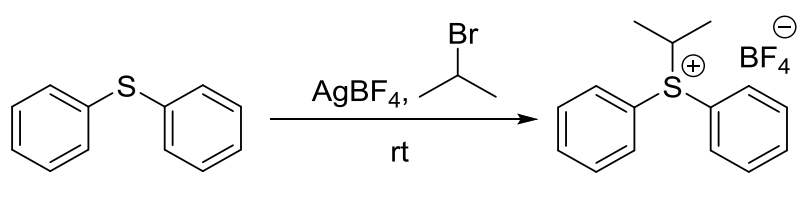

$7 g, 57 \%$

A $100 \mathrm{~mL}$ round-bottom flask was charged with $\mathrm{AgBF}_{4}(5.73 \mathrm{~g}, 29.5 \mathrm{mmol}, 0.8$ equiv.), immersed in ice-water bath (ca. $2-3{ }^{\circ} \mathrm{C}$ ) and protected from light with aluminium foil. A mixture of diphenyl sulfide ( $54.9 \mathrm{~g}, 294.6 \mathrm{mmol}, 8.2$ equiv.) and isopropyl bromide $(4.44 \mathrm{~g}, 36.1 \mathrm{mmol}, 1.0$ equiv.) was added dropwise. After $72 \mathrm{~h}$ acetone $(100 \mathrm{~mL})$ was added, mixture was filtered through a pad of silica gel, eluted with acetone $(150 \mathrm{~mL})$ and evaporated. Precipitate was dissolved in dichloromethane (250 $\mathrm{mL}$ ), filtered through a pad of celite, evaporated, and recrystallized from dichloromethane/diethyl ether mixture yielding $7 \mathrm{~g}(6.00 \mathrm{~g}, 19.0 \mathrm{mmol}, 64 \%)$ as white crystals.

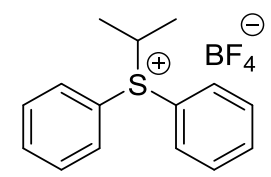

diphenylisopropylsulfonium tetrafluoroborate, $7 \mathrm{~g}$

white crystals, $\mathrm{mp} 112.5-114.0^{\circ} \mathrm{C}$

${ }^{1} \mathrm{H}$ NMR (400 MHz, $\left.\mathrm{CDCl}_{3}\right): \delta=8.13-8.04(\mathrm{~m}, 4 \mathrm{H}), 7.72-7.57(\mathrm{~m}, 6 \mathrm{H})$, 4.99 (hept, $J=6.6 \mathrm{~Hz}, 1 \mathrm{H}), 1.47(\mathrm{~d}, J=6.6 \mathrm{~Hz}, 6 \mathrm{H})$.

${ }^{13} \mathrm{C}$ NMR $\left(100 \mathrm{MHz}, \mathrm{CDCl}_{3}\right): \delta=134.7,131.5,131.3,123.8,50.5,18.4$.

${ }^{19} \mathrm{~F}$ NMR (376 $\mathrm{MHz}, \mathrm{CDCl}_{3}$ ): $\delta=-151.95$ (resonance of ${ }^{10} \mathrm{~B}$ molecule, ca. $20 \%),-152.01$.

${ }^{1} \mathrm{H}$ NMR and ${ }^{13} \mathrm{C}$ NMR spectra were consistent with those reported in the literature. ${ }^{[5]}$ 


\section{Syntheses of nitroarenes (1b, 1c, 1f, 1g)}

Nitroarenes 1a, 1d, and 1e were purchased from commercial suppliers (see General Informations).<smiles>O=[N+]([O-])c1cccc2ccccc12</smiles>

1a<smiles>O=[N+]([O-])c1cccc2c(Br)cccc12</smiles>

1b

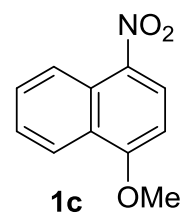

1c $\mathrm{OMe}$<smiles>O=[N+]([O-])c1ccc2ccccc2c1</smiles>

1d<smiles>O=[N+]([O-])c1ccc2ncccc2c1</smiles><smiles>CCn1cc2cc([N+](=O)[O-])ccc2n1</smiles><smiles>CCn1ncc2cc([N+](=O)[O-])ccc21</smiles> 
Synthesis of 5-bromo-1-nitronaphthalene (1b) was prepared according to the literature. ${ }^{[9]}$<smiles>O=[N+]([O-])c1cccc2c(Br)cccc12</smiles>

1b, $36 \%$

A $50 \mathrm{~mL}$ round-bottom flask equipped with liquid condenser was charged with nitronaphthalene ( $10.0 \mathrm{~g}, 56.0 \mathrm{mmol}, 1.0$ equiv.), $\mathrm{FeCl}_{2} \cdot 4 \mathrm{H}_{2} \mathrm{O}$ ( $83 \mathrm{mg}, 0.416 \mathrm{mmol}, 0.7 \% \mathrm{~mol}$ ) and warmed to $90{ }^{\circ} \mathrm{C} . \mathrm{Br}_{2}$ (9.31 g, $60.5 \mathrm{mmol}, 1.1$ equiv.) was added dropwise. After $2 \mathrm{~h}$ mixture was cooled to $\mathrm{rt}$ and $\mathrm{Na}_{2} \mathrm{~S}_{2} \mathrm{O}_{3}$ aq $(50 \mathrm{~mL}, 10 \% \mathrm{w} / \mathrm{v})$ was added. Mixture was extracted with dichloromethane $(2 \times 50 \mathrm{~mL})$, combined organic phases were washed with $\mathrm{Na}_{2} \mathrm{~S}_{2} \mathrm{O}_{3}$ aq $(50 \mathrm{~mL}, 10 \% \mathrm{w} / \mathrm{v})$, brine $(50 \mathrm{~mL})$, and dried over anhydrous $\mathrm{MgSO}_{4}$. Mixture was filtered, evaporated, and recrystallized several times from ethanol yielding $\mathbf{1 b}$ (5.29 g, $21.0 \mathrm{mmol}, \mathbf{3 6 \%}$ ) as a yellow solid.<smiles>O=[N+]([O-])c1cccc2c(Br)cccc12</smiles>

5-bromo-1-nitronaphthalene, $\mathbf{1 b}$ yellow solid, $\mathrm{mp} 120.5-122.0^{\circ} \mathrm{C}$ $\mathrm{R}_{\mathrm{f}}=0.26$ (cyclohexane/toluene $6: 1$ )

${ }^{1} \mathrm{H}$ NMR $\left(400 \mathrm{MHz}, \mathrm{CDCl}_{3}\right): \delta=8.55(\mathrm{~d}, J=8.6 \mathrm{~Hz}, 1 \mathrm{H}), 8.42(\mathrm{~d}, J=8.7 \mathrm{~Hz}$, $1 \mathrm{H}), 8.19(\mathrm{dd}, J=7.5,1.1 \mathrm{~Hz}, 1 \mathrm{H}), 7.89(\mathrm{dd}, J=7.5,1.1 \mathrm{~Hz}, 1 \mathrm{H}), 7.62(\mathrm{dd}$, $J=8.6,7.6 \mathrm{~Hz}, 1 \mathrm{H}), 7.50(\mathrm{dd}, J=8.7,7.5 \mathrm{~Hz}, 1 \mathrm{H})$.

${ }^{13} \mathrm{C}$ NMR (100 MHz, $\left.\mathrm{CDCl}_{3}\right): \delta=147.0,133.4,132.6,131.6,129.3,126.3$, $125.4,124.3,123.4,122.8$.

${ }^{1} \mathrm{H}$ NMR, ${ }^{13} \mathrm{C}$ NMR spectra and $\mathrm{mp}$ were consistent with those reported in the literature. ${ }^{[9]}$ 


\section{Synthesis of 4-methoxy-1-nitronaphthalene (1c)}

In order to synthesize 4-methoxy-1-nitronaphthalene (1c), first 1-methoxynaphthalene (10') was synthesized.

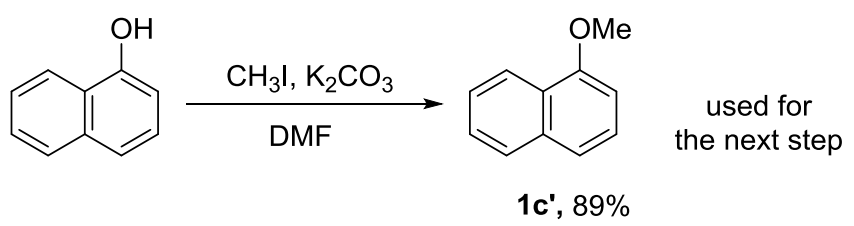

A $100 \mathrm{~mL}$ round-bottom flask was charged with 1-naphthol ( $7.21 \mathrm{~g}, 50.0 \mathrm{mmol}, 1.0$ equiv.), $\mathrm{K}_{2} \mathrm{CO}_{3}$ (13.8 g, $100.1 \mathrm{mmol}, 2.0$ equiv.) and DMF ( $25 \mathrm{~mL})$. Methyl iodide (14.8 g, $104.4 \mathrm{mmol}, 2.1$ equiv.) was added and reaction was stirred overnight. Then reaction mixture was poured onto $\mathrm{H}_{2} \mathrm{O}(200 \mathrm{~mL})$, extracted with ethyl acetate $(3 \times 100 \mathrm{~mL})$, combined organic phases were washed with $\mathrm{H}_{2} \mathrm{O}(100 \mathrm{~mL})$, brine $(100 \mathrm{~mL})$, and dried over anhydrous $\mathrm{MgSO}_{4}$. Mixture was filtered, evaporated, and crude product was separated with vacuum distillation (bath temp. $137^{\circ} \mathrm{C}, 1.8-1.9 \times 10^{-2} \mathrm{mbar}$ ) yielding $1 \mathrm{c}^{\prime}$ (7.01 g, $44.7 \mathrm{mmol}, 89 \%)$ as a pale yellow oil.<smiles>COc1cccc2ccccc12</smiles>

1-methoxynaphthalene, $\mathbf{1} \mathbf{c}^{\prime}$ pale yellow oil

$\mathrm{R}_{\mathrm{f}}=0.62$ (cyclohexane/ethyl acetate $1: 1$ )

${ }^{1} \mathrm{H}$ NMR (400 MHz, $\left.\mathrm{CDCl}_{3}\right): \delta=8.34-8.28(\mathrm{~m}, 1 \mathrm{H}), 7.87-7.80(\mathrm{~m}, 1 \mathrm{H})$, $7.55-7.48(\mathrm{~m}, 2 \mathrm{H}), 7.48-7.38(\mathrm{~m}, 2 \mathrm{H}), 6.83(\mathrm{dd}, J=7.4,1.2 \mathrm{~Hz}, 1 \mathrm{H}), 4.02$ $(\mathrm{s}, 3 \mathrm{H})$.

${ }^{13} \mathrm{C}$ NMR (100 MHz, $\left.\mathrm{CDCl}_{3}\right): \delta=155.4,134.4,127.4,126.3,125.8,125.6$, $125.1,121.9,120.2,103.7,55.4$.

4-methoxy-1-nitronaphthalene (1c) was prepared according to the literature for nitration of 2-methoxynaphthalene. ${ }^{[10]}$<smiles>COc1ccc([N+](=O)[O-])c2ccccc12</smiles> 


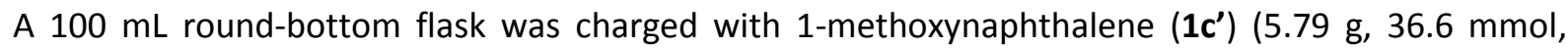
1.0 equiv.), acetic anhydride $\left(35 \mathrm{~mL}\right.$ ) and immersed in ice/water bath (ca. $\left.2-3{ }^{\circ} \mathrm{C}\right) . \mathrm{HNO}_{3}(2.27 \mathrm{~g}$, $35.9 \mathrm{mmol}, 1.0$ equiv., concd) was added dropwise (reaction mixture temperature was kept below $\left.10^{\circ} \mathrm{C}\right)$. After $1 \mathrm{~h}$ reaction mixture was poured onto ice/water mixture $(300 \mathrm{~mL})$, aqueous phase was separated from precipitate. Precipitate was dissolved in ethyl acetate $(100 \mathrm{~mL})$, resulted organic phase was washed with $\mathrm{H}_{2} \mathrm{O}(50 \mathrm{~mL})$, brine $(50 \mathrm{~mL})$ and dried over anhydrous $\mathrm{MgSO}_{4}$. Mixture was filtered, evaporated, and separated with column chromatography (eluent: cyclohexane to cyclohexane/ethyl acetate 5:1) yielding $1 \mathrm{c}(3.38 \mathrm{~g}, 6.63 \mathrm{mmol}, \mathbf{5 4 \%})$ as a yellow solid.<smiles>COc1ccc([N+](=O)[O-])c2ccccc12</smiles>

4-methoxy-1-nitronaphthalene, $1 \mathrm{c}$

yellow solid, $\mathrm{mp} 81.0-84.0^{\circ} \mathrm{C}$

eluent: cyclohexane to cyclohexane/ethyl acetate 5:1

$\mathrm{R}_{\mathrm{f}}=0.13$ (cyclohexane/ethyl acetate 20:1)

${ }^{1} \mathrm{H}$ NMR $\left(400 \mathrm{MHz}, \mathrm{CDCl}_{3}\right): \delta=8.72(\mathrm{~d}, J=8.8 \mathrm{~Hz}, 1 \mathrm{H}), 8.33(\mathrm{~d}, J=8.7 \mathrm{~Hz}$, $1 \mathrm{H}), 8.30(\mathrm{~d}, J=8.5 \mathrm{~Hz}, 1 \mathrm{H}), 7.73-7.63(\mathrm{~m}, 1 \mathrm{H}), 7.58-7.49(\mathrm{~m}, 1 \mathrm{H}), 6.73$ $(\mathrm{d}, J=8.7 \mathrm{~Hz}, 1 \mathrm{H}), 4.04(\mathrm{~s}, 3 \mathrm{H})$.

${ }^{13} \mathrm{C}$ NMR (100 MHz, $\left.\mathrm{CDCl}_{3}\right): \delta=160.5,139.0,129.9,127.1,126.7,126.4$, $125.4,123.3,122.7,101.8,56.2$.

${ }^{1} \mathrm{H}$ NMR, ${ }^{13} \mathrm{C}$ NMR spectra and $\mathrm{mp}$ were consistent with those reported in the literature. ${ }^{[1]}$ 
Synthesis of 1-ethyl-5-nitro- $1 \mathrm{H}$-indazole and 2-ethyl-5-nitro-2 $\mathrm{H}$-indazole was done according to the the literature. ${ }^{[12]}$<smiles>CCn1cc2cc([N+](=O)[O-])ccc2n1</smiles>

A $250 \mathrm{~mL}$ round-bottom flask was charged with 5-nitroindazole (4.90 g, $30.0 \mathrm{mmol}, 1.0$ equiv.) and dissolved in DMF (15 mL). $\mathrm{K}_{2} \mathrm{CO}_{3}(8.48 \mathrm{~g}, 61.4 \mathrm{mmol}, 2.0$ equiv.) was added and reaction mixture was immersed in water bath ( $\mathrm{rt}$ ). Ethyl bromide ( $3.94 \mathrm{~g}, 36.2 \mathrm{mmol}, 1.2$ equiv.) was added dropwise. After $4 \mathrm{~h}$ reaction mixture was quenched with $\mathrm{NH}_{4} \mathrm{Cl}_{\mathrm{aq}}(100 \mathrm{~mL}, 10 \% \mathrm{w} / \mathrm{v})$. Mixture was transferred to separatory funnel, extracted with ethyl acetate $(3 \times 100 \mathrm{~mL})$, combined organic phases were washed with $\mathrm{LiCl}_{\mathrm{aq}}(100 \mathrm{~mL}, 1.0 \mathrm{M}), \mathrm{H}_{2} \mathrm{O}(100 \mathrm{~mL})$, brine $(100 \mathrm{~mL})$, and dried over anhydrous $\mathrm{MgSO}_{4}$. Mixture was filtered, evaporated and separated with column chromatography (length $21 \mathrm{~cm}$, diameter 5.5 $\mathrm{cm}, 400 \mathrm{~mL}$ of silica gel) with eluent dichloromethane, then cyclohexane/ethyl acetate $3: 1$ to $1: 2$,

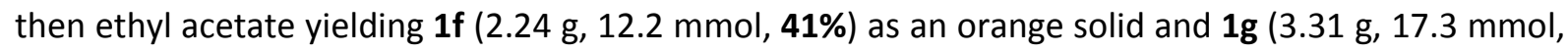
$\mathbf{5 8 \% )}$ as a pale yellow solid.<smiles>CCn1cc2cc([N+](=O)[O-])ccc2n1</smiles>

2-ethyl-5-nitro-2H-indazole, $\mathbf{1 f}$ orange solid, $\mathrm{mp} 72.5-73.5^{\circ} \mathrm{C}$

eluent: dichloromethane, then cyclohexane/ethyl acetate $3: 1$ to $1: 2$, then ethyl acetate

$\mathrm{R}_{\mathrm{f}}=0.23$ (cyclohexane/ethyl acetate $1: 1$ )

${ }^{1} \mathrm{H}$ NMR (400 MHz, $\left.\mathrm{CDCl}_{3}\right): \delta=8.56(\mathrm{dd}, J=2.2,0.8 \mathrm{~Hz}, 1 \mathrm{H}), 8.16(\mathrm{~d}$, $J=0.9 \mathrm{~Hz}, 1 \mathrm{H}), 7.92(\mathrm{dd}, J=9.5,2.2 \mathrm{~Hz}, 1 \mathrm{H}), 7.59(\mathrm{dt}, J=9.5,0.8 \mathrm{~Hz}, 1 \mathrm{H})$, $4.44(q, J=7.4 \mathrm{~Hz}, 2 \mathrm{H}), 1.57(\mathrm{t}, J=7.4 \mathrm{~Hz}, 3 \mathrm{H})$.

${ }^{13} \mathrm{C}$ NMR (100 MHz, $\left.\mathrm{CDCl}_{3}\right): \delta=149.4,142.5,126.2,119.8,119.7,119.2$, $117.9,49.0,15.3$.

${ }^{1} \mathrm{H}$ NMR, ${ }^{13} \mathrm{C}$ NMR spectra and mp were consistent with those reported in the literature. ${ }^{[13]}$ 

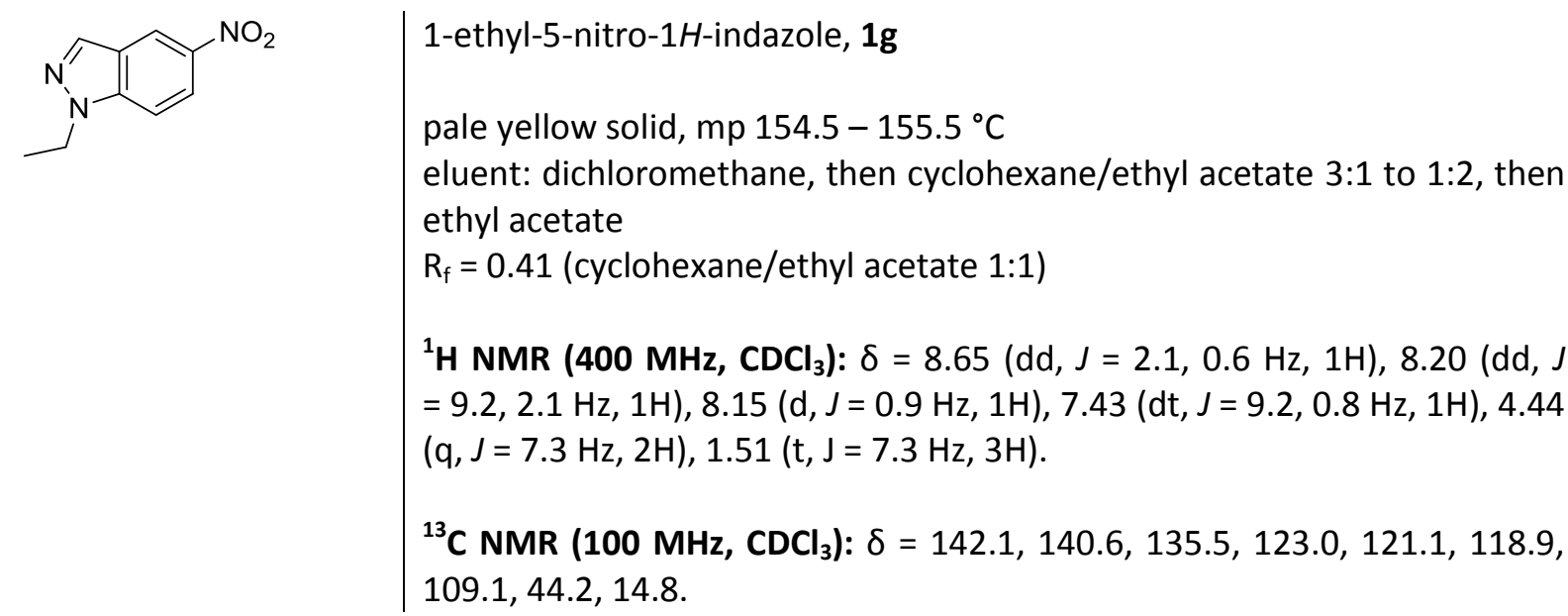

${ }^{1} \mathrm{H}$ NMR (400 MHz, $\left.\mathrm{CDCl}_{3}\right): \delta=8.65(\mathrm{dd}, J=2.1,0.6 \mathrm{~Hz}, 1 \mathrm{H}), 8.20(\mathrm{dd}, J$ $=9.2,2.1 \mathrm{~Hz}, 1 \mathrm{H}), 8.15(\mathrm{~d}, J=0.9 \mathrm{~Hz}, 1 \mathrm{H}), 7.43(\mathrm{dt}, J=9.2,0.8 \mathrm{~Hz}, 1 \mathrm{H}), 4.44$ $(q, J=7.3 \mathrm{~Hz}, 2 \mathrm{H}), 1.51(\mathrm{t}, \mathrm{J}=7.3 \mathrm{~Hz}, 3 \mathrm{H})$.

${ }^{13} \mathrm{C}$ NMR (100 MHz, $\left.\mathrm{CDCl}_{3}\right): \delta=142.1,140.6,135.5,123.0,121.1,118.9$, 109.1, 44.2, 14.8 .

${ }^{1} \mathrm{H}$ NMR, ${ }^{13} \mathrm{C}$ NMR spectra and $\mathrm{mp}$ were consistent with those reported in the literature. ${ }^{[13]}$ 


\section{Synthesis of sulfone (5)}

Synthesis of ethyl phenyl sulfone (5) was done according to the literature. ${ }^{[14]}$

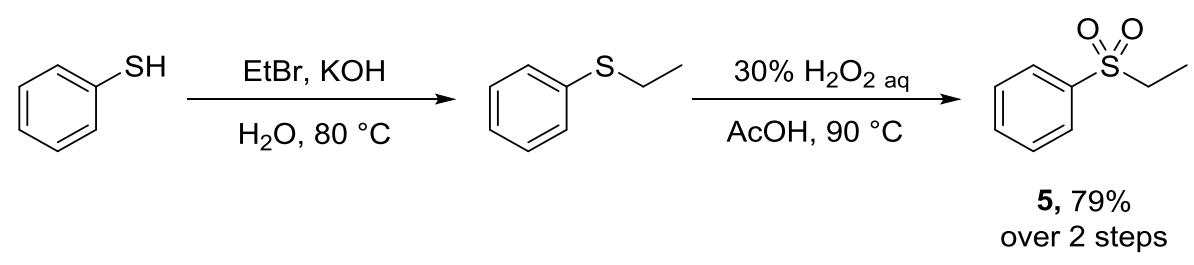

A $100 \mathrm{~mL}$ round-bottom flask was charged with thiophenol $(5.50 \mathrm{~g}, 49.9 \mathrm{mmol}, 1.0$ equiv.) and immersed in water bath (rt). Solution of $\mathrm{KOH}\left(4.21 \mathrm{~g}, 75.1 \mathrm{mmol}, 1.5\right.$ equiv.) in $\mathrm{H}_{2} \mathrm{O}(35 \mathrm{~mL})$ was added portionwise, then ethyl bromide $(6.00 \mathrm{~g}, 54.9 \mathrm{mmol}, 1.1$ equiv.) was added dropwise. Reaction mixture was heated to $80^{\circ} \mathrm{C}$. After $3 \mathrm{~h}$ reaction mixture was extracted with ethyl acetate $(3 \times 50 \mathrm{~mL})$, combined organic phases were washed with $\mathrm{H}_{2} \mathrm{O}(50 \mathrm{~mL})$, brine $(50 \mathrm{~mL})$, and dried over anhydrous $\mathrm{MgSO}_{4}$. The mixture was filtered and evaporated. Crude ethyl phenyl was placed in $100 \mathrm{~mL}$ roundbottom flask, acetic acid $(20 \mathrm{~mL})$ was added, $\mathrm{H}_{2} \mathrm{O}_{2}$ aq $(16 \mathrm{~mL}, 157.0 \mathrm{mmol}, 30 \% \mathrm{v} / \mathrm{v}, 3.1$ equiv.) was added dropwise and reaction mixture was heated to ca. $90^{\circ} \mathrm{C}$. After $3 \mathrm{~h}$ reaction mixture was poured onto water/ice ( $300 \mathrm{~mL}$ ), solid $\mathrm{Na}_{2} \mathrm{CO}_{3}$ was added to $\mathrm{pH}=7$, mixture was extracted with ethyl acetate $(3 \times 50 \mathrm{~mL})$, combined organic phases were washed with $\mathrm{Na}_{2} \mathrm{SO}_{3}$ aq $(50 \mathrm{~mL}, 10 \% \mathrm{w} / \mathrm{v})$, brine $(50 \mathrm{~mL})$, and dried over anhydrous $\mathrm{MgSO}_{4}$. Mixture was filtered, evaporated, and crude product was recrystallized form ethanol yielding 5 (6.71 g, $39.4 \mathrm{mmol}, 79 \%$ over 2 steps) as white crystals.

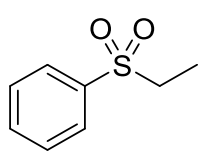

ethyl phenyl sulfone, $\mathbf{5}$

white crystals, $\mathrm{mp} 41.0-42.5^{\circ} \mathrm{C}$

$\mathrm{R}_{\mathrm{f}}=0.39$ (toluene/ethyl acetate $20: 1$ )

${ }^{1} \mathrm{H}$ NMR (400 MHz, $\left.\mathrm{CDCl}_{3}\right): \delta=7.86-7.81(\mathrm{~m}, 2 \mathrm{H}), 7.62-7.56(\mathrm{~m}, 1 \mathrm{H})$, $7.53-7.47(\mathrm{~m}, 2 \mathrm{H}), 3.06(\mathrm{q}, J=7.4 \mathrm{~Hz}, 2 \mathrm{H}), 1.20(\mathrm{t}, J=7.4 \mathrm{~Hz}, 3 \mathrm{H})$.

${ }^{13} \mathrm{C} \mathrm{NMR}\left(100 \mathrm{MHz}, \mathrm{CDCl}_{3}\right): \delta=138.3,133.5,129.1,128.0,50.4,7.3$.

${ }^{1} \mathrm{H}$ NMR, ${ }^{13} \mathrm{C}$ NMR spectra and $\mathrm{mp}$ were consistent with those reported in the literature. ${ }^{[15]}$ 


\section{Cyclopropanation of nitroarenes}

\subsection{General procedure $A\left(-40^{\circ} \mathrm{C}\right)$}

General procedure A was preferably chosen for every pair of substrates (nitroarene and carbanion precursor). For cases where at least one of substrates was insoluble under reaction conditions (at $-40{ }^{\circ} \mathrm{C}$ in DMF) reaction was carried out in ice/water bath (ca. $2-3{ }^{\circ} \mathrm{C}$, procedure $\mathrm{B}$ ), all other conditions remained unchanged.

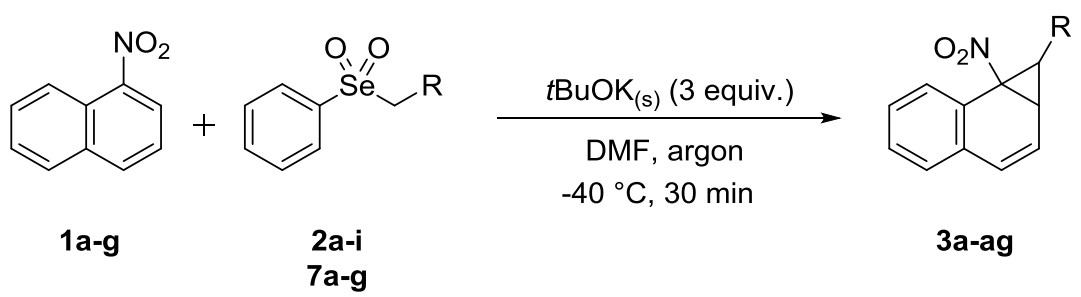

A $30 \mathrm{~mL}$ Schlenk flask was charged with nitroarene $(2.0 \mathrm{mmol}, 1.0$ equiv.), selenone or sulfonium salt ( $2.4 \mathrm{mmol}, 1.2$ equiv.) and flushed with argon. Dry DMF $(8 \mathrm{~mL})$ was added and after dissolution of substrates mixture was cooled to $-40^{\circ} \mathrm{C}$. Solid potassium tert-butoxide ( $673 \mathrm{mg}, 6.0 \mathrm{mmol}, 3.0$ equiv.) was added in one portion with stirring at $300 \mathrm{rpm}$. After $30 \mathrm{~min}$ reaction mixture was quenched with $\mathrm{NH}_{4} \mathrm{Cl}_{\mathrm{aq}}(10 \mathrm{~mL}, 10 \% \mathrm{w} / \mathrm{v})$. Mixture was transferred to separatory funnel, $\mathrm{NH}_{4} \mathrm{Cl}_{\mathrm{aq}}(50 \mathrm{~mL}, 10 \% \mathrm{w} / \mathrm{v})$ was added, mixture was extracted with ethyl acetate $(3 \times 50 \mathrm{~mL})$, combined organic phases were washed with $\mathrm{LiCl}_{\mathrm{aq}}(50 \mathrm{~mL}, 1.0 \mathrm{M}), \mathrm{H}_{2} \mathrm{O}(50 \mathrm{~mL})$, brine $(50 \mathrm{~mL})$, and dried over anhydrous $\mathrm{MgSO}_{4}$. Mixture was filtered, evaporated and separated with column chromatography (length ca. $40-45 \mathrm{~cm}$, diameter $3 \mathrm{~cm}, 250-300 \mathrm{~mL}$ of silica gel) with eluent cyclohexane/toluene 20:1 to 3:1 unless otherwise stated. In some cases recrystallization was required after column chromatography. 


\subsection{General procedure $B\left(2-3^{\circ} \mathrm{C}\right)$}

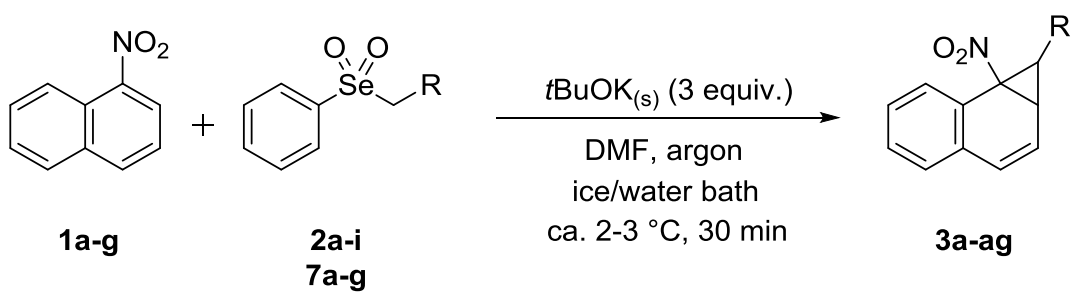

A $30 \mathrm{~mL}$ Schlenk flask was charged with nitroarene $(2.0 \mathrm{mmol}, 1.0$ equiv.), selenone or sulfonium salt ( $2.4 \mathrm{mmol}, 1.2$ equiv.) and flushed with argon. Dry DMF $(8 \mathrm{~mL})$ was added and after dissolution of substrates mixture was cooled in ice/water bath to ca. $2-3^{\circ} \mathrm{C}$. Solid potassium tert-butoxide $(673 \mathrm{mg}$, $6.0 \mathrm{mmol}, 3.0$ equiv.) was added in one portion with stirring at $300 \mathrm{rpm}$. After $30 \mathrm{~min}$ reaction mixture was quenched with $\mathrm{NH}_{4} \mathrm{Cl}_{\text {aq }}(10 \mathrm{~mL}, 10 \% \mathrm{w} / \mathrm{v})$. Mixture was transferred to separatory funnel, $\mathrm{NH}_{4} \mathrm{Cl}_{\text {aq }}(50 \mathrm{~mL}, 10 \% \mathrm{w} / \mathrm{v})$ was added, mixture was extracted with ethyl acetate ( $\left.3 \times 50 \mathrm{~mL}\right)$, combined organic phases were washed with $\mathrm{LiCl}_{\mathrm{aq}}(50 \mathrm{~mL}, 1.0 \mathrm{M}), \mathrm{H}_{2} \mathrm{O}(50 \mathrm{~mL})$, brine $(50 \mathrm{~mL})$, and dried over anhydrous $\mathrm{MgSO}_{4}$. Mixture was filtered, evaporated and separated with column chromatography (length ca. 40-45 cm, diameter $3 \mathrm{~cm}, 250-300 \mathrm{~mL}$ of silica gel) with eluent cyclohexane/toluene 20:1 to 3:1 unless otherwise stated. In some cases recrystallization was required after column chromatography. 


\subsection{Preparative experiments with selenones}

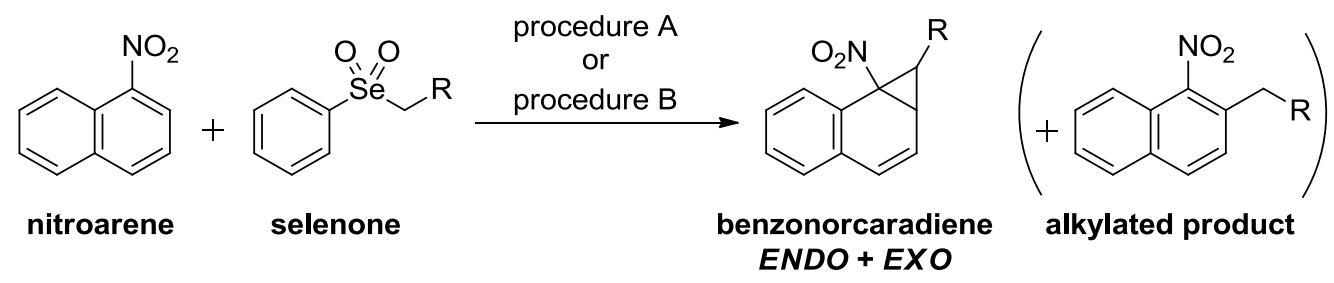

\begin{tabular}{|c|c|c|c|c|c|c|c|c|}
\hline \multirow[b]{2}{*}{ entry } & \multicolumn{3}{|c|}{ conditions } & \multicolumn{5}{|c|}{ yields [\%] } \\
\hline & nitroarene & selenone & procedure & $\begin{array}{c}\text { ENDO } \\
+ \\
\text { EXO }\end{array}$ & ENDO & EXO & $\begin{array}{c}\text { alkylated } \\
\text { product }\end{array}$ & $\begin{array}{l}\text { recovered } \\
\text { nitroarene }\end{array}$ \\
\hline 1 & $1 a$ & $2 a$ & $A$ & 2 & $-^{a}$ & $-^{a}$ & 70 & 13 \\
\hline 2 & $1 a$ & $2 b$ & $\mathrm{~A}$ & 60 & 57 & 3 & 9 & 3 \\
\hline 3 & $1 a$ & $2 c$ & $A$ & 78 & 64 & 14 & 7 & 1 \\
\hline 4 & $1 a$ & $2 d$ & $A$ & 82 & 68 & 14 & 3 & 4 \\
\hline 5 & $1 a$ & $2 e$ & $A$ & 86 & 74 & 12 & 1 & 4 \\
\hline 6 & $1 a$ & $2 f$ & $A$ & 88 & 73 & 15 & - & 4 \\
\hline 7 & $1 a$ & $2 g$ & A & 74 & 70 & 4 & 1 & 12 \\
\hline 8 & $1 a$ & $2 \mathrm{~h}$ & A & 85 & 74 & 11 & 6 & 2 \\
\hline 9 & $1 a$ & $2 \mathbf{i}$ & $B$ & 69 & 54 & 15 & 2 & - \\
\hline 10 & $1 b$ & $2 e$ & $B$ & 70 & 54 & 16 & 4 & - \\
\hline 11 & $1 b$ & $2 i$ & $B$ & 58 & 45 & 13 & 4 & 2 \\
\hline 12 & $1 c$ & $2 e$ & $A$ & 80 & 80 & $-^{c}$ & 1 & 4 \\
\hline 13 & $1 c$ & $2 f$ & $A$ & 97 & 87 & 10 & - & 3 \\
\hline 14 & $1 c$ & $2 \mathrm{~h}$ & $A$ & 92 & 82 & 10 & 2 & 1 \\
\hline 15 & $1 d$ & $2 b$ & $A$ & 75 & 71 & 4 & 1 & 9 \\
\hline 16 & 1d & $2 c$ & $A$ & 93 & 44 & 49 & 1 & 2 \\
\hline 17 & 1d & $2 e$ & A & 91 & 49 & 42 & - & 7 \\
\hline 18 & 1d & $2 f$ & $A$ & 91 & 50 & 41 & - & 6 \\
\hline 19 & 1d & $2 \mathrm{~g}$ & A & 56 & 46 & 10 & - & 9 \\
\hline 20 & 1d & $2 \mathrm{~h}$ & A & 74 & 74 & $-{ }^{b}$ & 6 & 6 \\
\hline 21 & 1d & $2 i$ & B & 99 & 54 & 45 & - & 1 \\
\hline 22 & $1 \mathrm{e}$ & $2 e$ & A & 66 & 66 & $-^{d}$ & - & 22 \\
\hline 23 & $1 \mathrm{e}$ & $2 \mathrm{~h}$ & A & 53 & 53 & $-b$ & 8 & 23 \\
\hline 24 & 1e & $2 i$ & $B$ & 83 & 75 & 8 & - & 4 \\
\hline 25 & If & $2 e$ & A & 81 & 81 & $-{ }^{b}$ & - & 6 \\
\hline 26 & $1 \mathrm{~g}$ & $2 e$ & $A$ & 59 & 59 & - & 1 & 13 \\
\hline 27 & $1 \mathrm{~g}$ & $2 f$ & $A$ & 92 & 89 & 3 & - & 1 \\
\hline
\end{tabular}

a - only one isomer of benzonorcaradiene exists

$\mathrm{b}$ - exo isomer was isomerized to endo by stirring its solution in toluene for $24 \mathrm{~h}$ in $90^{\circ} \mathrm{C}$ (due to problems with separation of endo isomer from alkylated product).

c $-<5 \%$ of exo isomer, which was inseparable from impurities.

$\mathrm{d}$-exo isomer spontaneously isomerized into endo isomer during attempt of recrystallization (crystals of endo isomer were formed). 


\subsection{Preparative experiments with sulfonium salts}

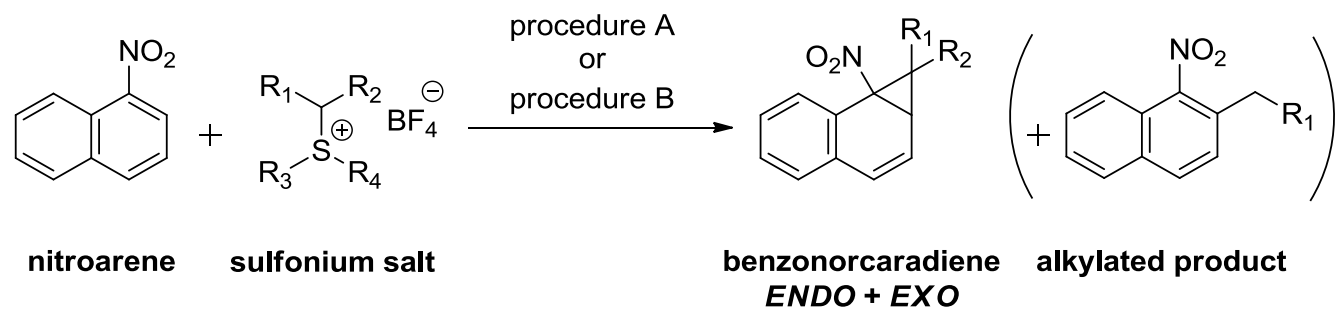

\begin{tabular}{|c|c|c|c|c|c|c|c|c|}
\hline \multirow[b]{2}{*}{ entry } & \multicolumn{3}{|c|}{ conditions } & \multicolumn{5}{|c|}{ yield [\%] } \\
\hline & nitroarene & $\begin{array}{l}\text { sulfonium } \\
\text { salt }\end{array}$ & procedure & $\begin{array}{c}\text { ENDO } \\
+ \\
\text { EXO }\end{array}$ & ENDO & EXO & $\begin{array}{l}\text { alkylated } \\
\text { product }\end{array}$ & $\begin{array}{l}\text { recovered } \\
\text { nitroarene }\end{array}$ \\
\hline 1 & 1a & $7 a$ & A & 0 & $-^{a}$ & $-{ }^{a}$ & 60 & 22 \\
\hline 2 & 1a & $7 b$ & A & 1 & $-^{a}$ & $-^{a}$ & 32 & 25 \\
\hline 3 & $1 a$ & $7 c$ & A & 49 & $-^{a}$ & $-^{a}$ & 45 & 1 \\
\hline 4 & 1a & $7 d$ & A & 2 & 2 & - & 31 & 16 \\
\hline 5 & $1 a$ & $7 e$ & A & 2 & 2 & - & - & 89 \\
\hline 6 & $1 a$ & $7 f$ & A & 71 & 33 & 38 & 13 & - \\
\hline 7 & $1 a$ & $7 g$ & A & 84 & $-^{a}$ & $-^{a}$ & - & 13 \\
\hline 8 & 1d & $7 g$ & A & 87 & $-^{a}$ & $-{ }^{a}$ & - & - \\
\hline 9 & 1c & $7 g$ & A & 97 & $-^{a}$ & $-^{a}$ & - & - \\
\hline 10 & $1 e$ & $7 g$ & B & 60 & $-^{a}$ & $-{ }^{a}$ & - & 2 \\
\hline 11 & 1f & $7 g$ & A & 99 & $-^{a}$ & $-^{a}$ & - & - \\
\hline 12 & $1 \mathrm{~g}$ & $7 g$ & B & 83 & $-{ }^{a}$ & $-{ }^{a}$ & - & 3 \\
\hline
\end{tabular}

$\mathrm{a}-$ only one isomer of benzonorcaradiene exists 


\subsection{General informations concerning synthesis of benzonorcaradienes}

Herein we present general observations that may be helpful in repeating syntheses of benzonorcaradienes:

- Solid $t$ BuOK works very well in the cyclopropanation reaction, due to it is added in excess ( 3 equiv.) and being slowly dissolved in the reaction mixture (a more convenient solution of $t$ BuOK in THF works worse)

- Solid tBuOK was weighed quickly on air, without precautions against humidity and air

- In most cases it is crucial to use cyclohexane/toluene mixture as eluent in column chromatography, other popular eluents like cyclohexane, cyclohexane/ethyl acetate, cyclohexane/diethyl ether are much less efficient (even if at TLC they might provide better separation)

- Exo isomer is eluted faster than endo isomer (in cyclohexane/toluene), except of case of heteroaromatics

- Benzonorcaradienes synthesised from 4-methoxy-1-nitronaphthalene (1c), 6-nitroquinoline (1e), 2-ethyl-5-nitro- $2 H$-indazole (1f) and 1-ethyl-5-nitro- $1 H$-indazole $(\mathbf{1 g})$ are more polar, and were eluted with more polar eluents (given below characterization data for each case)

- The reactions carried out according to the general procedures are heterogenic, with solid $t B u O K$ and liquid solution of other reactants. Therefore rate of dissolution of base is expectedly important for the reaction course. Our reactions were carried out in Schlenk flasks $(\varnothing 30 \times 92 \mathrm{~mm}$ ) stirred with magnetic bar (fish, $\varnothing 10 \times 20 \mathrm{~mm}$ ) at $300 \mathrm{rpm}$. 


\subsection{Characterization of benzonorcaradienes}

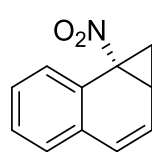

3a

Yield: $8 \mathrm{mg}(0.04 \mathrm{mmol}$ at $2.02 \mathrm{mmol}$ scale from $\mathbf{2 b}, \mathbf{2 \%})$

$3 \mathrm{mg}(0.02 \mathrm{mmol}$ at $2.01 \mathrm{mmol}$ scale from $7 \mathrm{~b}, 1 \%)$

$186 \mathrm{mg}(0.99 \mathrm{mmol}$ at $2.03 \mathrm{mmol}$ scale from $7 \mathrm{c}, 49 \%)$

yellow oil

eluent: cyclohexane/toluene 20:1 to $3: 1$

$\mathrm{R}_{\mathrm{f}}=0.25$ (cyclohexane/toluene $3: 1$ )

${ }^{1}$ H NMR (400 MHz, $\left.\mathrm{CDCl}_{3}\right): \delta=7.66-7.61(\mathrm{~m}, 1 \mathrm{H}), 7.38-7.28(\mathrm{~m}, 2 \mathrm{H})$, $7.23-7.19(\mathrm{~m}, 1 \mathrm{H}), 6.45(\mathrm{~d}, J=9.8 \mathrm{~Hz}, 1 \mathrm{H}), 6.22(\mathrm{dd}, J=9.8,5.1 \mathrm{~Hz}, 1 \mathrm{H})$, $2.93(\mathrm{dd}, J=11.1,5.2 \mathrm{~Hz}, 1 \mathrm{H}), 2.79(\mathrm{ddd}, J=11.1,7.1,5.1 \mathrm{~Hz}, 1 \mathrm{H}), 0.50$ (dd, $J=7.1,5.2 \mathrm{~Hz}, 1 \mathrm{H}$ ).

${ }^{13} \mathrm{C}$ NMR $\left(100 \mathrm{MHz}, \mathrm{CDCl}_{3}\right): \delta=129.6,128.4,128.03,127.95,127.6,126.8$, $125.7,124.9,64.1,30.9,15.8$.

MS (EI): m/z (\%) = $187\left(11,[M]^{+}\right), 141\left(100,\left[M-N_{2}\right]^{+}\right)$

HRMS (EI): $\mathrm{m} / \mathrm{z}[\mathrm{M}]^{+}$calcd for $\mathrm{C}_{11} \mathrm{H}_{9} \mathrm{NO}_{2}$ : 187.0633; found: 187.0637 


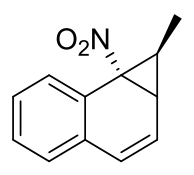

endo-3b

Yield: $117 \mathrm{mg}(0.58 \mathrm{mmol}$ at $1.02 \mathrm{mmol}$ scale from $\mathbf{2 b}, \mathbf{5 7 \%})$

$8 \mathrm{mg}(0.04 \mathrm{mmol}$ at $2.02 \mathrm{mmol}$ scale from $7 \mathrm{~d}, 2 \%)$

$9 \mathrm{mg}(0.05 \mathrm{mmol}$ at $2.02 \mathrm{mmol}$ scale from $7 \mathrm{e}, 2 \%)$

$131 \mathrm{mg}$ (0.65 mmol at $2.01 \mathrm{mmol}$ scale form 7f, 33\%)

pale yellow solid; $\mathrm{mp} 54.0-55.5^{\circ} \mathrm{C}$

eluent: cyclohexane/toluene $20: 1$ to $3: 1$

$R_{f}=0.26$ (cyclohexane/toluene $3: 1$ )

${ }^{1} \mathrm{H}$ NMR $\left(400 \mathrm{MHz}, \mathrm{CDCl}_{3}\right): \delta=7.62-7.56(\mathrm{~m}, 1 \mathrm{H}), 7.37-7.29(\mathrm{~m}, 2 \mathrm{H})$, $7.24-7.18(\mathrm{~m}, 1 \mathrm{H}), 6.60(\mathrm{~d}, J=9.8 \mathrm{~Hz}, 1 \mathrm{H}), 6.00(\mathrm{dd}, J=9.8,5.1 \mathrm{~Hz}, 1 \mathrm{H})$, $3.01-2.89(\mathrm{~m}, 2 \mathrm{H}), 0.50(\mathrm{~d}, J=6.1 \mathrm{~Hz}, 3 \mathrm{H})$.

${ }^{13} \mathrm{C}$ NMR $\left(100 \mathrm{MHz}, \mathrm{CDCl}_{3}\right): \delta=131.6,129.7,128.3,128.2,127.7,127.5$, $124.1,121.4,68.7,35.4,15.2,7.1$.

MS (EI): m/z (\%) = $201\left(2,[M]^{+}\right), 186\left(1,\left[M-\mathrm{CH}_{3}\right]^{+}\right), 155\left(100,\left[\mathrm{M}-\mathrm{NO}_{2}\right]^{+}\right)$

HRMS (EI): $\mathrm{m} / \mathrm{z}[\mathrm{M}]^{+}$calcd for $\mathrm{C}_{12} \mathrm{H}_{11} \mathrm{NO}_{2}$ : 201.0790; found: 201.0786

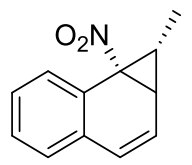

\section{exo-3b}

Yield: $152 \mathrm{mg}$ ( $0.75 \mathrm{mmol}$ at $2.01 \mathrm{mmol}$ scale from $7 \mathrm{f}, 38 \%$ )

yellowish oil

eluent: cyclohexane/toluene 20:1 to $3: 1$

$\mathrm{R}_{\mathrm{f}}=0.38$ (cyclohexane/toluene $3: 1$ )

${ }^{1} \mathrm{H}$ NMR (400 MHz, $\left.\mathrm{CDCl}_{3}\right): \delta=7.90-7.84(\mathrm{~m}, 1 \mathrm{H}), 7.35-7.26(\mathrm{~m}, 2 \mathrm{H})$, $7.20-7.15(\mathrm{~m}, 1 \mathrm{H}), 6.35(\mathrm{~d}, J=9.7 \mathrm{~Hz}, 1 \mathrm{H}), 6.24(\mathrm{dd}, J=9.7,5.3 \mathrm{~Hz}, 1 \mathrm{H})$, $2.71(\mathrm{dd}, J=6.7,5.4 \mathrm{~Hz}, 1 \mathrm{H}), 1.38(\mathrm{~d}, J=6.4 \mathrm{~Hz}, 3 \mathrm{H}), 0.79(\mathrm{p}, J=6.4 \mathrm{~Hz}$, $1 \mathrm{H})$.

${ }^{13} \mathrm{C}$ NMR $\left(100 \mathrm{MHz}, \mathrm{CDCl}_{3}\right): \delta=129.8,128.6,128.2,127.9,127.6,125.7$, $124.9,124.8,71.7,32.6,23.2,13.4$.

MS (EI): m/z (\%) = $201\left(3,[M]^{+}\right), 186\left(1,\left[M-\mathrm{CH}_{3}\right]^{+}\right), 155\left(100,\left[\mathrm{M}-\mathrm{NO}_{2}\right]^{+}\right)$

HRMS (EI): $\mathrm{m} / \mathrm{z}$ [M] ${ }^{+}$calcd for $\mathrm{C}_{12} \mathrm{H}_{11} \mathrm{NO}_{2}$ : 201.0790; found: 201.0784 


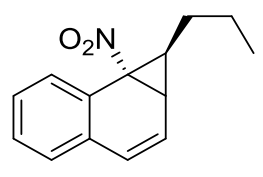

endo-3c

Yield: $295 \mathrm{mg}$ (1.29 mmol at $2.01 \mathrm{mmol}$ scale, 64\%)

pale yellow oil

eluent: cyclohexane/toluene 20:1 to 3:1

$\mathrm{R}_{\mathrm{f}}=0.32$ (cyclohexane/toluene $3: 1$ )

${ }^{1} \mathbf{H}$ NMR (400 MHz, $\left.\mathrm{CDCl}_{3}\right): \delta=7.62-7.56(\mathrm{~m}, 1 \mathrm{H}), 7.37-7.29(\mathrm{~m}, 2 \mathrm{H})$, $7.23-7.17(\mathrm{~m}, 1 \mathrm{H}), 6.57(\mathrm{~d}, J=9.8 \mathrm{~Hz}, 1 \mathrm{H}), 6.03(\mathrm{dd}, J=9.8,5.0 \mathrm{~Hz}, 1 \mathrm{H})$, $2.96-2.80(\mathrm{~m}, 2 \mathrm{H}), 1.28-1.13(\mathrm{~m}, 2 \mathrm{H}), 0.78-0.62(\mathrm{~m}, 5 \mathrm{H})$

${ }^{13} \mathrm{C}$ NMR (100 MHz, $\left.\mathrm{CDCl}_{3}\right): \delta=131.6,129.8,128.2,127.9,127.5,127.4$, $124.2,121.7,68.3,34.9,24.0,21.3,20.7,13.5$.

MS (EI): $m / z \quad(\%)=229\left(3,[M]^{+}\right), 183\left(22,\left[M-N_{2}\right]^{+}\right), 141(100$, $\left[\mathrm{M}-\mathrm{NO}_{2}-\mathrm{C}_{3} \mathrm{H}_{6}\right]^{+}$)

HRMS (EI): $\mathrm{m} / \mathrm{z}[\mathrm{M}]^{+}$calcd for $\mathrm{C}_{14} \mathrm{H}_{15} \mathrm{NO}_{2}:$ 229.1103; found: 229.1098

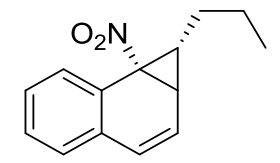

exo-3c

Yield: $64 \mathrm{mg}(0.28 \mathrm{mmol}$ at $2.01 \mathrm{mmol}$ scale, 14\%)

yellowish oil

eluent: cyclohexane/toluene $20: 1$ to $3: 1$

$\mathrm{R}_{\mathrm{f}}=0.45$ (cyclohexane/toluene $3: 1$ )

${ }^{1} \mathrm{H}$ NMR $\left(400 \mathrm{MHz}, \mathrm{CDCl}_{3}\right): \delta=7.92-7.85(\mathrm{~m}, 1 \mathrm{H}), 7.34-7.25(\mathrm{~m}, 2 \mathrm{H})$, $7.20-7.14(\mathrm{~m}, 1 \mathrm{H}), 6.36(\mathrm{~d}, J=9.6 \mathrm{~Hz}, 1 \mathrm{H}), 6.24(\mathrm{dd}, J=9.6,5.3 \mathrm{~Hz}, 1 \mathrm{H})$, $2.75(\mathrm{dd}, J=6.9,5.3 \mathrm{~Hz}, 1 \mathrm{H}), 1.85-1.75(\mathrm{~m}, 1 \mathrm{H}), 1.57-1.47(\mathrm{~m}, 2 \mathrm{H})$, $1.46-1.35(\mathrm{~m}, 1 \mathrm{H}), 0.94(\mathrm{t}, J=7.3 \mathrm{~Hz}, 3 \mathrm{H}), 0.77$ (ddd, $J=7.6,6.9,6.2 \mathrm{~Hz}$, $1 \mathrm{H})$.

${ }^{13} \mathrm{C}$ NMR (100 MHz, $\left.\mathrm{CDCl}_{3}\right): \delta=129.9,128.6,128.2,128.0,127.8,126.1$, $125.2,124.9,71.3,32.0,30.4,28.6,21.7,13.8$.

MS (EI): m/z (\%) = $229\left(3,[M]^{+}\right), 183\left(24,\left[M-N_{2}\right]^{+}\right), 141(100$, $\left[\mathrm{M}-\mathrm{NO}_{2}-\mathrm{C}_{3} \mathrm{H}_{6}\right]^{+}$)

HRMS (EI): $\mathrm{m} / \mathrm{z}$ [M] ${ }^{+}$calcd for $\mathrm{C}_{14} \mathrm{H}_{15} \mathrm{NO}_{2}:$ :229.1103; found: 229.1105 


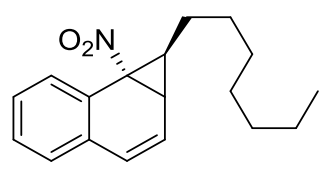

endo-3d

Yield: $390 \mathrm{mg}$ (1.37 $\mathrm{mmol}$ at $2.02 \mathrm{mmol}$ scale, $68 \%)$

colorless oil

eluent: cyclohexane/toluene 20:1 to 3:1

$\mathrm{R}_{\mathrm{f}}=0.38$ (cyclohexane/toluene $3: 1$ )

${ }^{1} \mathbf{H}$ NMR (400 MHz, $\left.\mathrm{CDCl}_{3}\right): \delta=7.63-7.57(\mathrm{~m}, 1 \mathrm{H}), 7.36-7.28(\mathrm{~m}, 2 \mathrm{H})$, $7.23-7.17(\mathrm{~m}, 1 \mathrm{H}), 6.57(\mathrm{~d}, J=9.8 \mathrm{~Hz}, 1 \mathrm{H}), 6.02(\mathrm{dd}, J=9.8,5.1 \mathrm{~Hz}, 1 \mathrm{H})$, $2.95-2.80(\mathrm{~m}, 2 \mathrm{H}), 1.28-1.01(\mathrm{~m}, 10 \mathrm{H}), 0.83(\mathrm{t}, J=7.1 \mathrm{~Hz}, 3 \mathrm{H}), 0.70(\mathrm{q}$, $J=7.0 \mathrm{~Hz}, 2 \mathrm{H})$.

${ }^{13} \mathrm{C}$ NMR (100 MHz, $\left.\mathrm{CDCl}_{3}\right): \delta=131.6,129.8,128.2,127.9,127.5,127.4$, $124.2,121.7,68.4,35.0,31.5,28.9,28.8,28.0,22.5,22.0,21.0,14.0$.

MS (EI): m/z (\%) = $285\left(1,[M]^{+}\right), 239\left(15,\left[M-N_{2}\right]^{+}\right), 141$ (100, $\left[\mathrm{M}-\mathrm{NO}_{2}-\mathrm{C}_{7} \mathrm{H}_{14}\right]^{+}$)

HRMS (EI): $\mathrm{m} / \mathrm{z}[\mathrm{M}]^{+}$calcd for $\mathrm{C}_{18} \mathrm{H}_{23} \mathrm{NO}_{2}$ : 285.1729; found: 285.1732

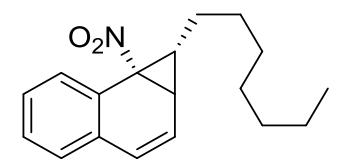

exo-3d

Yield: $78 \mathrm{mg}(0.27 \mathrm{mmol}$ at $2.02 \mathrm{mmol}$ scale, $14 \%)$

colorless oil

eluent: cyclohexane/toluene $20: 1$ to $3: 1$

$\mathrm{R}_{\mathrm{f}}=0.53$ (cyclohexane/toluene $3: 1$ )

${ }^{1}{ }_{H}$ NMR $\left(400 \mathrm{MHz}, \mathrm{CDCl}_{3}\right): \delta=7.91-7.85(\mathrm{~m}, 1 \mathrm{H}), 7.33-7.25(\mathrm{~m}, 2 \mathrm{H})$, $7.20-7.14(\mathrm{~m}, 1 \mathrm{H}), 6.35(\mathrm{~d}, J=9.6 \mathrm{~Hz}, 1 \mathrm{H}), 6.24(\mathrm{dd}, J=9.6,5.3 \mathrm{~Hz}, 1 \mathrm{H})$, $2.74(\mathrm{dd}, J=6.9,5.3 \mathrm{~Hz}, 1 \mathrm{H}), 1.85-1.76(\mathrm{~m}, 1 \mathrm{H}), 1.57-1.36(\mathrm{~m}, 3 \mathrm{H})$, $1.35-1.19(\mathrm{~m}, 8 \mathrm{H}), 0.87(\mathrm{t}, J=6.9 \mathrm{~Hz}, 3 \mathrm{H}), 0.74(\mathrm{dt}, J=6.9,6.4 \mathrm{~Hz}, 1 \mathrm{H})$.

${ }^{13} \mathrm{C}$ NMR (100 MHz, $\left.\mathrm{CDCl}_{3}\right): \delta=129.9,128.5,128.2,127.9,127.8,126.1$, 125.2, 124.9, 71.4, 32.0, 31.7, 29.2, 29.1, 28.9, 28.44, 28.42, 22.6, 14.1.

MS (EI): m/z (\%) = $285\left(1,[M]^{+}\right), 239\left(17,\left[M-N_{2}\right]^{+}\right), 141$ (100, $\left[\mathrm{M}-\mathrm{NO}_{2}-\mathrm{C}_{7} \mathrm{H}_{14}\right]^{+}$)

HRMS (EI): $\mathrm{m} / \mathrm{z}$ [M] ${ }^{+}$calcd for $\mathrm{C}_{18} \mathrm{H}_{23} \mathrm{NO}_{2}:$ :285.1729; found: 285.1725 


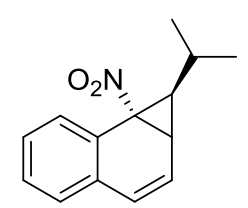

endo-3e

Yield: $349 \mathrm{mg}$ (1.51 $\mathrm{mmol}$ at $2.03 \mathrm{mmol}$ scale, $74 \%)$

pale yellow crystals, $\mathrm{mp} 95.0-96.5^{\circ} \mathrm{C}$

eluent: cyclohexane/toluene $20: 1$ to $3: 1$

$\mathrm{R}_{\mathrm{f}}=0.34$ (cyclohexane/toluene $3: 1$ )

${ }^{1} \mathbf{H}$ NMR (400 MHz, $\left.\mathrm{CDCl}_{3}\right): \delta=7.64-7.57(\mathrm{~m}, 1 \mathrm{H}), 7.37-7.28(\mathrm{~m}, 2 \mathrm{H})$, $7.22-7.17(\mathrm{~m}, 1 \mathrm{H}), 6.54(\mathrm{~d}, J=9.8 \mathrm{~Hz}, 1 \mathrm{H}), 6.07(\mathrm{dd}, J=9.8,5.3 \mathrm{~Hz}, 1 \mathrm{H})$, $2.89(\mathrm{dd}, J=11.5,5.3 \mathrm{~Hz}, 1 \mathrm{H}), 2.62(\mathrm{dd}, J=11.5,10.7 \mathrm{~Hz}, 1 \mathrm{H}), 0.86(\mathrm{~d}$, $J=6.6 \mathrm{~Hz}, 3 \mathrm{H}), 0.66(\mathrm{~d}, J=6.4 \mathrm{~Hz}, 3 \mathrm{H}), 0.60-0.47(\mathrm{~m}, 1 \mathrm{H})$.

${ }^{13} \mathrm{C}$ NMR $\left(100 \mathrm{MHz}, \mathrm{CDCl}_{3}\right): \delta=131.6,130.1,128.3,127.8,127.52,127.49$, $124.0,121.7,68.5,34.9,28.8,22.5,21.6,20.1$.

MS (EI): $m / z(\%)=229\left(1,[M]^{+}\right), 183\left(69,\left[M-N_{2}\right]^{+}\right), 141$ (100, $\left.\left[\mathrm{M}-\mathrm{NO}_{2}-\mathrm{C}_{3} \mathrm{H}_{6}\right]^{+}\right)$

HRMS (EI): $\mathrm{m} / \mathrm{z}[\mathrm{M}]^{+}$calcd for $\mathrm{C}_{14} \mathrm{H}_{15} \mathrm{NO}_{2}: 229.1103$; found: 229.1100

After column chromatography endo-3e was recrystallized by slow evaporation of its solution in dichloromethane/heptane mixture in order to remove residual contamination.

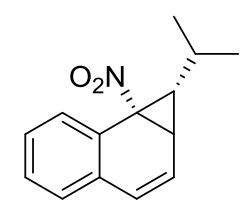

exo-3e

Yield: $60 \mathrm{mg}(0.26 \mathrm{mmol}$ at $2.03 \mathrm{mmol}$ scale, 12\%)

off-white crystals, $\mathrm{mp} 38.5-41.5^{\circ} \mathrm{C}$

eluent: cyclohexane/toluene $20: 1$ to $3: 1$

$\mathrm{R}_{\mathrm{f}}=0.43$ (cyclohexane/toluene $3: 1$ )

${ }^{1} \mathrm{H}$ NMR $\left(400 \mathrm{MHz}, \mathrm{CDCl}_{3}\right): \delta=7.99-7.90(\mathrm{~m}, 1 \mathrm{H}), 7.34-7.25(\mathrm{~m}, 2 \mathrm{H})$, $7.20-7.13(\mathrm{~m}, 1 \mathrm{H}), 6.35(\mathrm{~d}, J=9.6 \mathrm{~Hz}, 1 \mathrm{H}), 6.23(\mathrm{dd}, J=9.6,5.3 \mathrm{~Hz}, 1 \mathrm{H})$, $2.78(\mathrm{dd}, J=7.2,5.3 \mathrm{~Hz}, 1 \mathrm{H}), 1.59-1.45(\mathrm{~m}, 1 \mathrm{H}), 1.11(\mathrm{~d}, J=6.7 \mathrm{~Hz}, 3 \mathrm{H})$, $1.07(\mathrm{~d}, J=6.6 \mathrm{~Hz}, 3 \mathrm{H}), 0.56(\mathrm{dd}, J=10.2,7.2 \mathrm{~Hz}, 1 \mathrm{H})$.

${ }^{13} \mathrm{C}$ NMR $\left(100 \mathrm{MHz}, \mathrm{CDCl}_{3}\right): \delta=129.9,128.5,128.2,128.0,127.9,126.5$, $125.2,124.9,71.5,36.4,31.3,28.6,21.9,21.5$.

MS (EI): m/z (\%) = $229\left(1,[M]^{+}\right), 183\left(74,\left[M-N_{2}\right]^{+}\right), 141$ (100, $\left[\mathrm{M}-\mathrm{NO}_{2}-\mathrm{C}_{3} \mathrm{H}_{6}\right]^{+}$)

HRMS (EI): $\mathrm{m} / \mathrm{z}[\mathrm{M}]^{+}$calcd for $\mathrm{C}_{14} \mathrm{H}_{15} \mathrm{NO}_{2}:$ 229.1103; found: 229.1105 


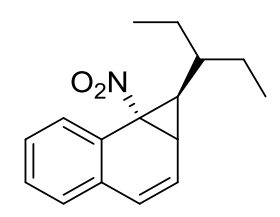

endo-3f

Yield: $375 \mathrm{mg}$ (1.46 mmol at $2.00 \mathrm{mmol}$ scale, 73\%)

off-white solid, $41.5-46.0^{\circ} \mathrm{C}$

eluent: cyclohexane/toluene $20: 1$ to $3: 1$

$\mathrm{R}_{\mathrm{f}}=0.36$ (cyclohexane/toluene $3: 1$ )

${ }^{1} \mathbf{H}$ NMR (400 MHz, $\left.\mathrm{CDCl}_{3}\right): \delta=7.62-7.56(\mathrm{~m}, 1 \mathrm{H}), 7.36-7.28(\mathrm{~m}, 2 \mathrm{H})$, $7.22-7.14(\mathrm{~m}, 1 \mathrm{H}), 6.54(\mathrm{~d}, J=9.8 \mathrm{~Hz}, 1 \mathrm{H}), 6.06(\mathrm{dd}, J=9.8,5.3 \mathrm{~Hz}, 1 \mathrm{H})$, $2.91(\mathrm{dd}, J=11.5,5.3 \mathrm{~Hz}, 1 \mathrm{H}), 2.72(\mathrm{t}, J=11.3 \mathrm{~Hz}, 1 \mathrm{H}), 1.39-1.17(\mathrm{~m}, 2 \mathrm{H})$, $1.12-0.99(\mathrm{~m}, 2 \mathrm{H}), 0.81(\mathrm{t}, J=7.5 \mathrm{~Hz}, 3 \mathrm{H}), 0.55(\mathrm{t}, J=7.5 \mathrm{~Hz}, 3 \mathrm{H}), 0.33-$ $0.21(\mathrm{~m}, 1 \mathrm{H})$.

${ }^{13} \mathrm{C}$ NMR (100 MHz, $\left.\mathrm{CDCl}_{3}\right): \delta=131.6,130.2,128.3,127.5,127.4,124.3$, $122.3,68.6,34.9,33.6,25.4,25.3,24.0,10.8,10.5$.

MS (EI): $m / z \quad(\%)=257\left(1,[M]^{+}\right), 211\left(21,\left[M-N_{2}\right]^{+}\right), 141(100$, $\left[\mathrm{M}-\mathrm{NO}_{2}-\mathrm{C}_{5} \mathrm{H}_{10}\right]^{+}$)

HRMS (EI): $\mathrm{m} / \mathrm{z}[\mathrm{M}]^{+}$calcd for $\mathrm{C}_{16} \mathrm{H}_{19} \mathrm{NO}_{2}: 257.1416$; found: 257.1418

exo-3f

Yield: $79 \mathrm{mg}(0.31 \mathrm{mmol}$ at $2.00 \mathrm{mmol}$ scale, $15 \%)$

yellowish oil

eluent: cyclohexane/toluene 20:1 to 3:1

$\mathrm{R}_{\mathrm{f}}=0.49$ (cyclohexane/toluene $3: 1$ )

${ }^{1} \mathrm{H}$ NMR (400 MHz, $\left.\mathrm{CDCl}_{3}\right): \delta=8.10-8.04(\mathrm{~m}, 1 \mathrm{H}), 7.38-7.27(\mathrm{~m}, 2 \mathrm{H})$, $7.20-7.15(\mathrm{~m}, 1 \mathrm{H}), 6.38(\mathrm{~d}, J=9.6 \mathrm{~Hz}, 1 \mathrm{H}), 6.25(\mathrm{dd}, J=9.6,5.3 \mathrm{~Hz}, 1 \mathrm{H})$, $2.97(\mathrm{dd}, J=7.9,5.3 \mathrm{~Hz}, 1 \mathrm{H}), 1.68-1.38(\mathrm{~m}, 5 \mathrm{H}), 0.98(\mathrm{t}, J=7.3 \mathrm{~Hz}, 3 \mathrm{H})$, $0.87(\mathrm{t}, J=7.4 \mathrm{~Hz}, 3 \mathrm{H}), 0.75(\mathrm{dd}, J=9.4,7.9 \mathrm{~Hz}, 1 \mathrm{H})$.

${ }^{13} \mathrm{C}$ NMR (100 MHz, $\left.\mathrm{CDCl}_{3}\right): \delta=130.0,128.4,128.1(2 \mathrm{C}), 127.9,127.8$, $125.3,125.2,69.2,38.9,35.9,32.6,26.3,25.9,11.0$ (2C).

MS (EI): $m / z \quad(\%)=257\left(1,[M]^{+}\right), 211\left(24,\left[M-N_{2}\right]^{+}\right), 141$ (100, $\left[\mathrm{M}-\mathrm{NO}_{2}-\mathrm{C}_{5} \mathrm{H}_{10}\right]^{+}$)

HRMS (EI): $\mathrm{m} / \mathrm{z}$ [M] ${ }^{+}$calcd for $\mathrm{C}_{16} \mathrm{H}_{19} \mathrm{NO}_{2}$ : 257.1416; found: 257.1418 


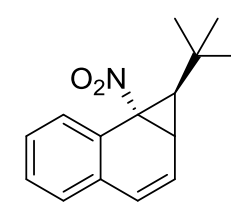

\section{endo-3g}

Yield: $345 \mathrm{mg}$ (1.43 $\mathrm{mmol}$ at $2.03 \mathrm{mmol}$ scale, $70 \%)$

white crystals, $\mathrm{mp} 79.5-80.5^{\circ} \mathrm{C}$

eluent: cyclohexane/toluene $20: 1$ to $3: 1$

$\mathrm{R}_{\mathrm{f}}=0.34$ (cyclohexane/toluene $3: 1$ )

${ }^{1} \mathbf{H}$ NMR (400 MHz, $\left.\mathrm{CDCl}_{3}\right): \delta=7.64-7.59(\mathrm{~m}, 1 \mathrm{H}), 7.39-7.30(\mathrm{~m}, 2 \mathrm{H})$, $7.19-7.13(\mathrm{~m}, 1 \mathrm{H}), 6.49(\mathrm{~d}, J=9.7 \mathrm{~Hz}, 1 \mathrm{H}), 6.17(\mathrm{dd}, J=9.7,5.4 \mathrm{~Hz}, 1 \mathrm{H})$, $2.84(\mathrm{dd}, J=12.9,5.4 \mathrm{~Hz}, 1 \mathrm{H}), 2.54(\mathrm{~d}, J=12.9 \mathrm{~Hz}, 1 \mathrm{H}), 0.73(\mathrm{~s}, 9 \mathrm{H})$.

${ }^{13} \mathrm{C}$ NMR (100 MHz, $\left.\mathrm{CDCl}_{3}\right): \delta=132.8,131.4,128.8,128.5,127.7,127.5$, $124.5,123.2,68.1,34.2,32.8,29.4,28.7$.

MS (EI): $m / z(\%)=243\left(1,[M]^{+}\right), 197\left(100,\left[M-N_{2}\right]^{+}\right), 155$ (85, $\left.\left[\mathrm{M}-\mathrm{NO}_{2}-\mathrm{C}_{3} \mathrm{H}_{6}\right]^{+}\right), 141\left(91,\left[\mathrm{M}-\mathrm{NO}_{2}-\mathrm{C}_{4} \mathrm{H}_{8}\right]^{+}\right)$

HRMS (EI): $\mathrm{m} / \mathrm{z}[\mathrm{M}]^{+}$calcd for $\mathrm{C}_{15} \mathrm{H}_{17} \mathrm{NO}_{2}:$ :243.1259; found: 243.1259

After column chromatography endo-3g was recrystallized by slow evaporation of its solution in dichloromethane/heptane mixture in order to remove residual contamination. 


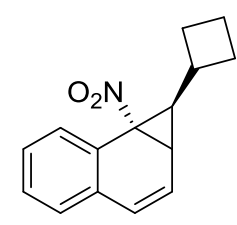

endo-3h

Yield: $360 \mathrm{mg}$ (1.49 $\mathrm{mmol}$ at $2.03 \mathrm{mmol}$ scale, $74 \%)$

pale yellow crystals, $\mathrm{mp} 86.0-87.0^{\circ} \mathrm{C}$

eluent: cyclohexane/toluene $20: 1$ to $3: 1$

$R_{f}=0.33$ (cyclohexane/toluene $3: 1$ )

${ }^{1} \mathbf{H}$ NMR (400 MHz, $\left.\mathrm{CDCl}_{3}\right): \delta=7.57-7.51(\mathrm{~m}, 1 \mathrm{H}), 7.35-7.27(\mathrm{~m}, 2 \mathrm{H})$, $7.23-7.15(\mathrm{~m}, 1 \mathrm{H}), 6.55(\mathrm{~d}, J=9.8 \mathrm{~Hz}, 1 \mathrm{H}), 6.00(\mathrm{dd}, J=9.8,5.0 \mathrm{~Hz}, 1 \mathrm{H})$, $3.02-2.89(\mathrm{~m}, 2 \mathrm{H}), 1.95-1.52(\mathrm{~m}, 5 \mathrm{H}), 1.44-1.25(\mathrm{~m}, 2 \mathrm{H})$.

${ }^{13} \mathrm{C}$ NMR $\left(100 \mathrm{MHz}, \mathrm{CDCl}_{3}\right): \delta=131.4,129.9,128.2,127.8,127.44,127.38$, $124.2,121.7,68.1,35.3,29.0,27.9,27.1,26.9,18.4$.

MS (EI): $m / z \quad(\%)=241\left(1,[M]^{+}\right), 195\left(53,\left[M-N_{2}\right]^{+}\right), 141(100$, $\left[\mathrm{M}-\mathrm{NO}_{2}-\mathrm{C}_{4} \mathrm{H}_{6}\right]^{+}$)

HRMS (EI): $\mathrm{m} / \mathrm{z}[\mathrm{M}]^{+}$calcd for $\mathrm{C}_{15} \mathrm{H}_{15} \mathrm{NO}_{2}: 241.1103$; found: 241.1110

exo-3h

Yield: $56 \mathrm{mg}(0.23 \mathrm{mmol}$ at $2.03 \mathrm{mmol}$ scale, $11 \%)$

yellowish oil

eluent: cyclohexane/toluene $20: 1$ to $3: 1$

$R_{f}=0.46$ (cyclohexane/toluene $3: 1$ )

${ }^{1}$ H NMR (400 MHz, $\left.\mathrm{CDCl}_{3}\right): \delta=7.83-7.77(\mathrm{~m}, 1 \mathrm{H}), 7.33-7.25(\mathrm{~m}, 2 \mathrm{H})$, $7.21-7.14(\mathrm{~m}, 1 \mathrm{H}), 6.36(\mathrm{~d}, J=9.6 \mathrm{~Hz}, 1 \mathrm{H}), 6.23(\mathrm{dd}, J=9.6,5.4 \mathrm{~Hz}, 1 \mathrm{H})$, $2.73(\mathrm{dd}, J=6.7,5.4 \mathrm{~Hz}, 1 \mathrm{H}), 2.32-2.11(\mathrm{~m}, 3 \mathrm{H}), 1.93-1.77(\mathrm{~m}, 4 \mathrm{H}), 0.85$ (dd, $J=8.7,6.7 \mathrm{~Hz}, 1 \mathrm{H}$ ).

${ }^{13} \mathrm{C}$ NMR (100 MHz, $\left.\mathrm{CDCl}_{3}\right): \delta=129.9,128.5,128.2,128.0,127.7,125.8$, $125.1,124.8,71.4,35.2,33.2,30.9,27.7,27.3,18.7$.

MS (EI): $m / z \quad(\%)=241\left(1,[M]^{+}\right), 195\left(49,\left[M-N_{2}\right]^{+}\right), 141(100$, $\left[\mathrm{M}-\mathrm{NO}_{2}-\mathrm{C}_{4} \mathrm{H}_{6}\right]^{+}$)

HRMS (EI): $\mathrm{m} / \mathrm{z}$ [M] ${ }^{+}$calcd for $\mathrm{C}_{15} \mathrm{H}_{15} \mathrm{NO}_{2}:$ :241.1103; found: 241.1100 


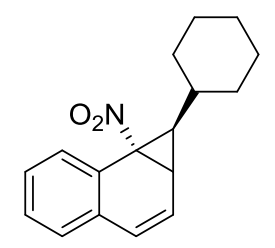

endo-3i

Yield: $293 \mathrm{mg}$ (1.09 mmol at $2.03 \mathrm{mmol}$ scale, 54\%)

white crystals, $\mathrm{mp} 137.0-138.0^{\circ} \mathrm{C}$

eluent: cyclohexane/toluene $20: 1$ to $3: 1$

$\mathrm{R}_{\mathrm{f}}=0.33$ (cyclohexane/toluene $3: 1$ )

${ }^{1} \mathbf{H}$ NMR (400 MHz, $\left.\mathrm{CDCl}_{3}\right): \delta=7.63-7.56(\mathrm{~m}, 1 \mathrm{H}), 7.36-7.28(\mathrm{~m}, 2 \mathrm{H})$, $7.23-7.16(\mathrm{~m}, 1 \mathrm{H}), 6.53(\mathrm{~d}, J=9.8 \mathrm{~Hz}, 1 \mathrm{H}), 6.05(\mathrm{dd}, J=9.8,5.3 \mathrm{~Hz}, 1 \mathrm{H})$, $2.87(\mathrm{dd}, J=11.5,5.3 \mathrm{~Hz}, 1 \mathrm{H}), 2.64(\mathrm{t}, J=11.2 \mathrm{~Hz}, 1 \mathrm{H}), 1.65-1.55(\mathrm{~m}, 1 \mathrm{H})$, $1.54-1.40(\mathrm{~m}, 3 \mathrm{H}), 1.27-1.18(\mathrm{~m}, 1 \mathrm{H}), 1.15-0.89(\mathrm{~m}, 4 \mathrm{H}), 0.75-0.60$ $(\mathrm{m}, 1 \mathrm{H}), 0.29-0.15(\mathrm{~m}, 1 \mathrm{H})$.

${ }^{13} \mathrm{C}$ NMR (100 MHz, $\left.\mathrm{CDCl}_{3}\right): \delta=131.6,130.1,128.2,127.7,127.5,127.4$, $124.0,121.8,68.2,34.5,32.0,31.4,30.6,27.0,25.9,25.7,25.4$.

MS (EI): $m / z \quad(\%)=269\left(1,[M]^{+}\right), 223\left(14,\left[M-N_{2}\right]^{+}\right), 141(100$, $\left[\mathrm{M}-\mathrm{NO}_{2}-\mathrm{C}_{6} \mathrm{H}_{10}\right]^{+}$)

HRMS (EI): $\mathrm{m} / \mathrm{z}[\mathrm{M}]^{+}$calcd for $\mathrm{C}_{17} \mathrm{H}_{19} \mathrm{NO}_{2}: 269.1416$; found: 269.1412

After column chromatography endo-3i was recrystallized by slow evaporation of its solution in dichloromethane/heptane mixture in order to remove residual contamination.

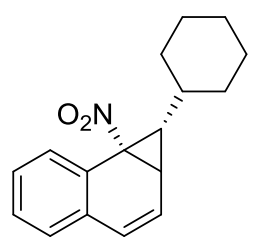

exo-3i

Yield: $81 \mathrm{mg}(0.30 \mathrm{mmol}$ at $2.03 \mathrm{mmol}$ scale, 15\%)

pale yellow solid, $63.5-65.5^{\circ} \mathrm{C}$

eluent: cyclohexane/toluene $20: 1$ to $3: 1$

$\mathrm{R}_{\mathrm{f}}=0.49$ (cyclohexane/toluene $3: 1$ )

${ }^{1} \mathrm{H}$ NMR $\left(400 \mathrm{MHz}, \mathrm{CDCl}_{3}\right): \delta=7.97-7.92(\mathrm{~m}, 1 \mathrm{H}), 7.33-7.25(\mathrm{~m}, 2 \mathrm{H})$, $7.19-7.13(\mathrm{~m}, 1 \mathrm{H}), 6.34(\mathrm{~d}, J=9.6 \mathrm{~Hz}, 1 \mathrm{H}), 6.22(\mathrm{dd}, J=9.6,5.3 \mathrm{~Hz}, 1 \mathrm{H})$, $2.79(\mathrm{dd}, J=7.3,5.3 \mathrm{~Hz}, 1 \mathrm{H}), 2.02-1.85(\mathrm{~m}, 2 \mathrm{H}), 1.79-1.58(\mathrm{~m}, 3 \mathrm{H})$, $1.34-0.95(\mathrm{~m}, 6 \mathrm{H}), 0.60(\mathrm{dd}, J=9.8,7.3 \mathrm{~Hz}, 1 \mathrm{H})$.

${ }^{13} \mathrm{C}$ NMR $\left(100 \mathrm{MHz}, \mathrm{CDCl}_{3}\right): \delta=129.9,128.5,128.1,127.92,127.89,126.5$, $125.4,124.8,71.1,37.3,34.9,32.2$ (2C), 30.8, 26.1, 25.9, 25.6.

MS (EI): $m / z \quad(\%)=269\left(1,[M]^{+}\right), 223\left(20,\left[M-N_{2}\right]^{+}\right), 141(100$, $\left[\mathrm{M}-\mathrm{NO}_{2}-\mathrm{C}_{6} \mathrm{H}_{10}\right]^{+}$)

HRMS (EI): $\mathrm{m} / \mathrm{z}[\mathrm{M}]^{+}$calcd for $\mathrm{C}_{17} \mathrm{H}_{19} \mathrm{NO}_{2}$ : 269.1416; found: 269.1416 


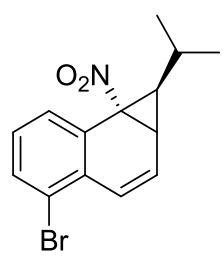

endo-3j

Yield: $335 \mathrm{mg}$ (1.09 $\mathrm{mmol}$ at $2.01 \mathrm{mmol}$ scale, 54\%)

pale yellow crystals, $\mathrm{mp} 88.5-90.5^{\circ} \mathrm{C}$

eluent: cyclohexane/toluene $20: 1$ to $3: 1$

$\mathrm{R}_{\mathrm{f}}=0.41$ (cyclohexane/toluene $3: 1$ )

${ }^{1} \mathrm{H}$ NMR (400 MHz, $\left.\mathrm{CDCl}_{3}\right): \delta=7.58(\mathrm{dd}, J=8.0,1.2 \mathrm{~Hz}, 1 \mathrm{H}), 7.54-7.50$ $(\mathrm{m}, 1 \mathrm{H}), 7.18(\mathrm{t}, J=8.0 \mathrm{~Hz}, 1 \mathrm{H}), 7.04(\mathrm{dd}, J=10.1,0.5 \mathrm{~Hz}, 1 \mathrm{H}), 6.18(\mathrm{dd}, J=$ $10.1,5.4 \mathrm{~Hz}, 1 \mathrm{H}), 2.90(\mathrm{dd}, J=11.5,5.4 \mathrm{~Hz}, 1 \mathrm{H}), 2.71-2.63(\mathrm{~m}, 1 \mathrm{H}), 0.86$ $(\mathrm{d}, J=6.6 \mathrm{~Hz}, 3 \mathrm{H}), 0.66(\mathrm{~d}, J=6.5 \mathrm{~Hz}, 3 \mathrm{H}), 0.59-0.44(\mathrm{~m}, 1 \mathrm{H})$.

${ }^{13} \mathrm{C}$ NMR (100 MHz, $\left.\mathrm{CDCl}_{3}\right): \delta=132.8,130.4,129.5,128.1,126.2,126.0$, $124.1,122.8,68.7,35.0,28.4,22.4,21.6,20.0$.

MS (EI): $\mathrm{m} / \mathrm{z}(\%)=309,307\left(1,1,[\mathrm{M}]^{+}\right), 263,261\left(15,15,\left[\mathrm{M}-\mathrm{NO}_{2}\right]^{+}\right)$, 221, $219\left(14,14,\left[\mathrm{M}-\mathrm{NO}_{2}-\mathrm{C}_{3} \mathrm{H}_{6}\right]^{+}\right), 182\left(90,\left[\mathrm{M}-\mathrm{Br}-\mathrm{NO}_{2}\right]^{+}\right), 167(100,[\mathrm{M}-\mathrm{Br}-$ $\left.\left.\mathrm{NO}_{2}-\mathrm{CH}_{3}\right]^{+}\right)$

HRMS (EI): $\mathrm{m} / \mathrm{z}[\mathrm{M}]^{+}$calcd for $\mathrm{C}_{14} \mathrm{H}_{14}{ }^{79} \mathrm{BrNO}_{2}$ : 307.0208; found: 307.0215

After column chromatography endo-3j was recrystallized by slow evaporation of its solution in dichloromethane/heptane mixture in order to remove residual contamination.

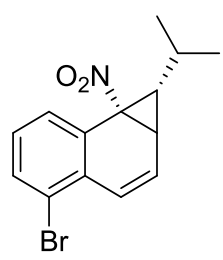

exo-3j

Yield: $99 \mathrm{mg}(0.32 \mathrm{mmol}$ at $2.01 \mathrm{mmol}$ scale, 16\%)

yellow crystals, $\mathrm{mp} 78.0-79.5^{\circ} \mathrm{C}$

eluent: cyclohexane/toluene $20: 1$ to $3: 1$

$\mathrm{R}_{\mathrm{f}}=0.53$ (cyclohexane/toluene $3: 1$ )

${ }^{1} \mathrm{H}$ NMR (400 MHz, $\left.\mathrm{CDCl}_{3}\right): \delta=7.86(\mathrm{dt}, J=8.0,1.0 \mathrm{~Hz}, 1 \mathrm{H}), 7.54(\mathrm{dd}, J=$ 8.0, $1.1 \mathrm{~Hz}, 1 \mathrm{H}), 7.15(\mathrm{t}, J=8.0 \mathrm{~Hz}, 1 \mathrm{H}), 6.86(\mathrm{dd}, J=10.0,0.7 \mathrm{~Hz}, 1 \mathrm{H}), 6.35$ (dd, $J=10.0,5.5 \mathrm{~Hz}, 1 \mathrm{H}), 2.79(\mathrm{dd}, J=7.3,5.5 \mathrm{~Hz}, 1 \mathrm{H}), 1.62-1.50(\mathrm{~m}, 1 \mathrm{H})$, $1.11(\mathrm{~d}, J=6.7 \mathrm{~Hz}, 3 \mathrm{H}), 1.07(\mathrm{~d}, J=6.5 \mathrm{~Hz}, 3 \mathrm{H}), 0.56(\mathrm{dd}, J=10.2,7.3 \mathrm{~Hz}$, $1 \mathrm{H})$.

${ }^{13} \mathrm{C}$ NMR (100 MHz, $\left.\mathrm{CDCl}_{3}\right): \delta=132.7,130.1,128.8,128.6,127.6,125.7$, $123.8,123.0,71.6,36.6,31.8,28.6,21.9,21.5$.

MS (EI): $\mathrm{m} / \mathrm{z}(\%)=309,307\left(1,1,[\mathrm{M}]^{+}\right), 263,261\left(15,16,\left[\mathrm{M}-\mathrm{NO}_{2}\right]^{+}\right)$, 221, $219\left(15,15,\left[\mathrm{M}-\mathrm{NO}_{2}-\mathrm{C}_{3} \mathrm{H}_{6}\right]^{+}\right), 182\left(87,\left[\mathrm{M}-\mathrm{Br}-\mathrm{NO}_{2}\right]^{+}\right), 167$ (100, $\left[\mathrm{M}-\mathrm{Br}-\mathrm{NO}_{2}-\mathrm{CH}_{3}\right]^{+}$)

HRMS (EI): $\mathrm{m} / \mathrm{z}[\mathrm{M}]^{+}$calcd for $\mathrm{C}_{14} \mathrm{H}_{14}{ }^{79} \mathrm{BrNO}_{2}: 307.0208$; found: 307.0207 


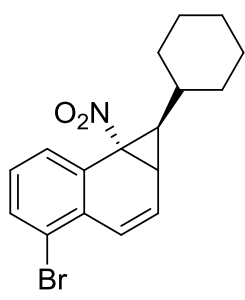

endo-3k

Yield: $316 \mathrm{mg}$ (0.91 $\mathrm{mmol}$ at $2.01 \mathrm{mmol}$ scale, 45\%)

colorless crystals, $\mathrm{mp} 125.5-126.0^{\circ} \mathrm{C}$

eluent: cyclohexane/toluene $20: 1$ to $3: 1$

$\mathrm{R}_{\mathrm{f}}=0.44$ (cyclohexane/toluene $3: 1$ )

${ }^{1} \mathrm{H}$ NMR (400 MHz, $\left.\mathrm{CDCl}_{3}\right): \delta=7.58(\mathrm{dd}, J=8.0,1.2 \mathrm{~Hz}, 1 \mathrm{H}), 7.52-7.49$ (m, $1 \mathrm{H}), 7.17(\mathrm{t}, J=8.0 \mathrm{~Hz}, 1 \mathrm{H}), 7.03(\mathrm{dd}, J=10.1,0.7 \mathrm{~Hz}, 1 \mathrm{H}), 6.16(\mathrm{dd}, J=$ 10.1, $5.3 \mathrm{~Hz}, 1 \mathrm{H}), 2.88(\mathrm{dd}, J=11.6,5.3 \mathrm{~Hz}, 1 \mathrm{H}), 2.70(\mathrm{t}, J=11.2 \mathrm{~Hz}, 1 \mathrm{H})$, $1.63-1.54(\mathrm{~m}, 1 \mathrm{H}), 1.52-1.41(\mathrm{~m}, 3 \mathrm{H}), 1.26-1.17(\mathrm{~m}, 1 \mathrm{H}), 1.14-0.89$ $(\mathrm{m}, 4 \mathrm{H}), 0.80-0.66(\mathrm{~m}, 1 \mathrm{H}), 0.26-0.13(\mathrm{~m}, 1 \mathrm{H})$.

${ }^{13} \mathrm{C}$ NMR (100 MHz, $\left.\mathrm{CDCl}_{3}\right): \delta=132.7,130.5,129.5,128.1,126.3,125.9$, $124.1,122.7,68.4,34.6,32.0,31.2,30.4,26.7,25.8,25.5,25.2$.

MS (EI): $m / z(\%)=349,347\left(1,1,[M]^{+}\right), 303,301\left(16,16,\left[M-N_{2}\right]^{+}\right), 222$ $\left(93,\left[\mathrm{M}-\mathrm{Br}-\mathrm{NO}_{2}\right]^{+}\right), 221,219\left(100,100,\left[\mathrm{M}-\mathrm{NO}_{2}-\mathrm{C}_{6} \mathrm{H}_{10}\right]^{+}\right)$

HRMS (EI): $\mathrm{m} / \mathrm{z}[\mathrm{M}]^{+}$calcd for $\mathrm{C}_{17} \mathrm{H}_{18}{ }^{79} \mathrm{BrNO}_{2}: 347.0521$; found: 347.0510

After column chromatography endo-3k was recrystallized by slow evaporation of its solution in dichloromethane/heptane mixture in order to remove residual contamination.<smiles>O=[N+]([O-])[C@]12c3cccc(Br)c3C=CC1[C@H]2C1CCCCC1</smiles>

exo-3k

Yield: $96 \mathrm{mg}(0.28 \mathrm{mmol}$ at $2.01 \mathrm{mmol}$ scale, 13\%)

pale yellow oil

eluent: cyclohexane/toluene $20: 1$ to $3: 1$

$\mathrm{R}_{\mathrm{f}}=0.57$ (cyclohexane/toluene $3: 1$ )

${ }^{1} \mathrm{H}$ NMR $\left(400 \mathrm{MHz}, \mathrm{CDCl}_{3}\right): \delta=7.85(\mathrm{~d}, J=8.0 \mathrm{~Hz}, 1 \mathrm{H}), 7.54(\mathrm{dd}, J=8.0,1.1$ $\mathrm{Hz}, 1 \mathrm{H}), 7.14(\mathrm{t}, J=8.0 \mathrm{~Hz}, 1 \mathrm{H}), 6.85(\mathrm{~d}, J=10.0 \mathrm{~Hz}, 1 \mathrm{H}), 6.33(\mathrm{dd}, J=10.0$, $5.5 \mathrm{~Hz}, 1 \mathrm{H}), 2.79(\mathrm{dd}, J=7.3,5.5 \mathrm{~Hz}, 1 \mathrm{H}), 2.01-1.84(\mathrm{~m}, 2 \mathrm{H}), 1.78-1.54$ $(\mathrm{m}, 3 \mathrm{H}), 1.35-0.94(\mathrm{~m}, 6 \mathrm{H}), 0.60(\mathrm{dd}, J=9.9,7.3 \mathrm{~Hz}, 1 \mathrm{H})$

${ }^{13} \mathrm{C}$ NMR (100 MHz, $\left.\mathrm{CDCl}_{3}\right): \delta=132.6,130.1,128.8,128.6,127.7,125.8$, $123.8,123.0,71.1,37.4,35.2,32.2$ (2C), 31.4, 26.1, 25.9, 25.6.

MS (EI): $\mathrm{m} / \mathrm{z}(\%)=349,347\left(1,1,[\mathrm{M}]^{+}\right), 303,301\left(13,13,\left[\mathrm{M}-\mathrm{NO}_{2}\right]^{+}\right), 222$ (96, $\left.\left[\mathrm{M}-\mathrm{Br}-\mathrm{NO}_{2}\right]^{+}\right), 221,219\left(100,99,\left[\mathrm{M}-\mathrm{NO}_{2}-\mathrm{C}_{6} \mathrm{H}_{10}\right]^{+}\right)$

HRMS (EI): $\mathrm{m} / \mathrm{z}[\mathrm{M}]^{+}$calcd for $\mathrm{C}_{17} \mathrm{H}_{18}{ }^{79} \mathrm{BrNO}_{2}: 347.0521$; found: 347.0527 


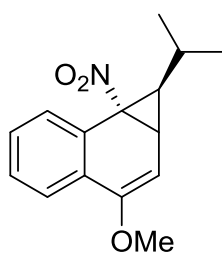

endo-3।

Yield: $417 \mathrm{mg}$ (1.61 $\mathrm{mmol}$ at $2.01 \mathrm{mmol}$ scale, $80 \%)$

white crystals, $\mathrm{mp} 111.0-113.5^{\circ} \mathrm{C}$

eluent: cyclohexane/toluene $20: 1$ to $1: 1$, then toluene

$R_{f}=0.21$ (cyclohexane/toluene $3: 1$ )

${ }^{1} \mathbf{H}$ NMR (400 MHz, $\left.\mathrm{CDCl}_{3}\right): \delta=7.79-7.72(\mathrm{~m}, 1 \mathrm{H}), 7.60-7.55(\mathrm{~m}, 1 \mathrm{H})$, $7.41-7.33(\mathrm{~m}, 2 \mathrm{H}), 5.07(\mathrm{~d}, J=5.9 \mathrm{~Hz}, 1 \mathrm{H}), 3.73(\mathrm{~s}, 3 \mathrm{H}), 2.91(\mathrm{dd}, J=10.9$, $5.9 \mathrm{~Hz}, 1 \mathrm{H}), 2.53(\mathrm{t}, J=10.8 \mathrm{~Hz}, 1 \mathrm{H}), 0.87(\mathrm{~d}, J=6.5 \mathrm{~Hz}, 3 \mathrm{H}), 0.66(\mathrm{~d}$, $J=6.2 \mathrm{~Hz}, 3 \mathrm{H}), 0.63-0.52(\mathrm{~m}, 1 \mathrm{H})$.

${ }^{13} \mathrm{C}$ NMR (100 MHz, $\left.\mathrm{CDCl}_{3}\right): \delta=151.6,130.1,129.3,128.1,127.7,125.4$, $122.1,90.2,67.7,55.0,33.2,30.2,22.4,21.3,20.1$.

MS (EI): m/z (\%) = $259\left(1,[\mathrm{M}]^{+}\right), 213\left(100,\left[\mathrm{M}-\mathrm{NO}_{2}\right]^{+}\right), 171(63$, $\left.\left[\mathrm{M}-\mathrm{NO}_{2}-\mathrm{C}_{3} \mathrm{H}_{6}\right]^{+}\right)$

HRMS (EI): $\mathrm{m} / \mathrm{z}[\mathrm{M}]^{+}$calcd for $\mathrm{C}_{15} \mathrm{H}_{17} \mathrm{NO}_{3}$ : 259.1208; found: 259.1215 


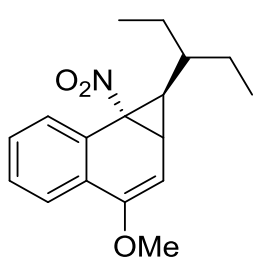

endo-3m

Yield: $500 \mathrm{mg}(1.74 \mathrm{mmol}$ at $2.00 \mathrm{mmol}$ scale, $87 \%)$

pale yellow solid, $\mathrm{mp} 60.5-62.0^{\circ} \mathrm{C}$

eluent: cyclohexane/toluene $5: 1$ to $1: 1$, then toluene

$\mathrm{R}_{\mathrm{f}}=0.24$ (cyclohexane/toluene $3: 1$ )

${ }^{1} \mathbf{H}$ NMR (400 MHz, $\left.\mathrm{CDCl}_{3}\right): \delta=7.79-7.73(\mathrm{~m}, 1 \mathrm{H}), 7.60-7.54(\mathrm{~m}, 1 \mathrm{H})$, $7.40-7.34(\mathrm{~m}, 2 \mathrm{H}), 5.07(\mathrm{~d}, J=5.9 \mathrm{~Hz}, 1 \mathrm{H}), 3.73(\mathrm{~s}, 3 \mathrm{H}), 2.93(\mathrm{dd}, J=11.0$, $5.9 \mathrm{~Hz}, 1 \mathrm{H}), 2.64(\mathrm{t}, J=11.0 \mathrm{~Hz}, 1 \mathrm{H}), 1.40-1.20(\mathrm{~m}, 2 \mathrm{H}), 1.13-1.02(\mathrm{~m}$, $2 \mathrm{H}), 0.84(\mathrm{t}, J=7.5 \mathrm{~Hz}, 3 \mathrm{H}), 0.55(\mathrm{t}, J=7.5 \mathrm{~Hz}, 3 \mathrm{H}), 0.39-0.25(\mathrm{~m}, 1 \mathrm{H})$.

${ }^{13} \mathrm{C}$ NMR (100 MHz, $\left.\mathrm{CDCl}_{3}\right): \delta=151.3,130.1,129.2,128.0,127.6,125.7$, $122.0,91.0,67.7,54.9,33.5,33.2,26.7,25.0,24.1,10.8,10.5$.

MS (EI): $m / z(\%)=287\left(1,[M]^{+}\right), 241\left(33,\left[M-N_{2}\right]^{+}\right), 171$ (100, $\left[\mathrm{M}-\mathrm{NO}_{2}-\mathrm{C}_{5} \mathrm{H}_{10}\right]^{+}$)

HRMS (EI): $\mathrm{m} / \mathrm{z}[\mathrm{M}]^{+}$calcd for $\mathrm{C}_{17} \mathrm{H}_{21} \mathrm{NO}_{3}$ : 287.1521; found: 287.1526

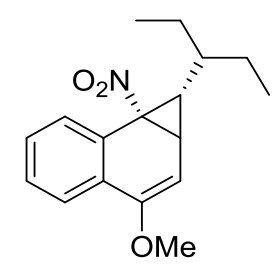

exo-3m

Yield: $57 \mathrm{mg}(0.20 \mathrm{mmol}$ at $2.00 \mathrm{mmol}$ scale, $10 \%)$

yellowish oil

eluent: cyclohexane/toluene $5: 1$ to $1: 1$, then toluene

$\mathrm{R}_{\mathrm{f}}=0.34$ (cyclohexane/toluene $3: 1$ )

${ }^{1} \mathrm{H}$ NMR $\left(400 \mathrm{MHz}, \mathrm{CDCl}_{3}\right): \delta=8.04-8.00(\mathrm{~m}, 1 \mathrm{H}), 7.76-7.72(\mathrm{~m}, 1 \mathrm{H})$, $7.39-7.30(\mathrm{~m}, 2 \mathrm{H}), 5.26(\mathrm{~d}, J=6.0 \mathrm{~Hz}, 1 \mathrm{H}), 3.72(\mathrm{~s}, 3 \mathrm{H}), 2.96(\mathrm{dd}, J=8.0$, $6.0 \mathrm{~Hz}, 1 \mathrm{H}), 1.64-1.36(\mathrm{~m}, 5 \mathrm{H}), 0.98(\mathrm{t}, J=7.3 \mathrm{~Hz}, 3 \mathrm{H}), 0.84(\mathrm{t}, J=7.4 \mathrm{~Hz}$, $3 \mathrm{H}), 0.73(\mathrm{dd}, J=9.7,8.0 \mathrm{~Hz}, 1 \mathrm{H})$.

${ }^{13} \mathrm{C}$ NMR $\left(100 \mathrm{MHz}, \mathrm{CDCl}_{3}\right): \delta=149.9,129.7,128.13,128.10,127.9,127.7$, $122.9,94.0,68.2,54.9,39.1,38.4,31.2,26.4,26.1,11.2,10.9$.

MS (EI): m/z (\%) = $287\left(1,[M]^{+}\right), 241\left(38,\left[M-N_{2}\right]^{+}\right), 171$ (100, $\left[\mathrm{M}-\mathrm{NO}_{2}-\mathrm{C}_{5} \mathrm{H}_{10}\right]^{+}$)

HRMS (EI): $\mathrm{m} / \mathrm{z}[\mathrm{M}]^{+}$calcd for $\mathrm{C}_{17} \mathrm{H}_{21} \mathrm{NO}_{3}$ : 287.1521; found: 287.1516 

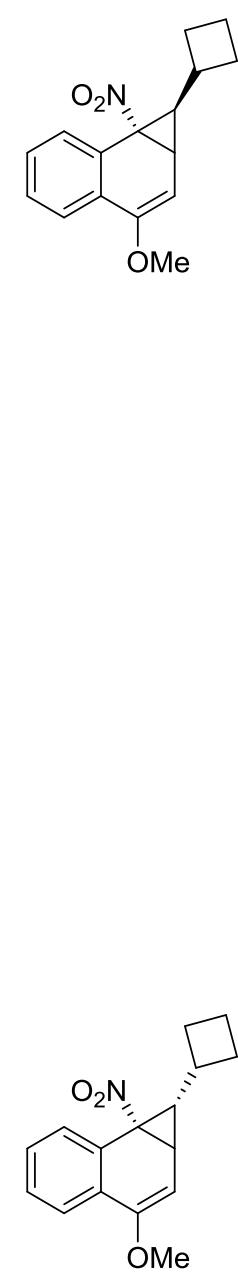

endo-3n

Yield: $443 \mathrm{mg}$ (1.63 mmol at $2.00 \mathrm{mmol}$ scale, 82\%)

off-white solid, $\mathrm{mp} 99.0-100.0^{\circ} \mathrm{C}$

eluent: cyclohexane/toluene $5: 1$ to $1: 1$, then toluene

$\mathrm{R}_{\mathrm{f}}=0.24$ (cyclohexane/toluene $3: 1$ )

${ }^{1} \mathbf{H}$ NMR (400 MHz, $\left.\mathrm{CDCl}_{3}\right): \delta=7.78-7.73(\mathrm{~m}, 1 \mathrm{H}), 7.53-7.48(\mathrm{~m}, 1 \mathrm{H})$, $7.39-7.30(\mathrm{~m}, 2 \mathrm{H}), 5.01(\mathrm{~d}, J=5.7 \mathrm{~Hz}, 1 \mathrm{H}), 3.72(\mathrm{~s}, 3 \mathrm{H}), 3.00-2.83(\mathrm{~m}$, $2 \mathrm{H}), 1.95-1.76(\mathrm{~m}, 2 \mathrm{H}), 1.74-1.53(\mathrm{~m}, 3 \mathrm{H}), 1.43-1.28(\mathrm{~m}, 2 \mathrm{H})$.

${ }^{13} \mathrm{C}$ NMR (100 MHz, $\left.\mathrm{CDCl}_{3}\right): \delta=151.7,129.9,129.0,128.0,127.7,125.7$, $122.0,90.4,67.4,54.9,33.8,29.0,28.8,27.9,26.9,18.5$.

MS (EI): $m / z(\%)=271\left(2,[M]^{+}\right), 225\left(100,\left[M-N_{2}\right]^{+}\right), 171$ (72, $\left.\left[\mathrm{M}-\mathrm{NO}_{2}-\mathrm{C}_{4} \mathrm{H}_{6}\right]^{+}\right)$

HRMS (EI): $\mathrm{m} / \mathrm{z}[\mathrm{M}]^{+}$calcd for $\mathrm{C}_{16} \mathrm{H}_{17} \mathrm{NO}_{3}:$ 271.1208; found: 271.1207

exo-3n

Yield: $55 \mathrm{mg}(0.20 \mathrm{mmol}$ at $2.00 \mathrm{mmol}$ scale, $10 \%)$

yellow oil

eluent: cyclohexane/toluene $5: 1$ to $1: 1$, then toluene

$\mathrm{R}_{\mathrm{f}}=0.33$ (cyclohexane/toluene $3: 1$ )

${ }^{1} \mathrm{H}$ NMR (400 MHz, $\left.\mathrm{CDCl}_{3}\right): \delta=7.83-7.77(\mathrm{~m}, 1 \mathrm{H}), 7.77-7.71(\mathrm{~m}, 1 \mathrm{H})$, $7.37-7.30(\mathrm{~m}, 2 \mathrm{H}), 5.26(\mathrm{~d}, J=6.0 \mathrm{~Hz}, 1 \mathrm{H}), 3.72(\mathrm{~s}, 3 \mathrm{H}), 2.74(\mathrm{dd}, J=6.8$, $6.0 \mathrm{~Hz}, 1 \mathrm{H}), 2.35-2.09(\mathrm{~m}, 3 \mathrm{H}), 1.93-1.73(\mathrm{~m}, 4 \mathrm{H}), 0.86(\mathrm{dd}, J=8.9,6.8$ $\mathrm{Hz}, 1 \mathrm{H})$.

${ }^{13} \mathrm{C}$ NMR (100 MHz, $\left.\mathrm{CDCl}_{3}\right): \delta=149.7,129.3,128.3,128.0,127.5,126.0$, $123.1,94.0,70.3,54.9,35.3(2 \mathrm{C}), 29.3,27.8,27.5,18.7$.

MS (EI): m/z (\%) = $271\left(2,[M]^{+}\right), 225\left(100,\left[M-N_{2}\right]^{+}\right), 171(61$, $\left[\mathrm{M}-\mathrm{NO}_{2}-\mathrm{C}_{4} \mathrm{H}_{6}\right]^{+}$)

HRMS (EI): $\mathrm{m} / \mathrm{z}[\mathrm{M}]^{+}$calcd for $\mathrm{C}_{16} \mathrm{H}_{17} \mathrm{NO}_{3}:$ 271.1208; found: 271.1213 


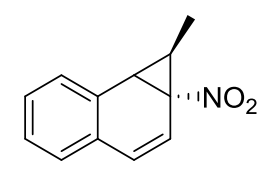

endo-30

Yield: $293 \mathrm{mg}$ (1.46 $\mathrm{mmol}$ at $2.02 \mathrm{mmol}$ scale, 71\%)

yellow crystals, $\mathrm{mp} 70.0-72.0^{\circ} \mathrm{C}$

eluent: cyclohexane/toluene $20: 1$ to $3: 1$

$\mathrm{R}_{\mathrm{f}}=0.30$ (cyclohexane/toluene $3: 1$ )

${ }^{1}$ H NMR (400 MHz, $\left.\mathrm{CDCl}_{3}\right): \delta=7.39-7.35(\mathrm{~m}, 1 \mathrm{H}), 7.32-7.23(\mathrm{~m}, 3 \mathrm{H})$, $6.70(\mathrm{dd}, J=10.2,1.0 \mathrm{~Hz}, 1 \mathrm{H}), 6.66(\mathrm{~d}, J=10.2 \mathrm{~Hz}, 1 \mathrm{H}), 3.77(\mathrm{~d}, J=11.2 \mathrm{~Hz}$, $1 \mathrm{H}), 2.69(\mathrm{dq}, J=11.2,6.4 \mathrm{~Hz}, 1 \mathrm{H}), 0.57(\mathrm{~d}, J=6.4 \mathrm{~Hz}, 3 \mathrm{H})$.

${ }^{13} \mathrm{C}$ NMR (100 MHz, $\left.\mathrm{CDCl}_{3}\right): \delta=131.1,128.92,128.90,128.4,128.1,127.5$, 127.4, 115.9, 68.0, 37.1, 18.7, 7.0.

MS (EI): m/z (\%) = $201\left(6,[M]^{+}\right), 186\left(2,\left[M-\mathrm{CH}_{3}\right]^{+}\right), 155\left(100,\left[\mathrm{M}-\mathrm{NO}_{2}\right]^{+}\right)$

HRMS (EI): $\mathrm{m} / \mathrm{z}[\mathrm{M}]^{+}$calcd for $\mathrm{C}_{12} \mathrm{H}_{11} \mathrm{NO}_{2}:$ 201.0790; found: 201.0799

After column chromatography endo-3o was recrystallized by slow evaporation of its solution in dichloromethane/heptane mixture in order to remove residual contamination. 

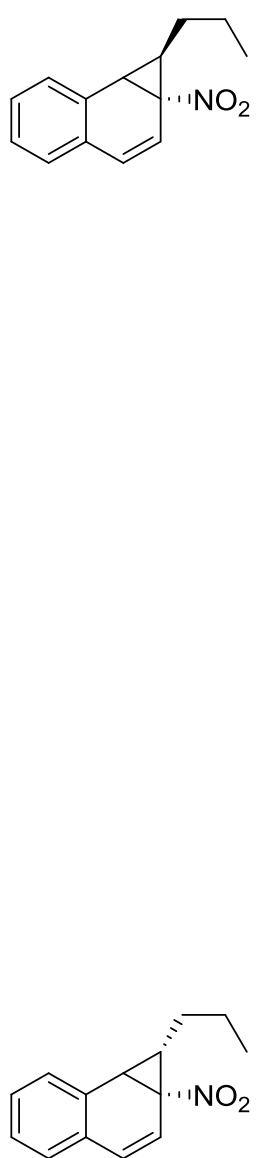

endo-3p

Yield: $202 \mathrm{mg}$ (0.88 mmol at $2.01 \mathrm{mmol}$ scale, 44\%)

yellowish oil

eluent: cyclohexane/toluene $20: 1$ to $3: 1$

$\mathrm{R}_{\mathrm{f}}=0.37$ (cyclohexane/toluene $3: 1$ )

${ }^{1} \mathbf{H}$ NMR (400 MHz, $\left.\mathrm{CDCl}_{3}\right): \delta=7.39-7.34(\mathrm{~m}, 1 \mathrm{H}), 7.31-7.22(\mathrm{~m}, 3 \mathrm{H})$, $6.73(\mathrm{dd}, J=10.1,1.2 \mathrm{~Hz}, 1 \mathrm{H}), 6.64(\mathrm{~d}, J=10.1 \mathrm{~Hz}, 1 \mathrm{H}), 3.76(\mathrm{~d}, J=11.2 \mathrm{~Hz}$, $1 \mathrm{H}), 2.63-2.52(\mathrm{~m}, 1 \mathrm{H}), 1.26-1.12(\mathrm{~m}, 2 \mathrm{H}), 0.89-0.67(\mathrm{~m}, 5 \mathrm{H})$.

${ }^{13} \mathrm{C}$ NMR (100 MHz, $\left.\mathrm{CDCl}_{3}\right): \delta=131.0,129.1,128.8,128.3,128.0,127.3$ (2C), 116.1, 67.5, 36.8, 24.3, 23.9, 21.2, 13.5.

MS (EI): m/z (\%) = $229\left(4,[M]^{+}\right), 186\left(14,\left[M-\mathrm{C}_{3} \mathrm{H}_{7}\right]^{+}\right), 183\left(19,\left[\mathrm{M}-\mathrm{NO}_{2}\right]^{+}\right)$ $141\left(100,\left[\mathrm{M}-\mathrm{NO}_{2}-\mathrm{C}_{3} \mathrm{H}_{6}\right]^{+}\right)$

HRMS (EI): $\mathrm{m} / \mathrm{z}$ [M] ${ }^{+}$calcd for $\mathrm{C}_{14} \mathrm{H}_{15} \mathrm{NO}_{2}$ : 229.1103; found: 229.1109

\section{exo-3p}

Yield: $229 \mathrm{mg}(1.00 \mathrm{mmol}$ at $2.01 \mathrm{mmol}$ scale, 49\%)

yellowish oil

eluent: cyclohexane/toluene $20: 1$ to $3: 1$

$\mathrm{R}_{\mathrm{f}}=0.46$ (cyclohexane/toluene $3: 1$ )

${ }^{1} \mathrm{H}$ NMR (400 MHz, $\left.\mathrm{CDCl}_{3}\right): \delta=7.41-7.36(\mathrm{~m}, 1 \mathrm{H}), 7.31-7.18(\mathrm{~m}, 3 \mathrm{H})$, 6.99 (dd, $J=10.0,1.3 \mathrm{~Hz}, 1 \mathrm{H}), 6.42(\mathrm{~d}, J=10.0 \mathrm{~Hz}, 1 \mathrm{H}), 3.61$ (dd, $J=7.5$, $1.3 \mathrm{~Hz}, 1 \mathrm{H}), 1.75(\mathrm{q}, J=7.3 \mathrm{~Hz}, 2 \mathrm{H}), 1.50-1.37(\mathrm{~m}, 2 \mathrm{H}), 0.97-0.84(\mathrm{~m}$, $4 \mathrm{H})$.

${ }^{13} \mathrm{C}$ NMR $\left(100 \mathrm{MHz}, \mathrm{CDCl}_{3}\right): \delta=132.2,128.9,128.4,128.3,127.9,127.1$, $125.0,121.4,69.0,35.6,33.1,28.2,21.6,13.6$.

MS (EI): m/z (\%) = $229\left(5,[M]^{+}\right), 186\left(13,\left[M-\mathrm{C}_{3} \mathrm{H}_{7}\right]^{+}\right), 183\left(20,\left[\mathrm{M}-\mathrm{NO}_{2}\right]^{+}\right)$ $141\left(100,\left[\mathrm{M}-\mathrm{NO}_{2}-\mathrm{C}_{3} \mathrm{H}_{6}\right]^{+}\right)$

HRMS (EI): $\mathrm{m} / \mathrm{z}$ [M] ${ }^{+}$calcd for $\mathrm{C}_{14} \mathrm{H}_{15} \mathrm{NO}_{2}:$ :229.1103; found: 229.1108 

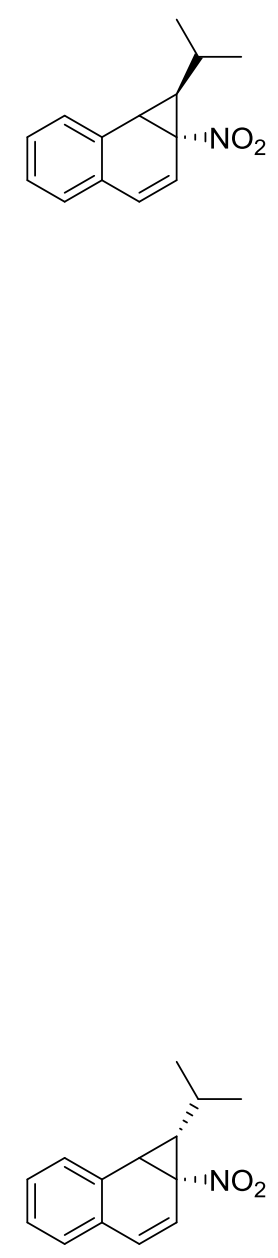

endo-3q

Yield: $224 \mathrm{mg}$ (0.98 $\mathrm{mmol}$ at $1.99 \mathrm{mmol}$ scale, $49 \%)$

white crystals, $\mathrm{mp} 88.5-89.5^{\circ} \mathrm{C}$

eluent: cyclohexane/toluene $20: 1$ to $3: 1$

$\mathrm{R}_{\mathrm{f}}=0.43$ (cyclohexane/toluene $3: 1$ )

${ }^{1}$ H NMR (400 MHz, $\left.\mathrm{CDCl}_{3}\right): \delta=7.40-7.36(\mathrm{~m}, 1 \mathrm{H}), 7.31-7.20(\mathrm{~m}, 3 \mathrm{H})$, $6.77(\mathrm{dd}, J=10.0,1.1 \mathrm{~Hz}, 1 \mathrm{H}), 6.62(\mathrm{~d}, J=10.0 \mathrm{~Hz}, 1 \mathrm{H}), 3.75(\mathrm{dd}, J=11.2$, $0.7 \mathrm{~Hz}, 1 \mathrm{H}), 2.33(\mathrm{dd}, J=11.2,10.1 \mathrm{~Hz}, 1 \mathrm{H}), 0.84(\mathrm{~d}, J=6.3 \mathrm{~Hz}, 3 \mathrm{H}), 0.75-$ $0.66(\mathrm{~m}, 1 \mathrm{H}), 0.66(\mathrm{~d}, J=5.2 \mathrm{~Hz}, 3 \mathrm{H})$.

${ }^{13} \mathrm{C}$ NMR (100 MHz, $\left.\mathrm{CDCl}_{3}\right): \delta=130.9,129.0,128.9,128.3,128.1,127.3$, $127.2,116.0,67.5,36.8,32.3,22.3,21.0,20.2$.

MS (EI): $\mathrm{m} / \mathrm{z}(\%)=229\left(8,[\mathrm{M}]^{+}\right), 186\left(71,\left[\mathrm{M}-\mathrm{C}_{3} \mathrm{H}_{7}\right]^{+}\right), 183\left(54,\left[\mathrm{M}-\mathrm{NO}_{2}\right]^{+}\right)$, $173\left(41,\left[\mathrm{M}-\mathrm{C}_{4} \mathrm{H}_{8}\right]^{+}\right), 141\left(100,\left[\mathrm{M}-\mathrm{NO}_{2}-\mathrm{C}_{3} \mathrm{H}_{6}\right]^{+}\right)$

HRMS (EI): $\mathrm{m} / \mathrm{z}$ [M] ${ }^{+}$calcd for $\mathrm{C}_{14} \mathrm{H}_{15} \mathrm{NO}_{2}:$ :229.1103; found: 229.1107

\section{exo-3q}

Yield: $192 \mathrm{mg}$ (0.84 mmol at $1.99 \mathrm{mmol}$ scale, 42\%)

white solid, $50.5-51.5^{\circ} \mathrm{C}$

eluent: cyclohexane/toluene $20: 1$ to $3: 1$

$\mathrm{R}_{\mathrm{f}}=0.47$ (cyclohexane/toluene $3: 1$ )

${ }^{1} \mathrm{H}$ NMR $\left(400 \mathrm{MHz}, \mathrm{CDCl}_{3}\right): \delta=7.41-7.37(\mathrm{~m}, 1 \mathrm{H}), 7.30-7.18(\mathrm{~m}, 3 \mathrm{H})$, $6.99(\mathrm{dd}, J=10.1,1.4 \mathrm{~Hz}, 1 \mathrm{H}), 6.43(\mathrm{~d}, J=10.1 \mathrm{~Hz}, 1 \mathrm{H}), 3.62(\mathrm{dd}, J=7.6$, $1.3 \mathrm{~Hz}, 1 \mathrm{H}), 1.92-1.79(\mathrm{~m}, 1 \mathrm{H}), 1.12(\mathrm{~d}, J=6.8 \mathrm{~Hz}, 3 \mathrm{H}), 0.93(\mathrm{~d}, J=6.6 \mathrm{~Hz}$, $3 \mathrm{H}), 0.65(\mathrm{dd}, J=10.2,7.6 \mathrm{~Hz}, 1 \mathrm{H})$.

${ }^{13} \mathrm{C}$ NMR (100 MHz, $\left.\mathrm{CDCl}_{3}\right): \delta=132.4,128.8,128.4,128.3,127.7,127.1$, $125.0,121.5,69.4,40.0,35.1,27.0,21.7,21.3$.

MS (EI): $m / z(\%)=229\left(9,[M]^{+}\right), 186\left(50,\left[M-C_{3} \mathrm{H}_{7}\right]^{+}\right), 183\left(50,\left[M-N_{2}\right]^{+}\right)$, $173\left(71,\left[\mathrm{M}-\mathrm{C}_{4} \mathrm{H}_{8}\right]^{+}\right), 141\left(100,\left[\mathrm{M}-\mathrm{NO}_{2}-\mathrm{C}_{3} \mathrm{H}_{6}\right]^{+}\right)$

HRMS (EI): $\mathrm{m} / \mathrm{z}$ [M] ${ }^{+}$calcd for $\mathrm{C}_{14} \mathrm{H}_{15} \mathrm{NO}_{2}:$ :229.1103; found: 229.1104 

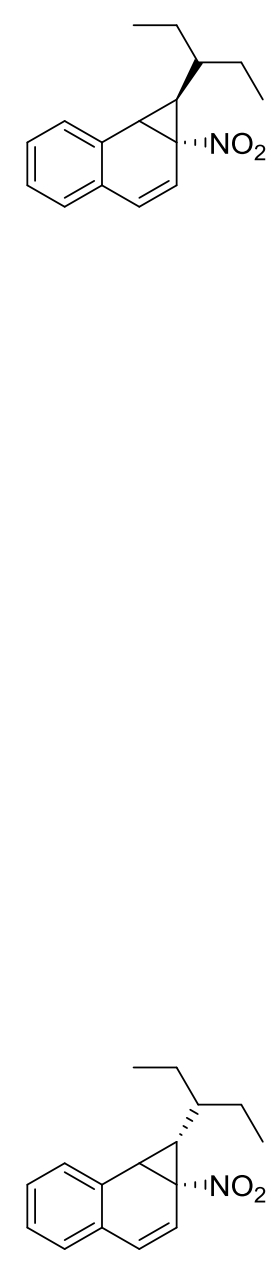

endo-3r

Yield: $261 \mathrm{mg}$ (1.02 $\mathrm{mmol}$ at $2.02 \mathrm{mmol}$ scale, 50\%)

white solid, $59.0-61.0^{\circ} \mathrm{C}$

eluent: cyclohexane/toluene $20: 1$ to $3: 1$

$\mathrm{R}_{\mathrm{f}}=0.45$ (cyclohexane/toluene $3: 1$ )

${ }^{1}$ H NMR (400 MHz, $\left.\mathrm{CDCl}_{3}\right): \delta=7.39-7.36(\mathrm{~m}, 1 \mathrm{H}), 7.31-7.20(\mathrm{~m}, 3 \mathrm{H})$, $6.76(\mathrm{dd}, J=10.1,1.2 \mathrm{~Hz}, 1 \mathrm{H}), 6.61(\mathrm{~d}, J=10.1 \mathrm{~Hz}, 1 \mathrm{H}), 3.77(\mathrm{dd}, J=11.3$, $0.7 \mathrm{~Hz}, 1 \mathrm{H}), 2.40(\mathrm{t}, J=11.1 \mathrm{~Hz}, 1 \mathrm{H}), 1.37-1.17(\mathrm{~m}, 2 \mathrm{H}), 1.14-0.95(\mathrm{~m}$, $2 \mathrm{H}), 0.76(\mathrm{t}, J=7.5 \mathrm{~Hz}, 3 \mathrm{H}), 0.57(\mathrm{t}, J=7.5 \mathrm{~Hz}, 3 \mathrm{H}), 0.49-0.37(\mathrm{~m}, 1 \mathrm{H})$.

${ }^{13} \mathrm{C}$ NMR (100 MHz, $\left.\mathrm{CDCl}_{3}\right): \delta=130.8,129.4,129.1,128.2,127.9,127.2$, $126.9,116.6,67.4,36.8,33.7,29.5,25.2,24.4,10.8,10.7$.

MS (EI): $m / z(\%)=257\left(5,[M]^{+}\right), 211\left(13,\left[M-N_{2}\right]^{+}\right), 173$ (29, $\left.\left[\mathrm{M}-\mathrm{NO}_{2}-\mathrm{C}_{6} \mathrm{H}_{12}\right]^{+}\right), 141\left(100,\left[\mathrm{M}-\mathrm{NO}_{2}-\mathrm{C}_{5} \mathrm{H}_{10}\right]^{+}\right), 127\left(29,\left[\mathrm{M}-\mathrm{NO}_{2}-\mathrm{C}_{6} \mathrm{H}_{12}\right]^{+}\right)$

HRMS (EI): $\mathrm{m} / \mathrm{z}$ [M] ${ }^{+}$calcd for $\mathrm{C}_{16} \mathrm{H}_{19} \mathrm{NO}_{2}$ : 257.1416; found: 257.1407

exo-3r

Yield: $213 \mathrm{mg}(0.83 \mathrm{mmol}$ at $2.02 \mathrm{mmol}$ scale, 41\%)

colorless oil

eluent: cyclohexane/toluene $20: 1$ to $3: 1$

$\mathrm{R}_{\mathrm{f}}=0.49$ (cyclohexane/toluene $3: 1$ )

${ }^{1}{ }_{H}$ NMR (400 MHz, $\left.\mathrm{CDCl}_{3}\right): \delta=7.40-7.36(\mathrm{~m}, 1 \mathrm{H}), 7.31-7.18(\mathrm{~m}, 3 \mathrm{H})$, $7.02(\mathrm{dd}, J=10.1,1.4 \mathrm{~Hz}, 1 \mathrm{H}), 6.42(\mathrm{~d}, J=10.1 \mathrm{~Hz}, 1 \mathrm{H}), 3.67(\mathrm{dd}, J=8.0$, $1.4 \mathrm{~Hz}, 1 \mathrm{H}), 1.68-1.54(\mathrm{~m}, 2 \mathrm{H}), 1.53-1.20(\mathrm{~m}, 3 \mathrm{H}), 1.01(\mathrm{t}, J=7.3 \mathrm{~Hz}$, $3 \mathrm{H}), 0.82(\mathrm{t}, J=7.5 \mathrm{~Hz}, 3 \mathrm{H}), 0.71(\mathrm{dd}, J=9.7,8.0 \mathrm{~Hz}, 1 \mathrm{H})$.

${ }^{13} \mathrm{C}$ NMR (100 MHz, $\left.\mathrm{CDCl}_{3}\right): \delta=132.3,128.9,128.4,128.3,127.7,127.1$, $125.0,121.4,68.2,38.8,38.4,35.4,26.6,26.3,11.6,10.6$.

MS (EI): m/z (\%) = $257\left(5,[\mathrm{M}]^{+}\right), 211\left(7,\left[\mathrm{M}-\mathrm{NO}_{2}\right]^{+}\right), 173\left(69,\left[\mathrm{M}-\mathrm{NO}_{2}-\right.\right.$ $\left.\left.\mathrm{C}_{6} \mathrm{H}_{12}\right]^{+}\right), 141\left(100,\left[\mathrm{M}-\mathrm{NO}_{2}-\mathrm{C}_{5} \mathrm{H}_{10}\right]^{+}\right), 127\left(50,\left[\mathrm{M}-\mathrm{NO}_{2}-\mathrm{C}_{6} \mathrm{H}_{12}\right]^{+}\right)$

HRMS (EI): $\mathrm{m} / \mathrm{z}[\mathrm{M}]^{+}$calcd for $\mathrm{C}_{16} \mathrm{H}_{19} \mathrm{NO}_{2}$ : 257.1416; found: 257.1428 

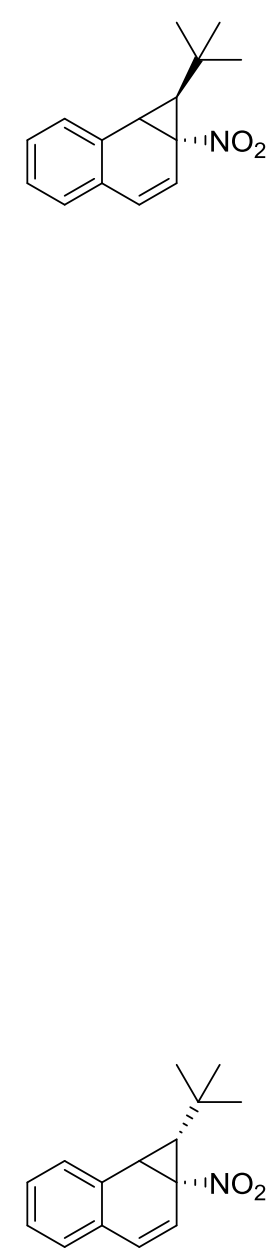

endo-3s

Yield: $227 \mathrm{mg}$ (0.93 mmol at $2.01 \mathrm{mmol}$ scale, 46\%)

white solid, $\mathrm{mp} 79.5-80.5^{\circ} \mathrm{C}$

eluent: cyclohexane/toluene $20: 1$ to $3: 1$

$\mathrm{R}_{\mathrm{f}}=0.39$ (cyclohexane/toluene $3: 1$ )

${ }^{1} \mathbf{H}$ NMR (400 MHz, $\left.\mathrm{CDCl}_{3}\right): \delta=7.44-7.40(\mathrm{~m}, 1 \mathrm{H}), 7.31-7.22(\mathrm{~m}, 2 \mathrm{H})$, $7.20-7.16(\mathrm{~m}, 1 \mathrm{H}), 6.84(\mathrm{dd}, J=10.0,1.2 \mathrm{~Hz}, 1 \mathrm{H}), 6.61(\mathrm{~d}, J=10.0 \mathrm{~Hz}, 1 \mathrm{H})$, $3.70(\mathrm{~d}, J=12.6 \mathrm{~Hz}, 1 \mathrm{H}), 2.26(\mathrm{~d}, J=12.6 \mathrm{~Hz}, 1 \mathrm{H}), 0.77(\mathrm{~s}, 9 \mathrm{H})$.

${ }^{13} \mathrm{C}$ NMR (100 MHz, $\left.\mathrm{CDCl}_{3}\right): \delta=131.6,130.2,129.3,128.9,128.5,128.0$, $127.4,116.9,67.2,35.7,33.1,32.7,28.9$.

MS (EI): $m / z(\%)=243\left(1,[M]^{+}\right), 197\left(49,\left[M-N_{2}\right]^{+}\right), 187\left(81,\left[M-C_{4} \mathrm{H}_{8}\right]^{+}\right)$, $173\left(26,\left[\mathrm{M}-\mathrm{C}_{5} \mathrm{H}_{10}\right]^{+}\right), 155\left(80,\left[\mathrm{M}-\mathrm{NO}_{2}-\mathrm{C}_{3} \mathrm{H}_{6}\right]^{+}\right), 141\left(100,\left[\mathrm{M}-\mathrm{NO}_{2}-\mathrm{C}_{4} \mathrm{H}_{8}\right]^{+}\right)$, $127\left(62,\left[\mathrm{M}-\mathrm{NO}_{2}-\mathrm{C}_{5} \mathrm{H}_{10}\right]^{+}\right)$

HRMS (EI): $\mathrm{m} / \mathrm{z}[\mathrm{M}]^{+}$calcd for $\mathrm{C}_{15} \mathrm{H}_{17} \mathrm{NO}_{2}$ : 243.1259; found: 243.1260

exo-3s

Yield: $51 \mathrm{mg}(0.21 \mathrm{mmol}$ at $2.01 \mathrm{mmol}$ scale, $10 \%)$

off-white solid, $\mathrm{mp} 52.0-53.5^{\circ} \mathrm{C}$

eluent: cyclohexane/toluene $20: 1$ to $3: 1$

$\mathrm{R}_{\mathrm{f}}=0.45$ (cyclohexane/toluene $3: 1$ )

${ }^{1} \mathbf{H}$ NMR (400 MHz, $\left.\mathrm{CDCl}_{3}\right): \delta=7.41-7.36(\mathrm{~m}, 1 \mathrm{H}), 7.30-7.15(\mathrm{~m}, 3 \mathrm{H})$, $6.75(\mathrm{dd}, J=9.9,1.4 \mathrm{~Hz}, 1 \mathrm{H}), 6.39(\mathrm{~d}, J=9.9 \mathrm{~Hz}, 1 \mathrm{H}), 3.65(\mathrm{dd}, J=8.3,1.4$ $\mathrm{Hz}, 1 \mathrm{H}), 1.02(\mathrm{~s}, 9 \mathrm{H}), 0.77(\mathrm{~d}, J=8.3 \mathrm{~Hz}, 1 \mathrm{H})$.

${ }^{13} \mathrm{C}$ NMR (100 MHz, $\left.\mathrm{CDCl}_{3}\right): \delta=132.9,128.8,128.51,128.46,127.8,127.0$, $125.6,123.3,70.8,42.4,31.2,29.5,28.4$.

MS (EI): $\mathrm{m} / \mathrm{z}(\%)=243\left(2,[\mathrm{M}]^{+}\right), 197\left(4,\left[\mathrm{M}-\mathrm{NO}_{2}\right]^{+}\right), 173\left(41,\left[\mathrm{M}-\mathrm{C}_{5} \mathrm{H}_{10}\right]^{+}\right)$, $141\left(39,\left[\mathrm{M}-\mathrm{NO}_{2}-\mathrm{C}_{4} \mathrm{H}_{8}\right]^{+}\right), 127\left(57,\left[\mathrm{M}-\mathrm{NO}_{2}-\mathrm{C}_{5} \mathrm{H}_{10}\right]^{+}\right)$

HRMS (EI): $\mathrm{m} / \mathrm{z}$ [M] ${ }^{+}$calcd for $\mathrm{C}_{15} \mathrm{H}_{17} \mathrm{NO}_{2}:$ :243.1259; found: 243.1269 


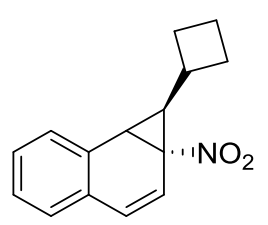

endo-3t

Yield: $361 \mathrm{mg}$ (1.50 mmol at $2.02 \mathrm{mmol}$ scale, 74\%)

off-white solid, $\mathrm{mp} 84.0-86.0^{\circ} \mathrm{C}$

eluent: cyclohexane/toluene $20: 1$ to $3: 1$

$\mathrm{R}_{\mathrm{f}}=0.37$ (cyclohexane/toluene $3: 1$ )

${ }^{1} \mathbf{H}$ NMR (400 MHz, $\left.\mathrm{CDCl}_{3}\right): \delta=7.36-7.31(\mathrm{~m}, 1 \mathrm{H}), 7.29-7.21(\mathrm{~m}, 3 \mathrm{H})$, $6.69(\mathrm{dd}, J=10.1,1.2 \mathrm{~Hz}, 1 \mathrm{H}), 6.61(\mathrm{dd}, J=10.1,0.5 \mathrm{~Hz}, 1 \mathrm{H}), 3.75(\mathrm{dd}, J=$ $11.1,1.1 \mathrm{~Hz}, 1 \mathrm{H}), 2.70(\mathrm{dd}, J=11.1,9.6 \mathrm{~Hz}, 1 \mathrm{H}), 1.99-1.88(\mathrm{~m}, 1 \mathrm{H}), 1.81-$ $1.56(\mathrm{~m}, 4 \mathrm{H}), 1.55-1.39(\mathrm{~m}, 2 \mathrm{H})$.

${ }^{13} \mathrm{C}$ NMR (100 MHz, $\left.\mathrm{CDCl}_{3}\right): \delta=130.8,129.1,128.7,128.3,127.9,127.2$, $127.1,116.2,67.6,36.9,30.4,29.0,27.5,27.2,18.4$.

MS (EI): $\mathrm{m} / \mathrm{z}(\%)=241\left(1,[\mathrm{M}]^{+}\right), 195\left(18,\left[\mathrm{M}-\mathrm{NO}_{2}\right]^{+}\right), 173\left(78,\left[\mathrm{M}-\mathrm{C}_{5} \mathrm{H}_{8}\right]^{+}\right)$, $141\left(100,\left[\mathrm{M}-\mathrm{NO}_{2}-\mathrm{C}_{4} \mathrm{H}_{6}\right]^{+}\right), 127\left(79,\left[\mathrm{M}-\mathrm{NO}_{2}-\mathrm{C}_{5} \mathrm{H}_{8}\right]^{+}\right)$

HRMS (EI): $\mathrm{m} / \mathrm{z}[\mathrm{M}]^{+}$calcd for $\mathrm{C}_{15} \mathrm{H}_{15} \mathrm{NO}_{2}: 241.1103$; found: 241.1106

Exo-3t was inseparable from other impurities (mainly alkylated product), therefore it was placed in $25 \mathrm{~mL}$ round-bottom flask, dissolved in toluene $(10 \mathrm{~mL})$ and heated to $90^{\circ} \mathrm{C}$. After $24 \mathrm{~h}$ reaction mixture was separated with column chromatography yielding endo-3t. 

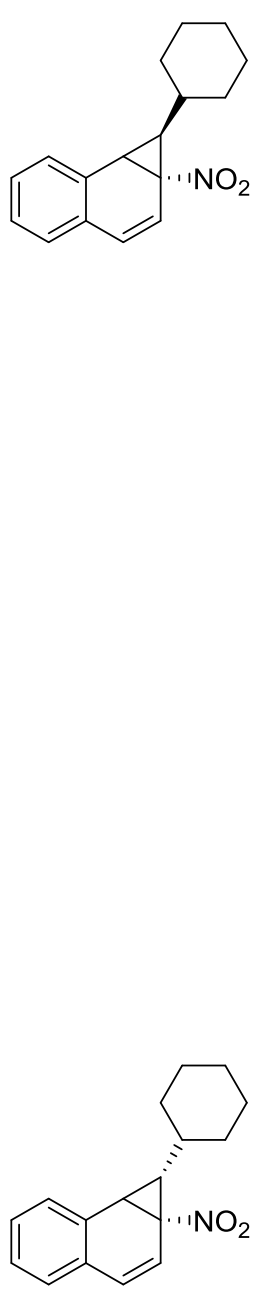

endo-3u

Yield: $299 \mathrm{mg}$ (1.11 $\mathrm{mmol}$ at $2.04 \mathrm{mmol}$ scale, 54\%)

white solid, $\mathrm{mp} 82.5-83.5^{\circ} \mathrm{C}$

eluent: cyclohexane/toluene $20: 1$ to $3: 1$

$\mathrm{R}_{\mathrm{f}}=0.40$ (cyclohexane/toluene $3: 1$ )

${ }^{1}$ H NMR (400 MHz, $\left.\mathrm{CDCl}_{3}\right): \delta=7.39-7.33(\mathrm{~m}, 1 \mathrm{H}), 7.30-7.21(\mathrm{~m}, 3 \mathrm{H})$, $6.76(\mathrm{dd}, J=10.1,1.1 \mathrm{~Hz}, 1 \mathrm{H}), 6.61(\mathrm{dd}, J=10.1,0.5 \mathrm{~Hz}, 1 \mathrm{H}), 3.73(\mathrm{dd}, J=$ $11.2,0.8 \mathrm{~Hz}, 1 \mathrm{H}), 2.35(\mathrm{t}, J=11.0 \mathrm{~Hz}, 1 \mathrm{H}), 1.63-1.42(\mathrm{~m}, 4 \mathrm{H}), 1.24-1.14$ $(m, 1 H), 1.11-0.89(m, 4 H), 0.82-0.68(m, 1 H), 0.46-0.33(m, 1 H)$.

${ }^{13} \mathrm{C}$ NMR (100 MHz, $\left.\mathrm{CDCl}_{3}\right): \delta=130.8,129.0,128.8,128.3,128.0,127.2$, $127.1,116.0,67.0,36.4,31.3,31.2,30.7,30.5,25.8,25.51,25.50$.

MS (EI): $m / z(\%)=269\left(4,[M]^{+}\right), 223\left(11,\left[M-N_{2}\right]^{+}\right), 187\left(26,\left[M-C_{6} \mathrm{H}_{10}\right]^{+}\right)$, $141\left(100,\left[\mathrm{M}-\mathrm{NO}_{2}-\mathrm{C}_{6} \mathrm{H}_{10}\right]^{+}\right), 127\left(28,\left[\mathrm{M}-\mathrm{NO}_{2}-\mathrm{C}_{7} \mathrm{H}_{12}\right]^{+}\right)$

HRMS (EI): $\mathrm{m} / \mathrm{z}[\mathrm{M}]^{+}$calcd for $\mathrm{C}_{17} \mathrm{H}_{19} \mathrm{NO}_{2}:$ 269.1416; found: 269.1412

\section{exo-3u}

Yield: $246 \mathrm{mg}(0.92 \mathrm{mmol}$ at $2.04 \mathrm{mmol}$ scale, 45\%)

off-white solid, $\mathrm{mp} 89.0-91.5^{\circ} \mathrm{C}$

eluent: cyclohexane/toluene $20: 1$ to $3: 1$

$\mathrm{R}_{\mathrm{f}}=0.50$ (cyclohexane/toluene $3: 1$ )

${ }^{1}{ }_{H}$ NMR (400 MHz, $\left.\mathrm{CDCl}_{3}\right): \delta=7.40-7.34(\mathrm{~m}, 1 \mathrm{H}), 7.30-7.17(\mathrm{~m}, 3 \mathrm{H})$, 6.99 (dd, $J=10.1,1.4 \mathrm{~Hz}, 1 \mathrm{H}), 6.41(\mathrm{~d}, J=10.1 \mathrm{~Hz}, 1 \mathrm{H}), 3.62(\mathrm{dd}, J=7.6$, $1.3 \mathrm{~Hz}, 1 \mathrm{H}), 2.00-1.90(\mathrm{~m}, 1 \mathrm{H}), 1.80-1.71(\mathrm{~m}, 1 \mathrm{H}), 1.69-1.48(\mathrm{~m}, 4 \mathrm{H})$, $1.39-0.95(\mathrm{~m}, 5 \mathrm{H}), 0.69(\mathrm{dd}, J=9.9,7.6 \mathrm{~Hz}, 1 \mathrm{H})$.

${ }^{13} \mathrm{C}$ NMR (100 MHz, $\left.\mathrm{CDCl}_{3}\right): \delta=132.4,128.8,128.30,128.27,127.6,127.0$, $124.9,121.5,68.9,38.5,35.8,34.7,32.0,31.9,25.9,25.8,25.5$.

MS (EI): $\mathrm{m} / \mathrm{z}(\%)=269\left(6,[\mathrm{M}]^{+}\right), 223\left(7,\left[\mathrm{M}-\mathrm{NO}_{2}\right]^{+}\right), 173\left(37,\left[\mathrm{M}-\mathrm{C}_{7} \mathrm{H}_{12}\right]^{+}\right)$, $141\left(100,\left[\mathrm{M}-\mathrm{NO}_{2}-\mathrm{C}_{6} \mathrm{H}_{10}\right]^{+}\right), 127\left(34,\left[\mathrm{M}-\mathrm{NO}_{2}-\mathrm{C}_{7} \mathrm{H}_{12}\right]^{+}\right)$

HRMS (EI): $\mathrm{m} / \mathrm{z}$ [M] ${ }^{+}$calcd for $\mathrm{C}_{17} \mathrm{H}_{19} \mathrm{NO}_{2}$ : 269.1416; found: 269.1425 


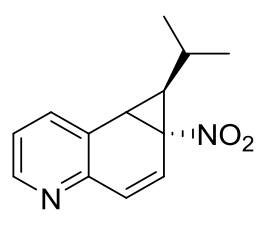

endo-3v

Yield: $303 \mathrm{mg}(1.32 \mathrm{mmol}$ at $2.00 \mathrm{mmol}$ scale, $66 \%)$

off-white solid, $\mathrm{mp} 118.5-121.0^{\circ} \mathrm{C}$

eluent: cyclohexane/toluene $20: 1$ to $3: 1$

$\mathrm{R}_{\mathrm{f}}=0.25$ (cyclohexane/ethyl acetate $3: 1$ )

${ }^{1} \mathrm{H}$ NMR (400 MHz, $\left.\mathrm{CDCl}_{3}\right): \delta=8.53(\mathrm{dd}, J=4.8,1.6 \mathrm{~Hz}, 1 \mathrm{H}), 7.72(\mathrm{dd}, J$ $=7.7,1.6 \mathrm{~Hz}, 1 \mathrm{H}), 7.22(\mathrm{dd}, J=7.7,4.8 \mathrm{~Hz}, 1 \mathrm{H}), 7.08(\mathrm{dd}, J=10.4,1.3 \mathrm{~Hz}$, $1 \mathrm{H}), 6.88(\mathrm{~d}, J=10.4 \mathrm{~Hz}, 1 \mathrm{H}), 3.76(\mathrm{dd}, J=11.2,1.3 \mathrm{~Hz}, 1 \mathrm{H}), 2.41(\mathrm{dd}, J=$ $11.2,10.2 \mathrm{~Hz}, 1 \mathrm{H}), 0.85(\mathrm{~d}, J=6.2 \mathrm{~Hz}, 3 \mathrm{H}), 0.78-0.67(\mathrm{~m}, 1 \mathrm{H}), 0.66(\mathrm{~d}, J=$ $6.0 \mathrm{~Hz}, 3 \mathrm{H})$.

${ }^{13} \mathrm{C}$ NMR (100 MHz, $\left.\mathrm{CDCl}_{3}\right): \delta=149.7,148.5,136.5,128.9,125.0,122.7$, 120.7, 67.0, 35.9, 31.8, 22.1, 20.7, 20.0.

MS (EI): m/z (\%) = $230\left(6,[\mathrm{M}]^{+}\right), 184\left(66,\left[\mathrm{M}-\mathrm{NO}_{2}\right]^{+}\right), 142(100$, $\left.\left[\mathrm{M}-\mathrm{NO}_{2}-\mathrm{C}_{3} \mathrm{H}_{6}\right]^{+}\right), 128\left(38,\left[\mathrm{M}-\mathrm{NO}_{2}-\mathrm{C}_{4} \mathrm{H}_{8}\right]^{+}\right)$

HRMS (EI): $\mathrm{m} / \mathrm{z}[\mathrm{M}]^{+}$calcd for $\mathrm{C}_{13} \mathrm{H}_{14} \mathrm{~N}_{2} \mathrm{O}_{2}: 230.1055$; found: 230.1058

Exo-3v isomerized into endo-3v during attempts of recrystallization from dichloromethane/heptane (crystals of endo-3v were formed). 


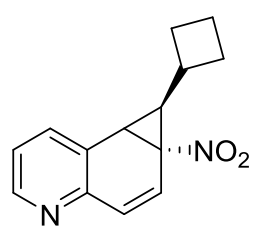

endo-3w

Yield: $261 \mathrm{mg}$ (1.08 mmol at $2.02 \mathrm{mmol}$ scale, 53\%)

pale yellow solid, $\mathrm{mp} 100.0-102.5^{\circ} \mathrm{C}$

eluent: toluene/ethyl acetate $10: 1$ to $2: 1$

$\mathrm{R}_{\mathrm{f}}=0.27$ (cyclohexane/ethyl acetate 3:1)

${ }^{1} \mathrm{H}$ NMR (400 MHz, $\left.\mathrm{CDCl}_{3}\right): \delta=8.45(\mathrm{dd}, J=4.8,1.7 \mathrm{~Hz}, 1 \mathrm{H}), 7.60(\mathrm{dd}, J$ $=7.7,1.7 \mathrm{~Hz}, 1 \mathrm{H}), 7.13(\mathrm{dd}, J=7.7,4.8 \mathrm{~Hz}, 1 \mathrm{H}), 6.92(\mathrm{dd}, J=10.3,1.4 \mathrm{~Hz}$, $1 \mathrm{H}), 6.79(\mathrm{dd}, J=10.3,0.7 \mathrm{~Hz}, 1 \mathrm{H}), 3.69(\mathrm{dd}, J=11.1,1.4 \mathrm{~Hz}, 1 \mathrm{H}), 2.71$ (dd, $J=11.1,9.6 \mathrm{~Hz}, 1 \mathrm{H}), 1.98-1.81(\mathrm{~m}, 1 \mathrm{H}), 1.75-1.53(\mathrm{~m}, 4 \mathrm{H}), 1.49-1.32$ $(\mathrm{m}, 2 \mathrm{H})$.

${ }^{13} \mathrm{C}$ NMR (100 MHz, $\left.\mathrm{CDCl}_{3}\right): \delta=149.6,148.4,136.2,128.7,125.0,122.5$, $120.7,67.0,35.9,29.6,28.5,27.3,27.0,18.2$.

HRMS (ESI): $\mathrm{m} / \mathrm{z}[\mathrm{M}+\mathrm{H}]^{+}$calcd for $\mathrm{C}_{14} \mathrm{H}_{15} \mathrm{~N}_{2} \mathrm{O}_{2}: 243.1134$, found: 243.1127

Exo-3w was inseparable from other impurities (mainly endo isomer), therefore it was placed in $25 \mathrm{~mL}$ round-bottom flask, dissolved in toluene $(10 \mathrm{~mL})$ and heated to $90^{\circ} \mathrm{C}$. After $24 \mathrm{~h}$ reaction mixture was separated with column chromatography yielding endo-3w. 


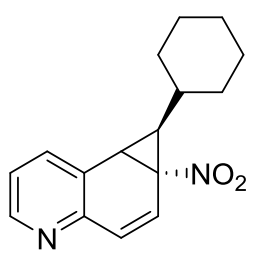

endo-3x

Yield: $408 \mathrm{mg}$ (1.51 $\mathrm{mmol}$ at $2.02 \mathrm{mmol}$ scale, 75\%)

brown solid, $\mathrm{mp} 86.5-88.0^{\circ} \mathrm{C}$

eluent: toluene/ethyl acetate $50: 1$ to $3: 1$

$\mathrm{R}_{\mathrm{f}}=0.30$ (cyclohexane/ethyl acetate $3: 1$ )

${ }^{1} \mathrm{H}$ NMR (400 MHz, $\left.\mathrm{CDCl}_{3}\right): \delta=8.44(\mathrm{dd}, J=4.8,1.7 \mathrm{~Hz}, 1 \mathrm{H}), 7.63(\mathrm{dd}, J$ $=7.7,1.7 \mathrm{~Hz}, 1 \mathrm{H}), 7.13(\mathrm{dd}, J=7.7,4.8 \mathrm{~Hz}, 1 \mathrm{H}), 6.98(\mathrm{dd}, J=10.3,1.3 \mathrm{~Hz}$, 1H), $6.77(\mathrm{dd}, J=10.3,0.7 \mathrm{~Hz}, 1 \mathrm{H}), 3.66(\mathrm{dd}, J=11.1,1.3 \mathrm{~Hz}, 1 \mathrm{H}), 2.33(\mathrm{t}, J$ $=11.1 \mathrm{~Hz}, 1 \mathrm{H}), 1.55-1.47(\mathrm{~m}, 1 \mathrm{H}), 1.45-1.33(\mathrm{~m}, 3 \mathrm{H}), 1.12-1.04(\mathrm{~m}$, $1 \mathrm{H}), 1.02-0.82(\mathrm{~m}, 4 \mathrm{H}), 0.76-0.60(\mathrm{~m}, 1 \mathrm{H}), 0.41-0.17(\mathrm{~m}, 1 \mathrm{H})$.

${ }^{13} \mathrm{C}$ NMR (100 MHz, $\left.\mathrm{CDCl}_{3}\right): \delta=149.5,148.3,136.3,128.6,124.8,122.5$, $120.5,66.3,35.4,30.8,30.7,30.2,30.1,25.5,25.23,25.17$.

HRMS (ESI): $\mathrm{m} / \mathrm{z}[\mathrm{M}+\mathrm{H}]^{+}$calcd for $\mathrm{C}_{16} \mathrm{H}_{19} \mathrm{~N}_{2} \mathrm{O}_{2}: 271.1447$, found: 271.1440<smiles>O=[N+]([O-])C12C=Cc3ncccc3C1C2C1CCCCC1</smiles>

exo-3x

Yield: $45 \mathrm{mg}$ (0.17 $\mathrm{mmol}$ at $2.02 \mathrm{mmol}$ scale, $8 \%)$

orange solid, $\mathrm{mp} 88.0^{\circ} \mathrm{C}$ (dec.)

eluent: toluene/ethyl acetate $50: 1$ to $3: 1$

$\mathrm{R}_{\mathrm{f}}=0.24$ (cyclohexane/ethyl acetate $3: 1$ )

${ }^{1} \mathrm{H}$ NMR $\left(400 \mathrm{MHz}, \mathrm{CDCl}_{3}\right): \delta=8.46(\mathrm{~d}, J=4.9 \mathrm{~Hz}, 1 \mathrm{H}), 7.68(\mathrm{dd}, J=7.7$, $1.6 \mathrm{~Hz}, 1 \mathrm{H}), 7.29(\mathrm{dd}, J=10.3,1.6 \mathrm{~Hz}, 1 \mathrm{H}), 7.18(\mathrm{dd}, J=7.7,4.9 \mathrm{~Hz}, 1 \mathrm{H})$, $6.67(\mathrm{dd}, J=10.3,0.7 \mathrm{~Hz}, 1 \mathrm{H}), 3.61(\mathrm{dd}, J=7.5,1.6 \mathrm{~Hz}, 1 \mathrm{H}), 1.95-1.83(\mathrm{~m}$, $1 \mathrm{H}), 1.77-1.68(\mathrm{~m}, 1 \mathrm{H}), 1.67-1.42(\mathrm{~m}, 4 \mathrm{H}), 1.38-0.94(\mathrm{~m}, 5 \mathrm{H}), 0.70(\mathrm{dd}$, $J=9.9,7.6 \mathrm{~Hz}, 1 \mathrm{H})$.

${ }^{13} \mathrm{C}$ NMR $\left(100 \mathrm{MHz}, \mathrm{CDCl}_{3}\right): \delta=147.9,147.7,135.5,128.4,126.8,126.3$, $122.7,68.4,38.4,35.8,33.7,31.90,31.86,25.9,25.7,25.4$.

HRMS (ESI): $\mathrm{m} / \mathrm{z}[\mathrm{M}+\mathrm{H}]^{+}$calcd for $\mathrm{C}_{16} \mathrm{H}_{19} \mathrm{~N}_{2} \mathrm{O}_{2}: 271.1447$, found: 271.1438 


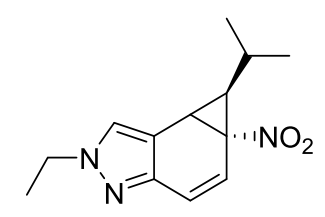

\section{endo-3y}

Yield: $405 \mathrm{mg}$ (1.64 mmol at $2.03 \mathrm{mmol}$ scale, 81\%)

pale yellow oil

eluent: toluene/ethyl acetate $20: 1$ to $6: 1$

$\mathrm{R}_{\mathrm{f}}=0.25$ (cyclohexane/ethyl acetate $3: 1$ )

${ }^{1} \mathrm{H}$ NMR (400 MHz, CDCl $)$ ): $\delta=7.31(\mathrm{~s}, 1 \mathrm{H}), 6.69(\mathrm{dd}, J=10.3,0.7 \mathrm{~Hz}, 1 \mathrm{H})$, $6.67(\mathrm{dd}, J=10.3,0.8 \mathrm{~Hz}, 1 \mathrm{H}), 4.09(\mathrm{qd}, J=7.3,1.0 \mathrm{~Hz}, 2 \mathrm{H}), 3.61(\mathrm{~d}$, $J=10.6 \mathrm{~Hz}, 1 \mathrm{H}), 2.18(\mathrm{dd}, J=10.6,9.9 \mathrm{~Hz}, 1 \mathrm{H}), 1.40(\mathrm{t}, J=7.3 \mathrm{~Hz}, 3 \mathrm{H}), 0.79$ $(\mathrm{d}, J=5.5 \mathrm{~Hz}, 3 \mathrm{H}), 0.77-0.69(\mathrm{~m}, 1 \mathrm{H}), 0.68(\mathrm{~d}, J=5.0 \mathrm{~Hz}, 3 \mathrm{H})$.

${ }^{13} \mathrm{C}$ NMR $\left(100 \mathrm{MHz}, \mathrm{CDCl}_{3}\right): \delta=145.4,126.4,120.0,117.3,109.9,67.7$, $47.2,33.8,30.9,22.3,20.9,20.0,15.4$.

HRMS (ESI): $\mathrm{m} / \mathrm{z}[\mathrm{M}+\mathrm{H}]^{+}$calcd for $\mathrm{C}_{13} \mathrm{H}_{18} \mathrm{~N}_{3} \mathrm{O}_{2}: 248.1399$, found: 248.1393

Exo-3y was inseparable from other impurities (mainly endo isomer), therefore it was placed in $25 \mathrm{~mL}$ round-bottom flask, dissolved in toluene $(10 \mathrm{~mL})$ and heated to $90^{\circ} \mathrm{C}$. After $24 \mathrm{~h}$ reaction mixture was separated with column chromatography yielding endo-3y.

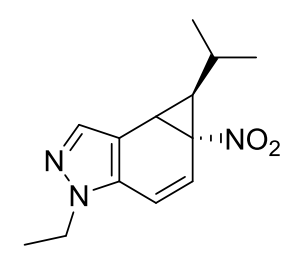

endo-3z

Yield: $295 \mathrm{mg}$ (1.19 $\mathrm{mmol}$ at $2.01 \mathrm{mmol}$ scale, 59\%)

off-white solid, $\mathrm{mp} 80.0-83.5^{\circ} \mathrm{C}$

eluent: toluene/ethyl acetate $20: 1$ to $5: 1$

$\mathrm{R}_{\mathrm{f}}=0.24$ (cyclohexane/ethyl acetate $3: 1$ )

${ }^{1} \mathrm{H}$ NMR $\left(400 \mathrm{MHz}, \mathrm{CDCl}_{3}\right): \delta=7.44(\mathrm{~d}, J=0.9 \mathrm{~Hz}, 1 \mathrm{H}), 6.76(\mathrm{dd}, J=10.4$, $0.9 \mathrm{~Hz}, 1 \mathrm{H}), 6.58(\mathrm{dd}, J=10.4,0.9 \mathrm{~Hz}, 1 \mathrm{H}), 4.16(\mathrm{q}, J=7.3 \mathrm{~Hz}, 2 \mathrm{H}), 3.72$ (dd, $J=10.7,0.9 \mathrm{~Hz}, 1 \mathrm{H}), 2.26(\mathrm{t}, J=10.7 \mathrm{~Hz}, 1 \mathrm{H}), 1.40(\mathrm{t}, J=7.3 \mathrm{~Hz}, 3 \mathrm{H})$, $0.81(\mathrm{~d}, J=6.6 \mathrm{~Hz}, 3 \mathrm{H}), 0.71(\mathrm{~d}, J=6.6 \mathrm{~Hz}, 3 \mathrm{H}), 0.62-0.48(\mathrm{~m}, 1 \mathrm{H})$.

${ }^{13} \mathrm{C}$ NMR (100 MHz, $\left.\mathrm{CDCl}_{3}\right): \delta=136.0,134.9,117.9,113.8,110.9,68.2$, $44.1,32.5,31.1,22.0,20.8,20.1,15.5$.

HRMS (ESI): $\mathrm{m} / \mathrm{z}[\mathrm{M}+\mathrm{H}]^{+}$calcd for $\mathrm{C}_{13} \mathrm{H}_{18} \mathrm{~N}_{3} \mathrm{O}_{2}: 248.1399$, found: 248.1392 


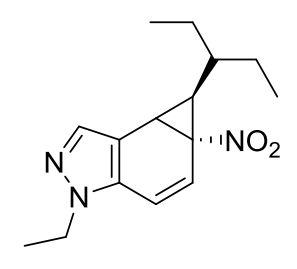

endo-3aa

Yield: $488 \mathrm{mg}$ (1.77 $\mathrm{mmol}$ at $2.00 \mathrm{mmol}$ scale, 89\%)

yellowish oil

eluent: toluene/ethyl acetate $20: 1$ to $5: 1$

$\mathrm{R}_{\mathrm{f}}=0.23$ (toluene/ethyl acetate $20: 1$ )

${ }^{1}$ H NMR (400 MHz, $\left.\mathrm{CDCl}_{3}\right): \delta=7.37(\mathrm{~d}, J=0.9 \mathrm{~Hz}, 1 \mathrm{H}), 6.69(\mathrm{dd}, J=10.4$, $0.9 \mathrm{~Hz}, 1 \mathrm{H}), 6.53(\mathrm{dd}, J=10.4,0.9 \mathrm{~Hz}, 1 \mathrm{H}), 4.11(\mathrm{q}, J=7.3 \mathrm{~Hz}, 2 \mathrm{H}), 3.68$ $(\mathrm{dd}, J=10.9,0.9 \mathrm{~Hz}, 1 \mathrm{H}), 2.25(\mathrm{t}, J=10.9 \mathrm{~Hz}, 1 \mathrm{H}), 1.32(\mathrm{t}, J=7.3 \mathrm{~Hz}, 3 \mathrm{H})$, $1.26-0.96(\mathrm{~m}, 4 \mathrm{H}), 0.67(\mathrm{t}, J=7.5 \mathrm{~Hz}, 3 \mathrm{H}), 0.58(\mathrm{t}, J=7.5 \mathrm{~Hz}, 3 \mathrm{H}), 0.29-$ $0.17(\mathrm{~m}, 1 \mathrm{H})$.

${ }^{13} \mathrm{C}$ NMR $\left(100 \mathrm{MHz}, \mathrm{CDCl}_{3}\right): \delta=136.0,134.6,118.3,113.4,111.2,67.9$, $43.9,33.3,32.1,27.9,24.9,24.2,15.4,10.63,10.59$.

HRMS (ESI): $\mathrm{m} / \mathrm{z}[\mathrm{M}+\mathrm{H}]^{+}$calcd for $\mathrm{C}_{15} \mathrm{H}_{22} \mathrm{~N}_{3} \mathrm{O}_{2}: 276.1712$, found: 276.1705

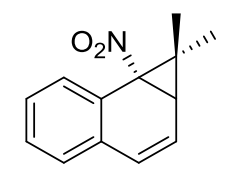

$3 a b$

Yield: $277 \mathrm{mg}$ (1.29 mmol at $1.53 \mathrm{mmol}$ scale, 84\%)

off-white crystals, $50.0-52.5^{\circ} \mathrm{C}$

eluent: cyclohexane/toluene $20: 1$ to $3: 1$

$R_{f}=0.40$ (cyclohexane/toluene $3: 1$ )

${ }^{1} \mathrm{H}$ NMR (400 MHz, $\left.\mathrm{CDCl}_{3}\right): \delta=7.90-7.84(\mathrm{~m}, 1 \mathrm{H}), 7.33-7.24(\mathrm{~m}, 2 \mathrm{H})$, $7.22-7.14(\mathrm{~m}, 1 \mathrm{H}), 6.51(\mathrm{~d}, J=9.7 \mathrm{~Hz}, 1 \mathrm{H}), 6.06(\mathrm{dd}, J=9.7,5.6 \mathrm{~Hz}, 1 \mathrm{H})$, $2.77(\mathrm{~d}, J=5.6 \mathrm{~Hz}, 1 \mathrm{H}), 1.39(\mathrm{~s}, 3 \mathrm{H}), 0.61(\mathrm{~s}, 3 \mathrm{H})$.

${ }^{13} \mathrm{C}$ NMR (100 MHz, $\left.\mathrm{CDCl}_{3}\right): \delta=131.7,128.5,128.2,128.0,127.0,126.0$, $125.3,122.4,76.2,35.7,22.7,17.6,14.5$.

MS (EI): $m / z(\%)=215\left(1,[M]^{+}\right), 169\left(100,\left[\mathrm{M}-\mathrm{NO}_{2}\right]^{+}\right), 141(81$, $\left[\mathrm{M}-\mathrm{NO}_{2}-\mathrm{C}_{2} \mathrm{H}_{4}\right]^{+}$)

HRMS (EI): $\mathrm{m} / \mathrm{z}$ [M] $]^{+}$calcd for $\mathrm{C}_{13} \mathrm{H}_{13} \mathrm{NO}_{2}$ : 215.0946; found: 215.0941 


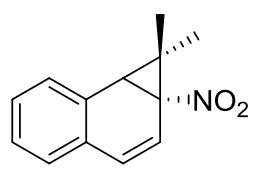

3ac

Yield: $372 \mathrm{mg}$ (1.73 mmol at $2.01 \mathrm{mmol}$ scale, 87\%)

off-white solid, $\mathrm{mp} 47.0-49.5^{\circ} \mathrm{C}$

eluent: cyclohexane/toluene $20: 1$ to $3: 1$

$\mathrm{R}_{\mathrm{f}}=0.47$ (cyclohexane/ethyl acetate 20:1)

${ }^{1} \mathbf{H}$ NMR (400 MHz, $\left.\mathrm{CDCl}_{3}\right): \delta=7.37-7.34(\mathrm{~m}, 1 \mathrm{H}), 7.30-7.19(\mathrm{~m}, 3 \mathrm{H})$, $6.72(\mathrm{dd}, J=10.0,1.3 \mathrm{~Hz}, 1 \mathrm{H}), 6.59(\mathrm{~d}, J=10.0 \mathrm{~Hz}, 1 \mathrm{H}), 3.61(\mathrm{~d}, J=1.3 \mathrm{~Hz}$, $1 \mathrm{H}), 1.45(\mathrm{~s}, 3 \mathrm{H}), 0.71(\mathrm{~s}, 3 \mathrm{H})$.

${ }^{13} \mathrm{C}$ NMR (100 MHz, $\left.\mathrm{CDCl}_{3}\right): \delta=130.5,130.0,129.2,128.3,128.1,128.0$, $127.1,118.8,75.2,38.1,21.3,20.0,15.2$.

MS (EI): m/z (\%) = $215\left(4,[M]^{+}\right), 169\left(60,\left[\mathrm{M}-\mathrm{NO}_{2}\right]^{+}\right), 141$ (70, $\left.\left[\mathrm{M}-\mathrm{NO}_{2}-\mathrm{C}_{2} \mathrm{H}_{4}\right]^{+}\right), 127\left(100,\left[\mathrm{M}-\mathrm{NO}_{2}-\mathrm{C}_{3} \mathrm{H}_{6}\right]^{+}\right)$

HRMS (EI): $\mathrm{m} / \mathrm{z}[\mathrm{M}]^{+}$calcd for $\mathrm{C}_{13} \mathrm{H}_{13} \mathrm{NO}_{2}:$ 215.0946; found: 215.0947

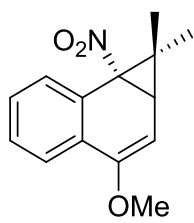

3ad

Yield: $479 \mathrm{mg}$ (1.95 mmol at $2.01 \mathrm{mmol}$ scale, 97\%)

yellow oil

eluent: cyclohexane/toluene 5:1 to $1: 1$

$\mathrm{R}_{\mathrm{f}}=0.30$ (cyclohexane/toluene $3: 1$ )

${ }^{1} \mathrm{H}$ NMR $\left(400 \mathrm{MHz}, \mathrm{CDCl}_{3}\right): \delta=7.91-7.86(\mathrm{~m}, 1 \mathrm{H}), 7.80-7.75(\mathrm{~m}, 1 \mathrm{H})$, $7.40-7.27(\mathrm{~m}, 2 \mathrm{H}), 5.09(\mathrm{~d}, J=6.1 \mathrm{~Hz}, 1 \mathrm{H}), 3.73(\mathrm{~s}, 3 \mathrm{H}), 2.78(\mathrm{~d}, J=6.1 \mathrm{~Hz}$, $1 \mathrm{H}), 1.36(\mathrm{~s}, 3 \mathrm{H}), 0.61(\mathrm{~s}, 3 \mathrm{H})$.

${ }^{13} \mathrm{C}$ NMR $\left(100 \mathrm{MHz}, \mathrm{CDCl}_{3}\right): \delta=151.2,129.4,128.28,128.26,126.8,126.0$, $122.7,90.9,75.1,54.8,33.8,22.6,19.0,14.5$.

MS (EI): m/z (\%) = $245\left(1,[\mathrm{M}]^{+}\right), 199\left(100,\left[\mathrm{M}-\mathrm{NO}_{2}\right]^{+}\right), 184$ (22, $\left.\left[\mathrm{M}-\mathrm{NO}_{2}-\mathrm{CH}_{3}\right]^{+}\right), 171\left(25,\left[\mathrm{M}-\mathrm{NO}_{2}-\mathrm{C}_{2} \mathrm{H}_{4}\right]^{+}\right)$

HRMS (EI): $\mathrm{m} / \mathrm{z}[\mathrm{M}]^{+}$calcd for $\mathrm{C}_{14} \mathrm{H}_{15} \mathrm{NO}_{3}: 245.1052$; found: 245.1059 


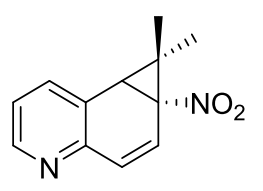

3ae

Yield: $258 \mathrm{mg}$ (1.20 mmol at $2.00 \mathrm{mmol}$ scale, 60\%)

pale yellow oil

eluent: toluene/ethyl acetate $6: 1$

$\mathrm{R}_{\mathrm{f}}=0.19$ (cyclohexane/ethyl acetate $3: 1$ )

${ }^{1} \mathrm{H}$ NMR (400 MHz, $\left.\mathrm{CDCl}_{3}\right): \delta=8.45(\mathrm{dd}, J=4.8,1.7 \mathrm{~Hz}, 1 \mathrm{H}), 7.63(\mathrm{dd}, J$ $=7.7,1.7 \mathrm{~Hz}, 1 \mathrm{H}), 7.16(\mathrm{dd}, J=7.7,4.8 \mathrm{~Hz}, 1 \mathrm{H}), 6.95(\mathrm{dd}, J=10.3,1.5 \mathrm{~Hz}$ $1 \mathrm{H}), 6.79(\mathrm{dd}, J=10.3,0.7 \mathrm{~Hz}, 1 \mathrm{H}), 3.55(\mathrm{~d}, J=1.5 \mathrm{~Hz}, 1 \mathrm{H}), 1.42(\mathrm{~s}, 3 \mathrm{H})$, $0.70(\mathrm{~s}, 3 \mathrm{H})$.

${ }^{13} \mathrm{C}$ NMR (100 MHz, $\left.\mathrm{CDCl}_{3}\right): \delta=149.5,148.3,136.6,129.8,125.8,123.5$, $122.6,74.6,37.4,21.2,19.9,15.1$.

HRMS (ESI): $\mathrm{m} / \mathrm{z}[\mathrm{M}+\mathrm{H}]^{+}$calcd for $\mathrm{C}_{12} \mathrm{H}_{13} \mathrm{~N}_{2} \mathrm{O}_{2}: 217.0977$, found: 217.0968

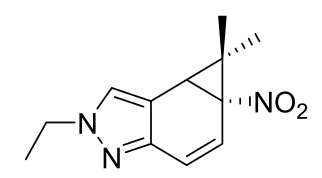

3af

Yield: $463 \mathrm{mg}$ (1.99 $\mathrm{mmol}$ at $2.00 \mathrm{mmol}$ scale, 99\%)

pale yellow solid, $\mathrm{mp} 64.5-67.5^{\circ} \mathrm{C}$

eluent: toluene/ethyl acetate $4: 1$ to $3: 1$

$\mathrm{R}_{\mathrm{f}}=0.22$ (cyclohexane/ethyl acetate $3: 1$ )

${ }^{1} \mathrm{H}$ NMR (400 MHz, $\left.\mathrm{CDCl}_{3}\right): \delta=7.27(\mathrm{~s}, 1 \mathrm{H}), 6.63(\mathrm{~d}, J=10.3 \mathrm{~Hz}, 1 \mathrm{H}), 6.58$ (d, $J=10.3 \mathrm{~Hz}, 1 \mathrm{H}), 4.05(\mathrm{q}, J=7.3 \mathrm{~Hz}, 2 \mathrm{H}), 3.45(\mathrm{~s}, 1 \mathrm{H}), 1.38(\mathrm{t}, J=7.3 \mathrm{~Hz}$, $3 \mathrm{H}), 1.27(\mathrm{~s}, 3 \mathrm{H}), 0.66(\mathrm{~s}, 3 \mathrm{H})$.

${ }^{13} \mathrm{C}$ NMR (100 MHz, $\left.\mathrm{CDCl}_{3}\right): \delta=145.2,126.8,121.1,120.0,110.9,75.4$, 47.0, 31.7, 22.3, 19.7, 15.33, 15.31 .

HRMS (ESI): $\mathrm{m} / \mathrm{z}[\mathrm{M}+\mathrm{H}]^{+}$calcd for $\mathrm{C}_{12} \mathrm{H}_{16} \mathrm{~N}_{3} \mathrm{O}_{2}: 234.1243$, found: 234.1238 


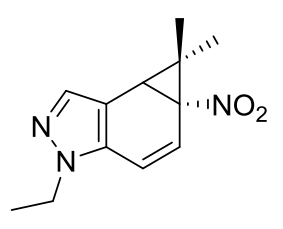

$3 a g$

Yield: $390 \mathrm{mg}$ (1.67 mmol at $2.02 \mathrm{mmol}$ scale, 83\%)

orange solid, $\mathrm{mp} 105.5^{\circ} \mathrm{C}$ (dec.)

eluent: toluene/ethyl acetate $10: 1$ to $3: 1$

$\mathrm{R}_{\mathrm{f}}=0.20$ (cyclohexane/ethyl acetate $3: 1$ )

${ }^{1} \mathrm{H}$ NMR (400 MHz, $\left.\mathrm{CDCl}_{3}\right): \delta=7.37(\mathrm{~d}, J=0.9 \mathrm{~Hz}, 1 \mathrm{H}), 6.65(\mathrm{dd}, J=10.3$, $1.0 \mathrm{~Hz}, 1 \mathrm{H}), 6.51(\mathrm{dd}, J=10.3,0.9 \mathrm{~Hz}, 1 \mathrm{H}), 4.09(\mathrm{qd}, J=7.3,0.8 \mathrm{~Hz}, 2 \mathrm{H})$, $3.52(\mathrm{~s}, 1 \mathrm{H}), 1.37-1.22(\mathrm{~m}, 6 \mathrm{H}), 0.58(\mathrm{~s}, 3 \mathrm{H})$.

${ }^{13} \mathrm{C}$ NMR $\left(100 \mathrm{MHz}, \mathrm{CDCl}_{3}\right): \delta=136.2,134.5,120.6,114.7,112.0,75.9$, $43.9,33.2,19.7,19.5,15.5,15.0$.

HRMS (ESI): $\mathrm{m} / \mathrm{z}[\mathrm{M}+\mathrm{H}]^{+}$calcd for $\mathrm{C}_{12} \mathrm{H}_{16} \mathrm{~N}_{3} \mathrm{O}_{2}: 234.1243$, found: 234.1237 


\subsection{Characterization of alkylated products}<smiles>Cc1ccc2ccccc2c1[N+](=O)[O-]</smiles>

2-methyl-1-nitronaphthalene, $4 a$

Yield: $263 \mathrm{mg}$ (1.41 mmol at $2.02 \mathrm{mmol}$ scale from 2a, 70\%)

$229 \mathrm{mg}(1.22 \mathrm{mmol}$ at $2.03 \mathrm{mmol}$ scale from $7 \mathrm{a}, 60 \%)$

$121 \mathrm{mg}(0.65 \mathrm{mmol}$ at $2.01 \mathrm{mmol}$ scale from $7 \mathrm{~b}, 32 \%)$

$171 \mathrm{mg}$ (0.91 mmol at $2.03 \mathrm{mmol}$ scale from 7c, 45\%)

orange crystals, mp $79.0-80.5^{\circ} \mathrm{C}$

eluent: cyclohexane/toluene $20: 1$ to $3: 1$

$\mathrm{R}_{\mathrm{f}}=0.39$ (cyclohexane/toluene $3: 1$ )

${ }^{1} \mathrm{H}$ NMR $\left(400 \mathrm{MHz}, \mathrm{CDCl}_{3}\right): \delta=7.86-7.80(\mathrm{~m}, 2 \mathrm{H}), 7.70(\mathrm{~d}, J=8.4 \mathrm{~Hz}, 1 \mathrm{H})$, $7.59-7.54(\mathrm{~m}, 1 \mathrm{H}), 7.55-7.46(\mathrm{~m}, 1 \mathrm{H}), 7.30(\mathrm{~d}, J=8.5 \mathrm{~Hz}, 1 \mathrm{H}), 2.47(\mathrm{~s}$, $3 \mathrm{H})$.

${ }^{13} \mathrm{C}$ NMR (100 MHz, $\left.\mathrm{CDCl}_{3}\right): \delta=147.7,132.1,130.4,128.4,128.0,127.9$, $127.4,126.6,124.6,121.1,17.8$.

${ }^{1} \mathrm{H} \mathrm{NMR}^{[16]},{ }^{13} \mathrm{CNMR}^{[17]}$ spectra and $\mathrm{mp}^{[18]}$ were consistent with those reported in the literature.<smiles>CCc1ccc2ccccc2c1[N+](=O)[O-]</smiles>

2-ethyl-1-nitronaphthalene, $\mathbf{4 b}$

Yield: $18 \mathrm{mg}(0.09 \mathrm{mmol}$ at $1.02 \mathrm{mmol}$ scale from $\mathbf{2 b}, \mathbf{9 \%})$

$125 \mathrm{mg}(0.62 \mathrm{mmol}$ at $2.02 \mathrm{mmol}$ scale from $7 \mathrm{~d}, 31 \%)$

$53 \mathrm{mg}(0.26 \mathrm{mmol}$ at $2.01 \mathrm{mmol}$ scale from $7 f, 13 \%)$

brown solid, $\mathrm{mp} 49.5-51.0^{\circ} \mathrm{C}$

eluent: cyclohexane/toluene $20: 1$ to $3: 1$

$R_{f}=0.42$ (cyclohexane/toluene $3: 1$ )

${ }^{1} \mathrm{H}$ NMR (400 MHz, $\left.\mathrm{CDCl}_{3}\right): \delta=7.87(\mathrm{~d}, J=8.5 \mathrm{~Hz}, 1 \mathrm{H}), 7.84(\mathrm{~d}, J=8.1 \mathrm{~Hz}$, $1 \mathrm{H}), 7.68(\mathrm{~d}, J=8.5 \mathrm{~Hz}, 1 \mathrm{H}), 7.62-7.52(\mathrm{~m}, 1 \mathrm{H}), 7.55-7.46(\mathrm{~m}, 1 \mathrm{H}), 7.37$ (d, $J=8.5 \mathrm{~Hz}, 1 \mathrm{H}), 2.76(\mathrm{q}, J=7.6 \mathrm{~Hz}, 2 \mathrm{H}), 1.31(\mathrm{t}, J=7.6 \mathrm{~Hz}, 3 \mathrm{H})$.

${ }^{13} \mathrm{C}$ NMR (100 MHz, $\left.\mathrm{CDCl}_{3}\right): \delta=147.3,133.0,132.2,130.5,128.4,127.9$, $126.63,126.61,124.5,121.2,25.0,15.1$.

${ }^{1} \mathrm{HNMR}^{[19]}$ and $\mathrm{mp}^{[20]}$ were consistent with those reported in the literature. 


\section{Further studies}

\subsection{Experiment with radical probe (TEMPO)}

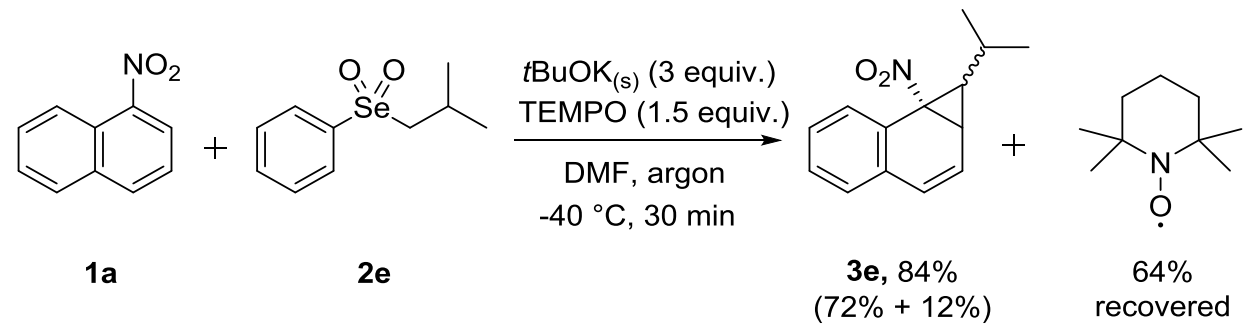

Experiment was carried out similarly to General procedure A with addition of 1.5 equiv. of TEMPO (2,2,6,6-tetramethylpiperidine-1-oxyl).

A $30 \mathrm{~mL}$ Schlenk tube was charged with nitronaphthalene (1a) (351 mg, $2.03 \mathrm{mmol}, 1.0$ equiv.), isobutyl phenyl selenone (2e) $(594 \mathrm{mg}, 2.42 \mathrm{mmol}, 1.2$ equiv.), TEMPO (476 mg, $3.05 \mathrm{mmol}, 1.5$ equiv.) and flushed with argon. Dry DMF ( $8 \mathrm{~mL}$ ) was added and after dissolution of substrates mixture was cooled to $-40^{\circ} \mathrm{C}$. Solid potassium tert-butoxide (688 mg, $6.13 \mathrm{mmol}, 3.0$ equiv.) was added in one portion. After 30 min reaction mixture was quenched with $\mathrm{NH}_{4} \mathrm{Cl}_{\mathrm{aq}}(10 \mathrm{~mL}, 10 \% \mathrm{w} / \mathrm{v})$. Mixture was transferred to separatory funnel, $\mathrm{NH}_{4} \mathrm{Cl}_{\mathrm{aq}}(50 \mathrm{~mL}, 10 \% \mathrm{w} / \mathrm{v})$ was added, mixture was extracted with ethyl acetate $(3 \times 50 \mathrm{~mL})$, combined organic phases were washed with $\mathrm{LiCl}_{\mathrm{aq}}(50 \mathrm{~mL}$, $1.0 \mathrm{M}), \mathrm{H}_{2} \mathrm{O}(50 \mathrm{~mL})$, brine $(50 \mathrm{~mL})$, and dried over anhydrous $\mathrm{MgSO}_{4}$. Mixture was filtered, evaporated and separated with column chromatography (length $44 \mathrm{~cm}$, diameter $3 \mathrm{~cm}, 260 \mathrm{~mL}$ of silica gel) with eluent cyclohexane/toluene $20: 1$ to $3: 1$, then toluene to toluene/ethyl acetate 20:1 yielding endo-3e (334 mg, $1.46 \mathrm{mmol}, 72 \%)$, exo-3e (55 mg, $0.24 \mathrm{mmol}, 12 \%)$, and recovered TEMPO (305 mg, $1.95 \mathrm{mmol}, 64 \%$ recovery). 


\subsection{NMR signals assignment for endo- and exo-benzonorcaradienes}

${ }^{1} \mathrm{H}$ NMR and ${ }^{13} \mathrm{C}$ NMR signals were assigned for endo-3e and exo-3e by COSY, HSQC and HMBC experiments. Also X-Ray studies were done for both isomers, what ensured us about correlation between NMR spectra and configuration of the product. On this basis, since NMR spectra of all benzonorcaradienes display similar signals patterns we were able to assign endo/exo structure to all obtained compounds.

In case of carbon signals situated very close on the spectrum unambiguous assignment was not possible, therefore some signals are given in brackets.
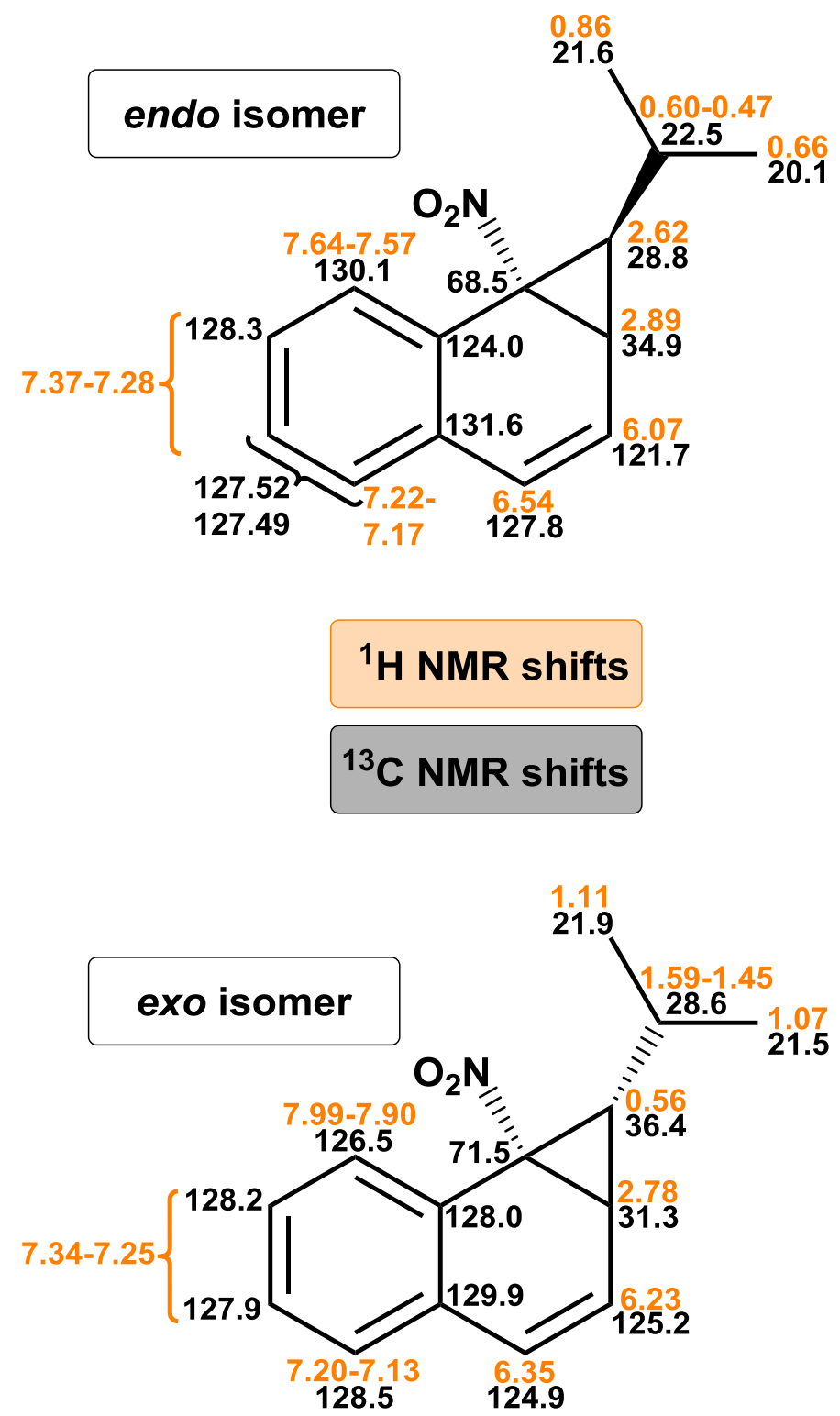

Endo isomers of benzonorcaradienes have two protons adjacent to vicinal carbon atoms of cyclopropane ring in cis configuration. Coupling constants measured for endo-3e and endo-3q are $11.5 \mathrm{~Hz}$ and $11.2 \mathrm{~Hz}$, respectively. For exo-3e and exo-3q, which have two protons in trans configuration coupling constants are $7.2 \mathrm{~Hz}$ and $7.6 \mathrm{~Hz}$, respectively. In case of 3a, which has three protons adjacent to cyclopropane ring coupling constants for cis, trans and geminal protons are 11.1 $\mathrm{Hz}, 7.1 \mathrm{~Hz}$ and $5.2 \mathrm{~Hz}$, respectively. 

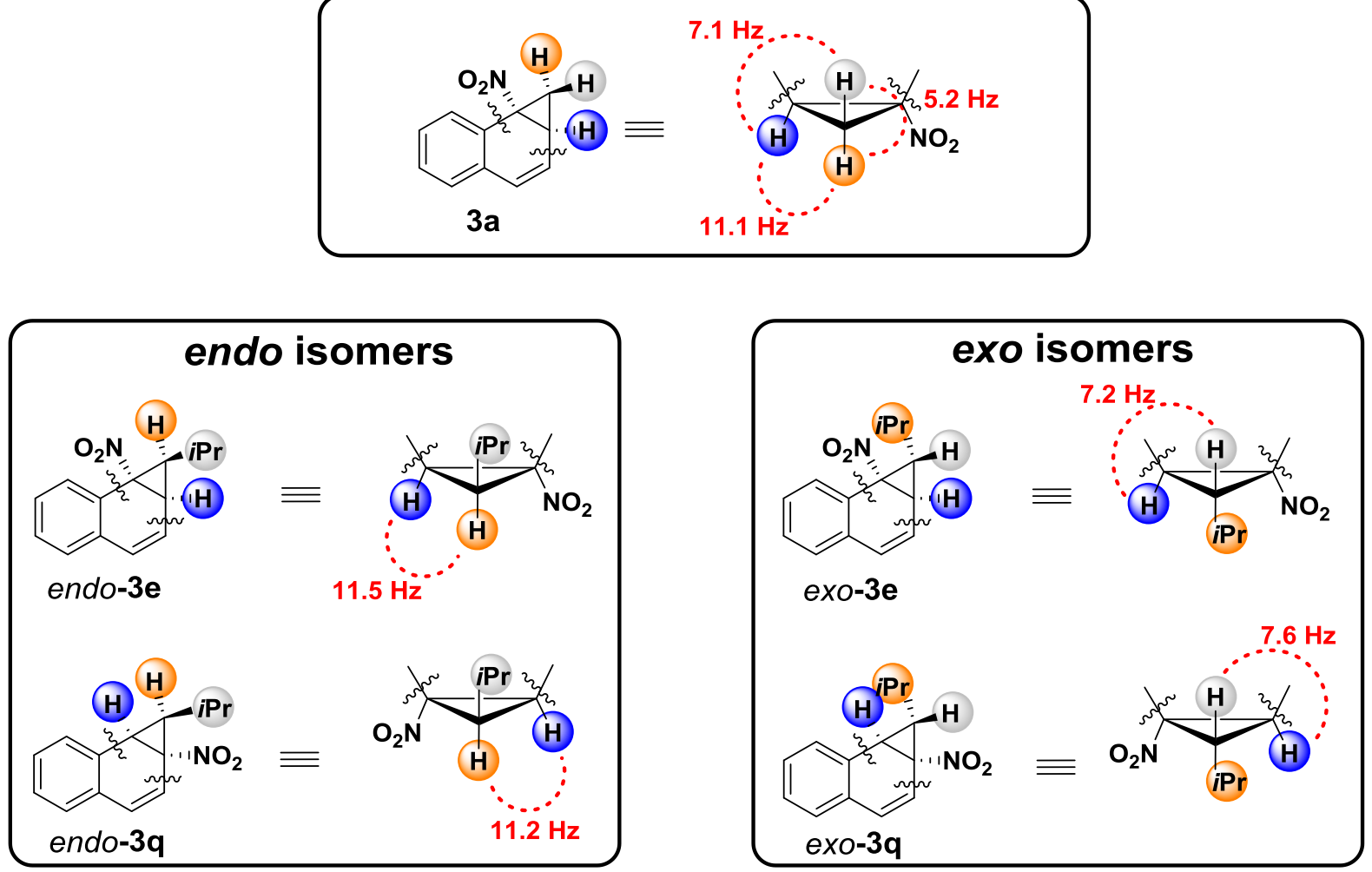

\begin{abstract}
${ }^{1} \mathrm{H}$ NMR spectra of benzonorcaradienes display a few characteristic features, which may be helpful in distinguishing between endo and exo isomer for a given compound. The most distinctive is chemical shift of protons belonging to the cyclopropane ring (shown in orange and gray), for which chemical shift can be easily established by COSY. Groups trans to $\mathrm{NO}_{2}$ group are shifted to the upfield (from 0.95 to $0.49 \mathrm{ppm}$ ), while groups cis to $\mathrm{NO}_{2}$ group are shifted to the downfield (from 2.98 to $1.87 \mathrm{ppm}$ for hydrogen atoms and from 1.87 to $1.38 \mathrm{ppm}$ for alkyl groups). Another characteristic feature is difference in chemical shifts between two vinyl protons. For benzonorcaradienes obtained from 1-nitronaphthalene (and 5-bromo-1-nitronaphthalene) difference between chemical shifts of vinyl protons is bigger for endo isomers than for exo isomers, whereas for 2-nitronaphthalene the trend is reversed, i.e. difference between chemical shifts of vinyl protons is bigger for exo isomers than for endo isomers.
\end{abstract}




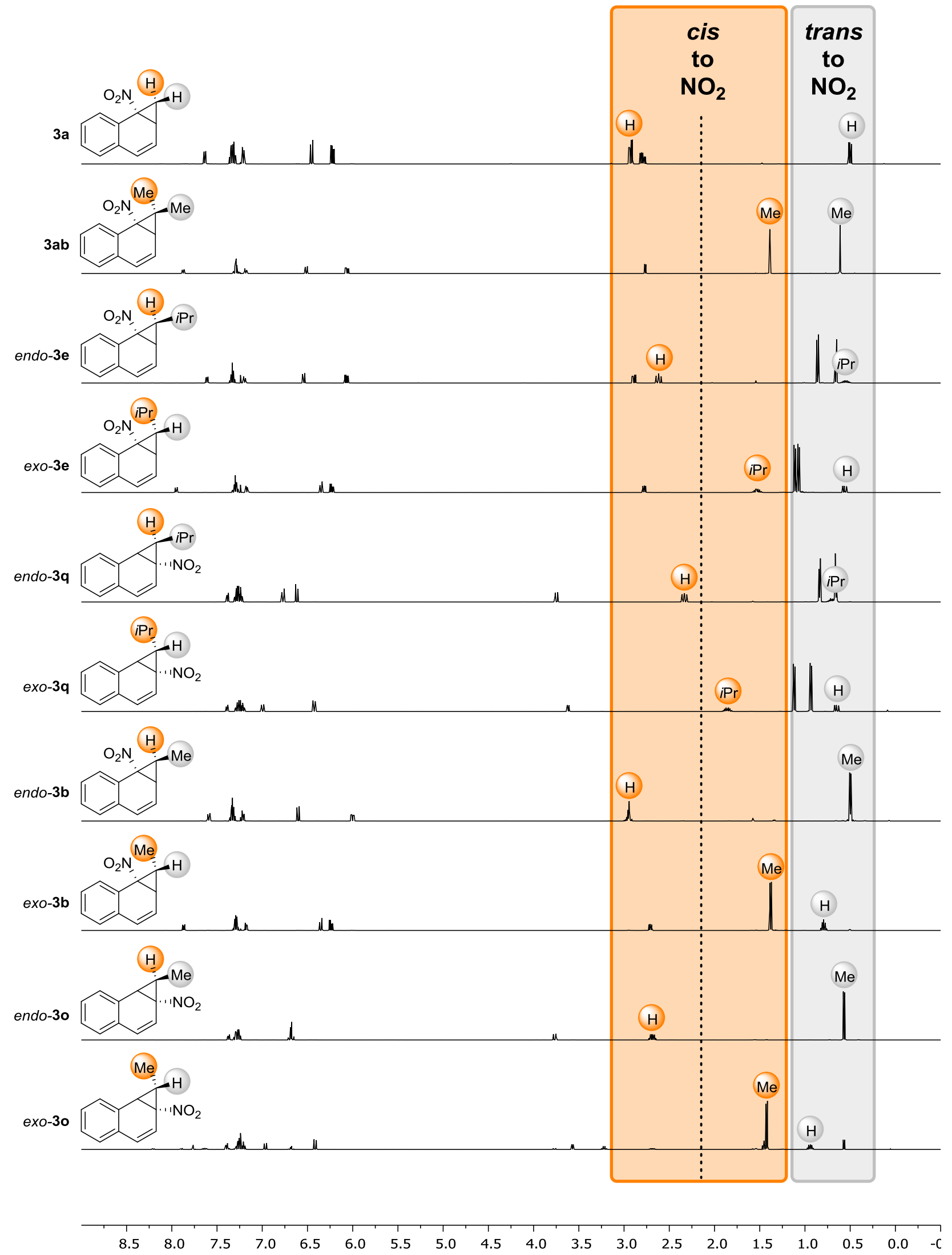




\subsection{Synthesis of protonated $\sigma^{\mathrm{H}}$ adduct (6)}

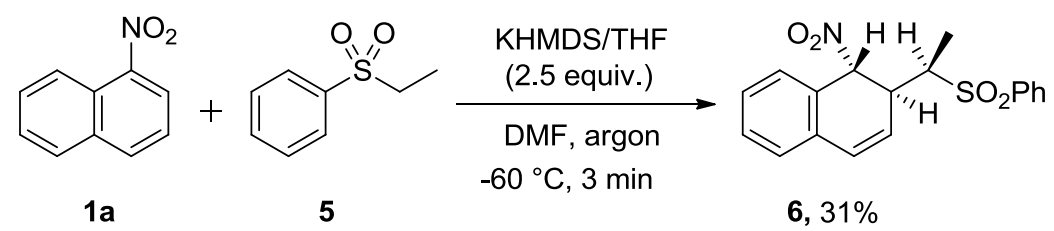

A $30 \mathrm{~mL}$ Schlenk tube was charged with nitronaphthalene (1a) (348 mg, $2.01 \mathrm{mmol}, 1.0$ equiv.), ethyl phenyl sulfone (5) (412 mg, $2.42 \mathrm{mmol}, 1.2$ equiv.), and flushed with argon. Dry DMF (6 mL) was added and after dissolution of substrates mixture was cooled to $-60{ }^{\circ} \mathrm{C}$. KHMDS solution in THF (5.6 $\mathrm{mL}, 5.0 \mathrm{mmol}, 20 \% \mathrm{w} / \mathrm{w}, 2.5$ equiv.) was added. After 3 min reaction mixture was slowly poured onto vigorously stirred $\mathrm{NH}_{4} \mathrm{Cl}_{\text {aq }}(40 \mathrm{~mL}, 10 \% \mathrm{w} / \mathrm{v})$ and immediately after that Schlenk tube was rinsed with $\mathrm{NH}_{4} \mathrm{Cl}_{\mathrm{aq}}(10 \mathrm{~mL}, 10 \% \mathrm{w} / \mathrm{v})$. Mixture was transferred to separatory funnel, extracted with ethyl acetate $(3 \times 50 \mathrm{~mL})$, combined organic phases were washed with $\mathrm{LiCl}_{\mathrm{aq}}(50 \mathrm{~mL}, 1.0 \mathrm{M}), \mathrm{H}_{2} \mathrm{O}(50 \mathrm{~mL})$, brine $(50$ $\mathrm{mL}$ ), and dried over anhydrous $\mathrm{MgSO}_{4}$. Mixture was filtered, evaporated and separated with column chromatography (length $28 \mathrm{~cm}$, diameter $3 \mathrm{~cm}, 200 \mathrm{~mL}$ of silica gel) with eluent toluene to toluene/ethyl acetate 50:1 giving slightly impure crude product, which was recrystallized by slow evaporation of its solution in dichlormethane/heptane mixture yielding protonated adduct 6 (216 $\mathrm{mg}, 0.63 \mathrm{mmol}, \mathbf{3 1 \%}$ ) as dark orange crystals.

${ }^{1} \mathrm{H}$ NMR of crude reaction mixture after quench revealed that it consisted mainly of protonated $\sigma$-adduct (6), unreacted ethyl phenyl sulfone (5) and one minor product (some of its signals were assigned by COSY spectrum). The minor product could be second diastereoisomer of the protonated $\sigma$-adduct, however unequivocal proof is missing. The product decomposed or isomerized on silica gel chromatography, thus we did not characterized it in detail. 

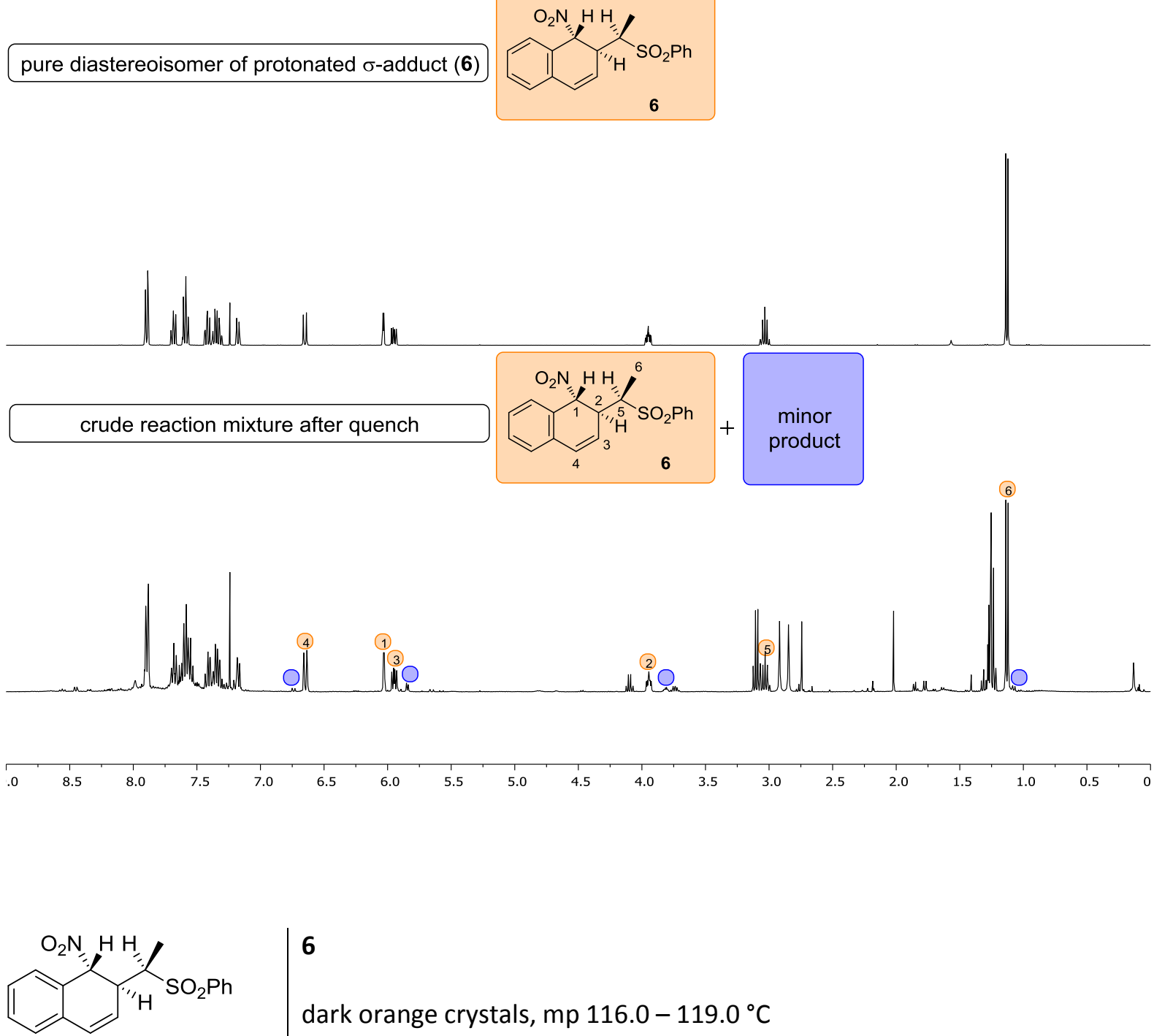

dark orange crystals, $\mathrm{mp} 116.0-119.0^{\circ} \mathrm{C}$

eluent: toluene then toluene/ethyl acetate $50: 1$

$\mathrm{R}_{\mathrm{f}}=0.22$ (cyclohexane/ethyl acetate $3: 1$ )

${ }^{1} \mathrm{H}$ NMR (400 MHz, $\left.\mathrm{CDCl}_{3}\right): \delta=7.91-7.87(\mathrm{~m}, 2 \mathrm{H}), 7.73-7.64(\mathrm{~m}, 1 \mathrm{H})$, $7.62-7.55(\mathrm{~m}, 2 \mathrm{H}), 7.42(\mathrm{td}, J=7.3,1.7 \mathrm{~Hz}, 1 \mathrm{H}), 7.38-7.30(\mathrm{~m}, 2 \mathrm{H}), 7.18$ (dd, $J=7.4,1.2 \mathrm{~Hz}, 1 \mathrm{H}), 6.65(\mathrm{~d}, J=9.8 \mathrm{~Hz}, 1 \mathrm{H}), 6.03(\mathrm{~d}, J=2.8 \mathrm{~Hz}, 1 \mathrm{H})$, $5.95(\mathrm{dd}, J=9.8,5.7 \mathrm{~Hz}, 1 \mathrm{H}), 3.95$ (ddd, $J=7.3,5.6,2.8 \mathrm{~Hz}, 1 \mathrm{H}), 3.03(\mathrm{p}, J=$ $7.1 \mathrm{~Hz}, 1 \mathrm{H}), 1.13(\mathrm{~d}, J=7.1 \mathrm{~Hz}, 3 \mathrm{H})$.

${ }^{13} \mathrm{C}$ NMR (100 MHz, $\mathrm{CDCl}_{3}$ ): $\delta=136.4,134.2,132.8,131.1,131.0,129.4$, $129.3,129.1,128.6,127.1,125.5,124.1,86.3,60.2,37.1,12.4$.

HRMS (ESI): $\mathrm{m} / \mathrm{z}[\mathrm{M}+\mathrm{Na}]^{+}$calcd for $\mathrm{C}_{18} \mathrm{H}_{17} \mathrm{NO}_{4} \mathrm{SNa}$ : 366.0776; found: 366.0775 


\subsection{Endo-exo isomerisation of benzonorcaradienes}

$5 \mathrm{mg}$ of each of benzonorcaradienes were dissolved in toluene- $d_{8}(0.8 \mathrm{~mL})$ and resulted solutions were transferred to NMR tubes. NMR tubes were immersed in oil bath $\left(90^{\circ} \mathrm{C}\right)$. For indicated times NMR tubes were taken out of oil bath, cooled on air and measured by NMR.

We have not isolated exo-3g and exo-3o in pure state, because they were inseparable from other impurities and their amount was small. However, we used them for isomerisation studies, as they contained only exo isomer and alkylated product that should not affect the isomerisation reaction. ${ }^{1} \mathrm{HNMR}$ spectra displayed the same characteristic pattern of signals as for all other exo-benzonorcaradienes, what ensured us about identity of exo-3g and exo-3o.
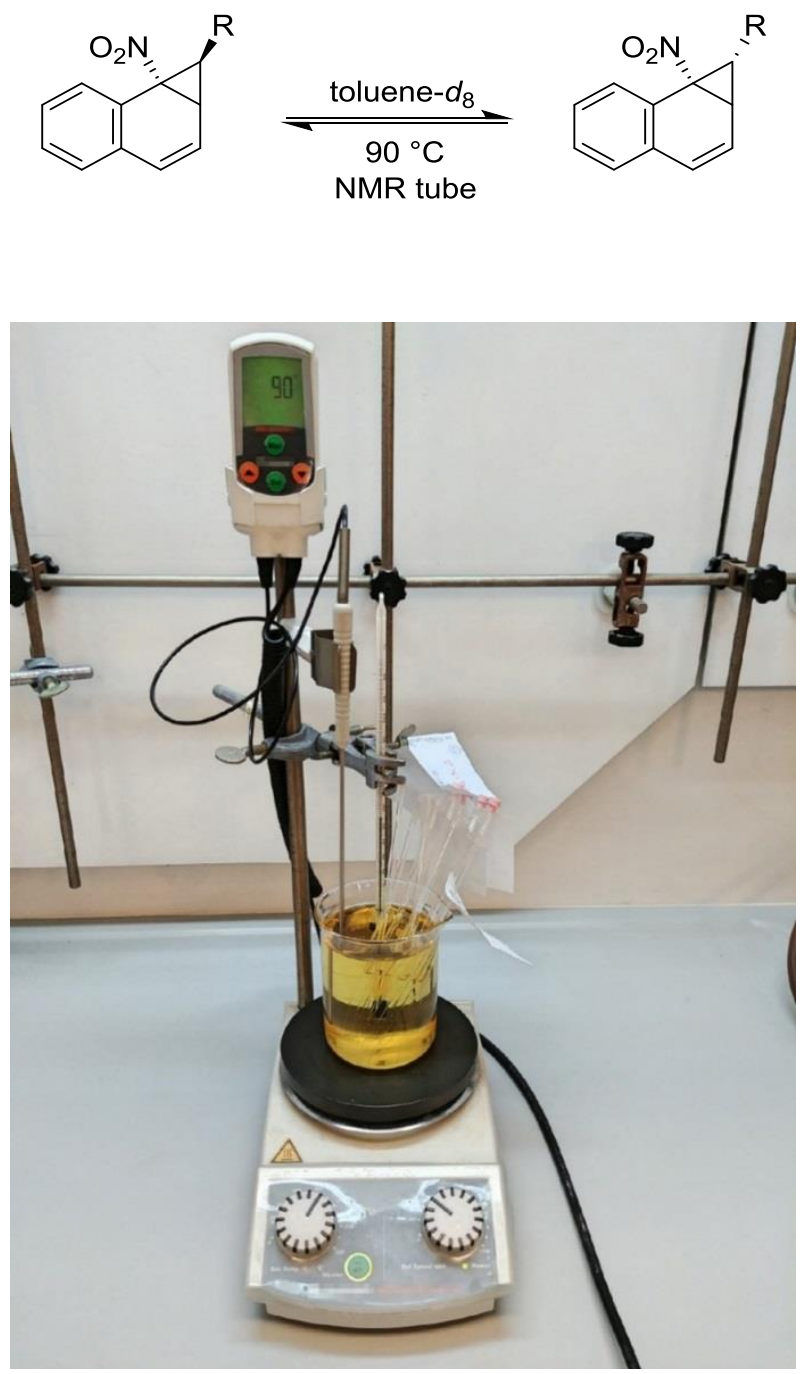

On the chart below results for exo isomers of $\mathbf{3 b}, \mathbf{3 e}, \mathbf{3 g}, \mathbf{3 0}, \mathbf{3 q}$, and $\mathbf{3 s}$ are presented. The major factor that affects isomerisation rate is size of the substituent adjacent to cyclopropane ring. Methyl substituted benzonorcaradienes ( $\mathbf{3 b}$ and $\mathbf{3 0}$ ) isomerize fastest, isopropyl substituted (3e and $\mathbf{3 q}$ ) isomerize at moderate rate, while $t$-butyl substituted hardly isomerize under these conditions. The minor factor is position of $\mathrm{NO}_{2}$ group - benzonorcaradienes obtained from 2-nitronaphthalene isomerize slightly faster than these obtained from 1-nitronaphthalene. 
For $\mathbf{3 0}$ isomerisation was particularly fast, that explained problem with isolation of exo-3o in pure form.

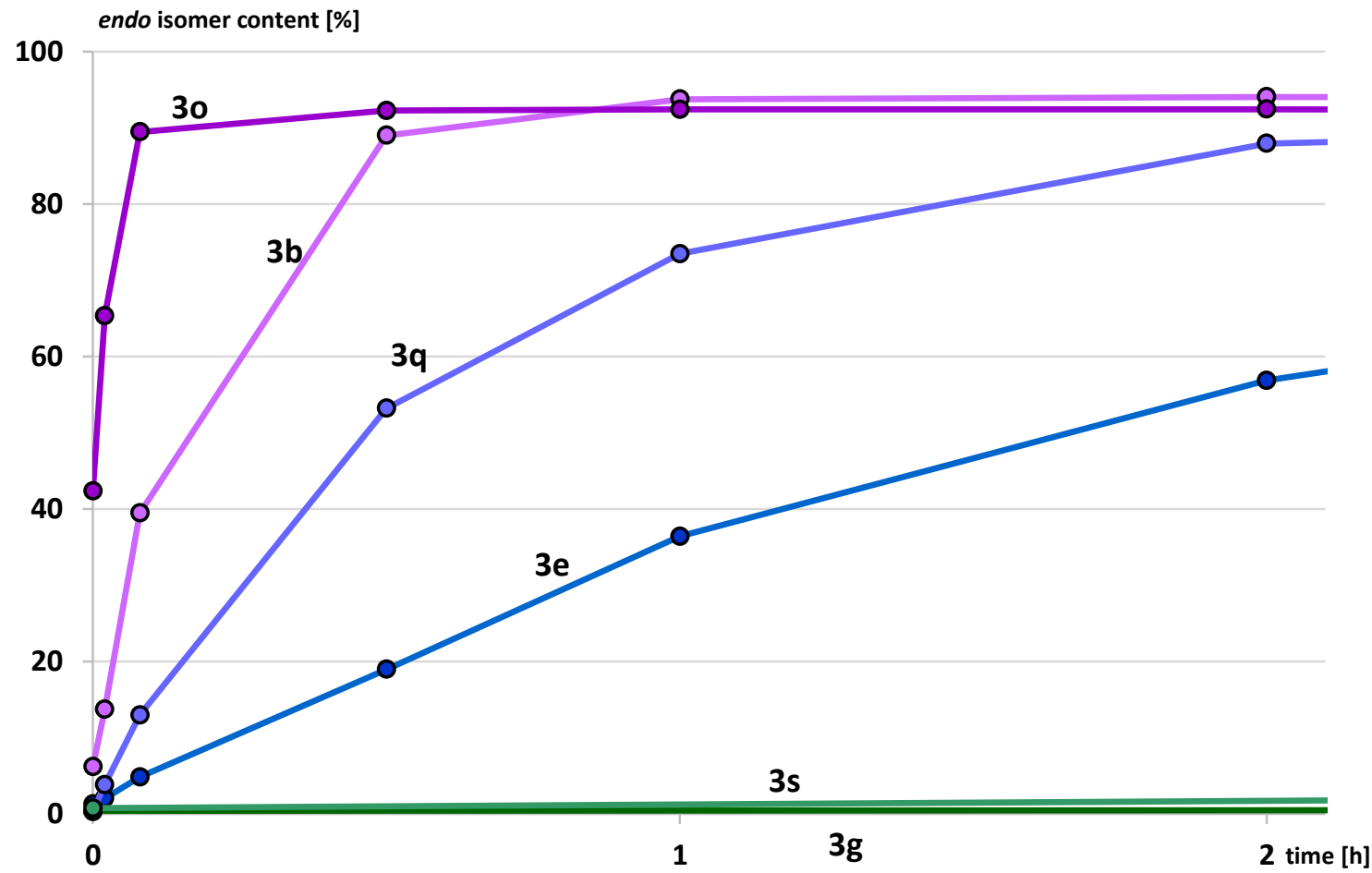

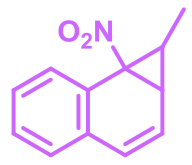

$3 b$<smiles>CC1C2c3ccccc3C=CC12[N+](=O)[O-]</smiles>

30<smiles>CC(C)C1C2C=Cc3ccccc3C21[N+](=O)[O-]</smiles>

$3 e$<smiles>CC(C)C1C2c3ccccc3C=CC21[N+](=O)[O-]</smiles>

$3 q$<smiles>CC(C)(C)C1C2C=Cc3ccccc3C21NO</smiles>

$3 \mathrm{~g}$<smiles>CC(C)(C)C1C2c3ccccc3C=CC21[N+](=O)[O-]</smiles>

3s

For all isomers with methyl and isopropyl substituent adjacent to cyclopropane ring we observed that equilibrium is strongly shifted towards endo isomers (from 91:9 for 3q to 94:6 for 3e). Isomerisations of benzonorcaradienes with tert-butyl substituent adjacent to cyclopropane ring, $\mathbf{3 g}$ and $3 s$, were too slow to reach equilibrium at this temperature $\left(90^{\circ} \mathrm{C}\right)$. 


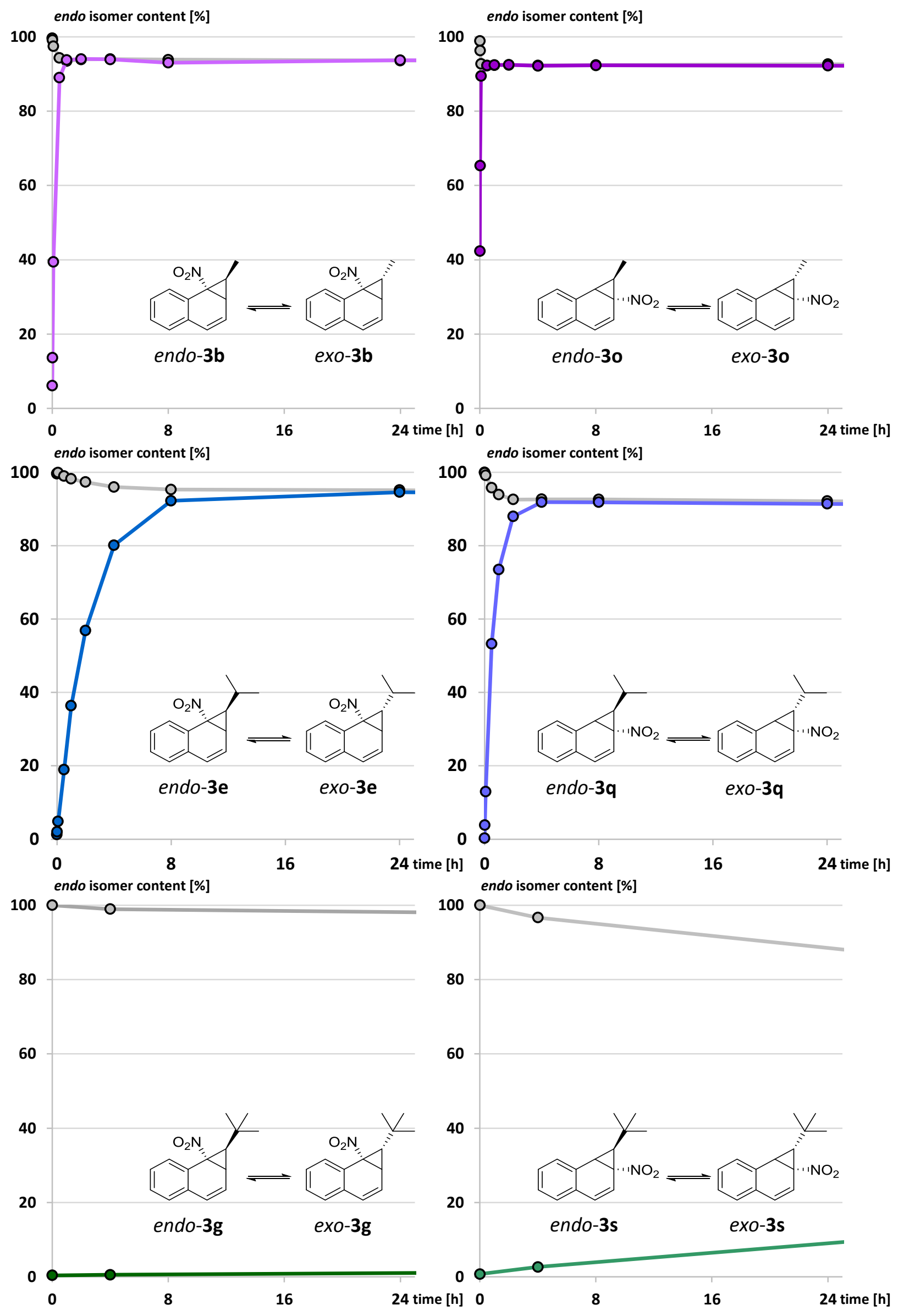


Isomerizations of $3 \mathrm{~g}$ and $3 \mathrm{~s}$ were repeated at $150^{\circ} \mathrm{C}$.

$5 \mathrm{mg}$ of each of benzonorcaradienes were dissolved in toluene- $d_{8}(0.8 \mathrm{~mL})$ and resulted solutions were transferred to NMR tubes equipped with screw caps. Tubes were immersed in oil bath $\left(150{ }^{\circ} \mathrm{C}\right)$. For indicated times tubes were taken out of oil bath, cooled on air and measured by NMR.

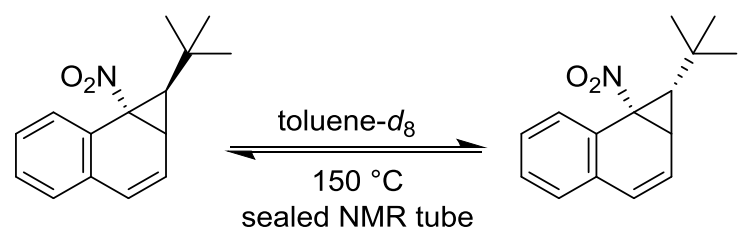

For $\mathbf{3 g}$ we were not able to reach equilibrium, however it became clear that in both cases the equilibrium is slightly shifted towards exo isomers.

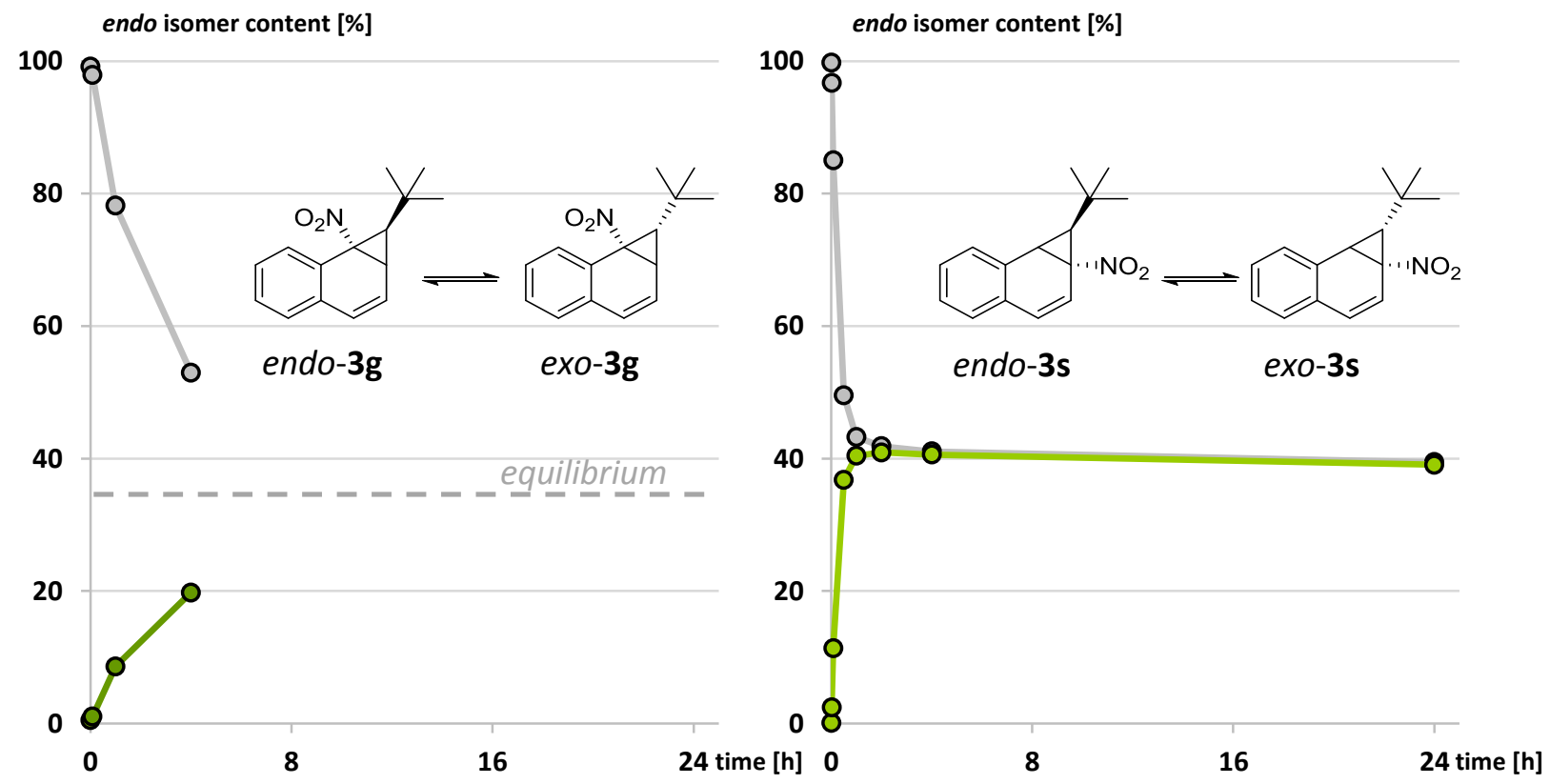


Tables below contain data for all isomerisations given as percentage content of endo isomer in reaction mixtures. For every pair of isomers starting isomer was given at the top of the table.

$90{ }^{\circ} \mathrm{C}$ for Me and $\operatorname{Pr}$ substituted benzonorcaradienes:
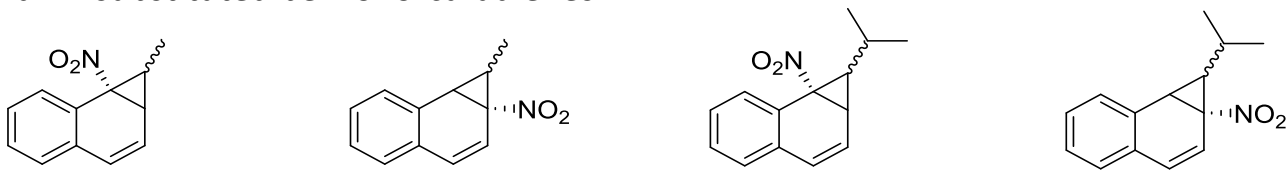

\begin{tabular}{|c|c|c|c|c|c|c|c|c|}
\hline time & endo-3b & exo-3b & endo-3o & exo-3o & endo-3e & exo-3e & endo-3q & $e x o-3 q$ \\
\hline 0 & 100 & 6 & 99 & 42 & 100 & 1 & 100 & 0 \\
\hline $1 \mathrm{~min}$ & 99 & 14 & 96 & 65 & 100 & 2 & 100 & 4 \\
\hline $5 \mathrm{~min}$ & 97 & 39 & 93 & 89 & 100 & 5 & 99 & 13 \\
\hline $0.5 \mathrm{~h}$ & 94 & 89 & 92 & 92 & 99 & 19 & 96 & 53 \\
\hline $1 \mathrm{~h}$ & 93 & 94 & 92 & 92 & 98 & 36 & 94 & 73 \\
\hline $2 \mathrm{~h}$ & 94 & 94 & 92 & 92 & 97 & 57 & 93 & 88 \\
\hline $4 \mathrm{~h}$ & 94 & 94 & 92 & 92 & 96 & 80 & 93 & 92 \\
\hline $8 \mathrm{~h}$ & 94 & 93 & 92 & 92 & 95 & 92 & 93 & 92 \\
\hline $24 \mathrm{~h}$ & 94 & 94 & 93 & 92 & 95 & 95 & 92 & 91 \\
\hline $48 \mathrm{~h}$ & 93 & 93 & 92 & 92 & 94 & 94 & 91 & 91 \\
\hline
\end{tabular}

$90{ }^{\circ} \mathrm{C}$ for $t$ Bu substituted benzonorcaradienes:

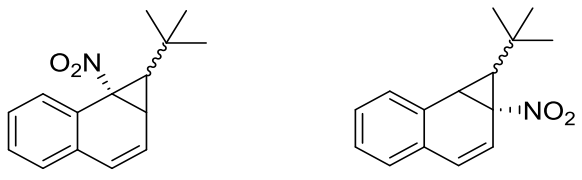

\begin{tabular}{|r|rr|rr|}
\hline time & endo-3g & exo-3g & endo-3s & exo-3s \\
\hline $\mathbf{0}$ & 100 & 0 & 100 & 1 \\
\hline $\mathbf{4 h}$ & 99 & 1 & 97 & 3 \\
\hline $\mathbf{9 3} \mathbf{~ h}$ & 95 & 3 & 60 & 31 \\
\hline
\end{tabular}

$150{ }^{\circ} \mathrm{C}$ for $t$ Bu substituted benzonorcaradienes:

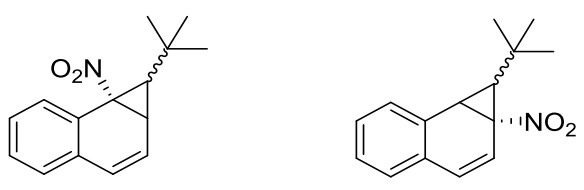

\begin{tabular}{|r|rr|rr|}
\hline \multicolumn{1}{|c|}{ time } & endo-3g & exo-3g & endo-3s & exo-3s \\
\hline $\mathbf{0}$ & 99 & 1 & 100 & 0 \\
\hline $\mathbf{1} \mathbf{~} \mathbf{m i n}$ & - & - & 97 & $\mathbf{2}$ \\
\hline $\mathbf{5} \mathbf{~}$ in & 98 & 1 & 85 & 11 \\
\hline $\mathbf{0 . 5} \mathbf{~ h}$ & - & - & 50 & 37 \\
\hline $\mathbf{1 ~ h}$ & 78 & 9 & 43 & 40 \\
\hline $\mathbf{2} \mathbf{~}$ & - & - & 42 & 41 \\
\hline $\mathbf{4} \mathbf{~ h}$ & 53 & 20 & 41 & 41 \\
\hline $\mathbf{2 4} \mathbf{~ h}$ & - & - & 39 & 39 \\
\hline
\end{tabular}




\subsection{Synthesis of cyclopropa[a]naphthalene (8)}

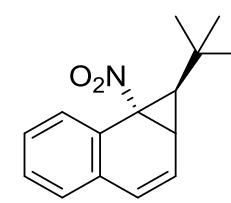

endo-3g

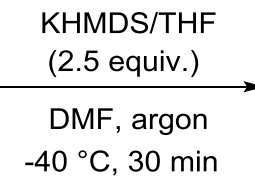

$-40^{\circ} \mathrm{C}, 30 \mathrm{~min}$

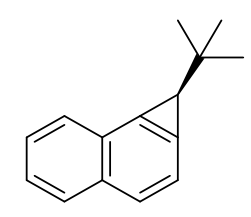

8, quant.

A $30 \mathrm{~mL}$ Schlenk tube was charged with endo-3g (122 mg, $0.50 \mathrm{mmol}, 1.0$ equiv.) and flushed with argon. Dry DMF $(1.5 \mathrm{~mL})$ was added and after dissolution of substrate mixture was cooled to $-40{ }^{\circ} \mathrm{C}$. KHMDS solution in THF ( $1.4 \mathrm{~mL}, 1.26 \mathrm{mmol}, 20 \% \mathrm{w} / \mathrm{w}, 2.5$ equiv.) was added. After 30 min reaction mixture was quenched with $\mathrm{NH}_{4} \mathrm{Cl}_{\mathrm{aq}}(2.5 \mathrm{~mL}, 10 \% \mathrm{w} / \mathrm{v})$. Mixture was transferred to separatory funnel, $\mathrm{NH}_{4} \mathrm{Cl}_{\text {aq }}(50 \mathrm{~mL}, 10 \% \mathrm{w} / \mathrm{v})$ was added, mixture was extracted with ethyl acetate $(3 \times 50 \mathrm{~mL})$, combined organic phases were washed with $\mathrm{LiCl}_{\mathrm{aq}}(50 \mathrm{~mL}, 1.0 \mathrm{M}), \mathrm{H}_{2} \mathrm{O}(50 \mathrm{~mL})$, brine $(50 \mathrm{~mL})$ and dried over anhydrous $\mathrm{MgSO}_{4}$. Mixture was filtered, evaporated and dried under high vacuum yielding cycloproparene 8 ( $98 \mathrm{mg}, 0.50 \mathrm{mmol}$, quant.) as a yellow oil.

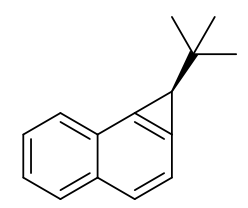

1-tert-butyl-1H-cyclopropa[a]naphthalene, 8

yellow oil

$R_{f}=0.60$ (cyclohexane/ethyl acetate $20: 1$ )

${ }^{1} \mathbf{H}$ NMR (400 MHz, $\left.\mathrm{CDCl}_{3}\right): \delta=7.98-7.90(\mathrm{~m}, 1 \mathrm{H}), 7.77-7.73(\mathrm{~m}, 2 \mathrm{H})$, $7.59-7.50(\mathrm{~m}, 1 \mathrm{H}), 7.52(\mathrm{~d}, J=7.0 \mathrm{~Hz}, 1 \mathrm{H}), 7.42(\mathrm{~m}, 1 \mathrm{H}), 3.92(\mathrm{~s}, 1 \mathrm{H}), 0.91$ $(\mathrm{s}, 9 \mathrm{H})$.

${ }^{13} \mathrm{C}$ NMR (100 MHz, $\left.\mathrm{CDCl}_{3}\right): \delta=135.1,130.5,130.2,129.8,129.4,127.5$, $125.1,124.7,124.5,115.5,49.7,34.0,28.8$.

MS (EI): m/z (\%) = $196\left(17,[M]^{+}\right), 181\left(60,\left[M-\mathrm{CH}_{3}\right]^{+}\right), 139\left(100,\left[\mathrm{M}-\mathrm{C}_{4} \mathrm{H}_{9}\right]^{+}\right)$

HRMS (EI): $\mathrm{m} / \mathrm{z}[\mathrm{M}]^{+}$calcd for $\mathrm{C}_{15} \mathrm{H}_{16}$ : 196.1252; found: 196.1255

Cycloproparene 8 was surprisingly stable during workup and as $\mathrm{CDCl}_{3}$ solution for several hours. We observed signs of decomposition on ${ }^{1} \mathrm{H}$ NMR spectrum after one night in NMR tube.

By comparing of ${ }^{1} \mathrm{H}$ NMR spectra of benzonorcaradiene endo-3g and cycloproparene 8 we observed that coupling between one vinyl proton and one proton adjacent to cyclopropane ring disappears after the reaction, due to loss of cyclopropyl proton in a $\mathrm{HNO}_{2}$ form. Two vinyl protons shifted for ca. $1.5 \mathrm{ppm}$ to the left, from vinyl protons region to aromatic protons region. 


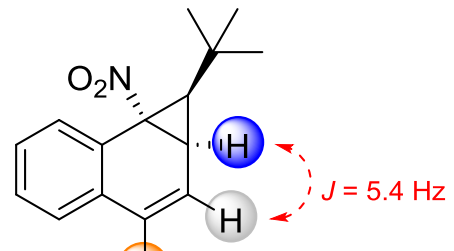
endo-3g
H)

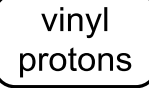<smiles>O=[N+]([O-])O</smiles>

8 protons

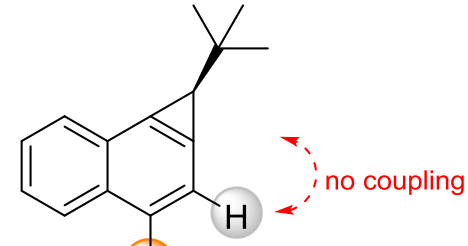

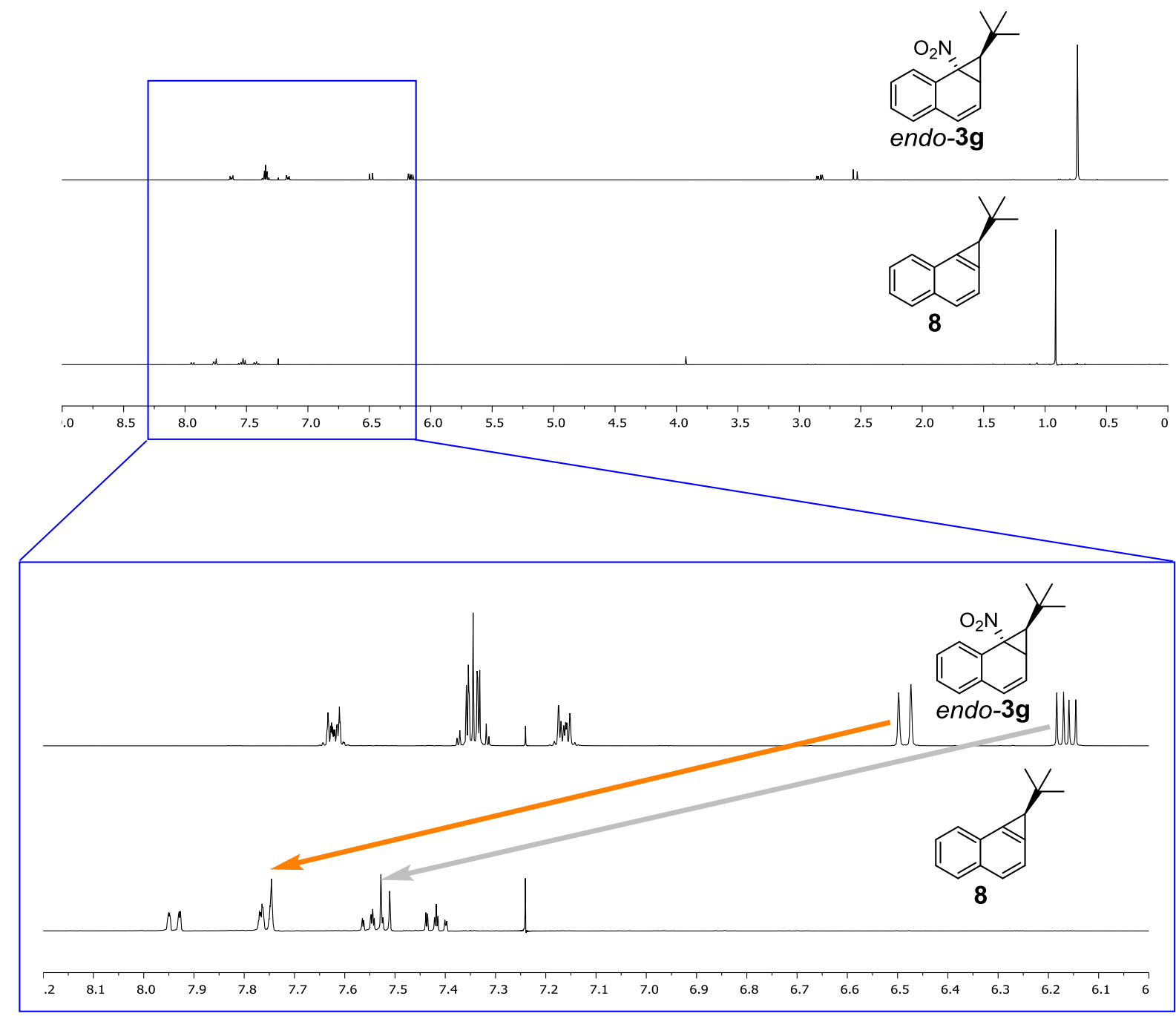

Attempt at purification of $\mathbf{8}$ by column chromatography led to a mixture of two much more polar products. ${ }^{1} \mathrm{H}$ and ${ }^{13} \mathrm{C}$ NMR spectra suggested that the mixture contains two isomeric benzyl alcohols, 2,2-dimethyl-1-(1-naphthyl)propanol and 2,2-dimethyl-1-(2-naphthyl)propanol. ${ }^{1} \mathrm{H}$ NMR spectrum of the mixture was consistent with those reported in the literature for 2,2-dimethyl-1-(1-naphthyl)propanol ${ }^{[21,22]}$ and 2,2-dimethyl-1-(2-naphthyl)propanol ${ }^{[21]}$. Most probably the products were formed by reaction with water adsorbed on silica gel. Identity of both products was additionally confirmed by GC-MS analysis of the mixture. 


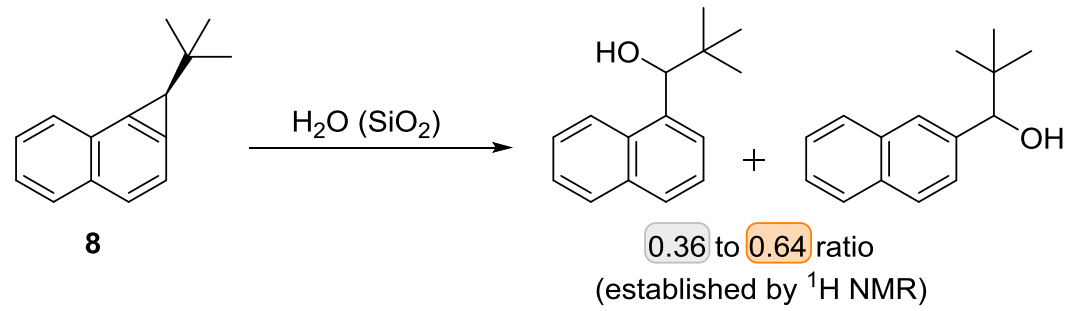

\section{${ }^{1} \mathrm{H}$ NMR spectrum:}
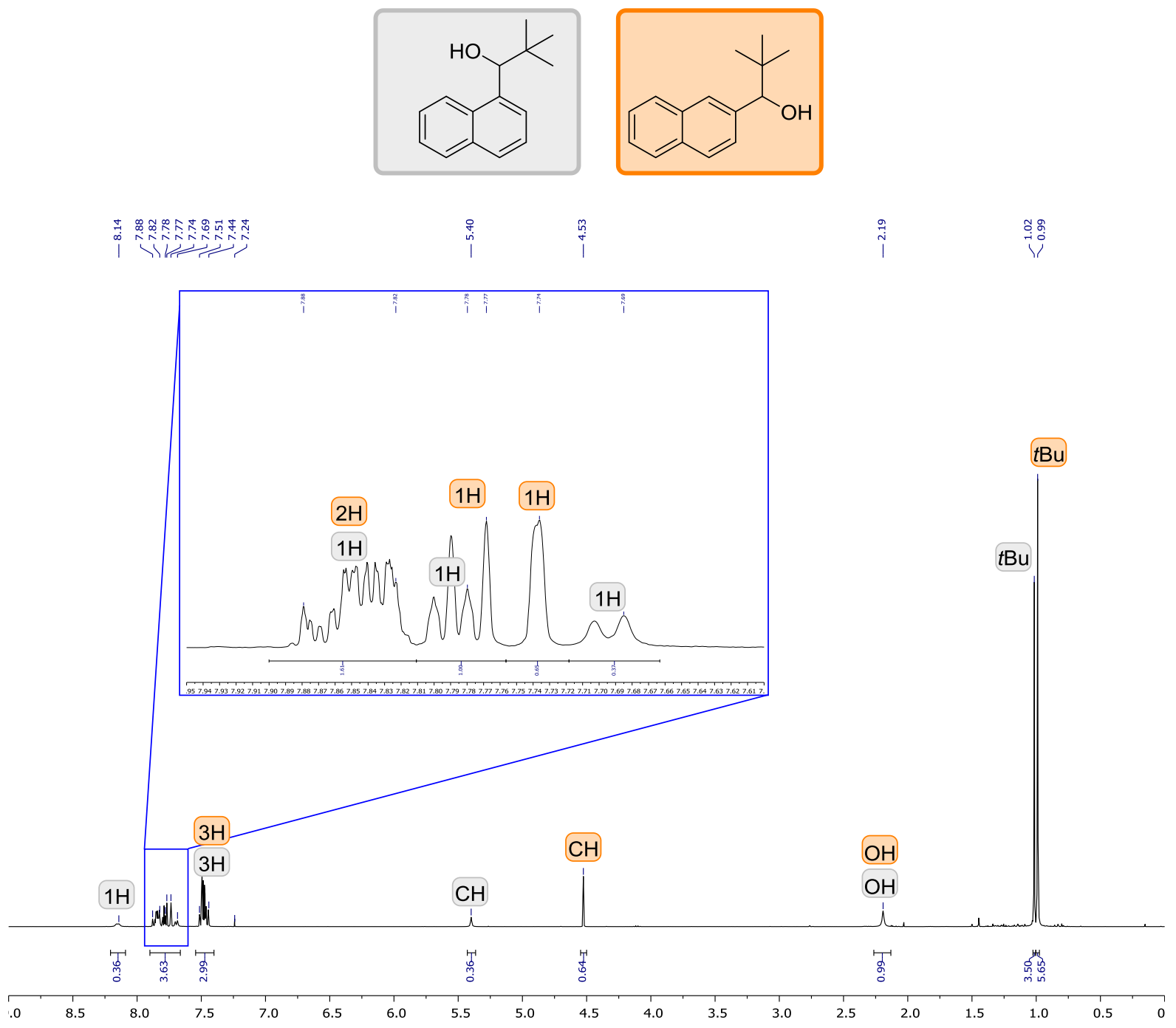
GC-MS:
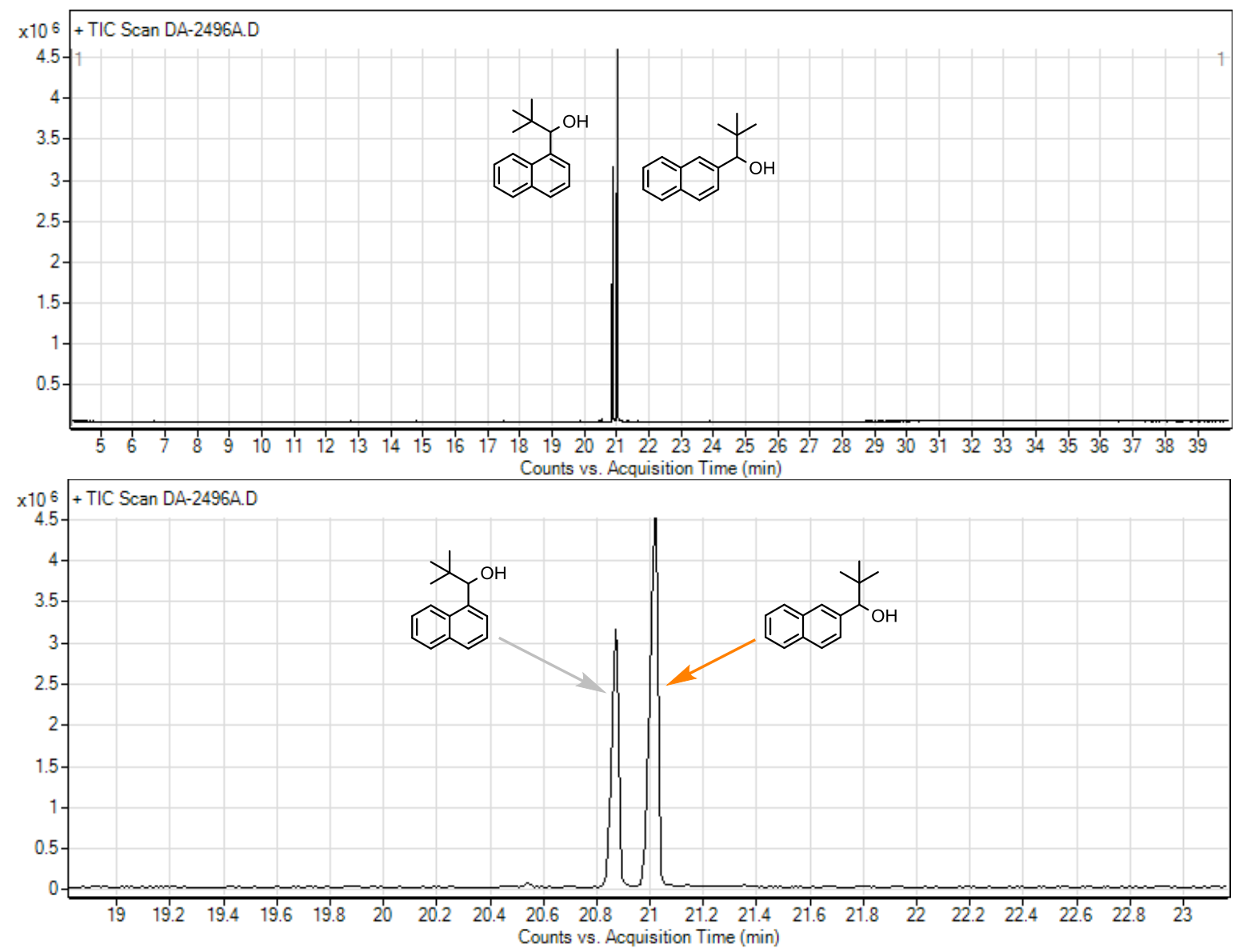

peak

retention
time [min]

structure

\begin{tabular}{|c|c|c|c|}
\hline 1 & 20.869 & 37.83 & $\begin{array}{l}214\left(6,[\mathrm{M}]^{+}\right) \\
196\left(6,\left[\mathrm{M}-\mathrm{H}_{2} \mathrm{O}\right]^{+}\right) \\
181\left(16,\left[\mathrm{M}-\mathrm{H}_{2} \mathrm{O}-\mathrm{CH}_{3}\right]^{+}\right) \\
157\left(100,\left[\mathrm{M}-\mathrm{C}_{4} \mathrm{H}_{9}\right]^{+}\right) \\
129\left(92,\left[\mathrm{M}-\mathrm{C}_{5} \mathrm{H}_{9} \mathrm{O}\right]^{+}\right)\end{array}$ \\
\hline 2 & 21.024 & 62.17 & $\begin{array}{l}214\left(7,[\mathrm{M}]^{+}\right) \\
196\left(5,\left[\mathrm{M}-\mathrm{H}_{2} \mathrm{O}\right]^{+}\right) \\
181\left(10,\left[\mathrm{M}-\mathrm{H}_{2} \mathrm{O}-\mathrm{CH}_{3}\right]^{+}\right) \\
157\left(95,\left[\mathrm{M}-\mathrm{C}_{4} \mathrm{H}_{9}\right]^{+}\right) \\
129\left(100,\left[\mathrm{M}-\mathrm{C}_{5} \mathrm{H}_{9} \mathrm{O}\right]^{+}\right)\end{array}$ \\
\hline
\end{tabular}

Ratio of 2,2-dimethyl-1-(2-naphthyl)propanol and 2,2-dimethyl-1-(1-naphthyl)propanol, established by ${ }^{1} \mathrm{H}$ NMR was 0.64 to 0.36 , and it was nearly the same as established by GC-MS, which indicated 0.62 to 0.38 ratio.

All isomers of benzonorcaradienes with $t \mathrm{Bu}$ and $i \mathrm{Pr}$ substituents adjacent to cyclopropane ring synthesised form 1- and 2-nitronaphthalene were tested under the same reaction conditions as for synthesis of cycloproparene. Endo-3s gave 5\% of cycloproparene 8 and 95\% substrate recovery 
(confirmed by NMR of crude reaction mixture, mixture was not separated). Endo-3e, exo-3e, exo-3g, endo-3o, exo-3o, and exo-3s gave no product (substrate was recovered).
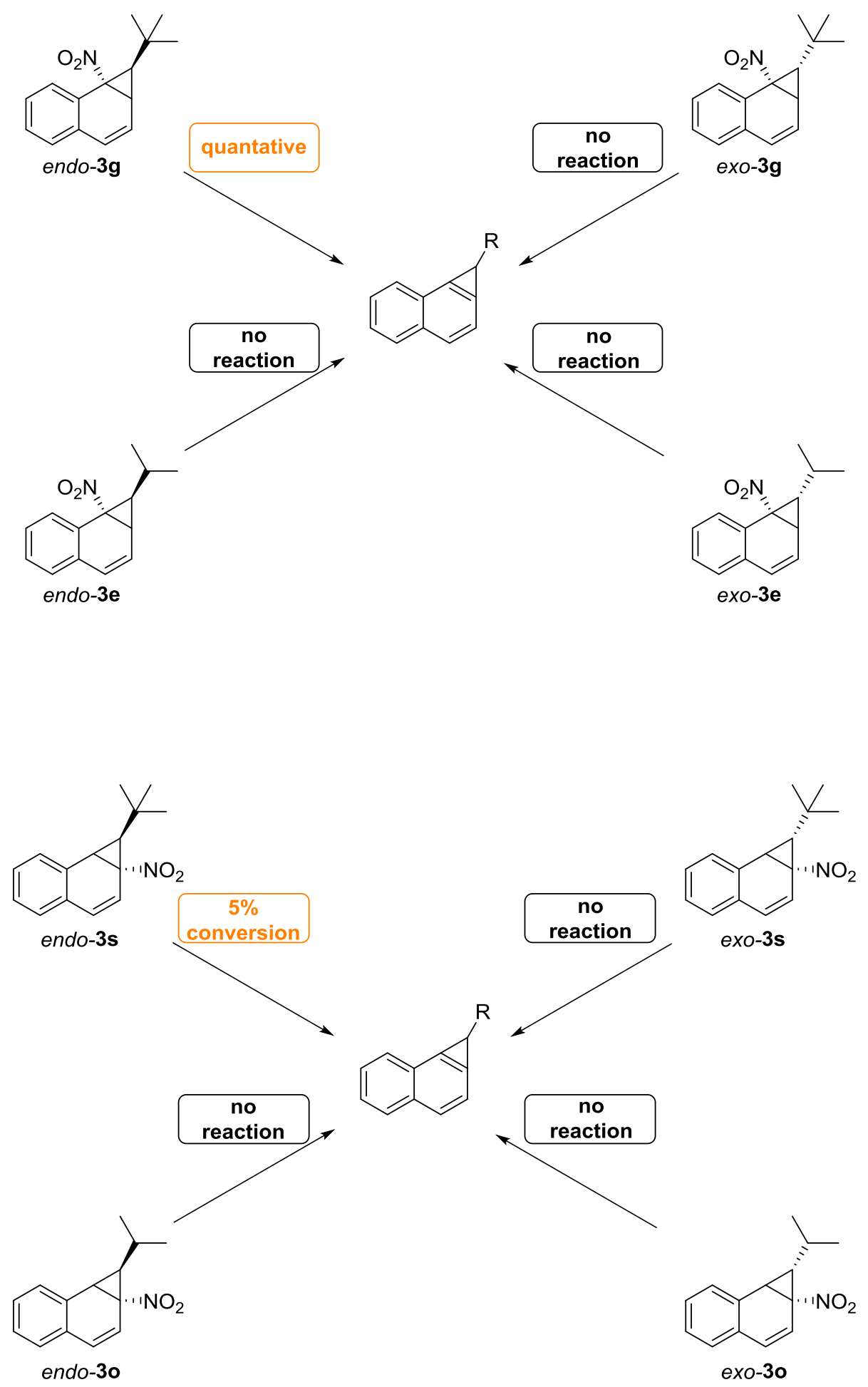


\section{References}

[1] A. Verma, S. Jana, C. D. Prasad, A. Yadav, S. Kumar, Chem. Commun. 2016, 52, 4179-4182.

[2] V. Nascimento, E. E. Alberto, D. W. Tondo, D. Dambrowski, M. R. Detty, F. Nome, A. L. Braga, J. Am. Chem. Soc. 2012, 134, 138-141.

[3] S. Potash, S. Rozen, European J. Org. Chem. 2013, 2013, 5574-5579.

[4] M. Adamczyk, Y. Y. Chen, P. G. Mattingly, Y. Pan, S. Rege, J. Org. Chem. 1998, 63, 5636-5639.

[5] H. Matsuyama, T. Nakamura, M. Iyoda, J. Org. Chem. 2000, 65, 4796-4803.

[6] M. Lozano González, L. Bousquet, S. Hameury, C. Alvarez Toledano, N. Saffon-Merceron, V. Branchadell, E. Maerten, A. Baceiredo, Chem. Eur. J. 2018, 24, 2570-2574.

[7] T. J. Wenzel, J. Zaia, Anal. Chem. 1987, 59, 562-567.

[8] M. Mischitz, C. Mirtl, R. Saf, K. Faber, Tetrahedron Asymmetry 1996, 7, 2041-2046.

[9] X. Gao, Y. Zhang, B. Wang, New J. Chem. 2005, 29, 579-586.

[10] H. Maehr, J. Smallheer, J. Am. Chem. Soc. 1985, 107, 2943-2945.

[11] O. Tamura, T. Takeya, Y. Takahashi, I. Okamoto, Heterocycles 2010, 80, 1479-1488.

[12] M. Makosza, Z. Owczarczyk, J. Org. Chem. 1989, 54, 5094-5100.

[13] M. Chakrabarty, T. Kundu, S. Arima, Y. Harigaya, Tetrahedron 2008, 64, 6711-6723.

[14] Y. Wang, G. Zhang, G. Hu, Y. Bu, H. Lei, D. Zuo, M. Han, X. Zhai, P. Gong, Eur. J. Med. Chem. 2016, 123, 80-89.

[15] B. Zhang, S. Li, S. Yue, M. Cokoja, M. D. Zhou, S. L. Zang, F. E. Kühn, J. Organomet. Chem. 2013, 744, 108-112.

[16] M. Achmatowicz, O. R. Thiel, G. Gorins, C. Goldstein, C. Affouard, R. Jensen, R. D. Larsen, J. Org. Chem. 2008, 73, 6793-6799.

[17] D. Ströhl, S. Thomas, E. Kleinpeter,R. Radeglia, J. Brunn, Monatsh Chem. 1992, 123, 769-777.

[18] V. J. Traynelis, J. V. McSweeney, J. Org. Chem. 1966, 31, 243-247.

[19] S. Bühler, I. Lagoja, H. Giegrich, K. P. Stengele, W. Pfleiderer, Helv. Chim. Acta 2004, 87, 620-659.

[20] R. G. R. Bacon, R. G. Guy, R. S. Irwin, J. Chem. Soc. 1961, 2436-2447.

[21] K. Suzawa, M. Ueno, A. E. H. Wheatley, Y. Kondo, Chem. Commun. 2006, 4850-4852.

[22] P. Stanetty, H. Wallner, Arch. Pharm. (Weinheim) 1993, 326, 341-350. 
9. Reproductions of ${ }^{1} \mathrm{H}$ and ${ }^{13} \mathrm{C}$ NMR spectra<smiles>C[Se](=O)(=O)c1ccccc1</smiles>

$2 a$

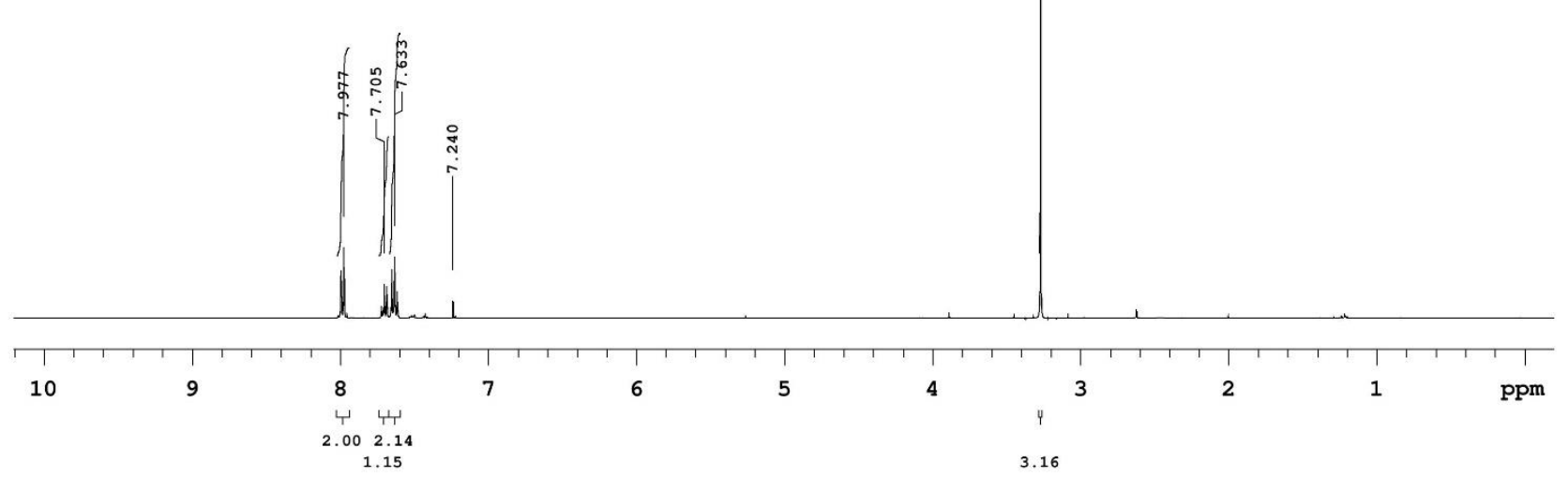

Sample Name: TB-094

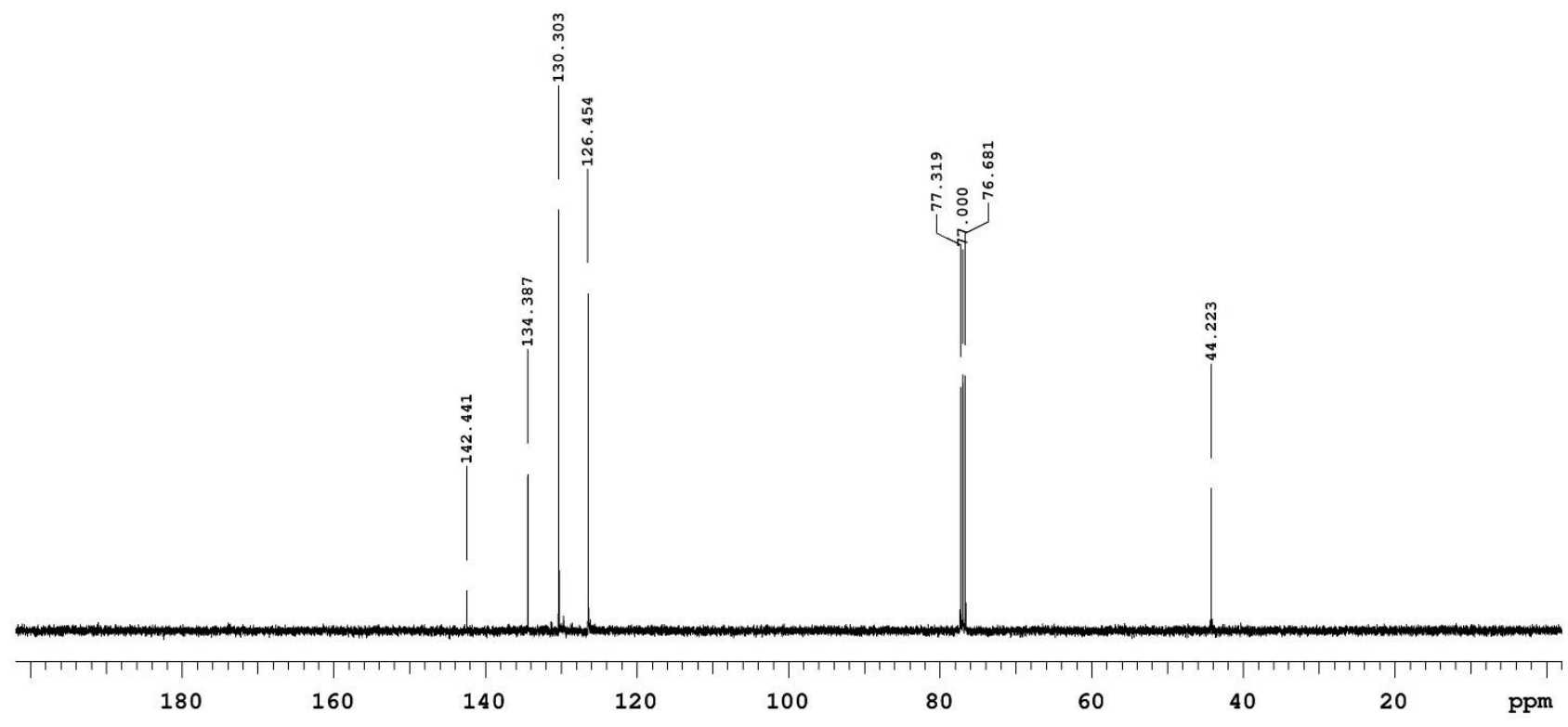

S-79 
Sample Name: TB-155

Solvent: cdc13

$N^{\text {Sen }}$

$2 b$

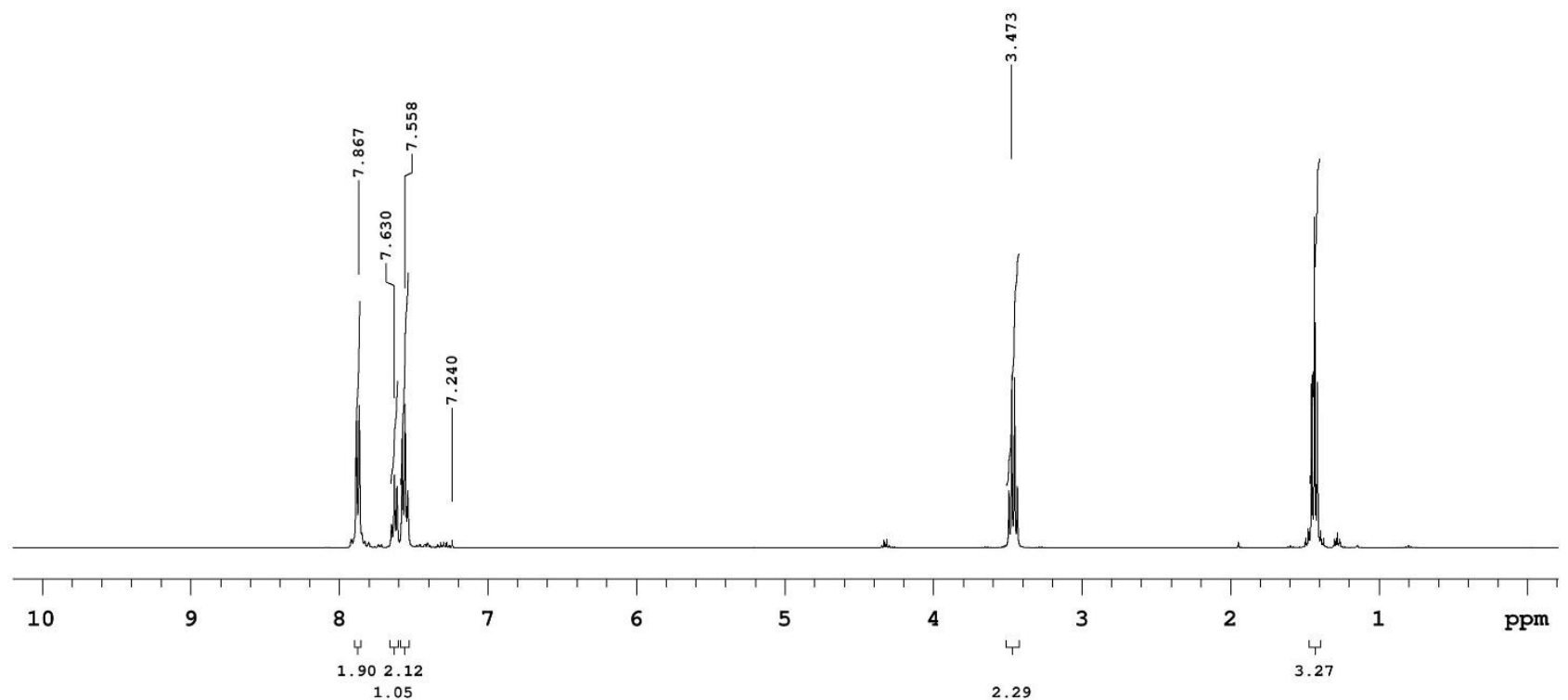

Sample Name: TB-155

Solvent: cdc13

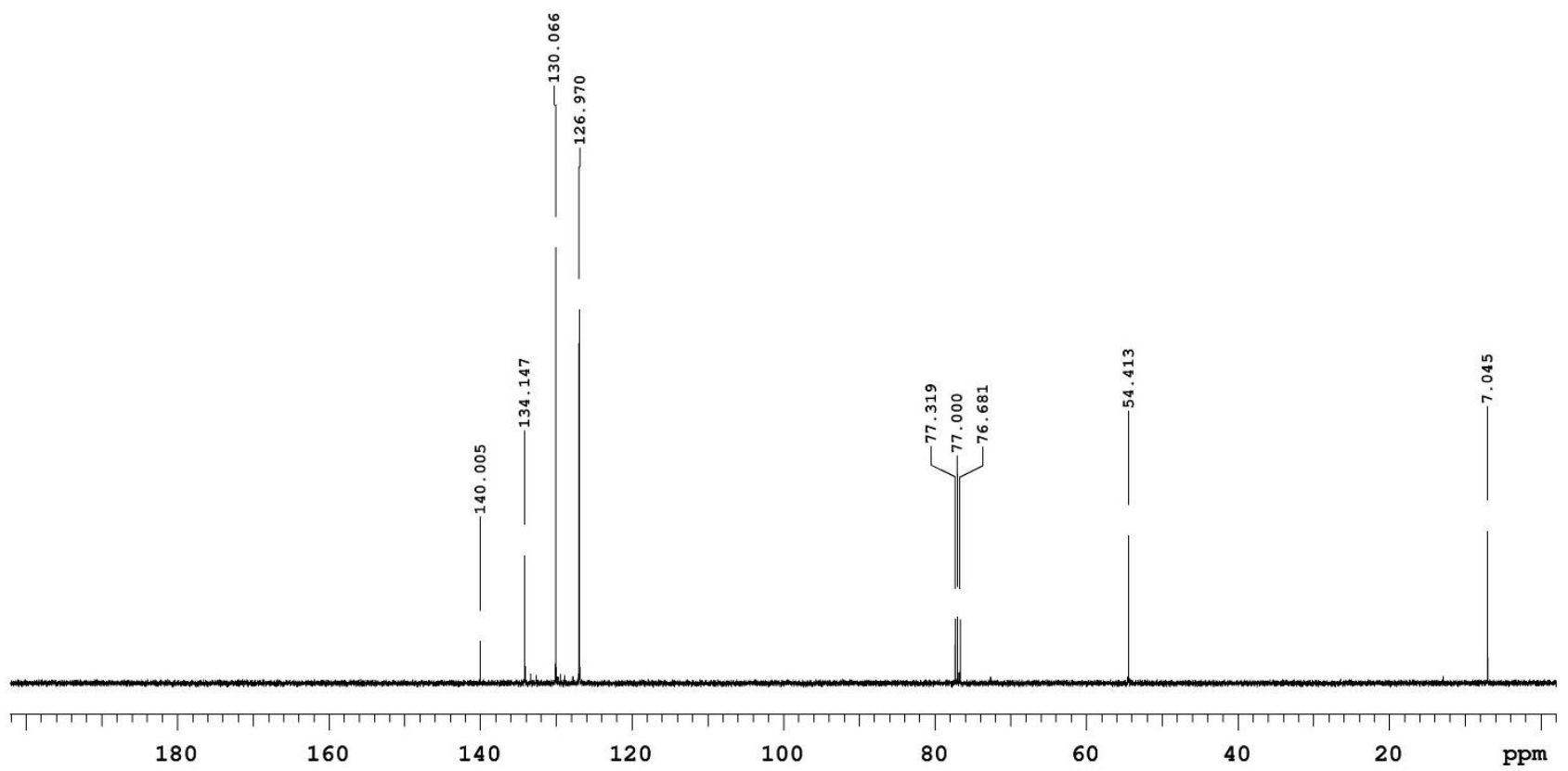



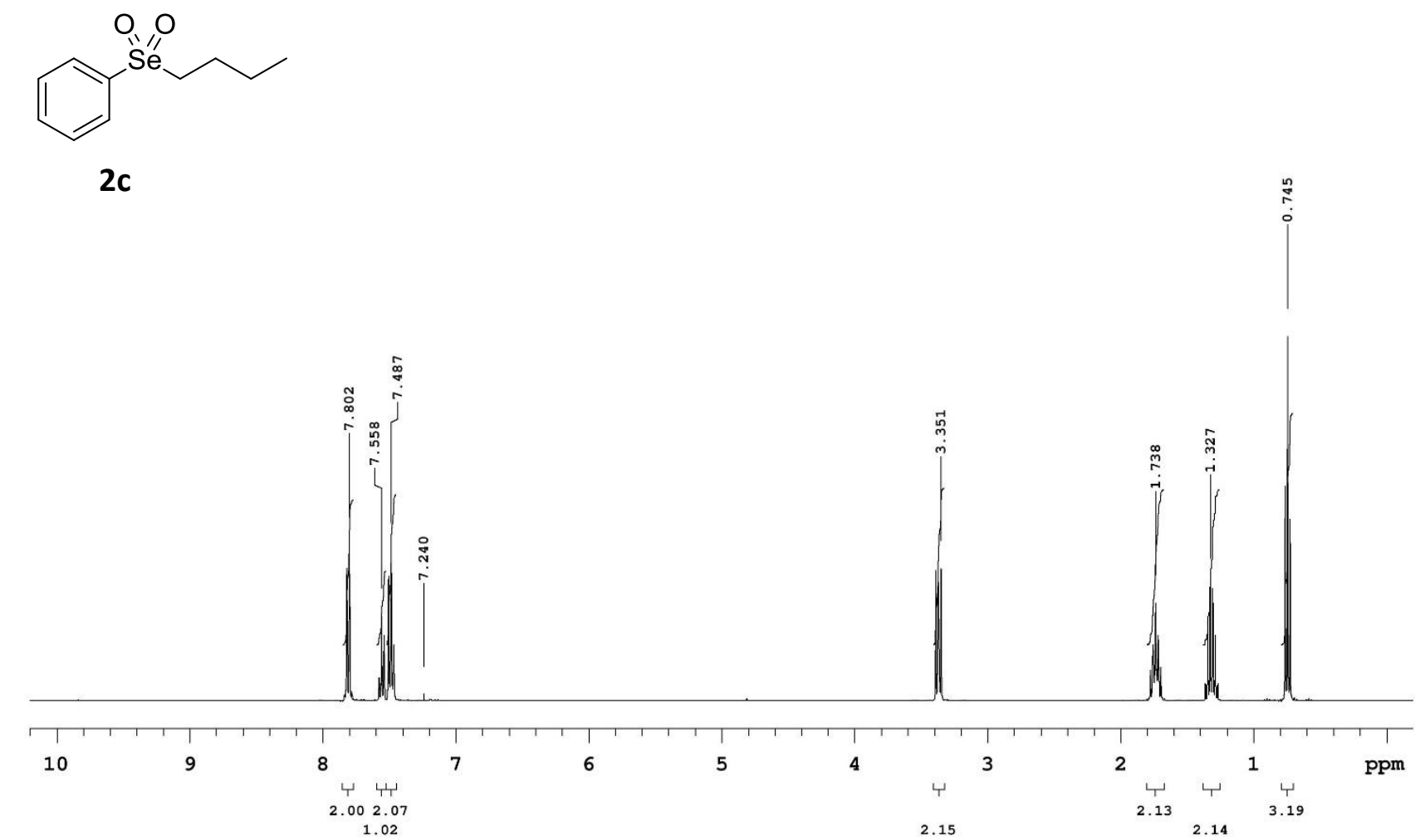

Sample Name: BP-038C

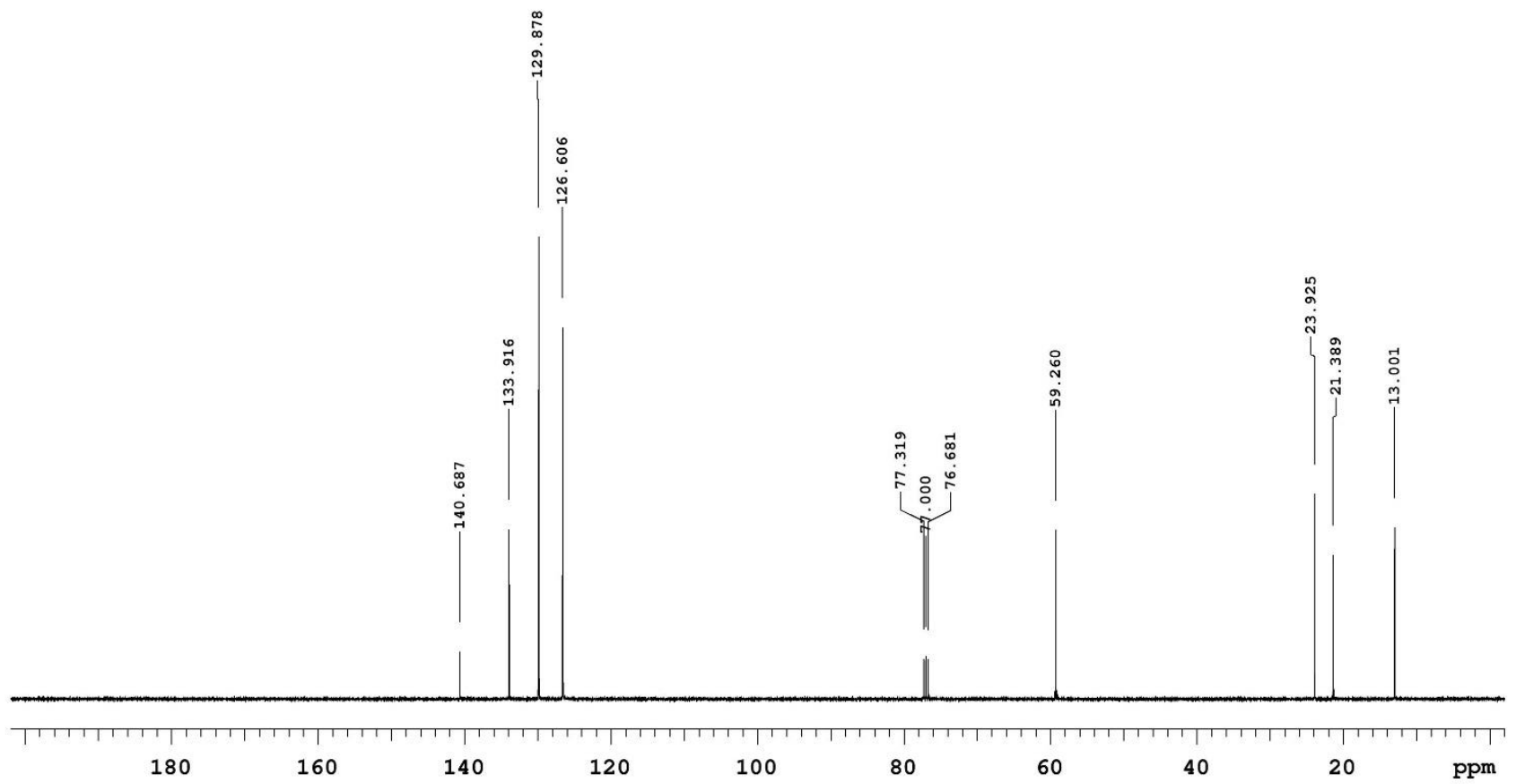




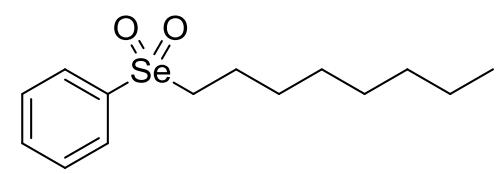

2d

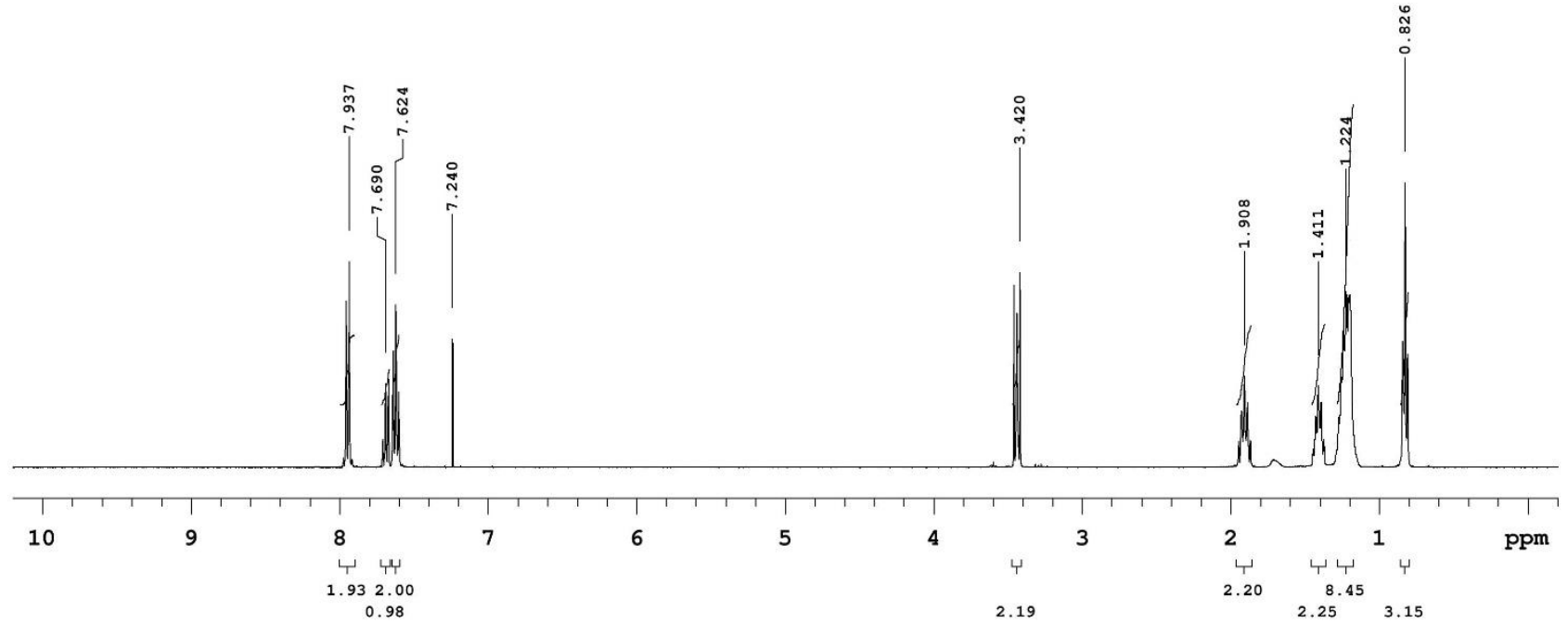

Sample Name: BP-026prod Solvent: $\operatorname{cdcl} 3$

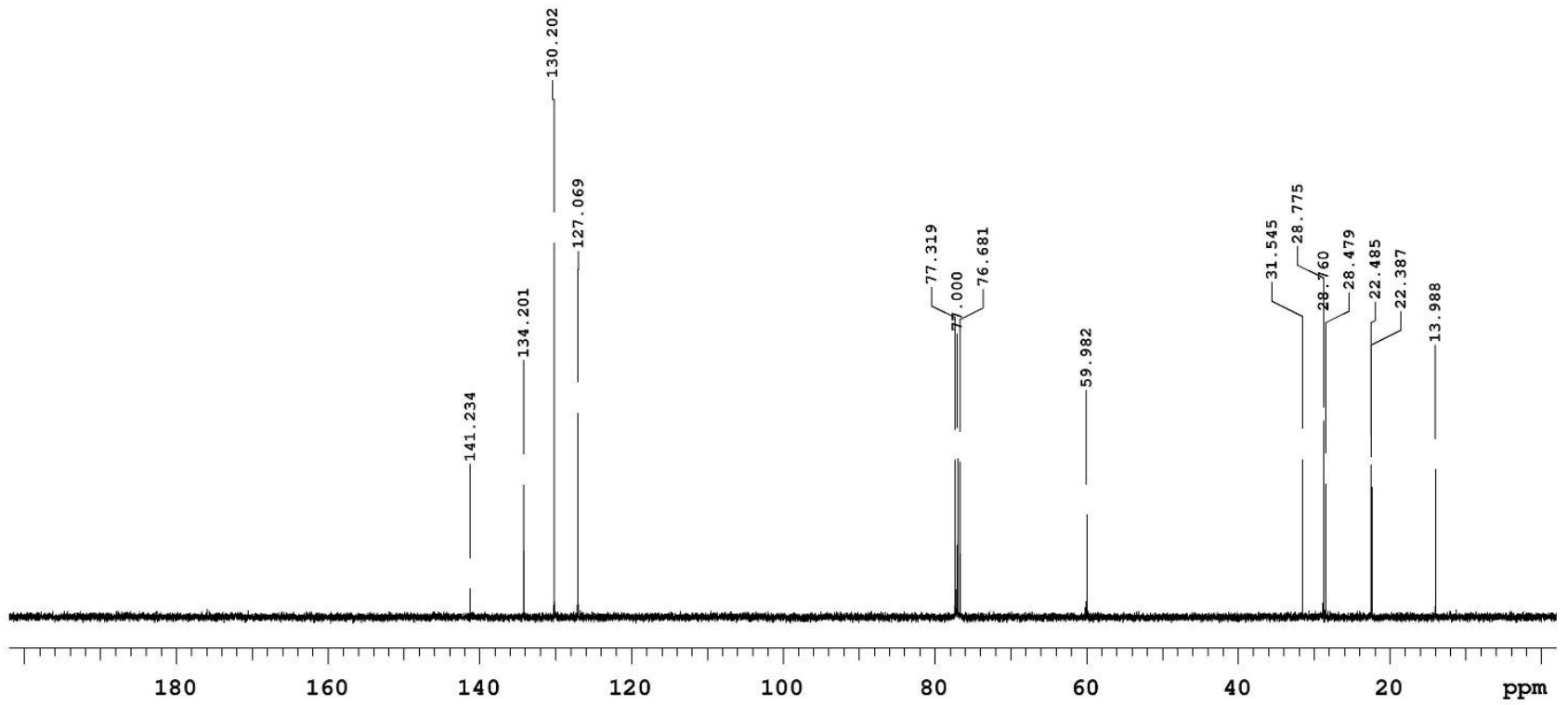


Sample Name: BP-024_ut1_kol

Solvent: cdc13

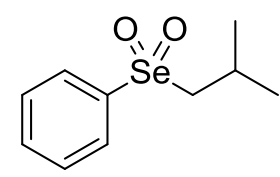

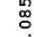

$2 e$

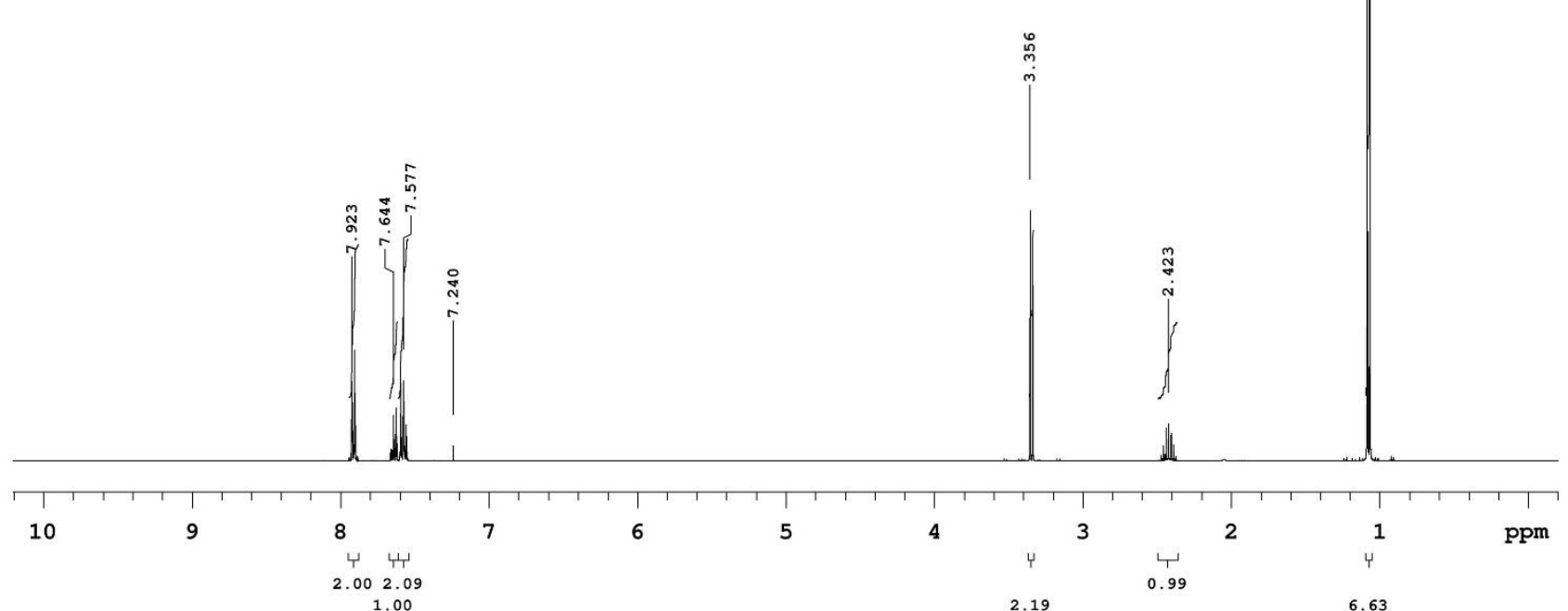

Sample Name: BP-024_ut1_kol Solvent: cdc13

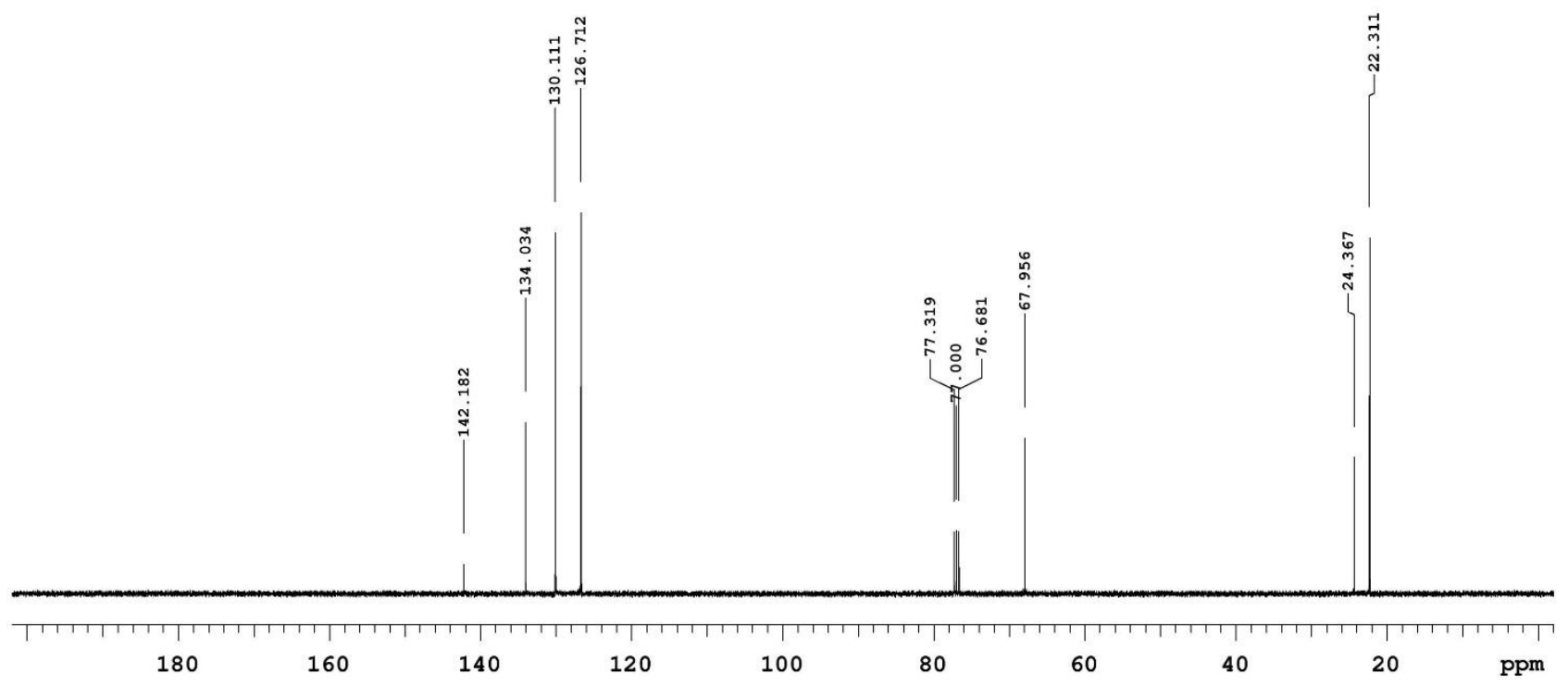


Sample Name: TB-216

Solvent: cdc13

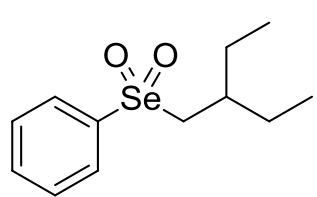

$2 f$
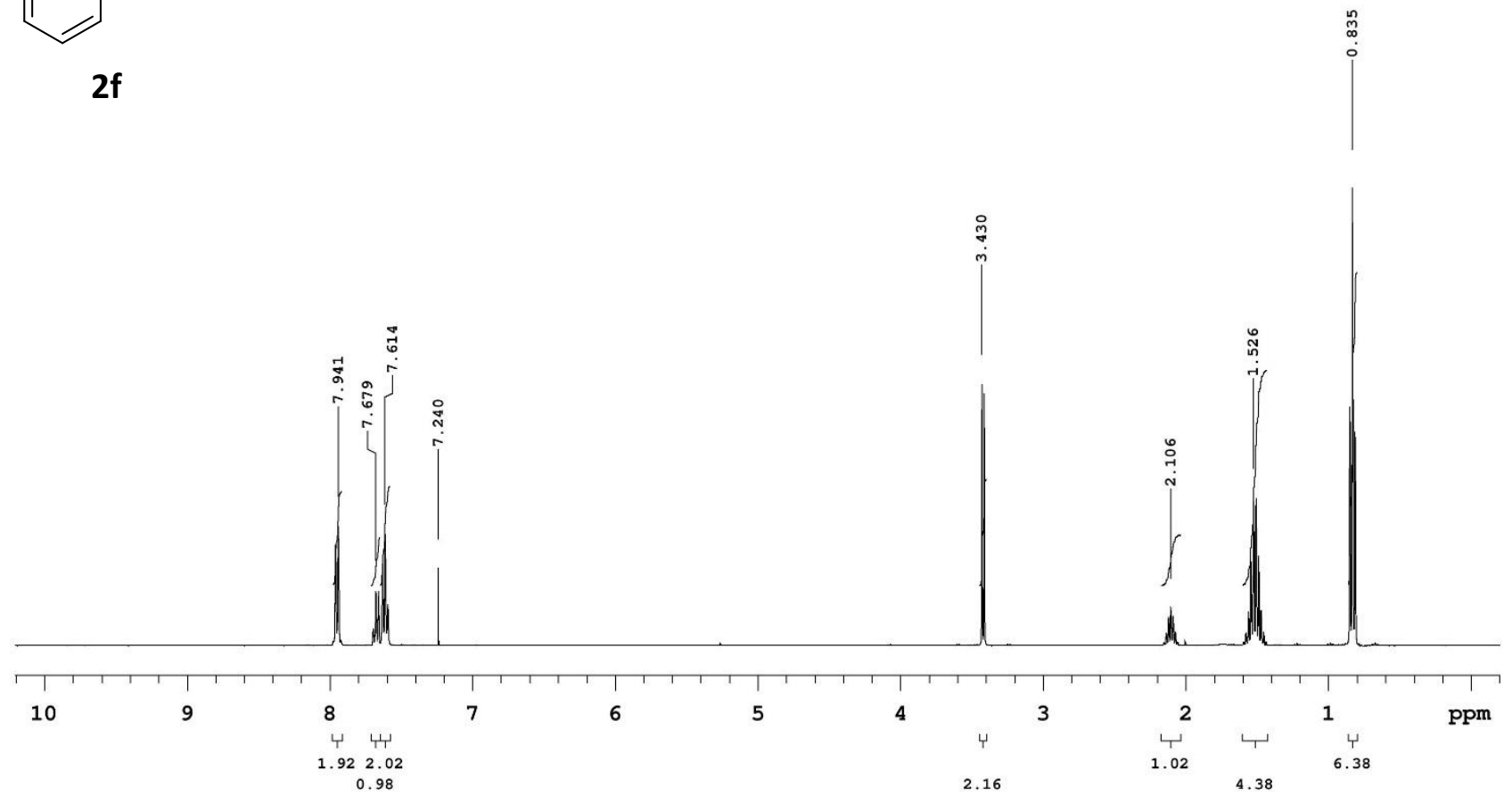

Sample Name: TB-216

Solvent: cdcl3

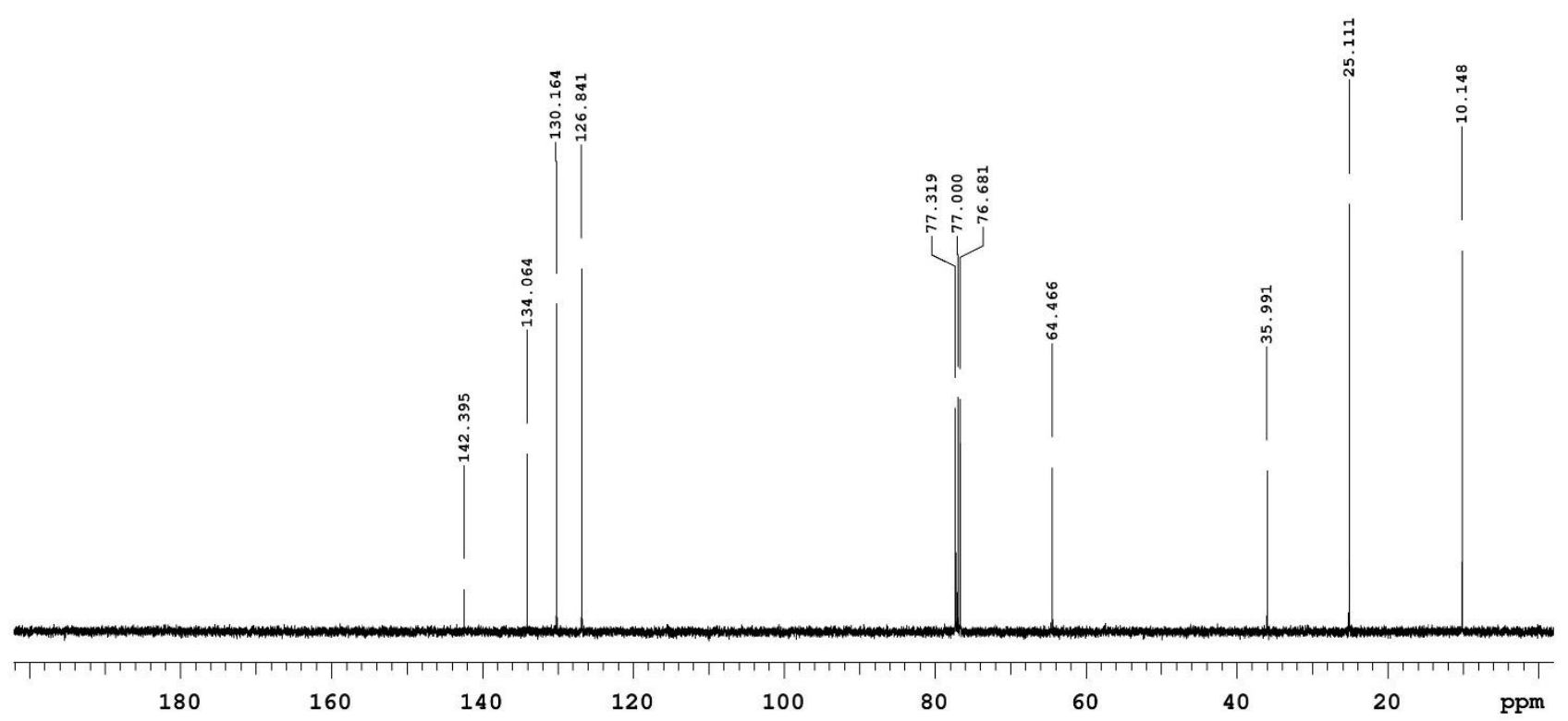



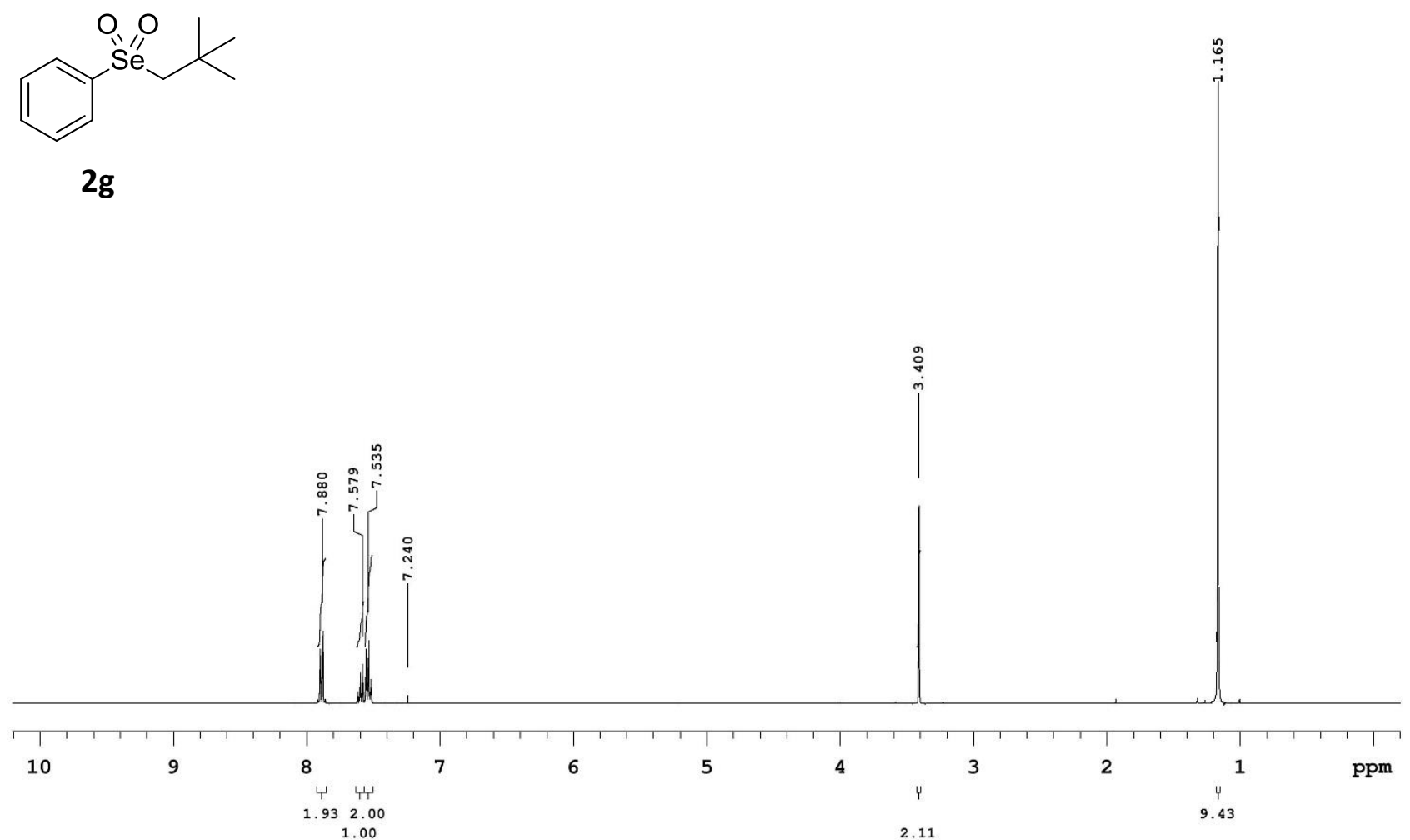

Sample Name: BP-039b Solvent: cdc13

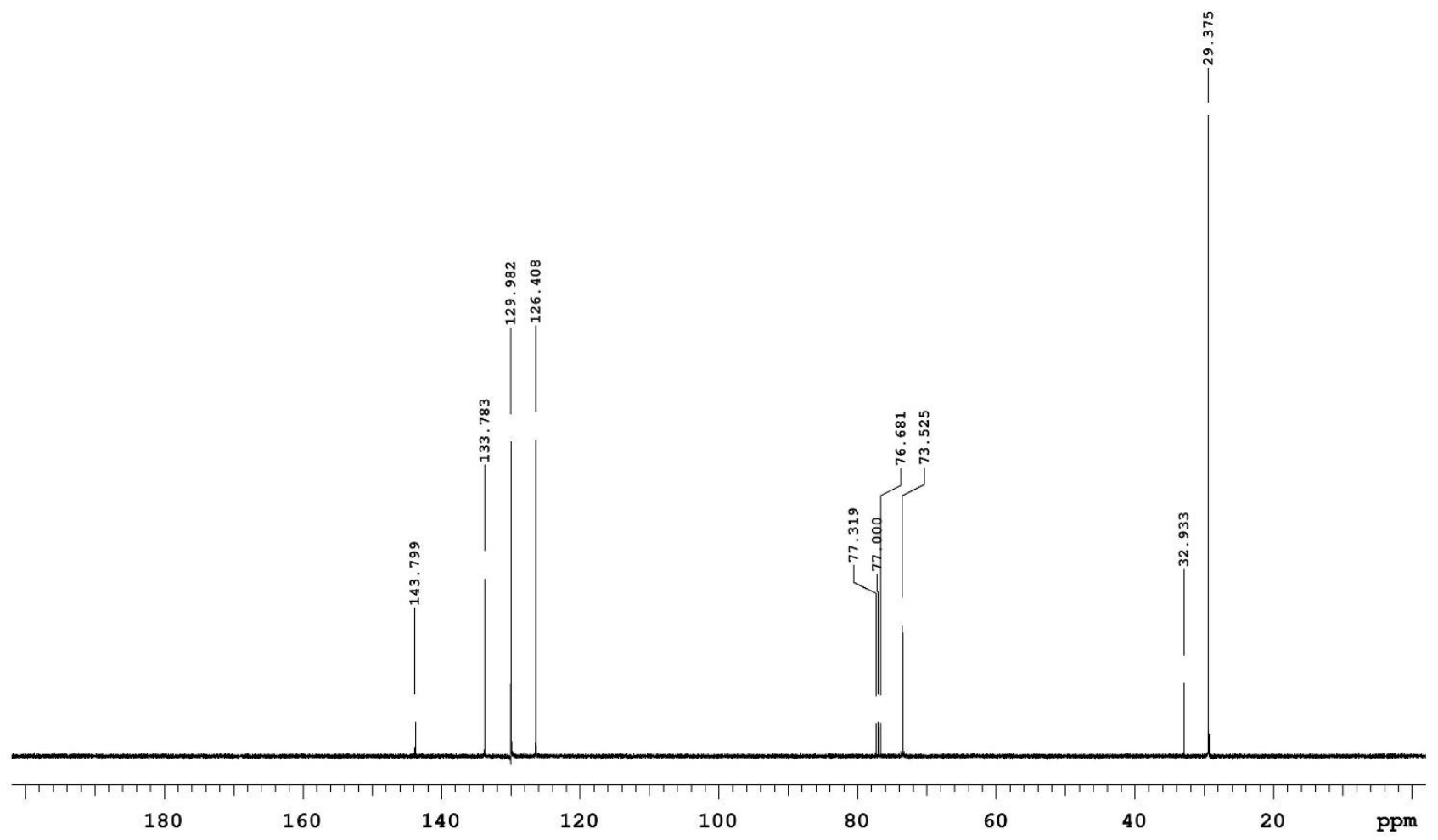



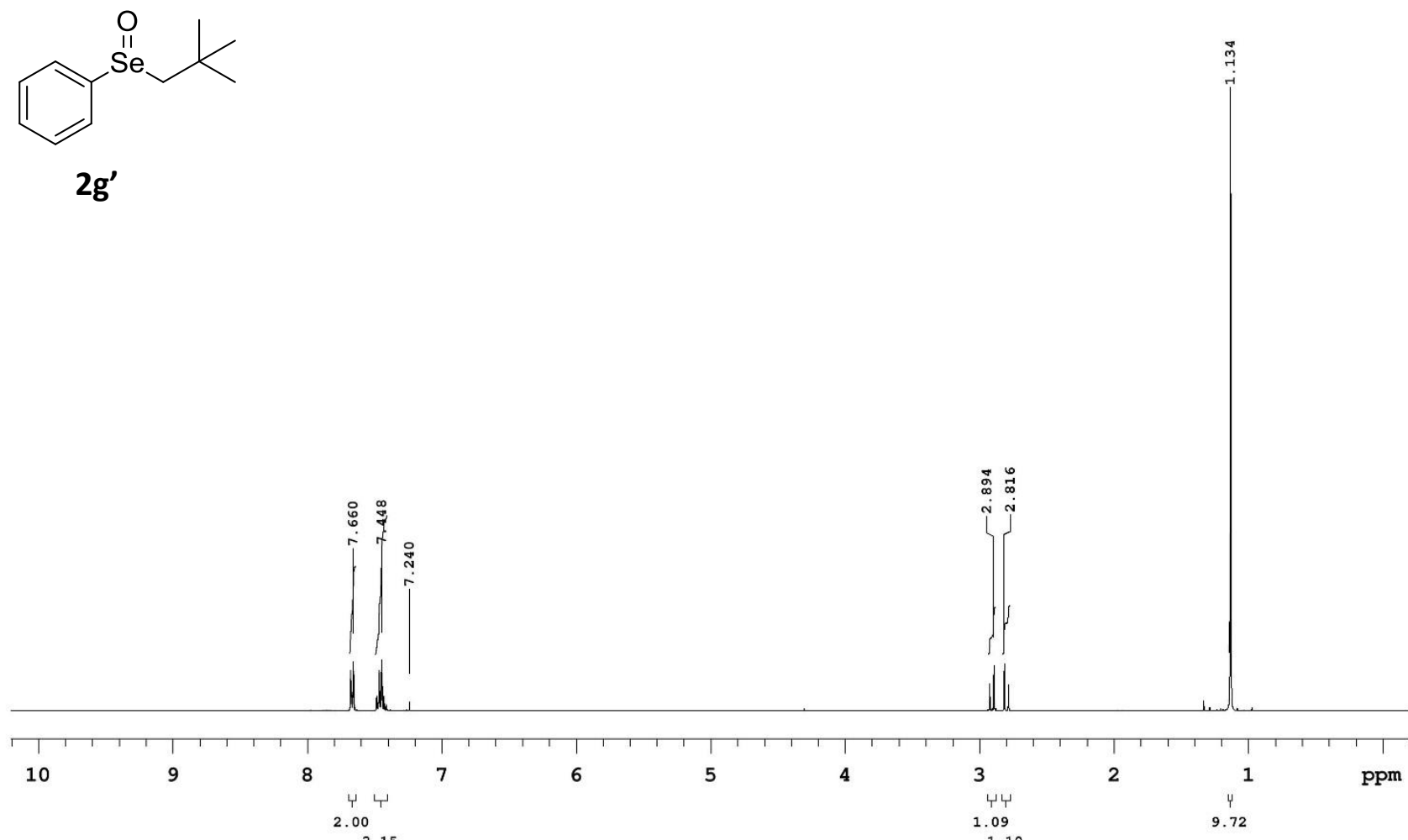

Sample Name: BP-039c suszone Solvent: cdc13

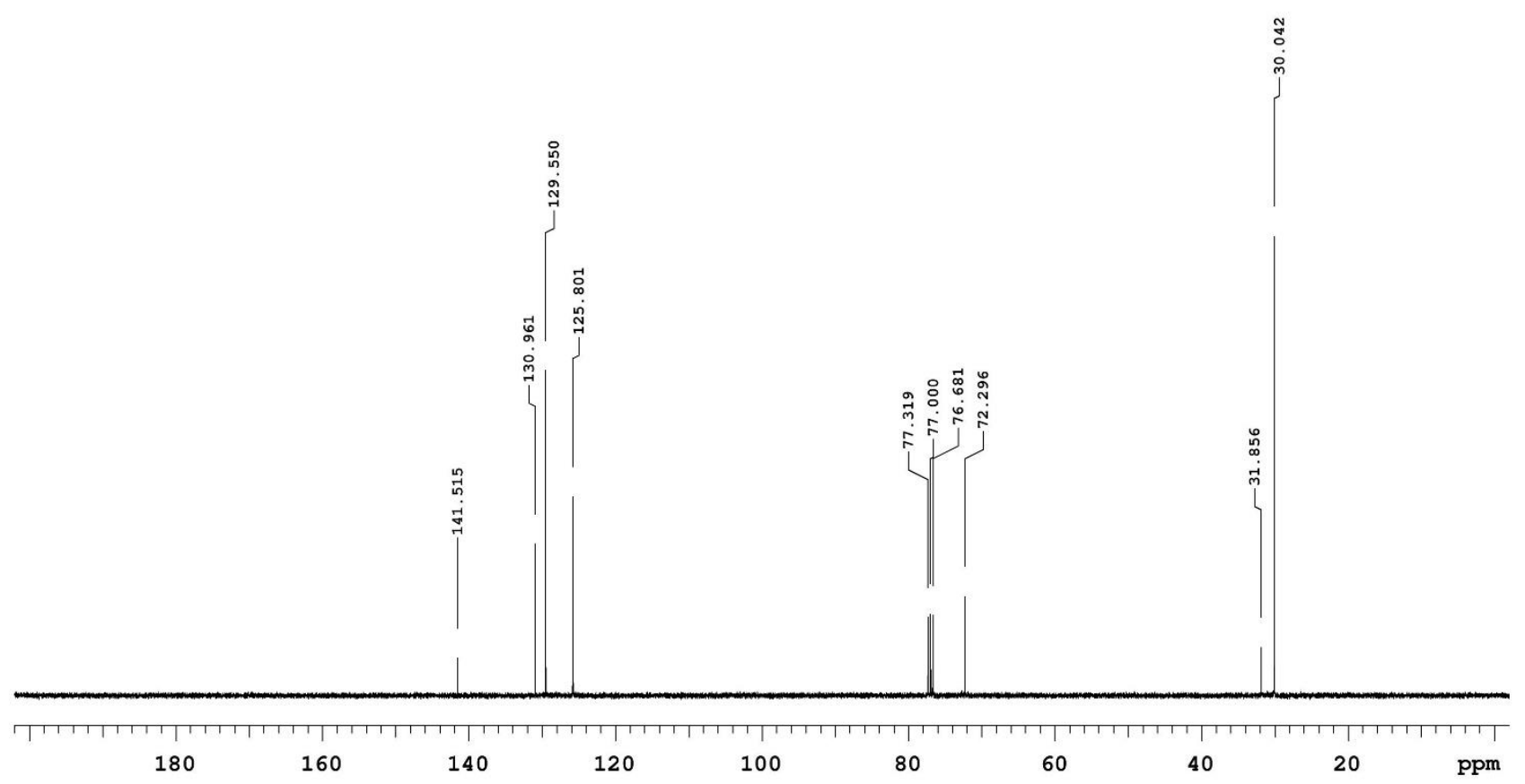


Sample Name: TB-218

Solvent: cdc13

Sis

$2 \mathrm{~h}$

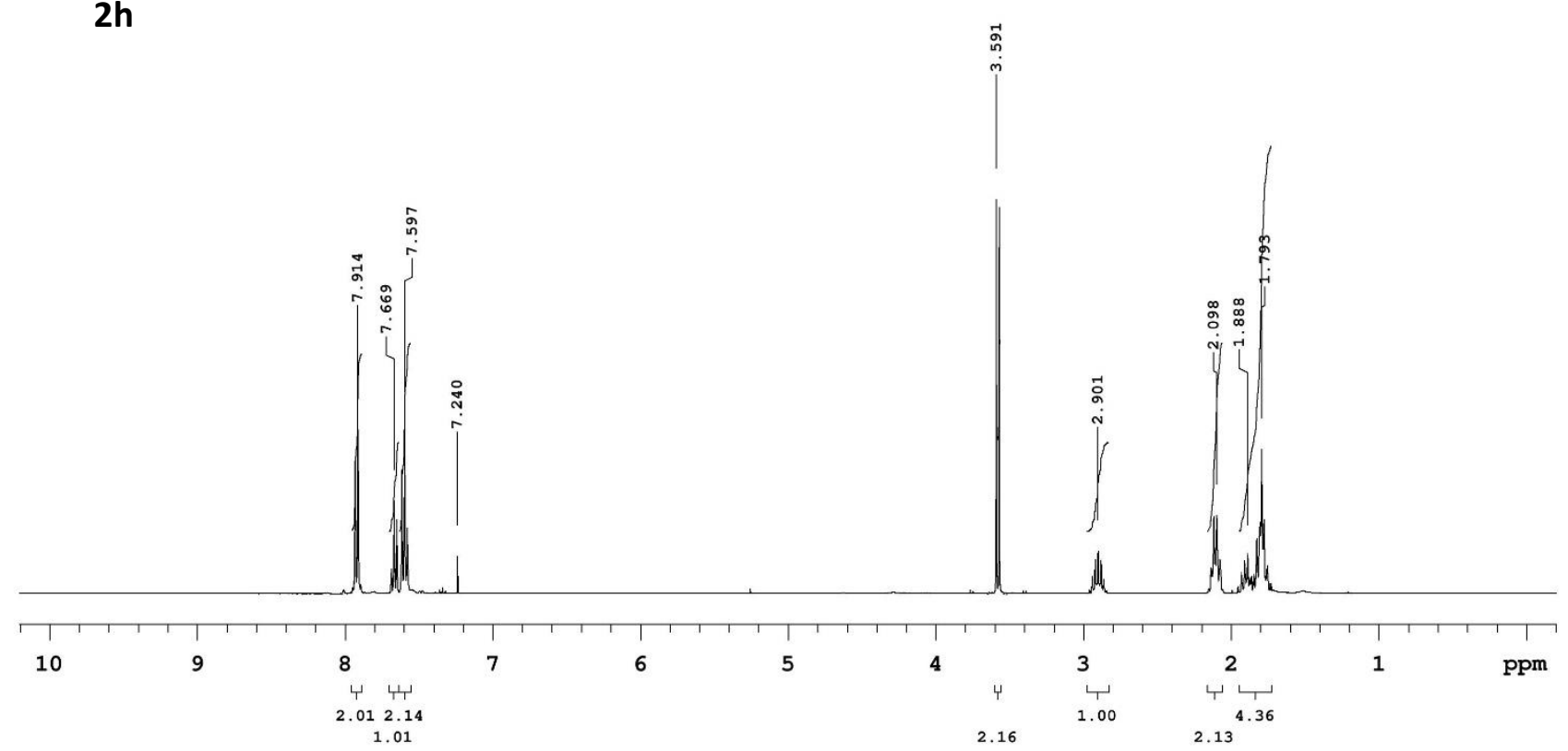

Sample Name: TB-218

Solvent: cdc13

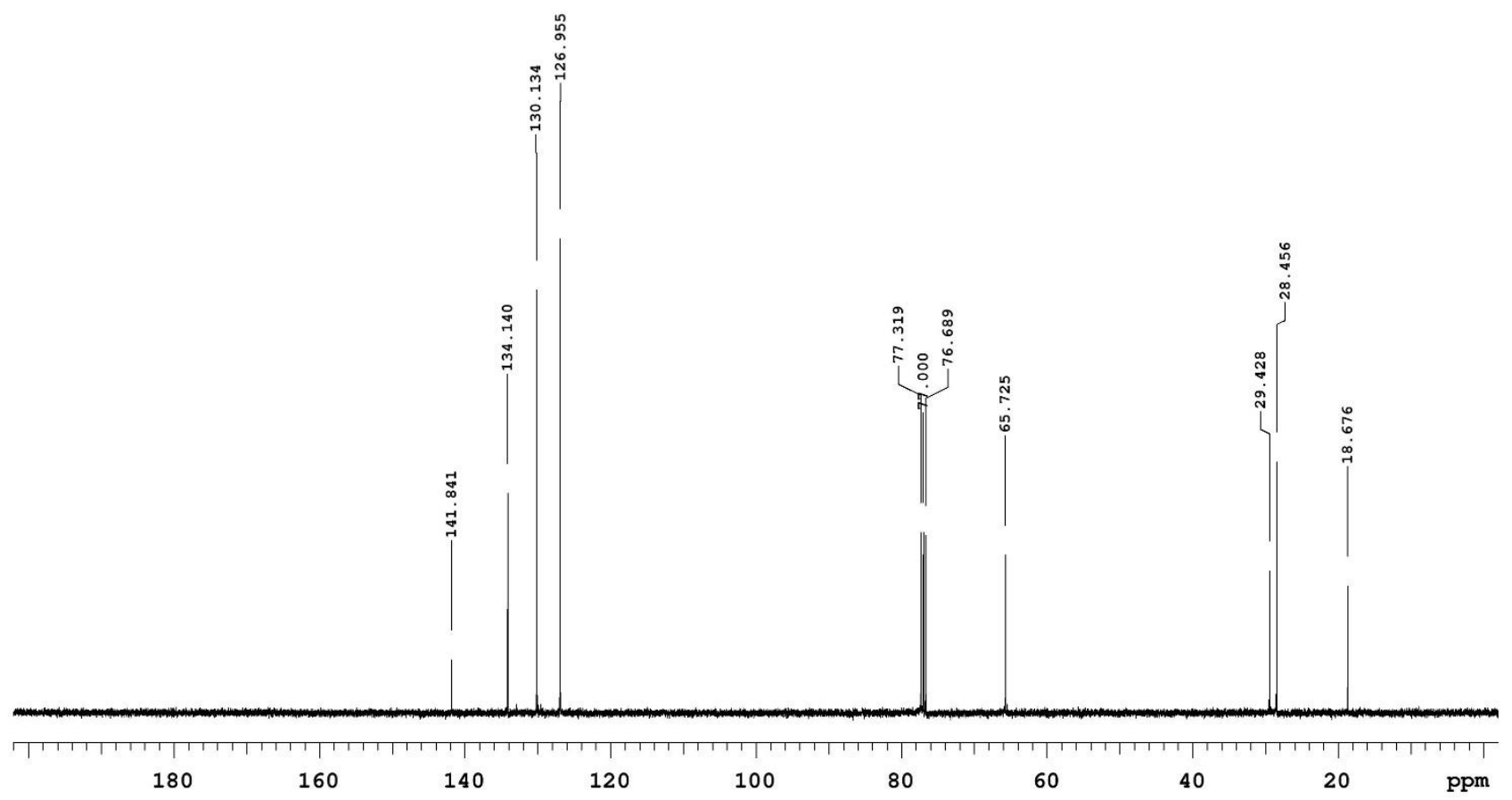



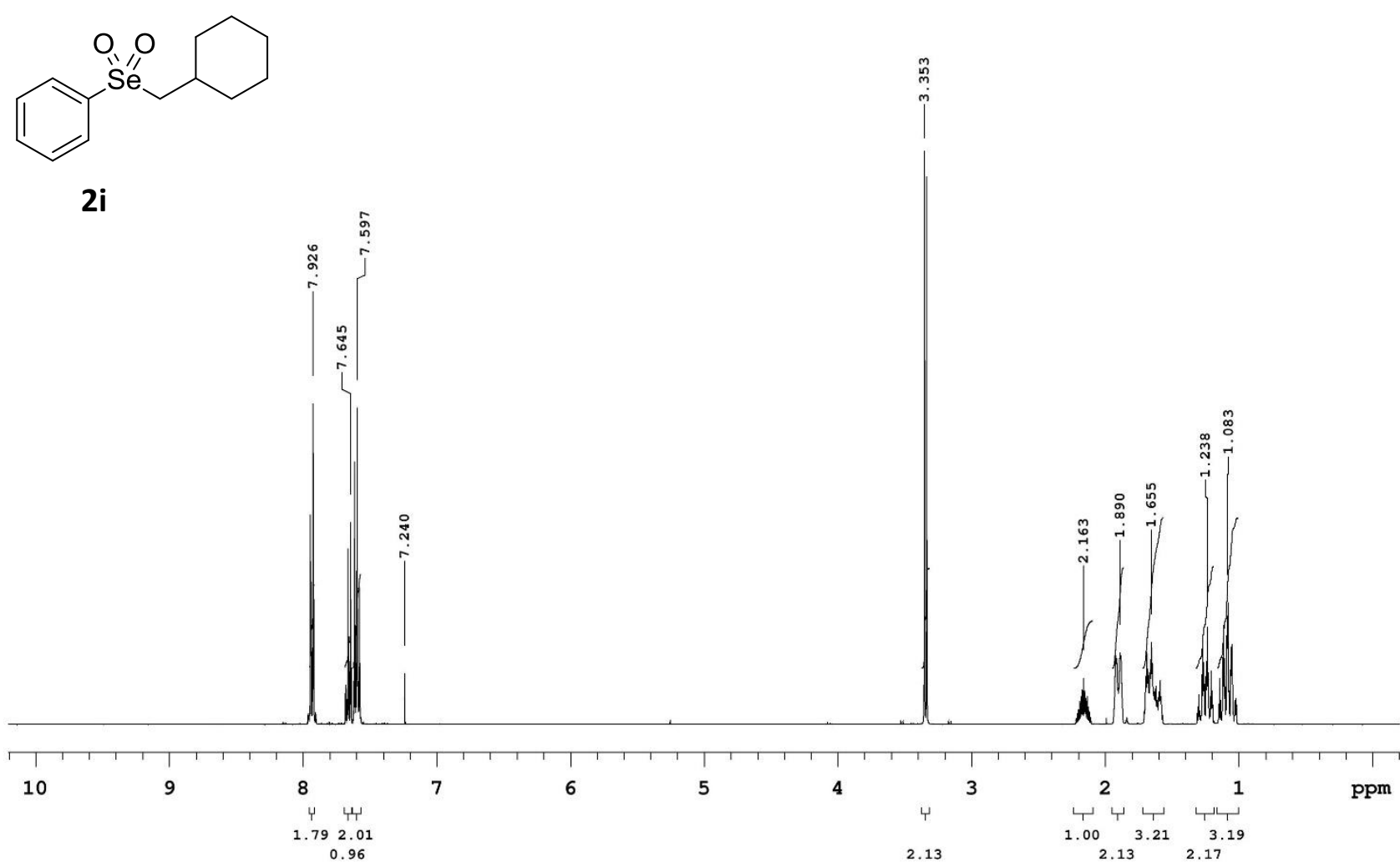

Sample Name: BP-0440st

Solvent: cdcl3

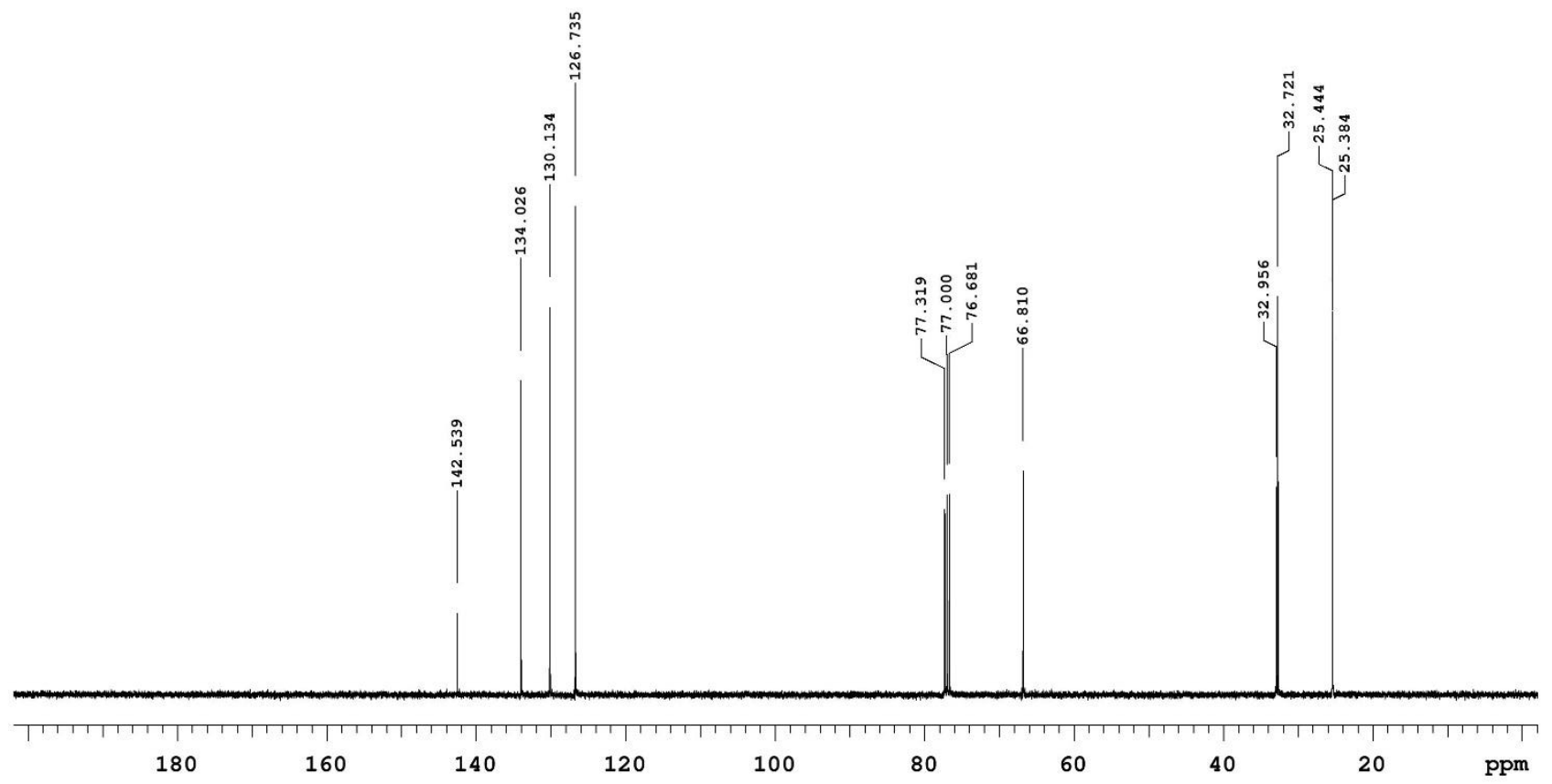


Sample Name: DA-200

Solvent: $\operatorname{cdc13}$

$\overbrace{1}^{\ominus}$

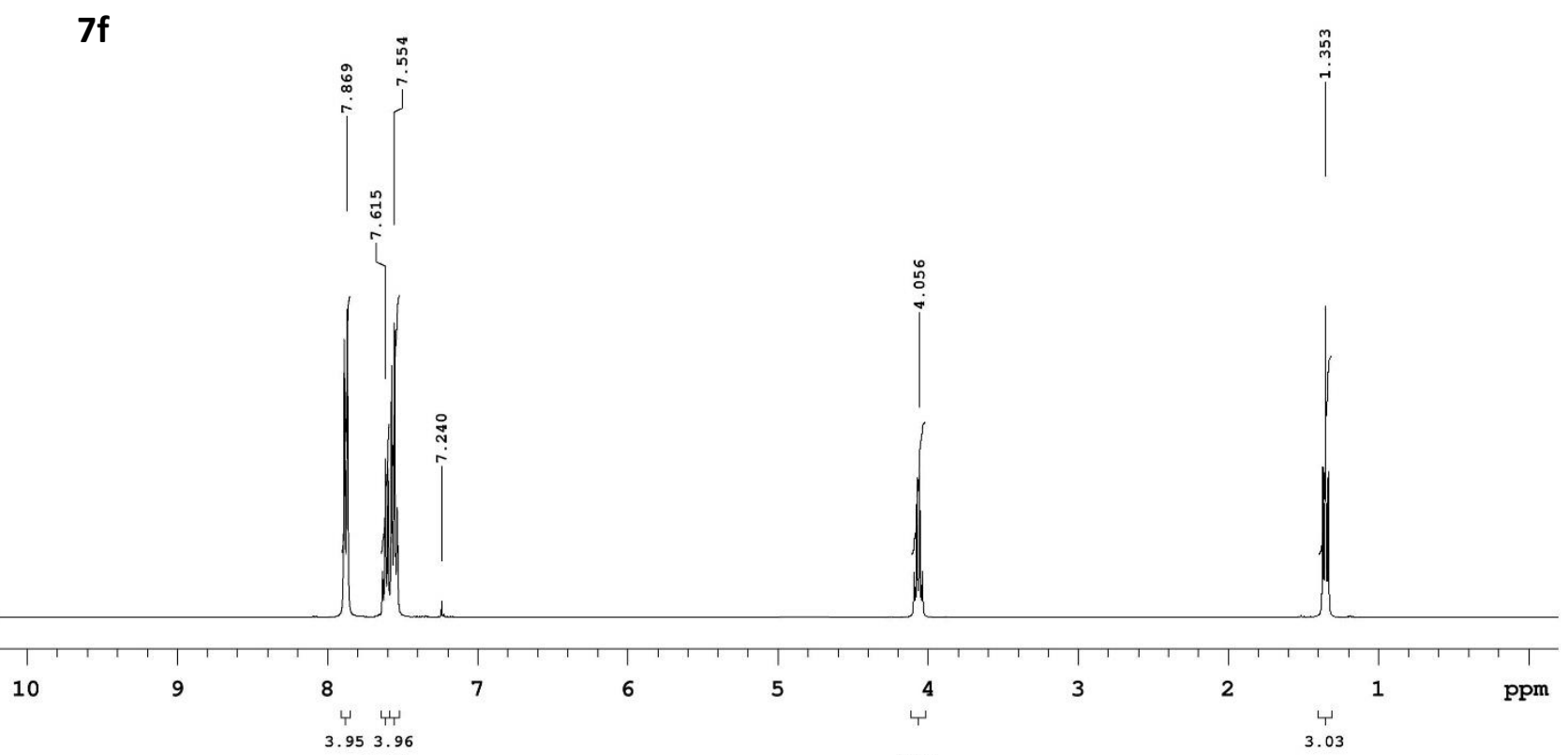

Sample Name: DA-200

Solvent: cdc13

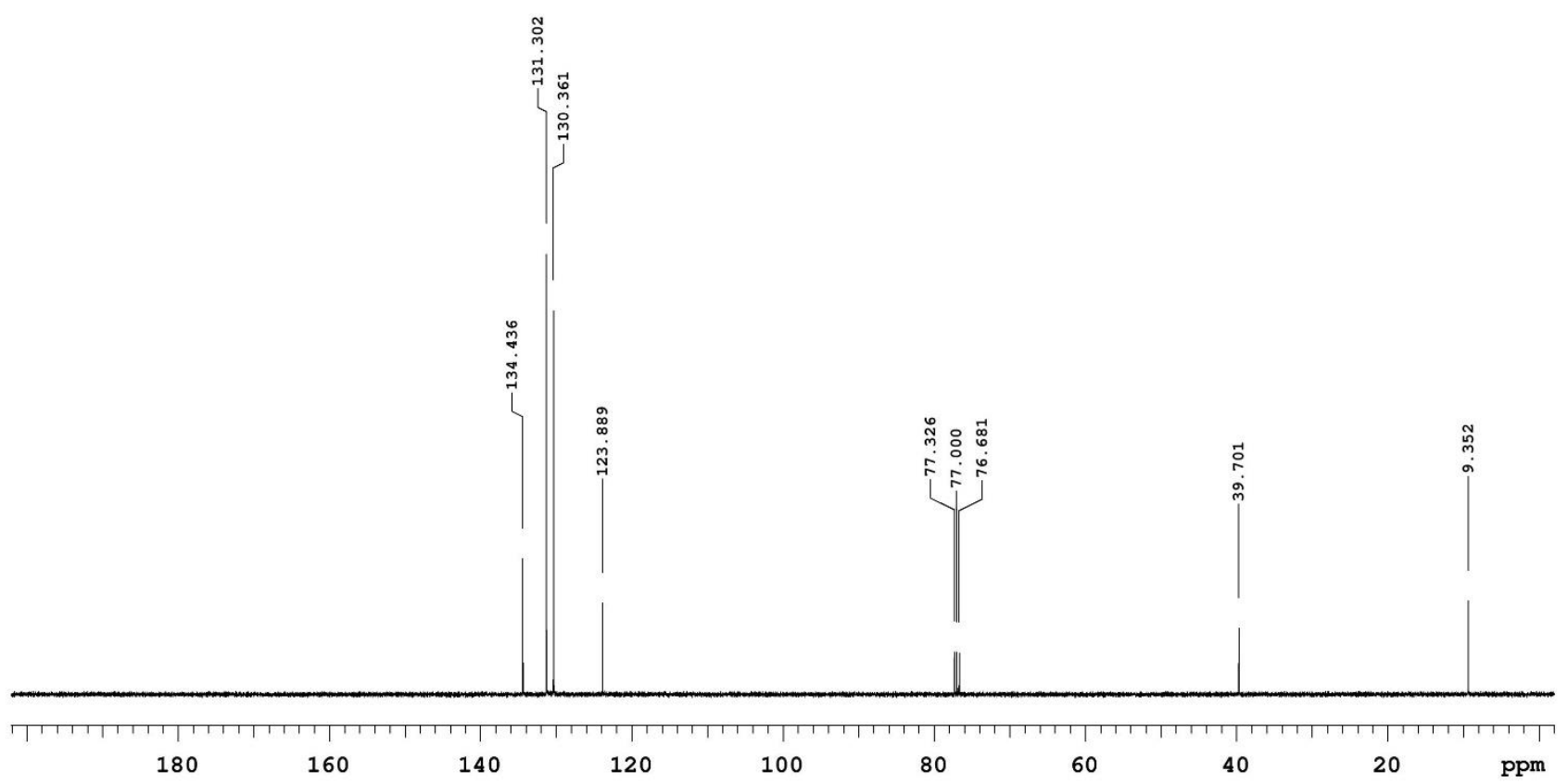


Sample Name: DA-201bb

Solvent: cdc13<smiles>CC[Sb](=O)(c1ccccc1)c1ccccc1Br</smiles>

$7 e$

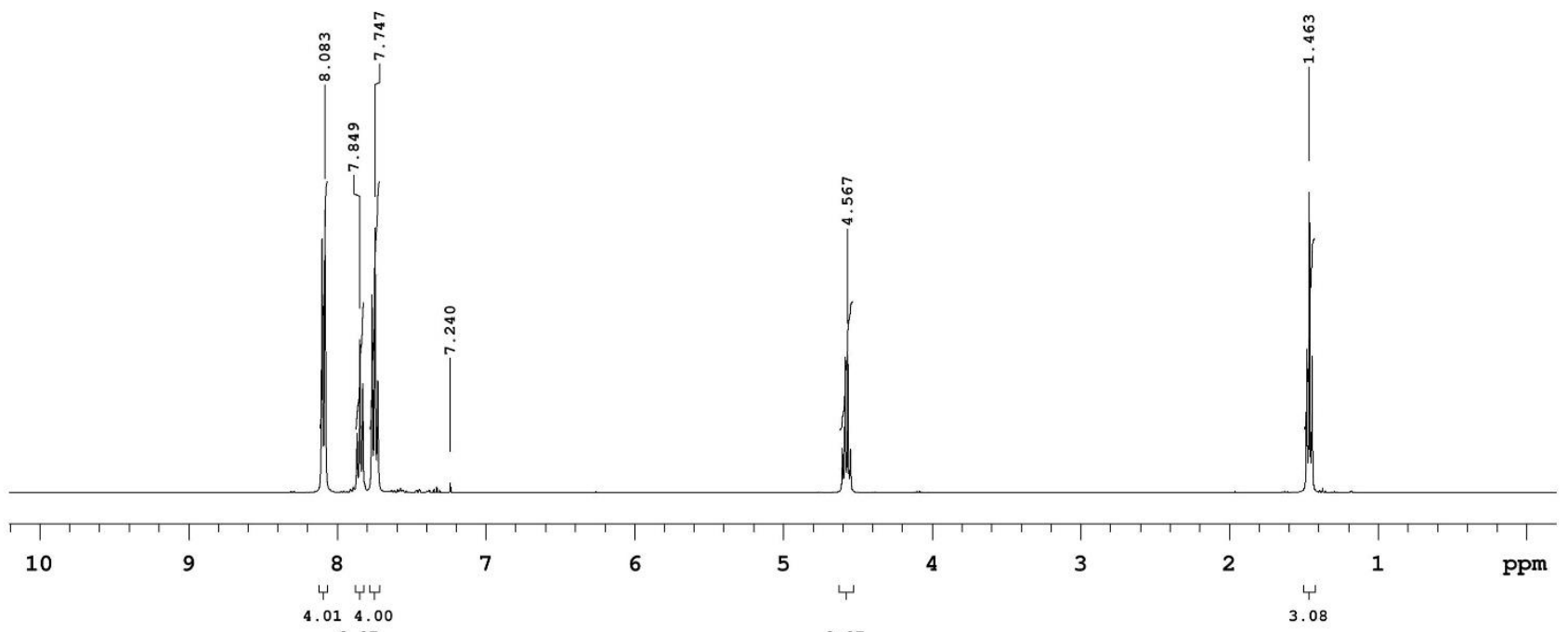

Sample Name: DA-201bb

Solvent: $\operatorname{cdcl} 3$

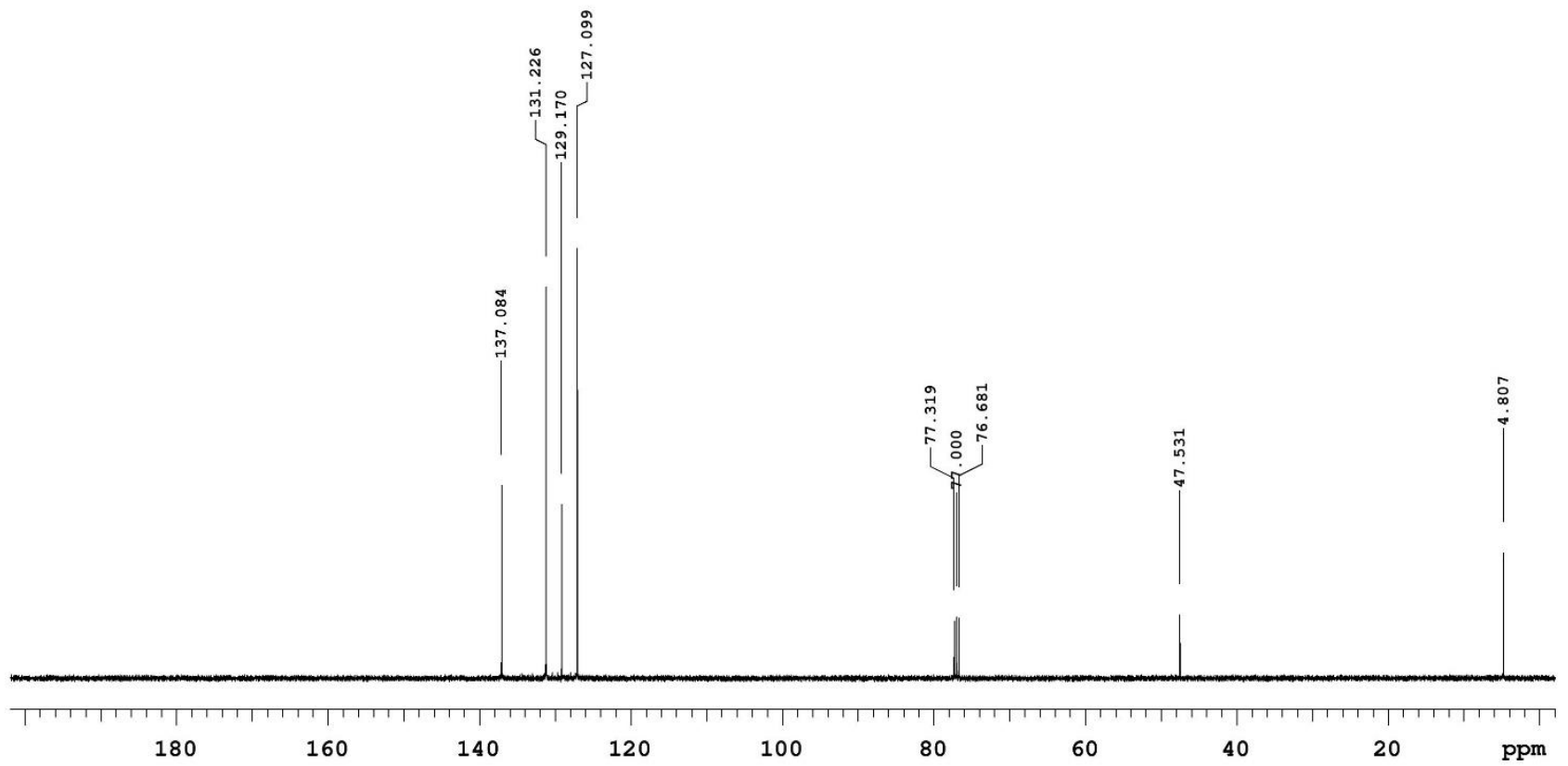


Sample Name: DA-240

Solvent: cdc13<smiles>CC(C)[S+](c1ccccc1)c1ccccc1Br</smiles>

$7 \mathrm{~g}$

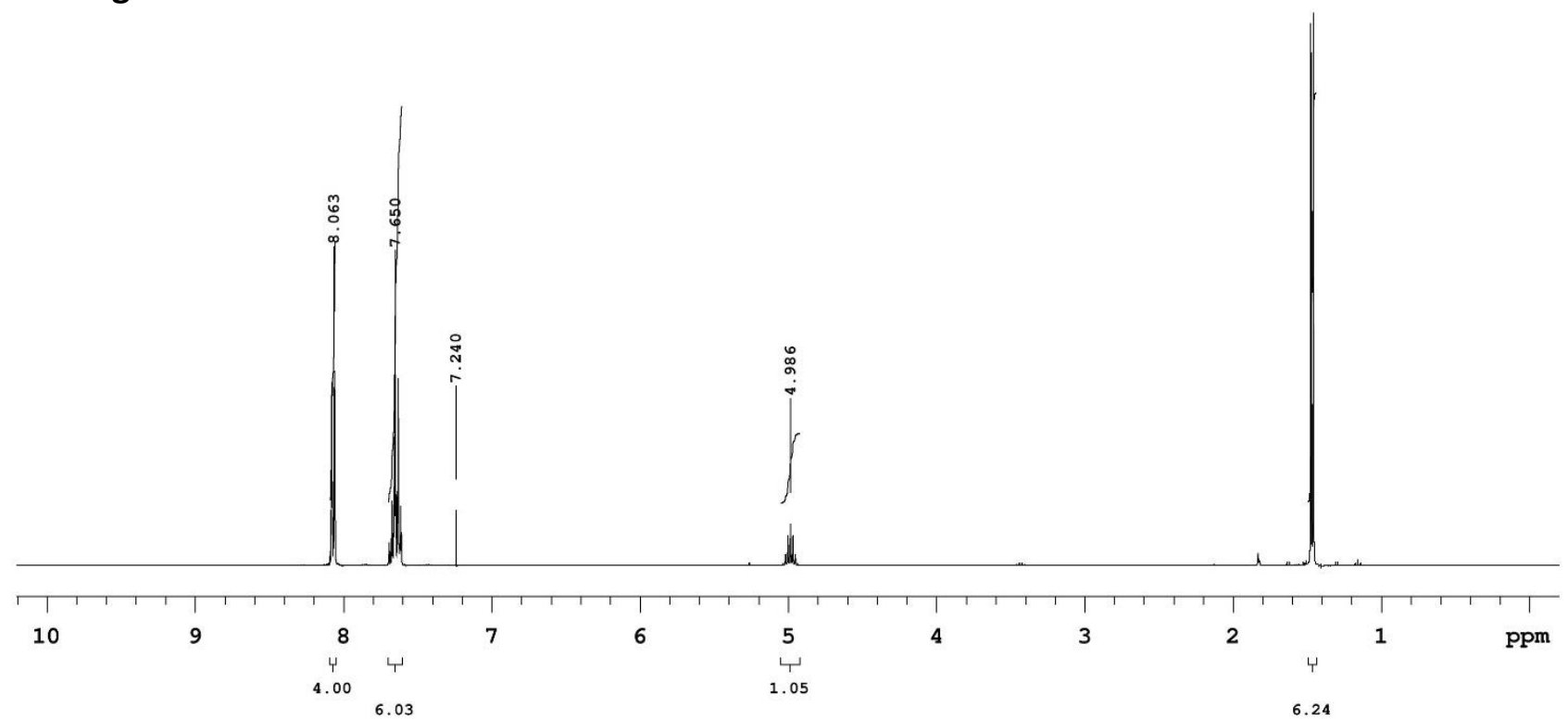

Sample Name: DA-240

Solvent: $\operatorname{cdcl} 3$

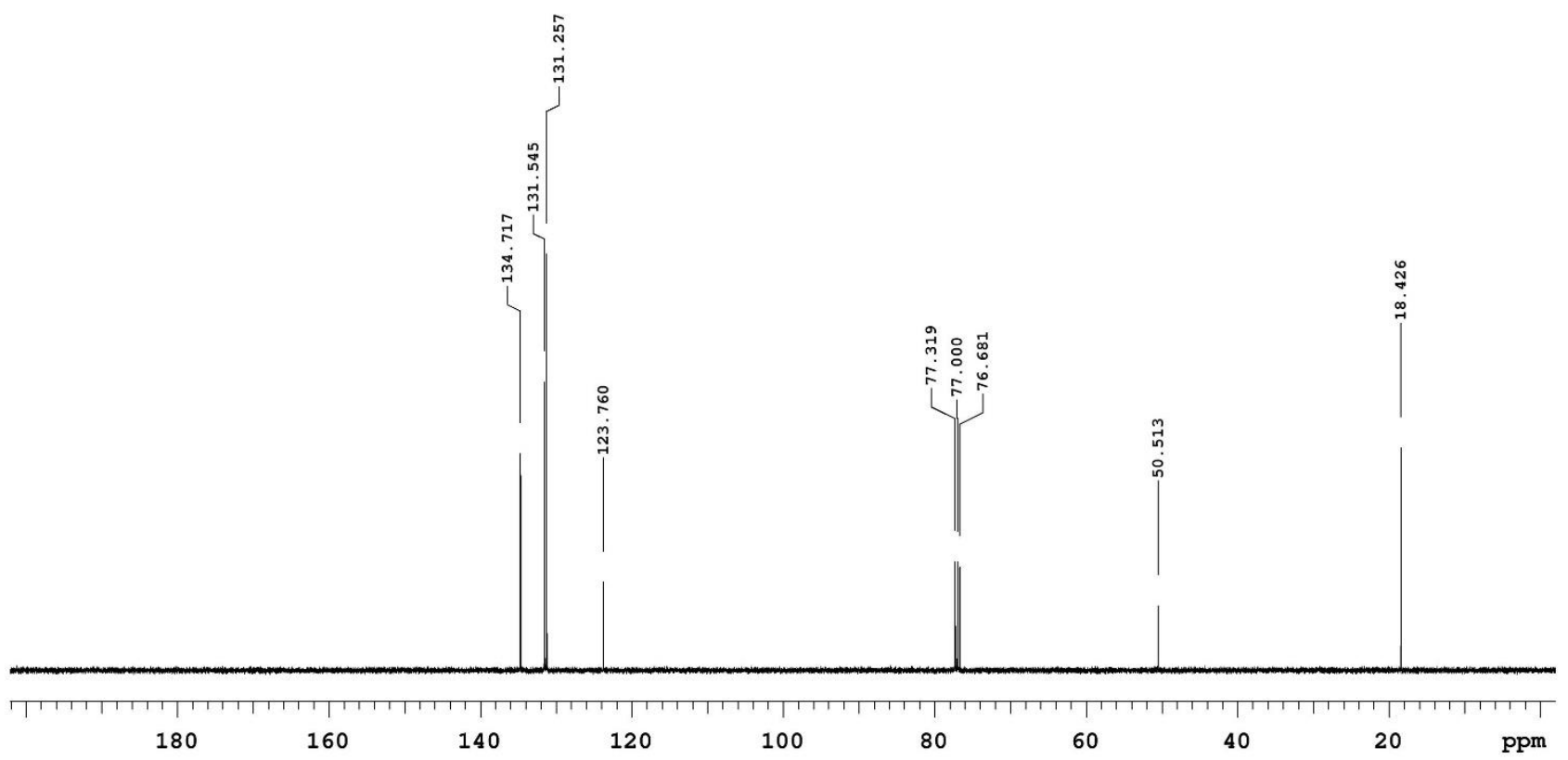


<smiles>O=[N+]([O-])c1cccc2c(Br)cccc12</smiles>

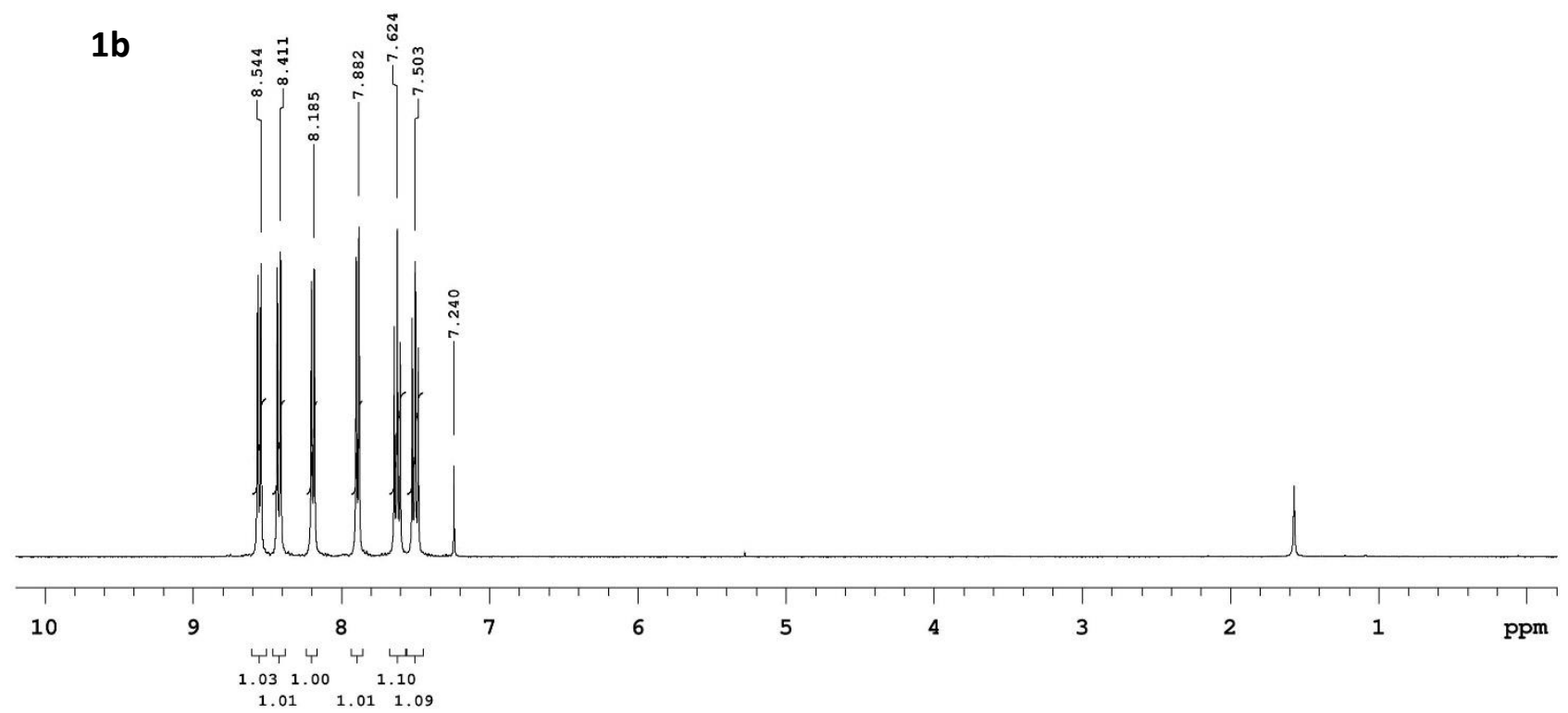

Sample Name: BP-034_kryst2_DCM2

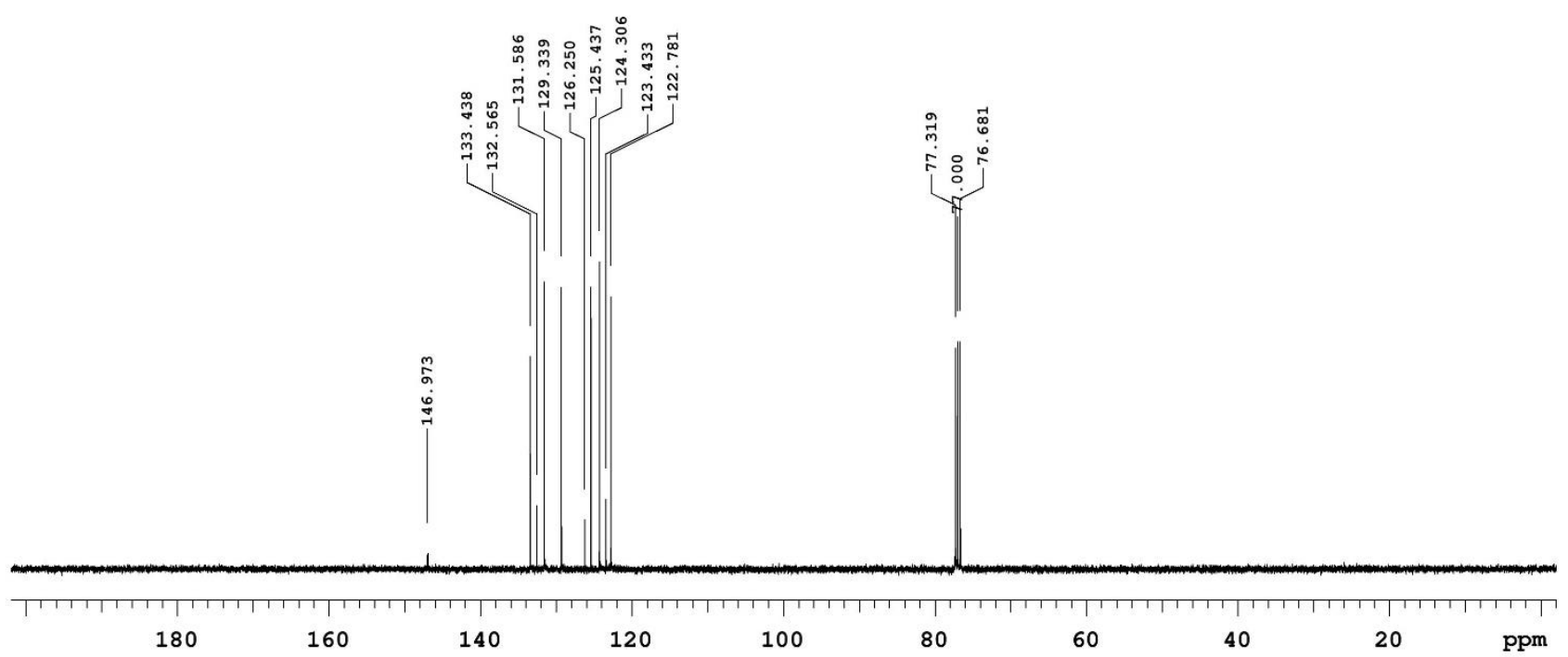


Sample Name: MB-505

Solvent: cdc13<smiles>COc1cccc2ccccc12</smiles>
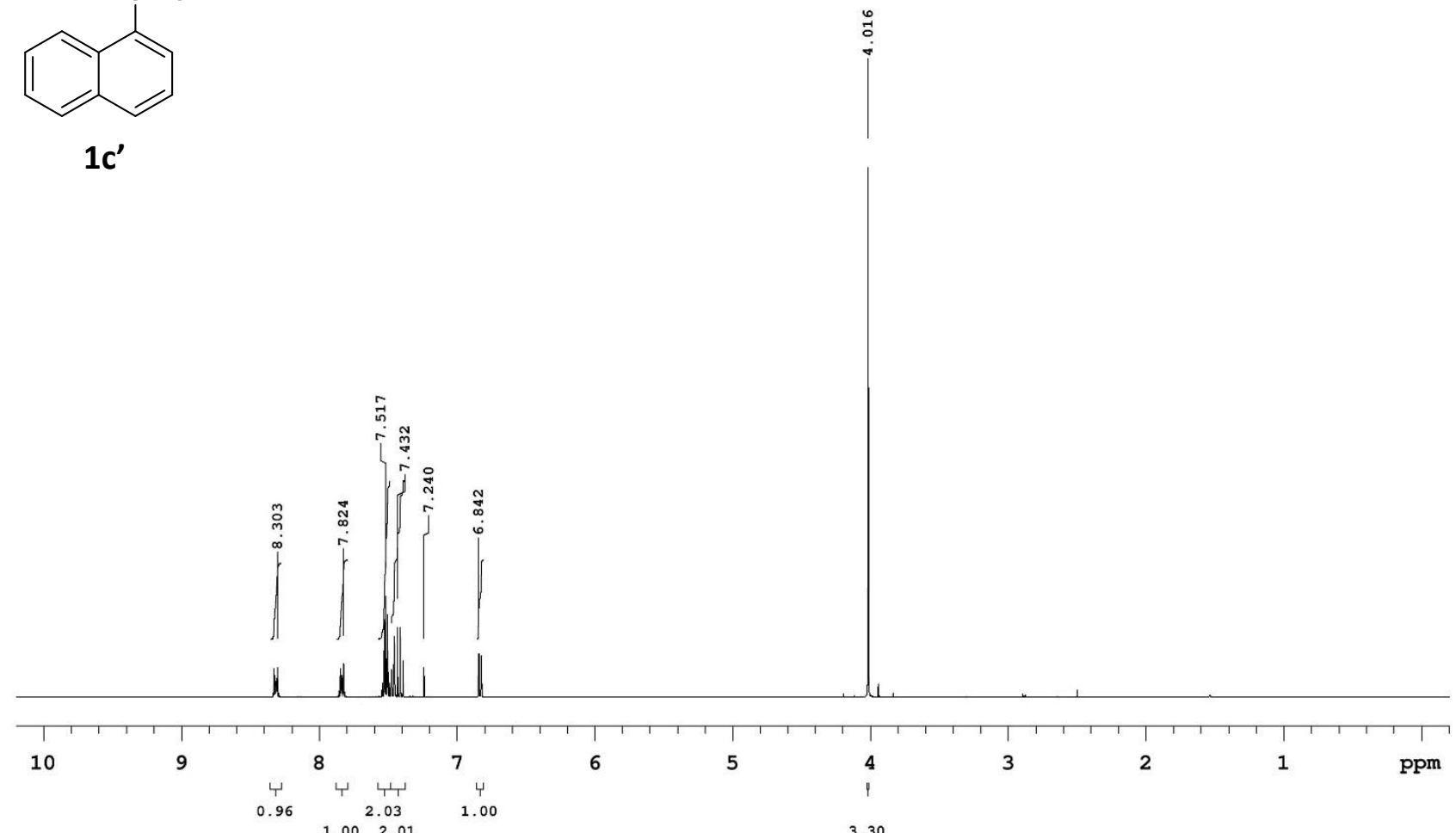

Sample Name: MB-505

Solvent: $\operatorname{cdcl} 3$

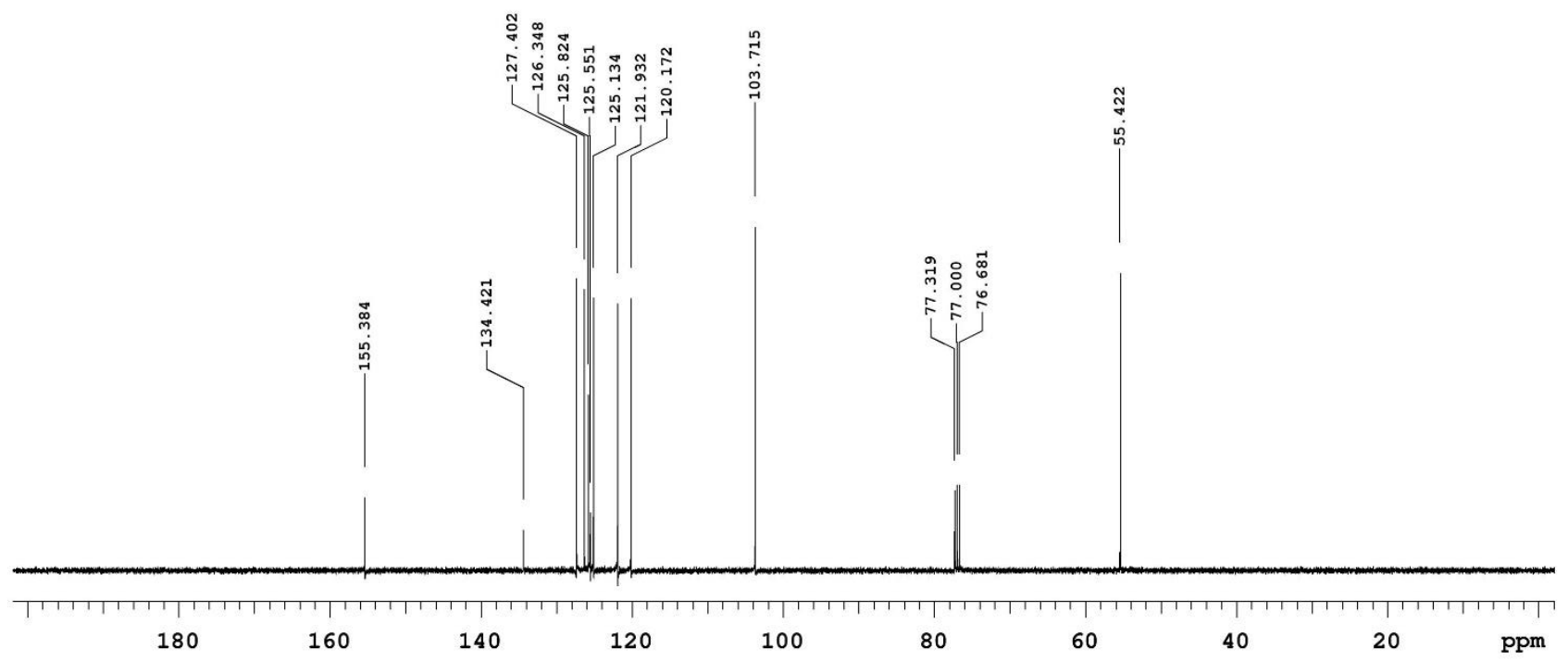


<smiles>COc1ccc([N+](=O)[O-])c2ccccc12</smiles>

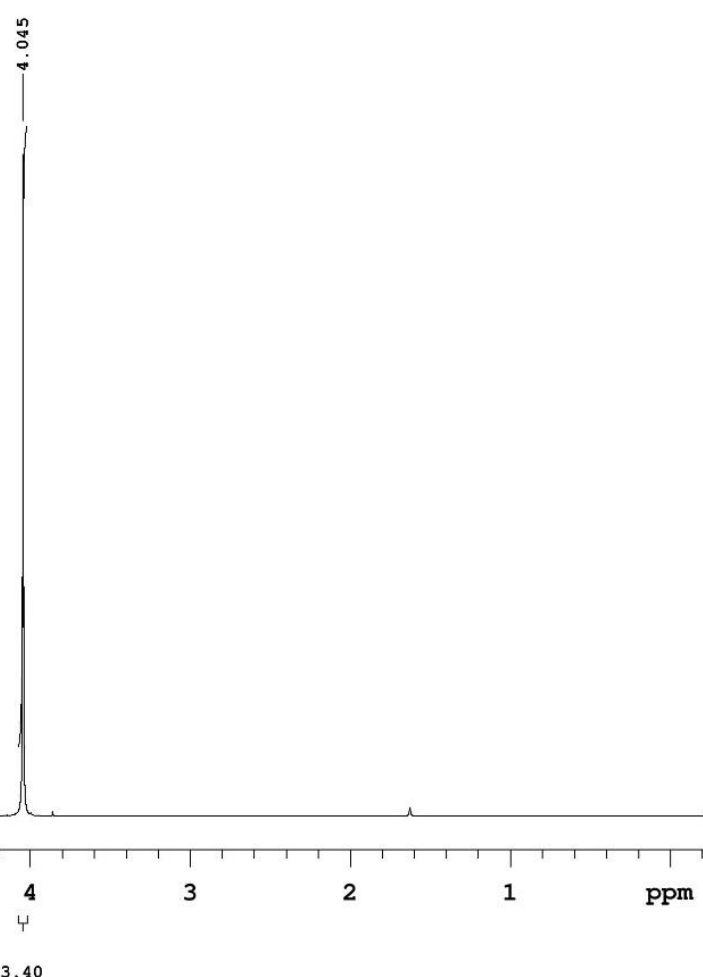

Sample Name: TB-187XB Solvent: cdc13

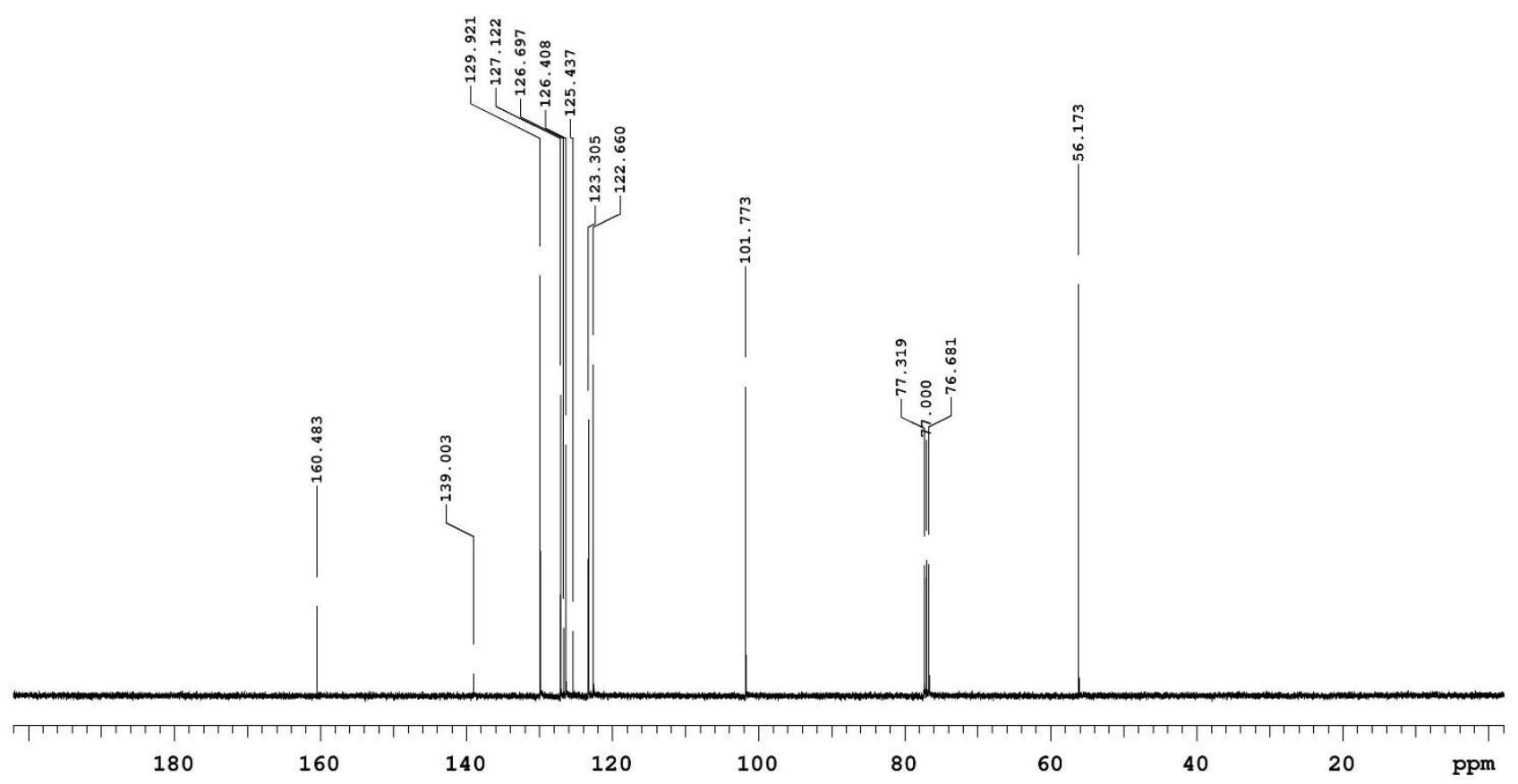


Sample Name: DA-286b

Solvent: cdc13
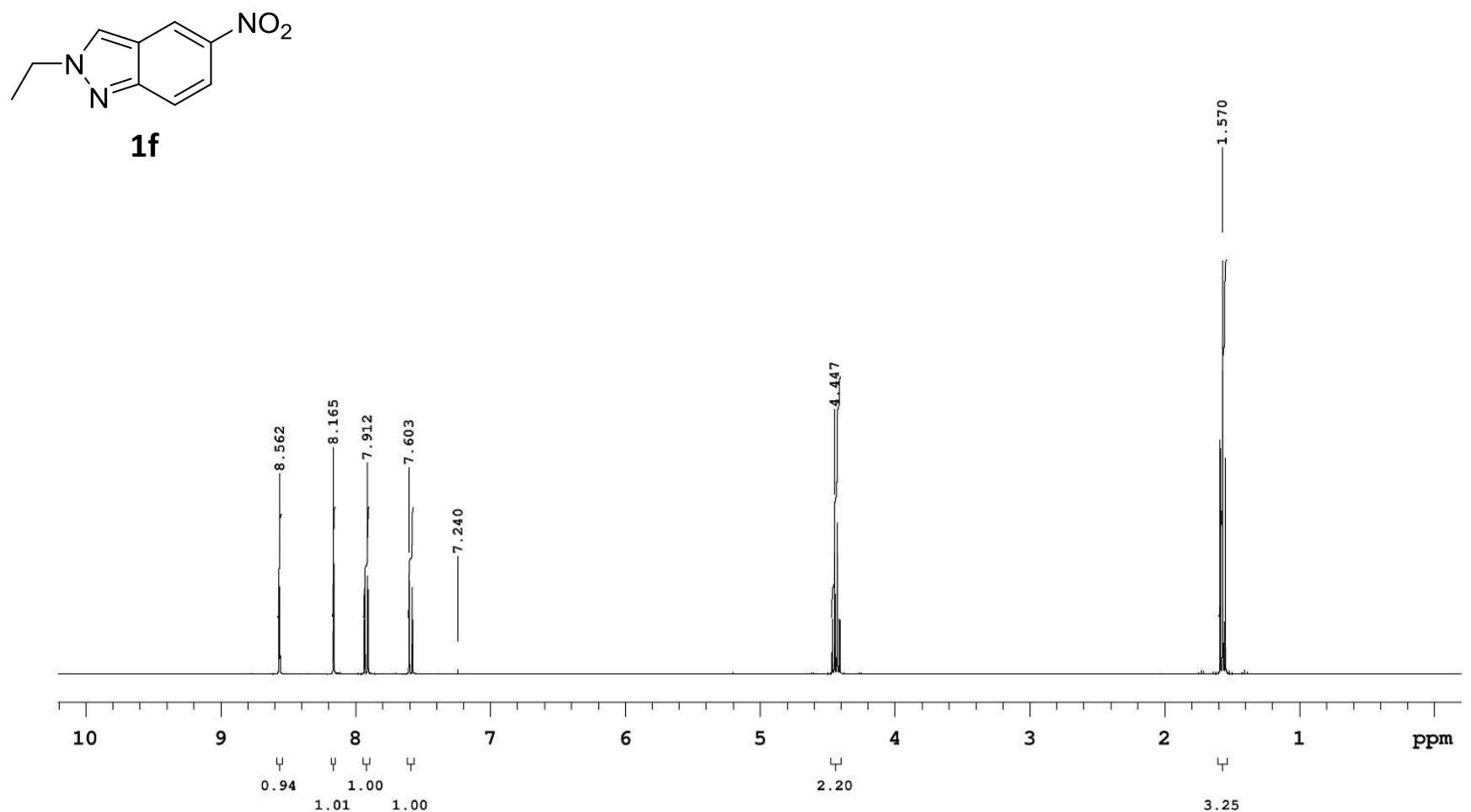

Sample Name: DA-286b

Solvent: cdc13

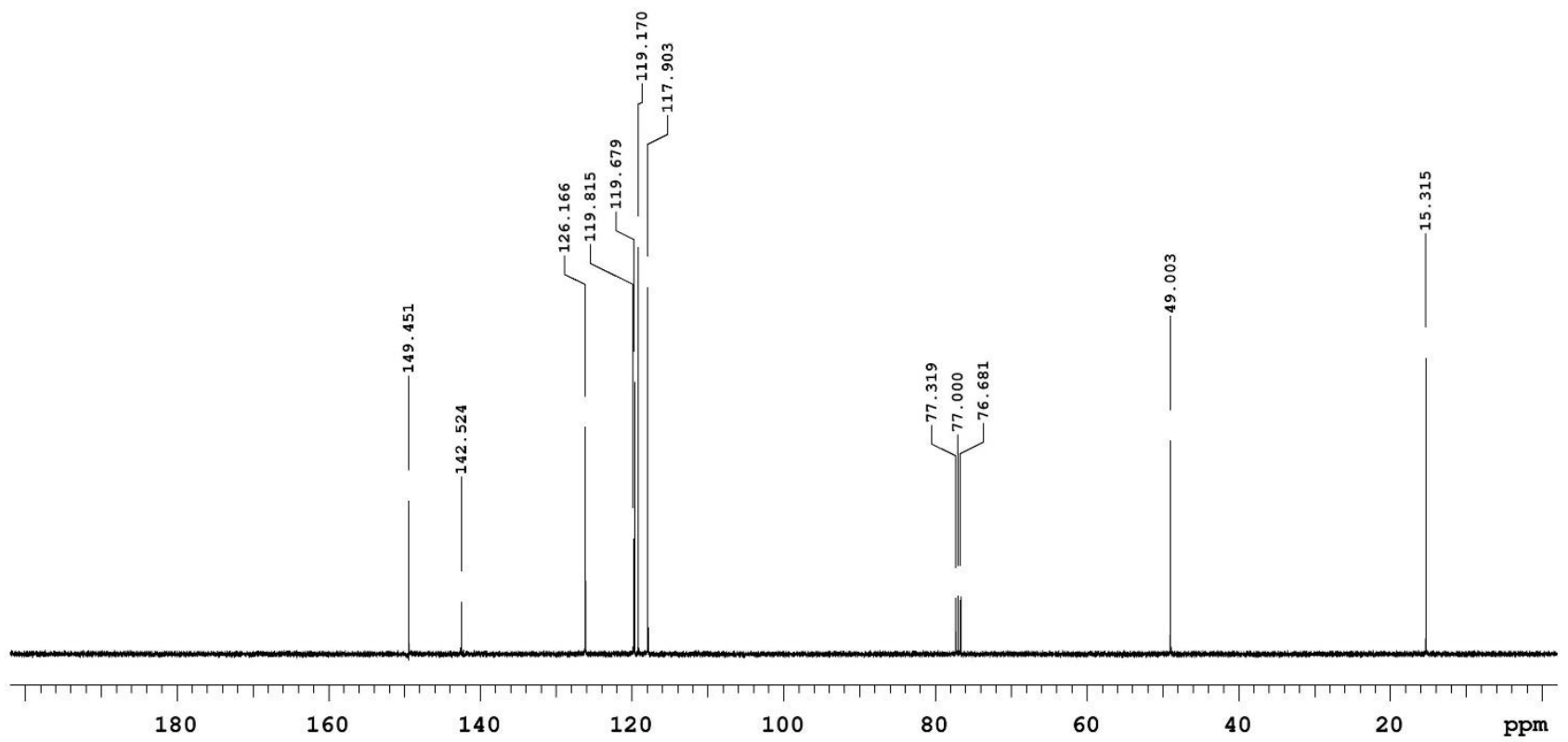


Sample Name: DA-286a

Solvent: cdc13

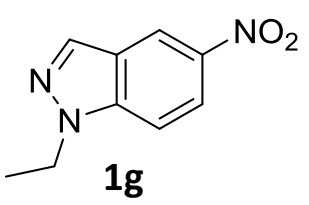

Sample N

Solvent: cdc13

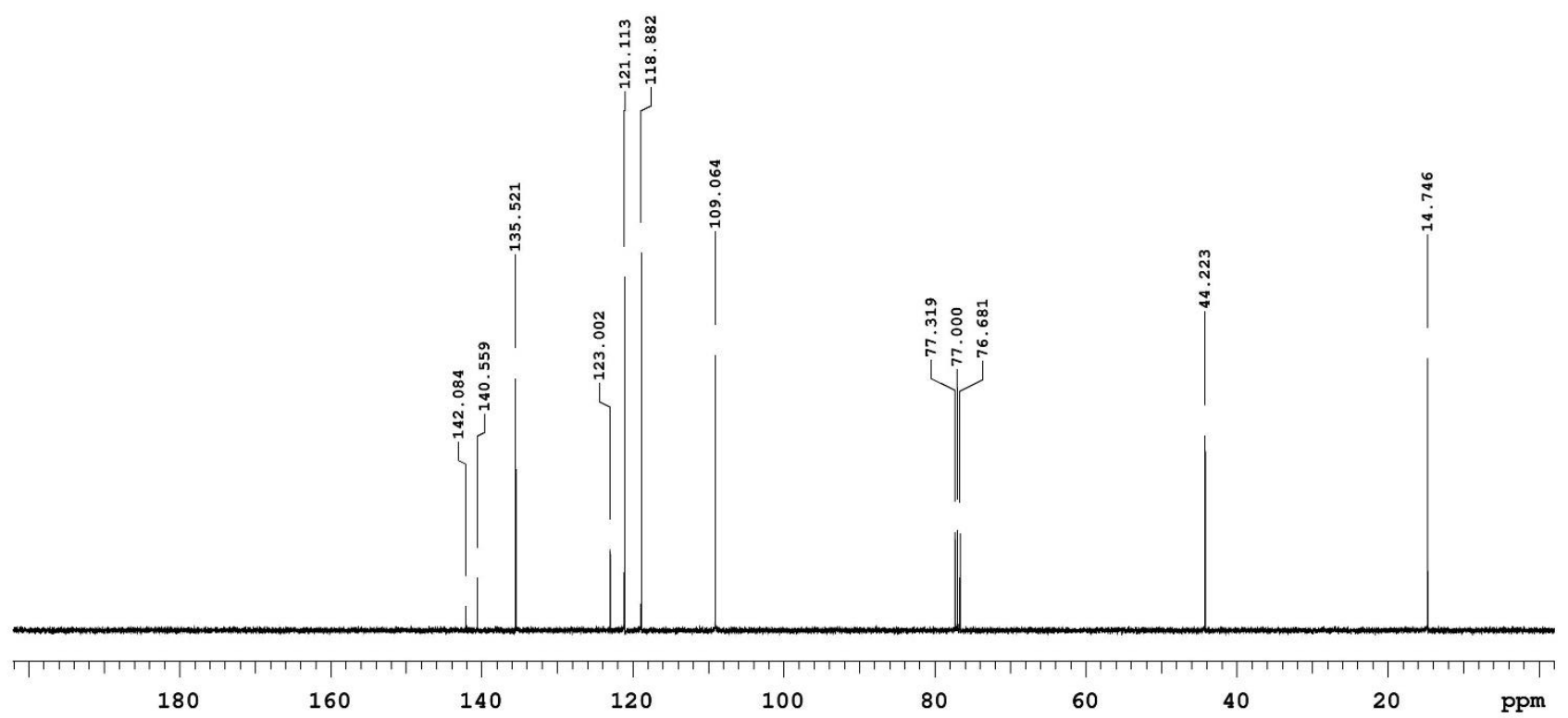


Sample Name: TB-142A

Solvent: cdc13

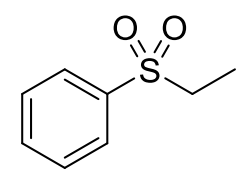

5

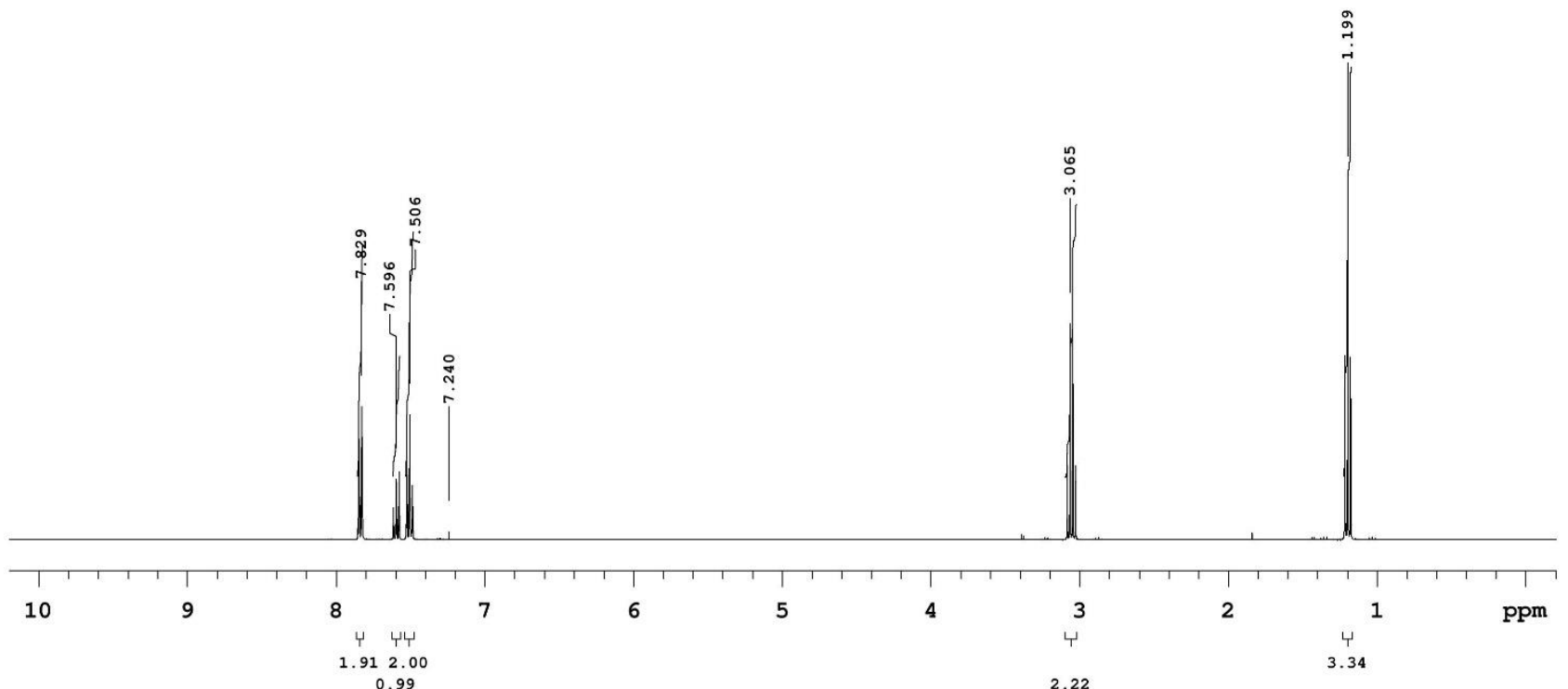

Sample Name: TB-142A

Solvent: $\operatorname{cdcl} 3$

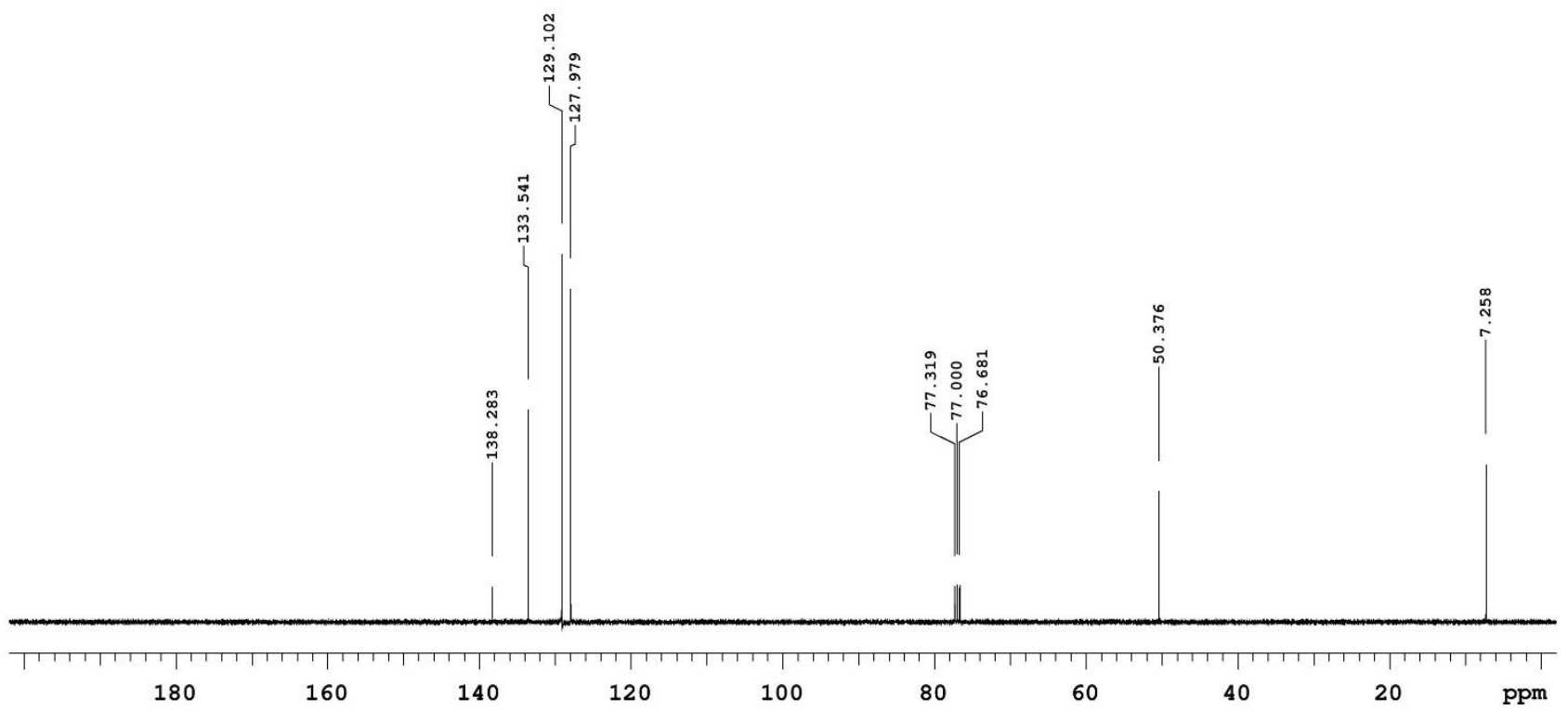




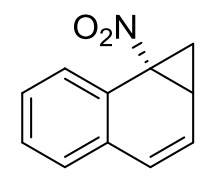

$3 a$

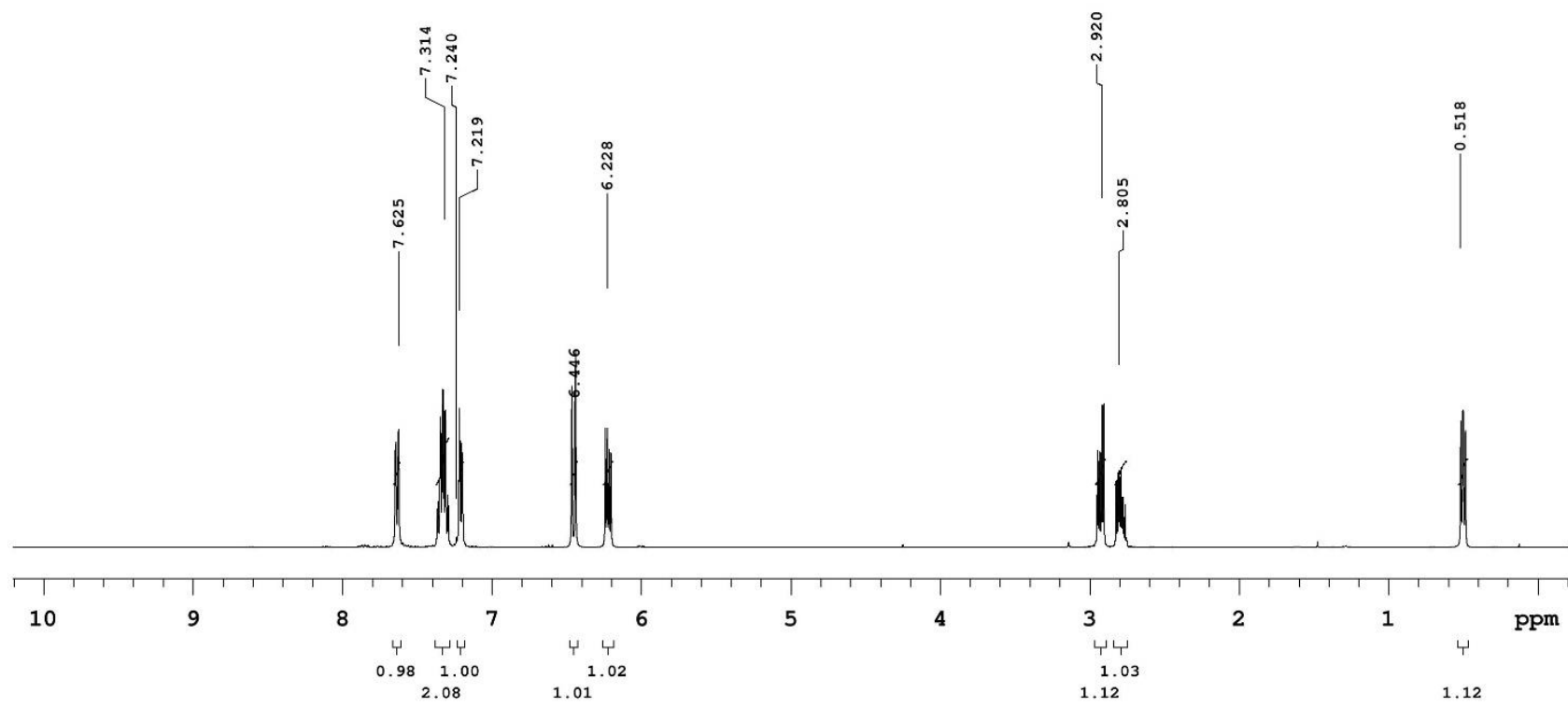

Sample Name: DA-231d

Solvent: $\operatorname{cdcl} 3$

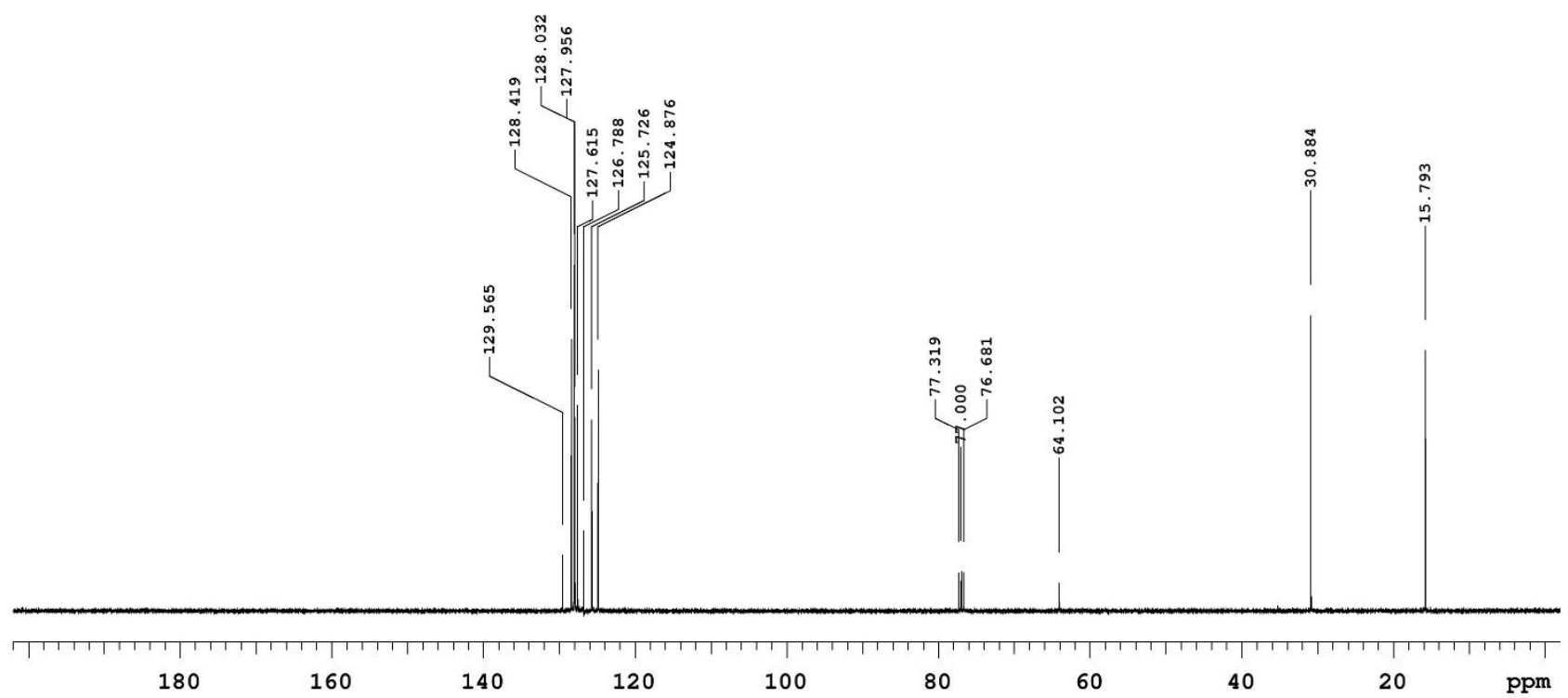


Sample Name: DA-165c3

Solvent: cdc13

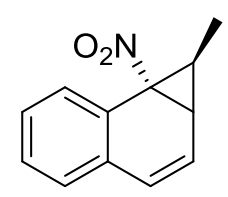

endo-3b

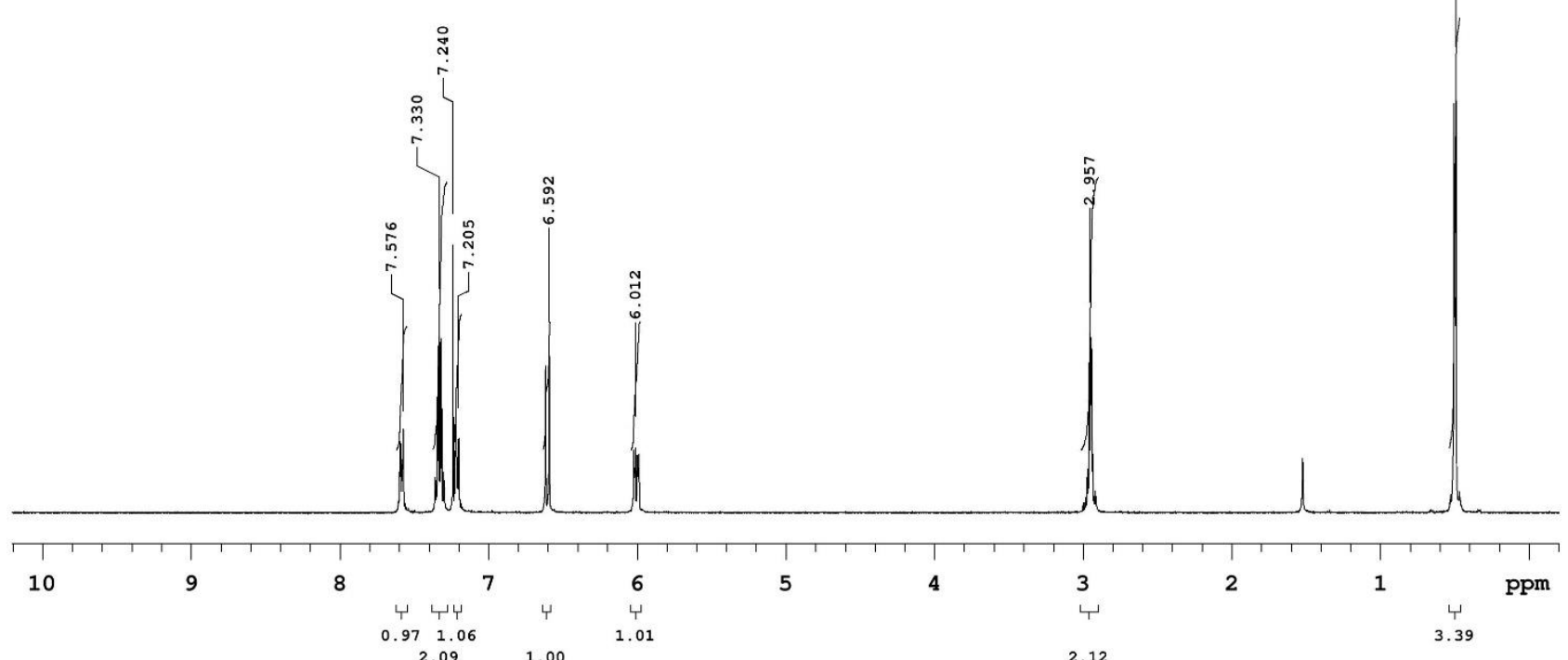

Sample Name: DA-165c

Solvent: cdc13

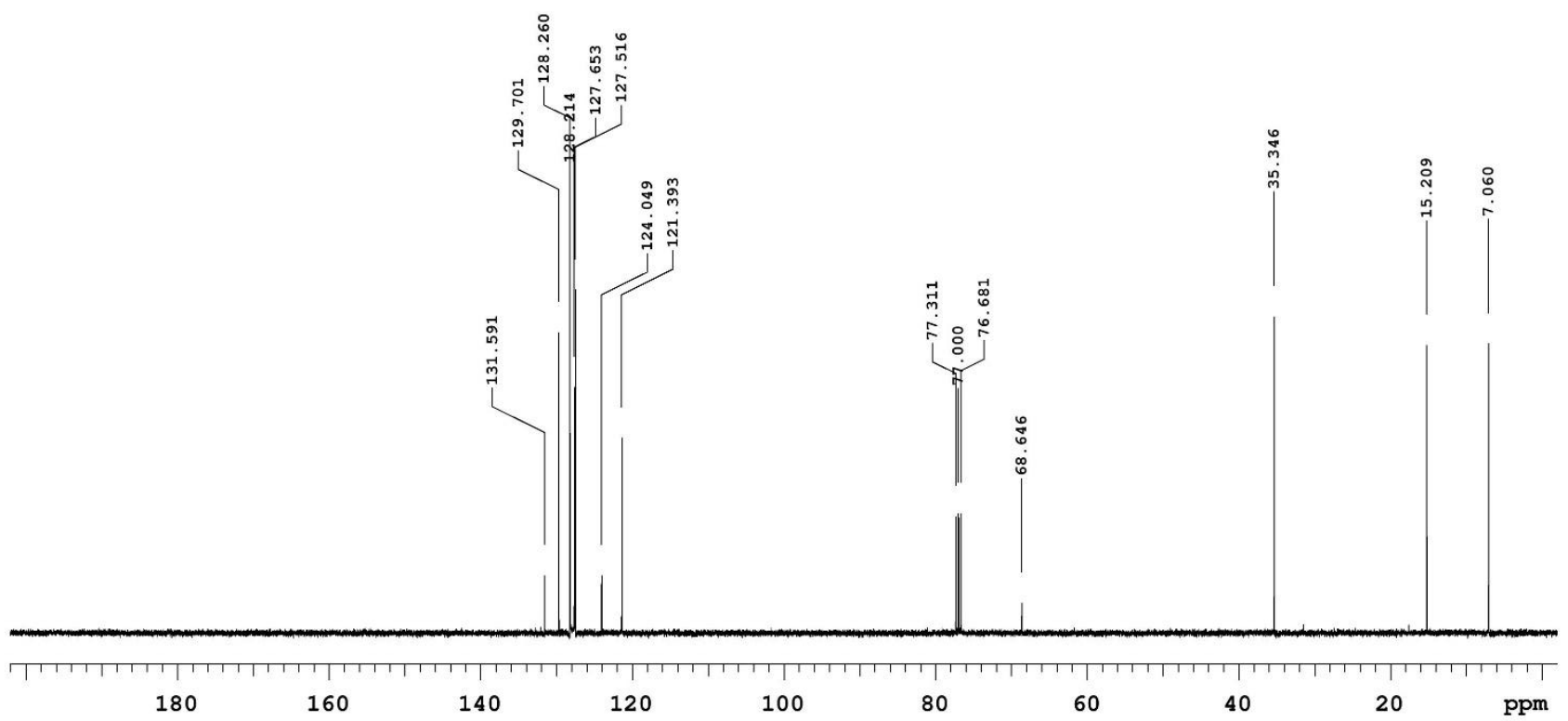




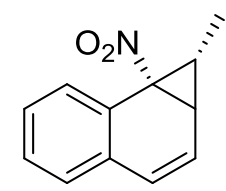

exo-3b

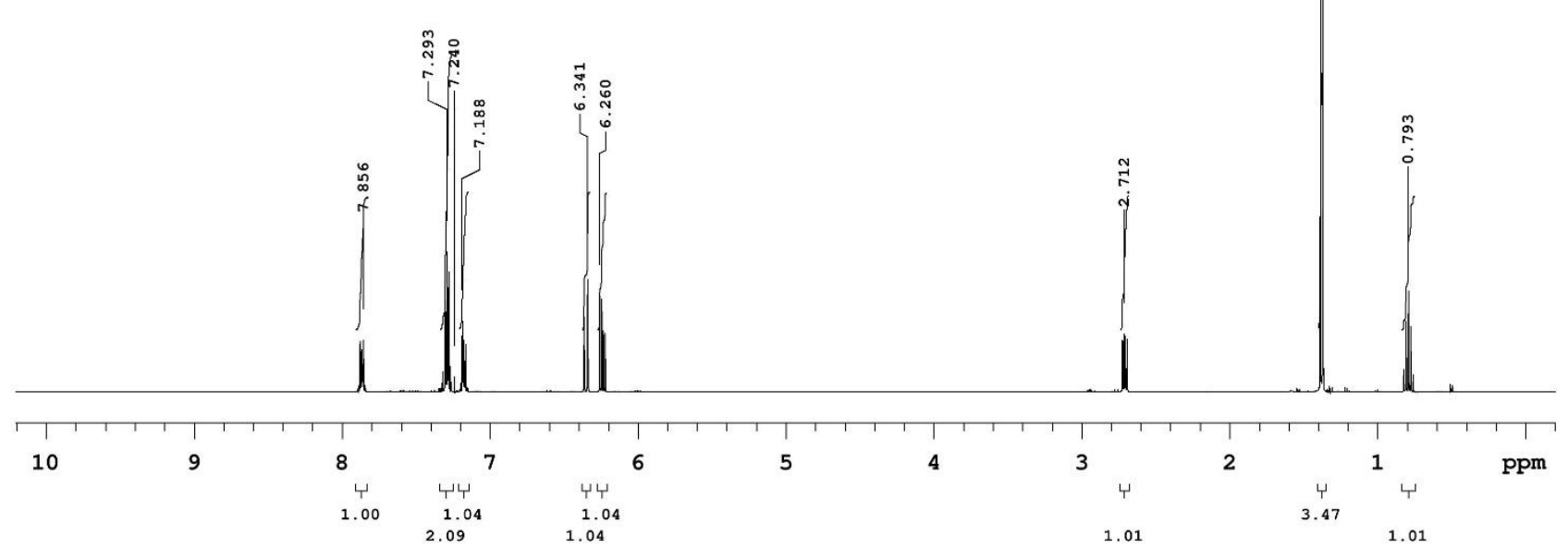

Sample Name: DA-202C

Solvent: cdc13

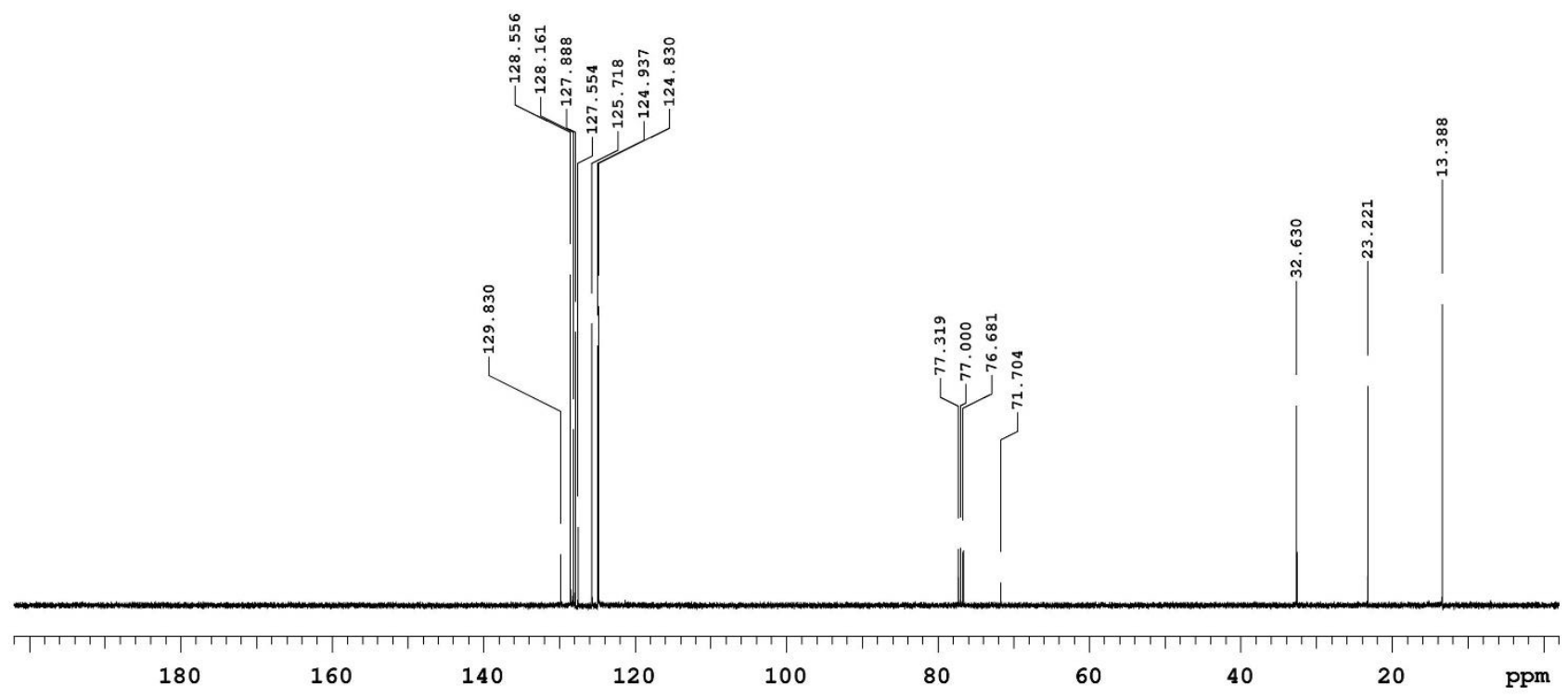


Sample Name: DA-263e

Solvent: cdc13

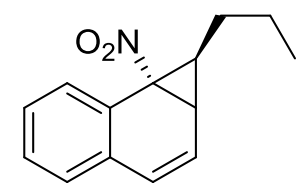

endo-3c

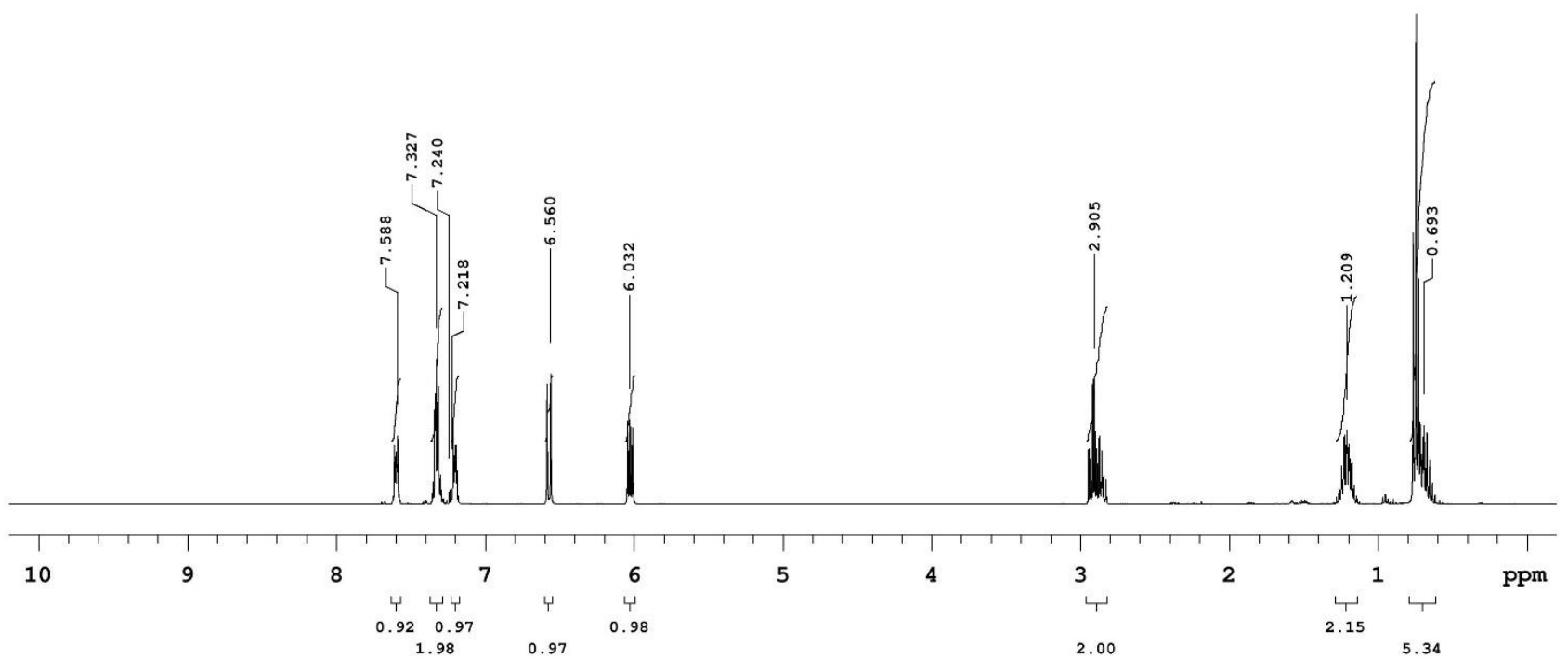

Sample Name: DA-263e

Solvent: cdc13

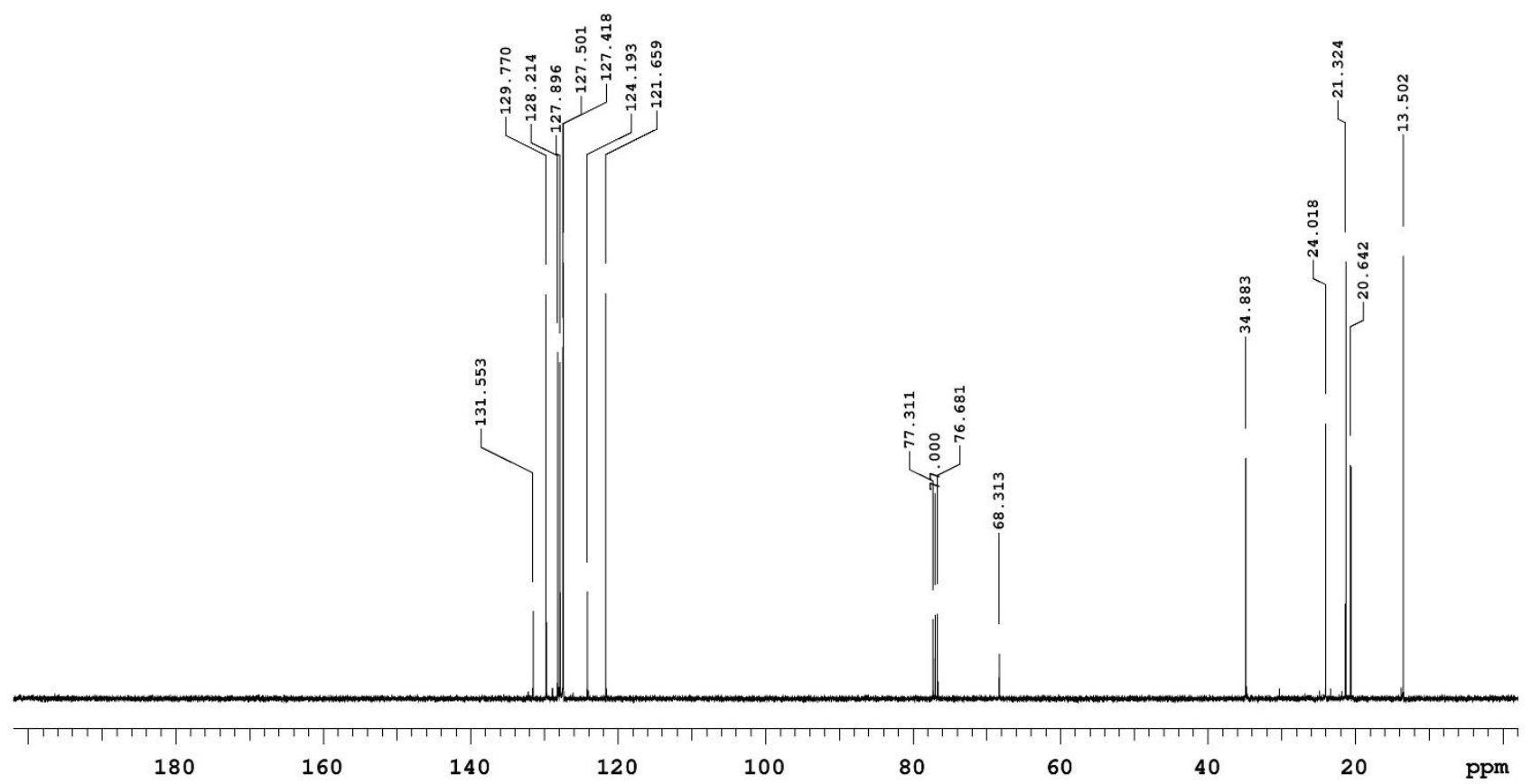



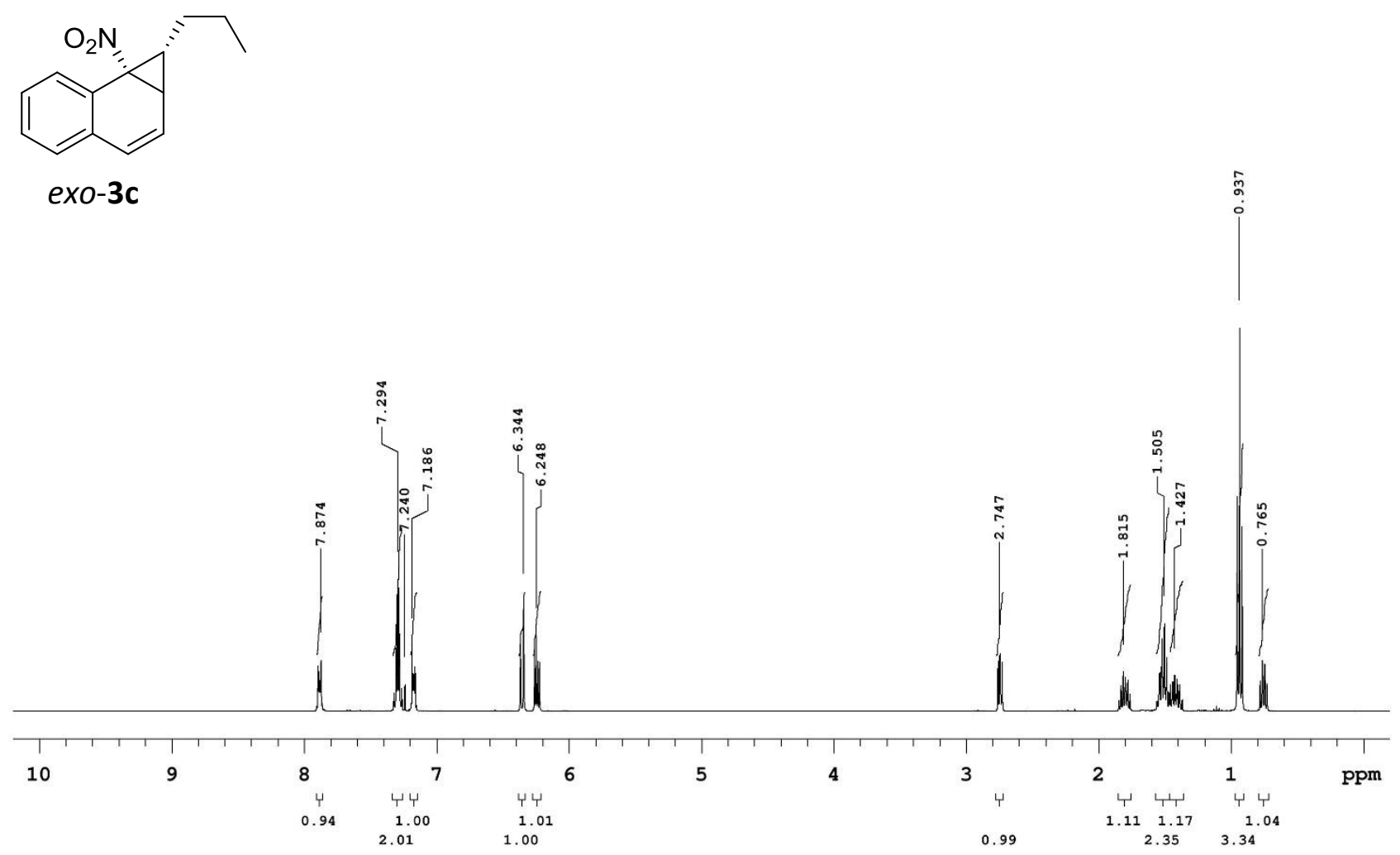

Sample Name: DA-263b

Solvent: cdc13

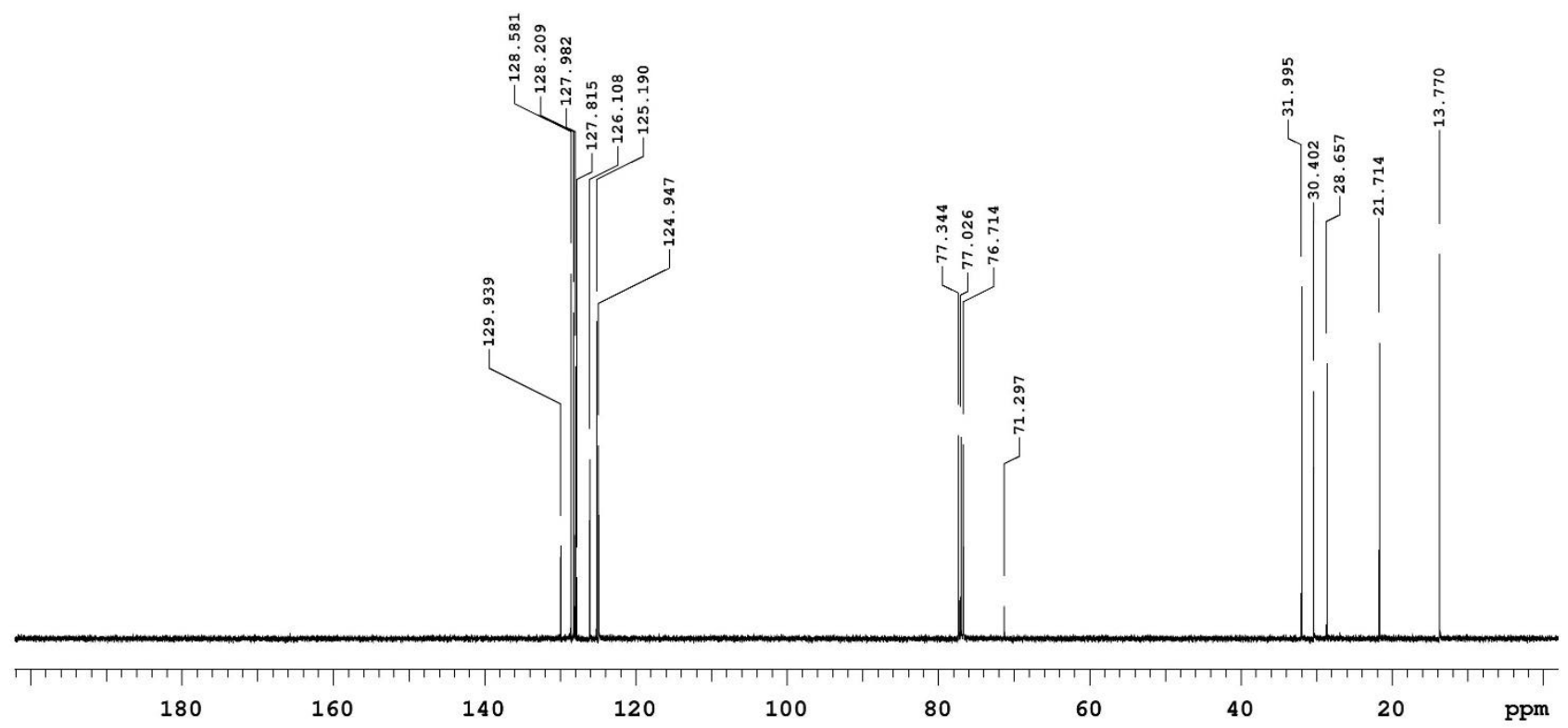




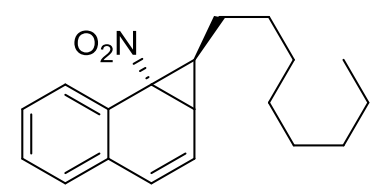

endo-3d

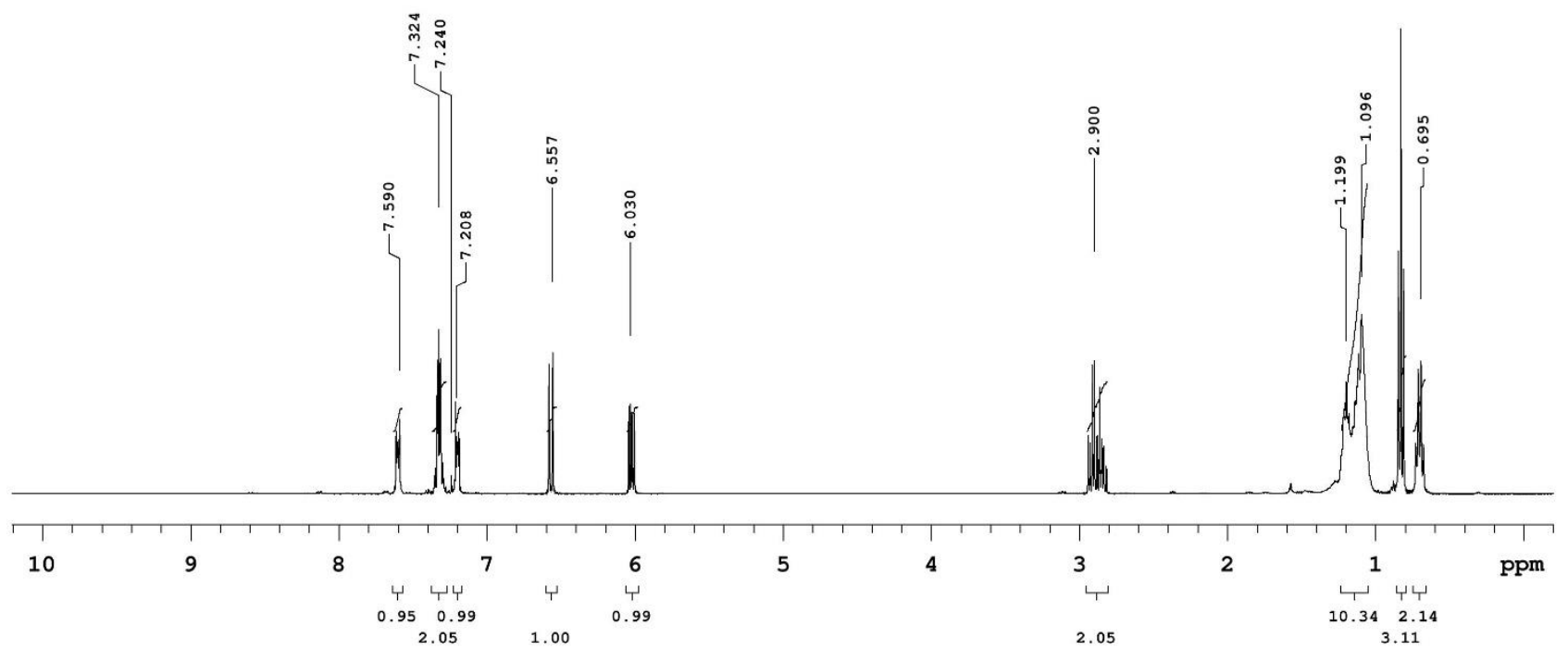

Sample Name: DA-167c

Solvent: cdc13

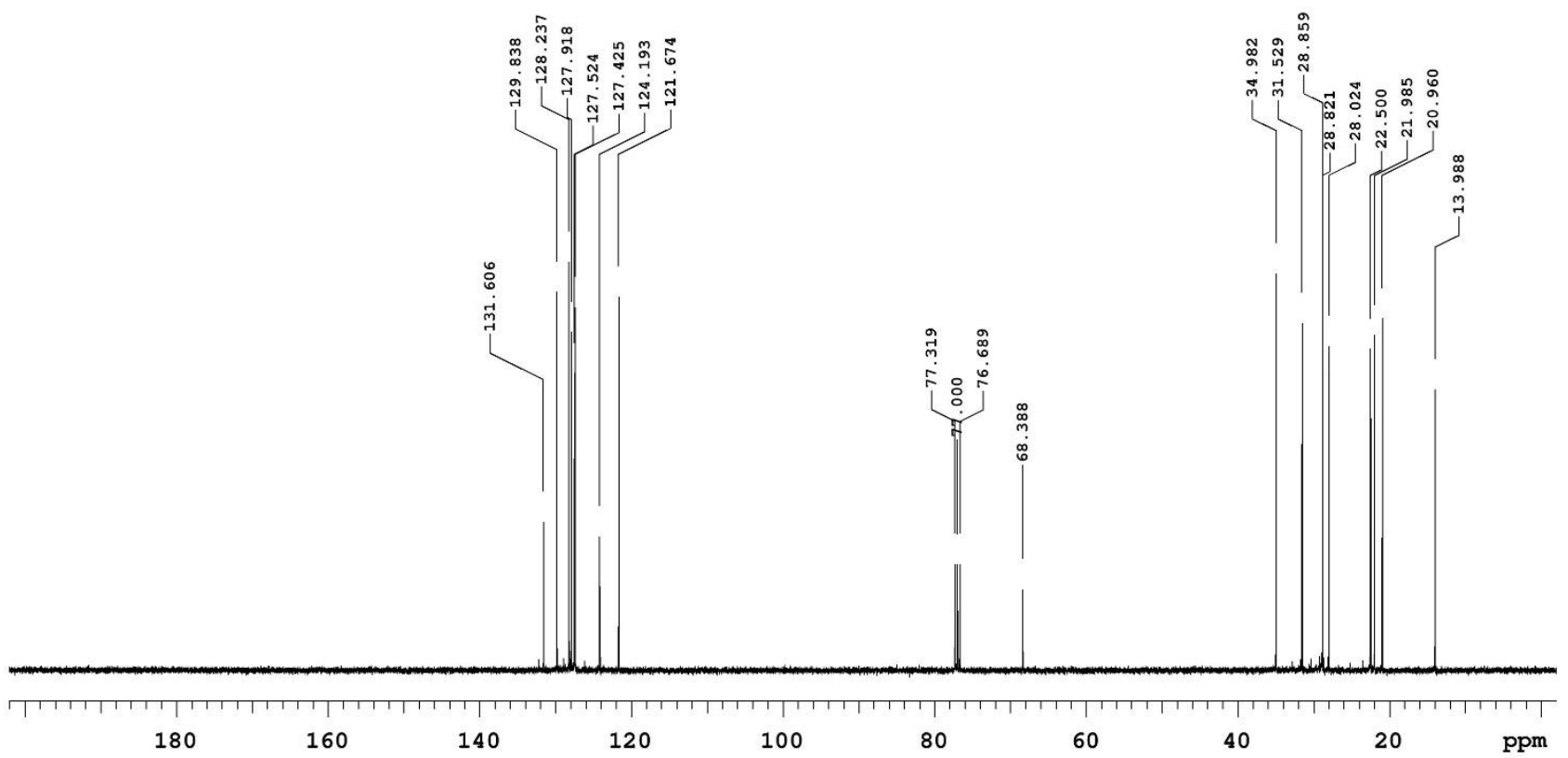



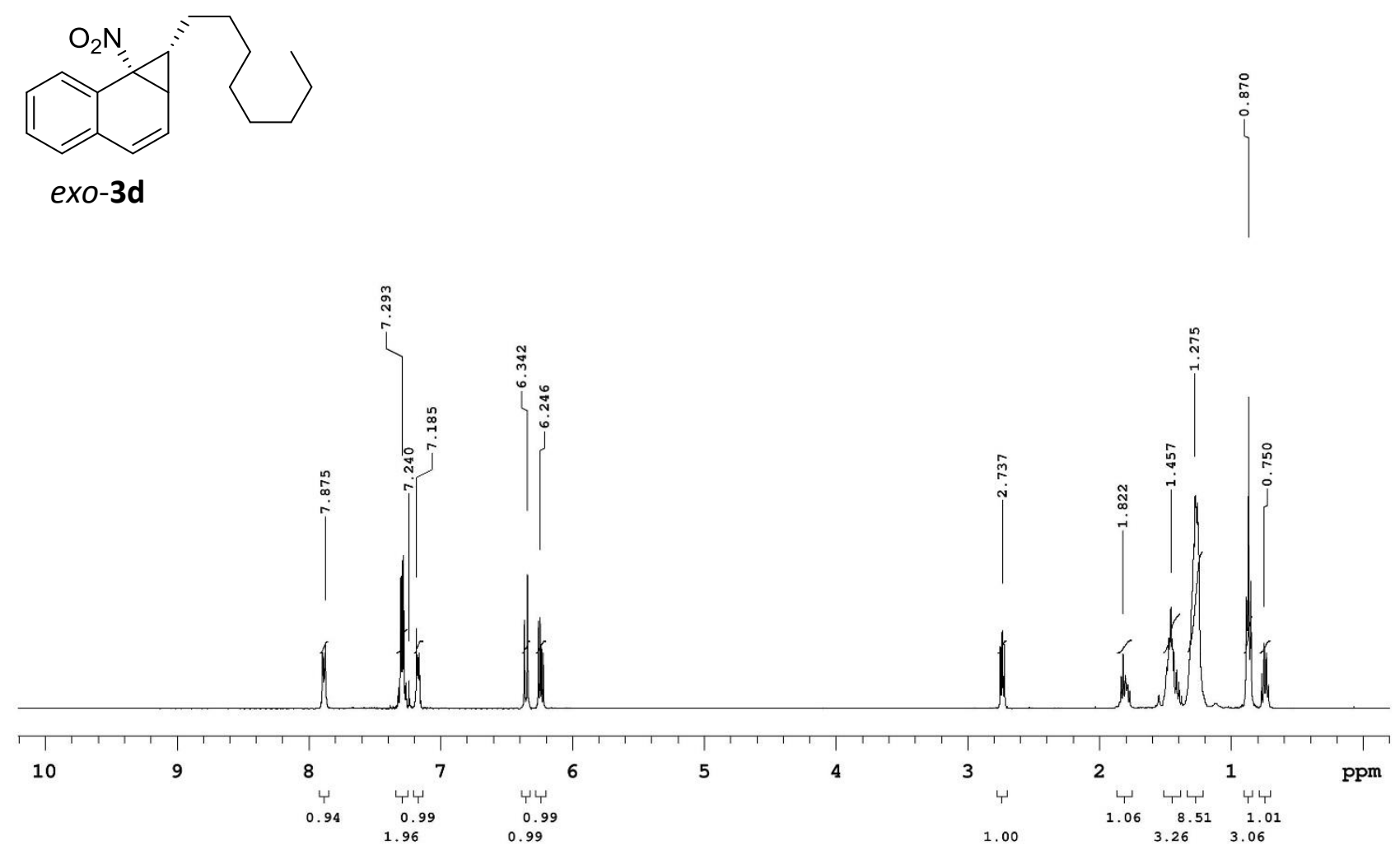

Sample Name: DA-167b

Solvent: cdc13

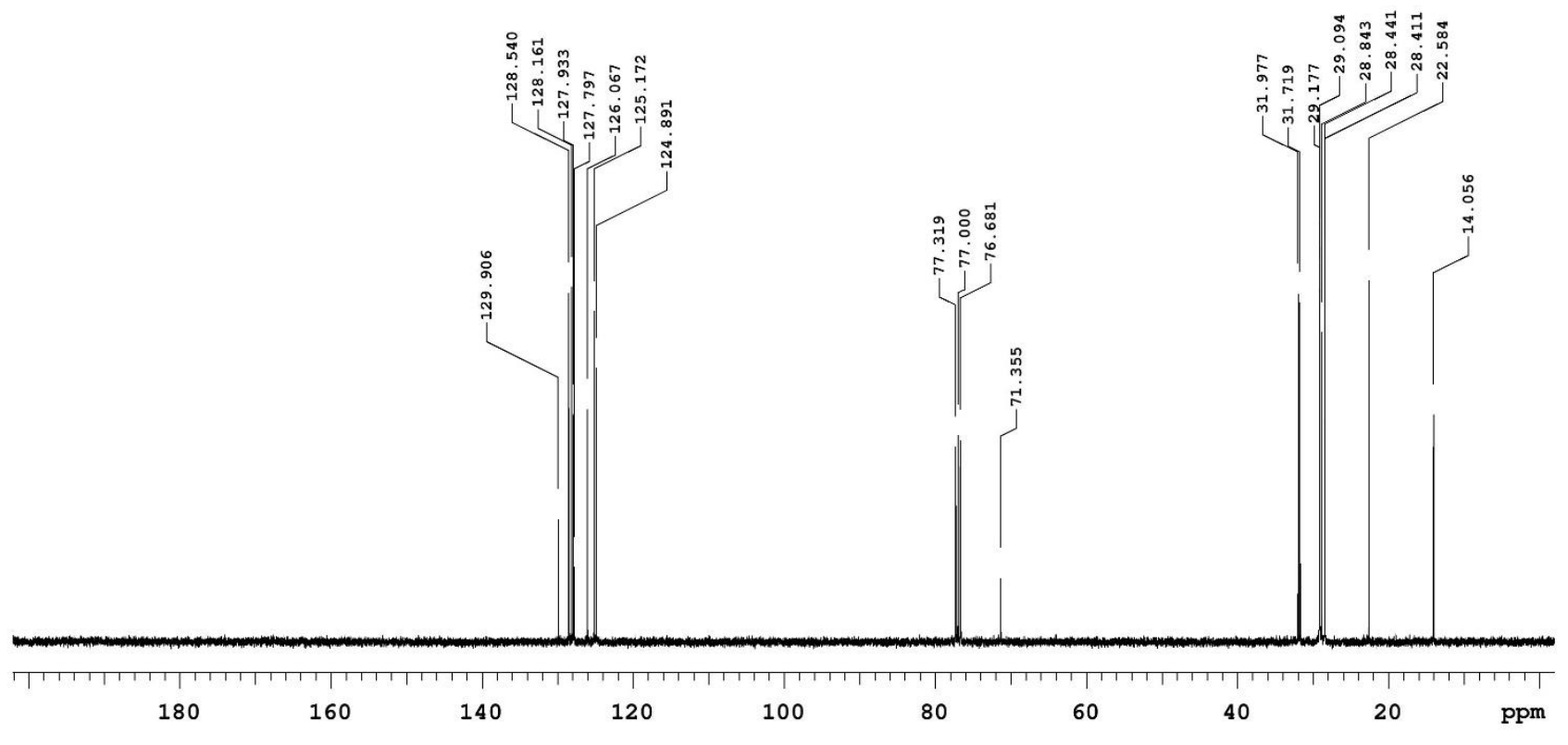




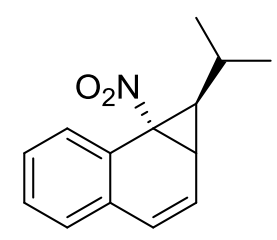

endo-3e
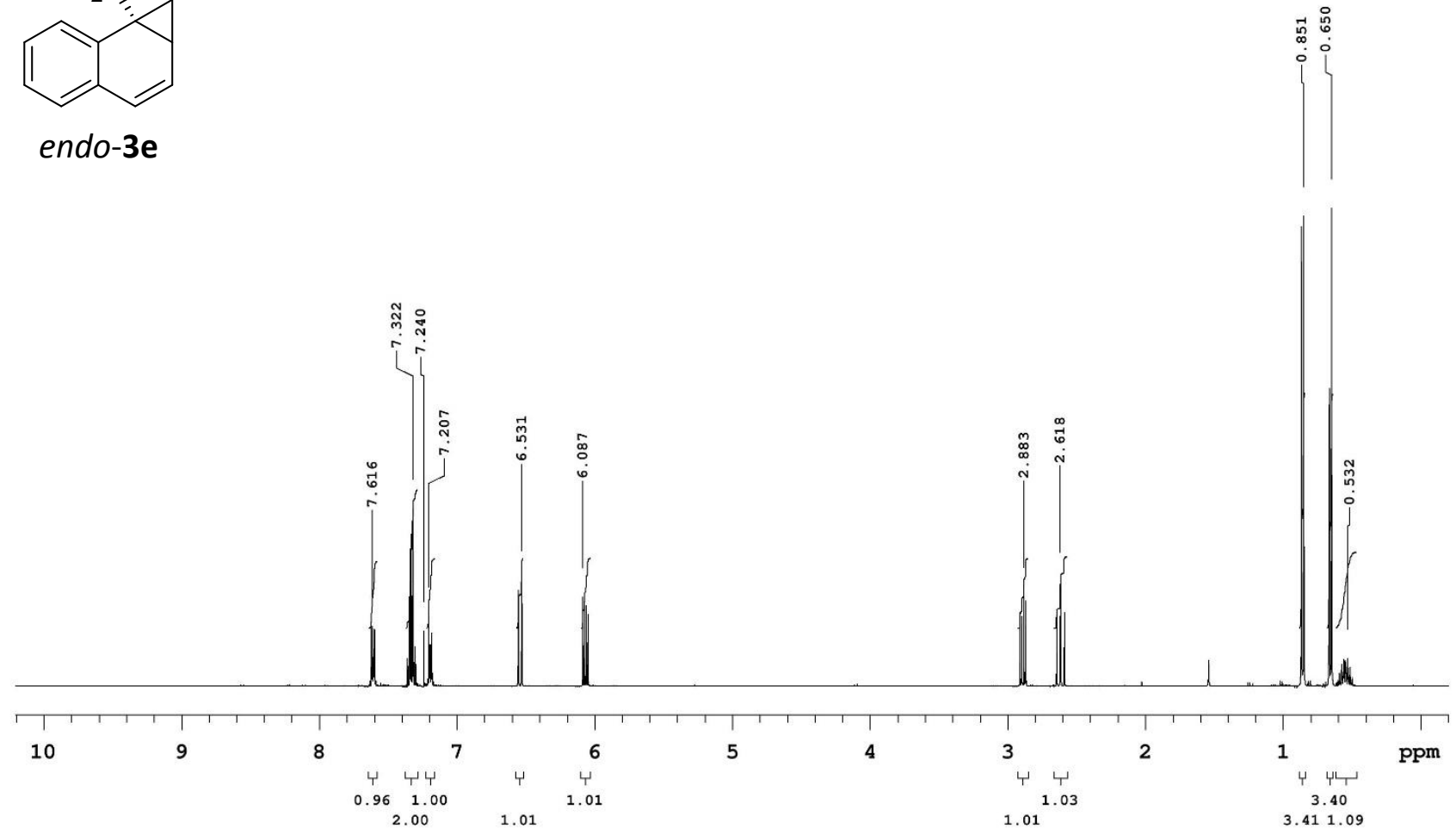

Sample Name: DA-180c2 Solvent: cdc13

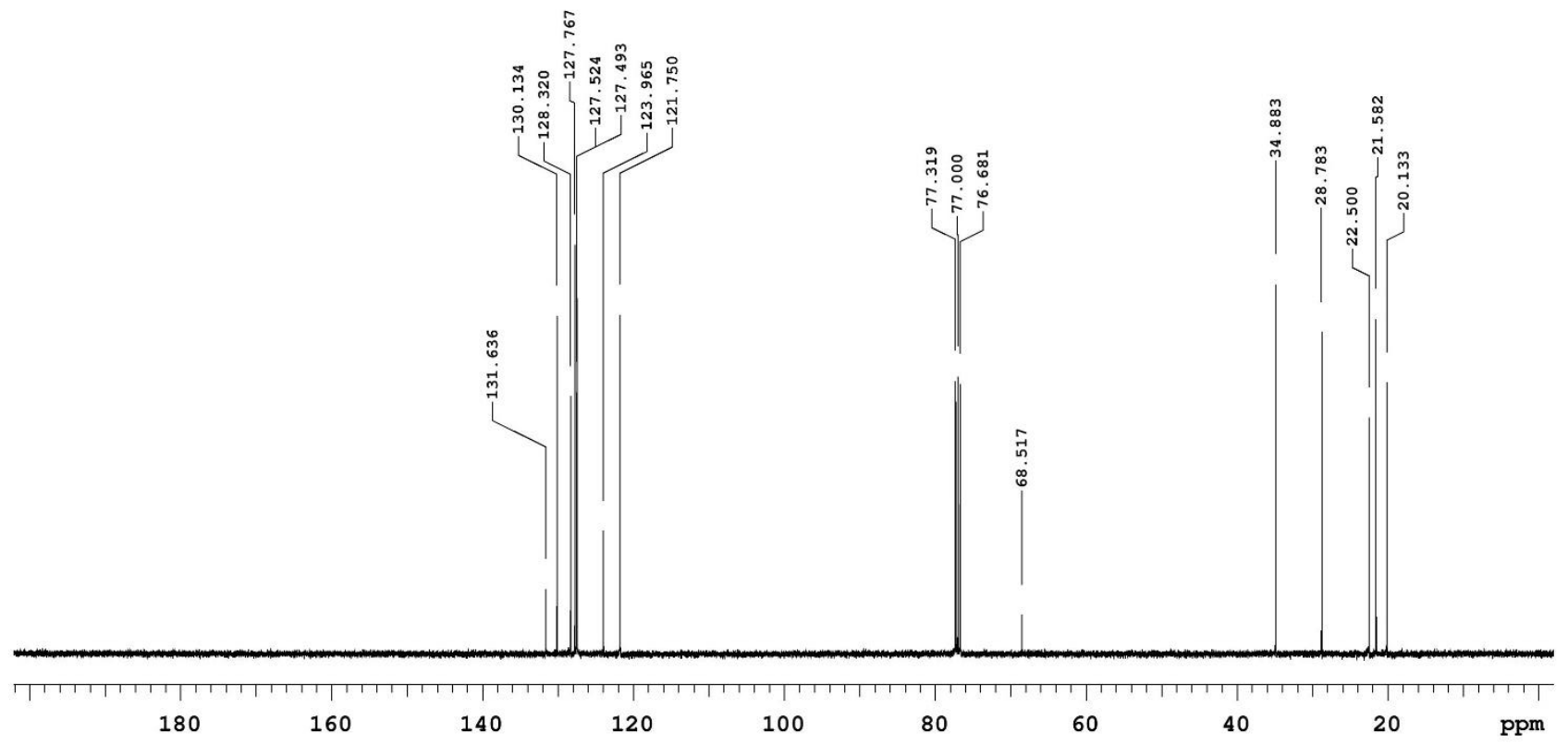


Sample Name: DA-180bb

Solvent: $\operatorname{cdc13}$
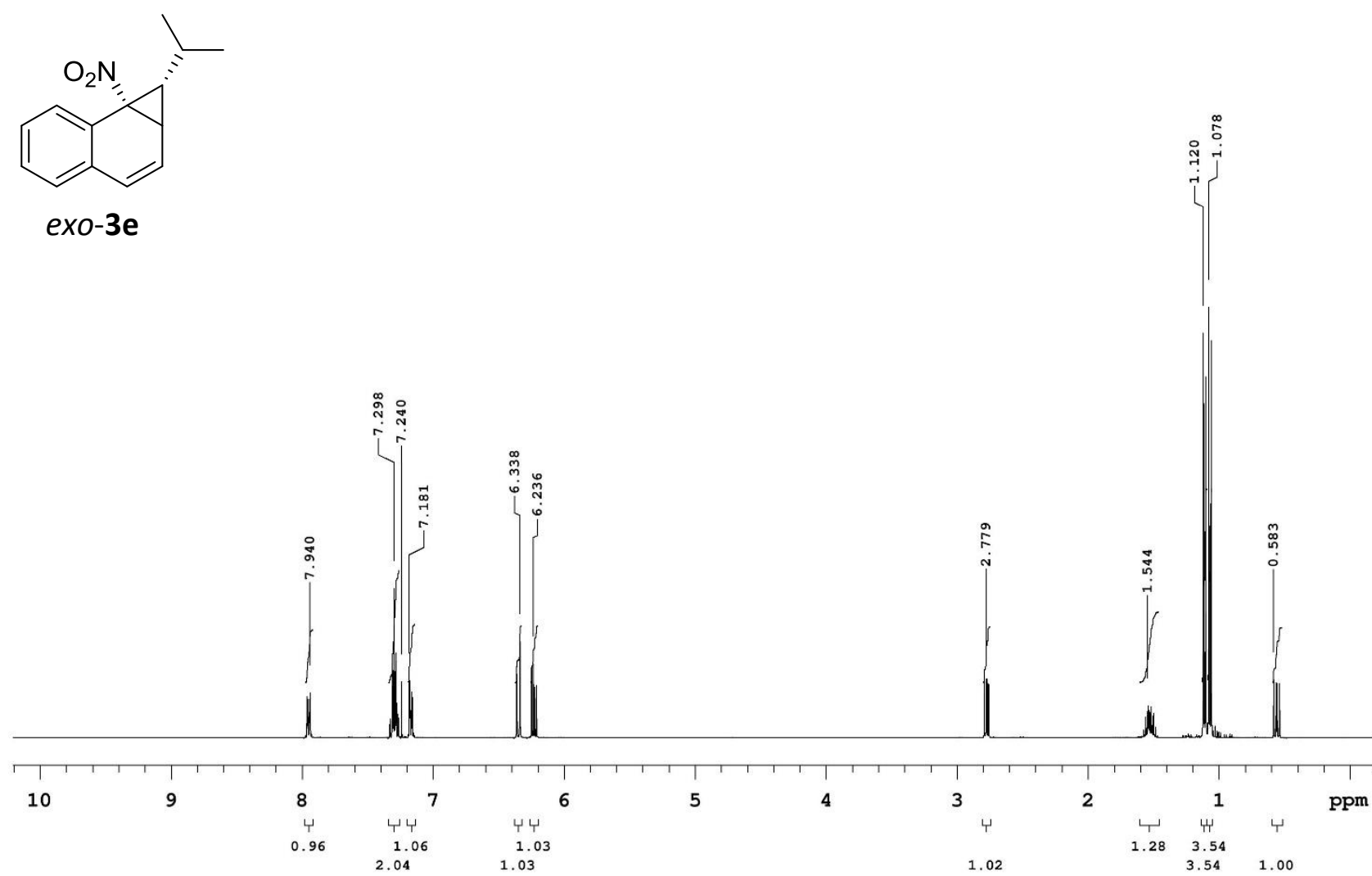

Sample Name: DA-180bb

Solvent: cdc13

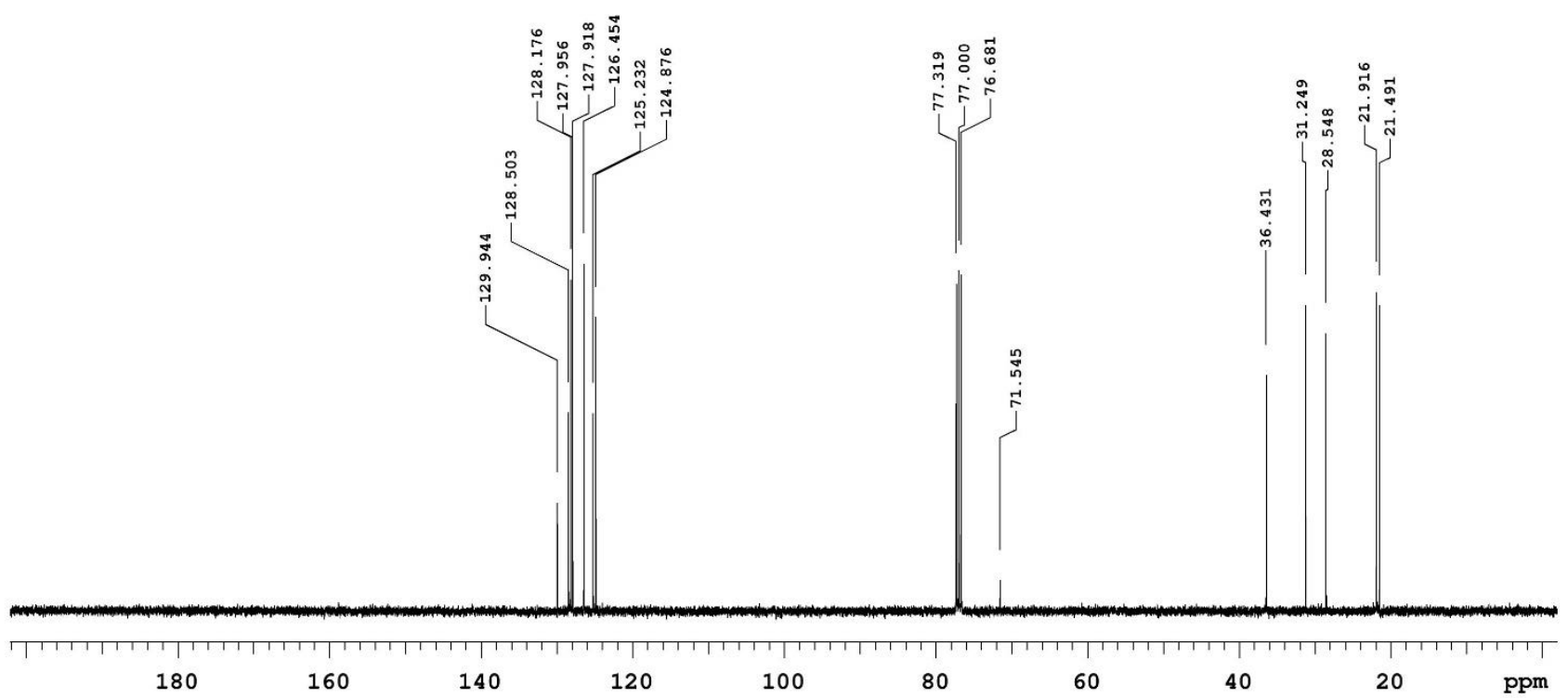




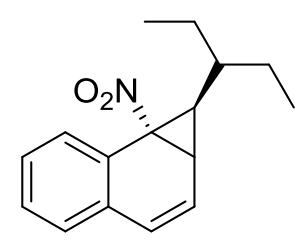

endo-3f
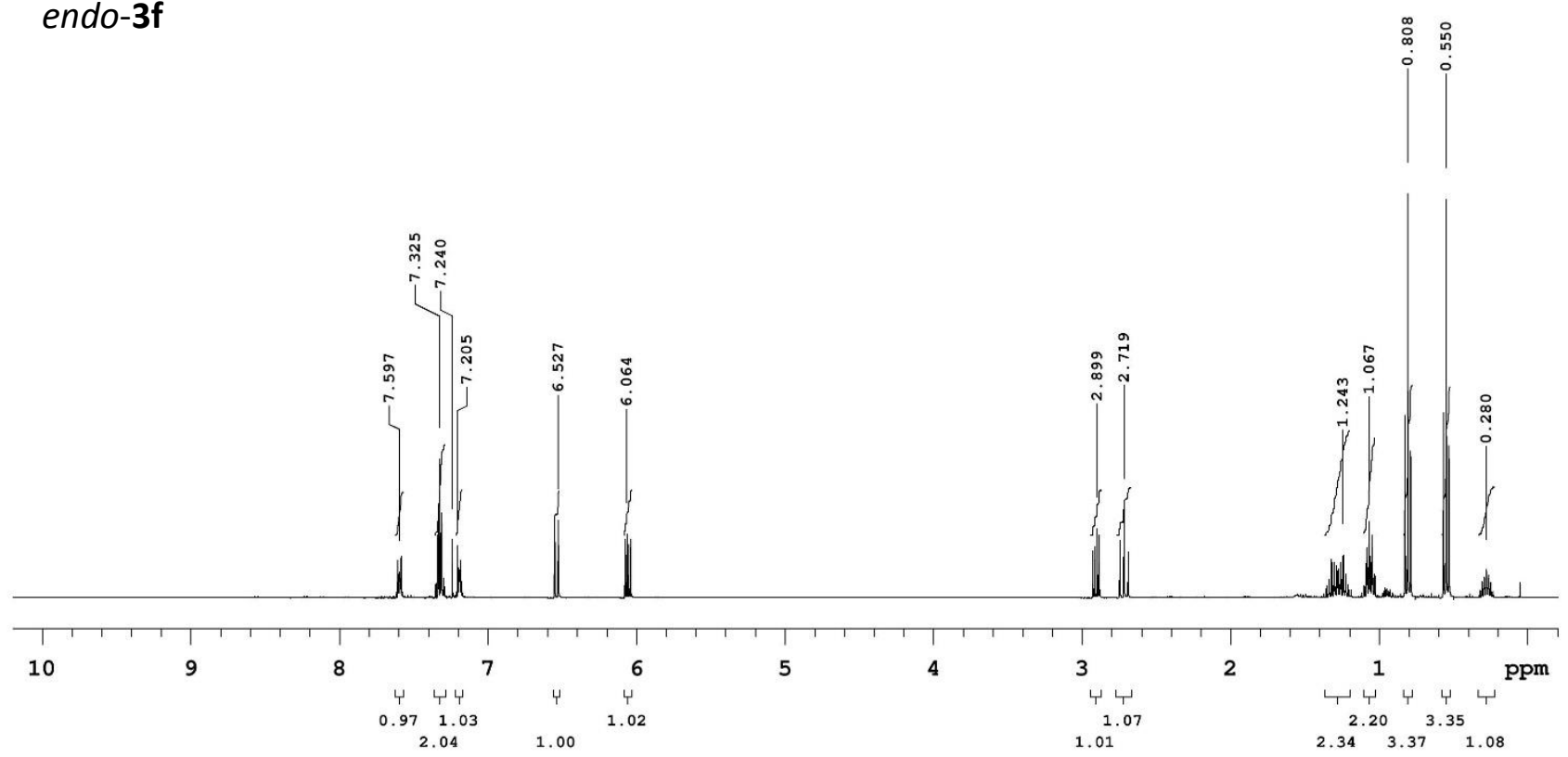

Sample Name: DA-237d

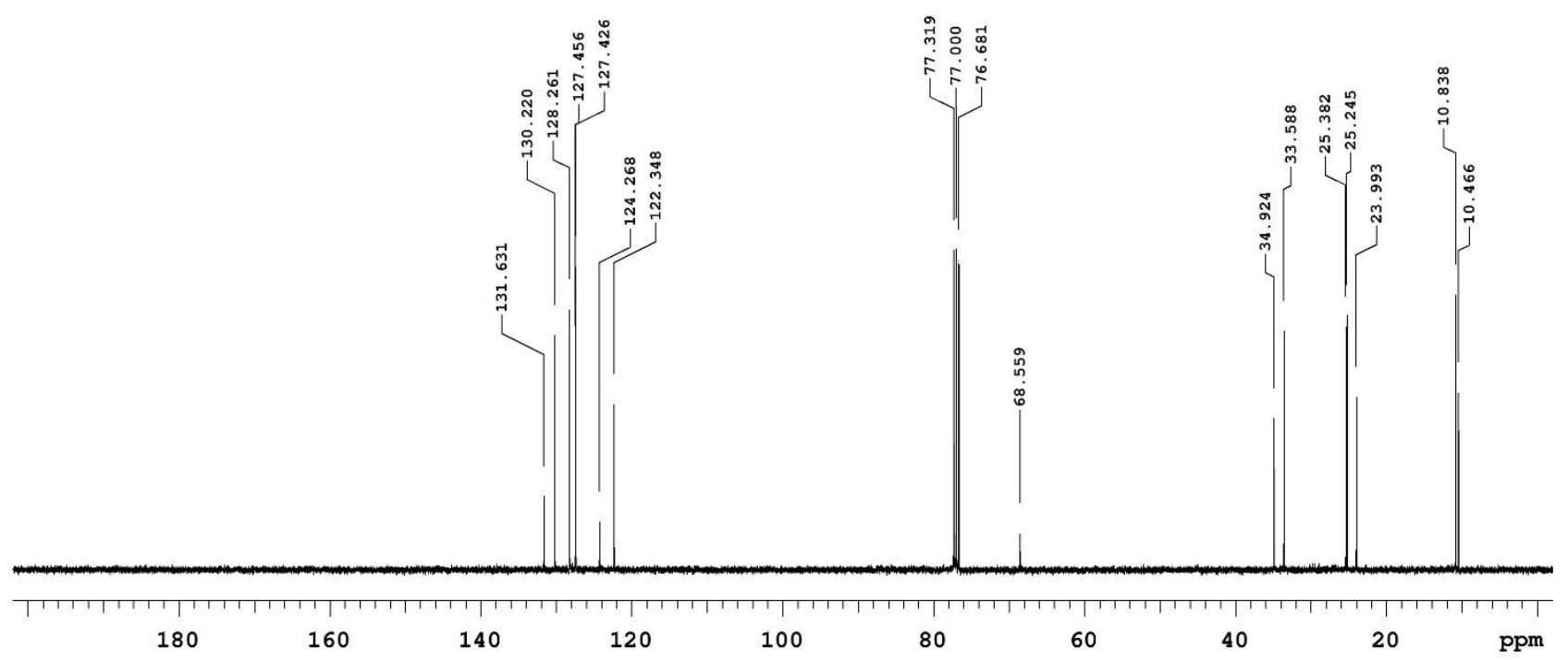


Sample Name: DA-236a
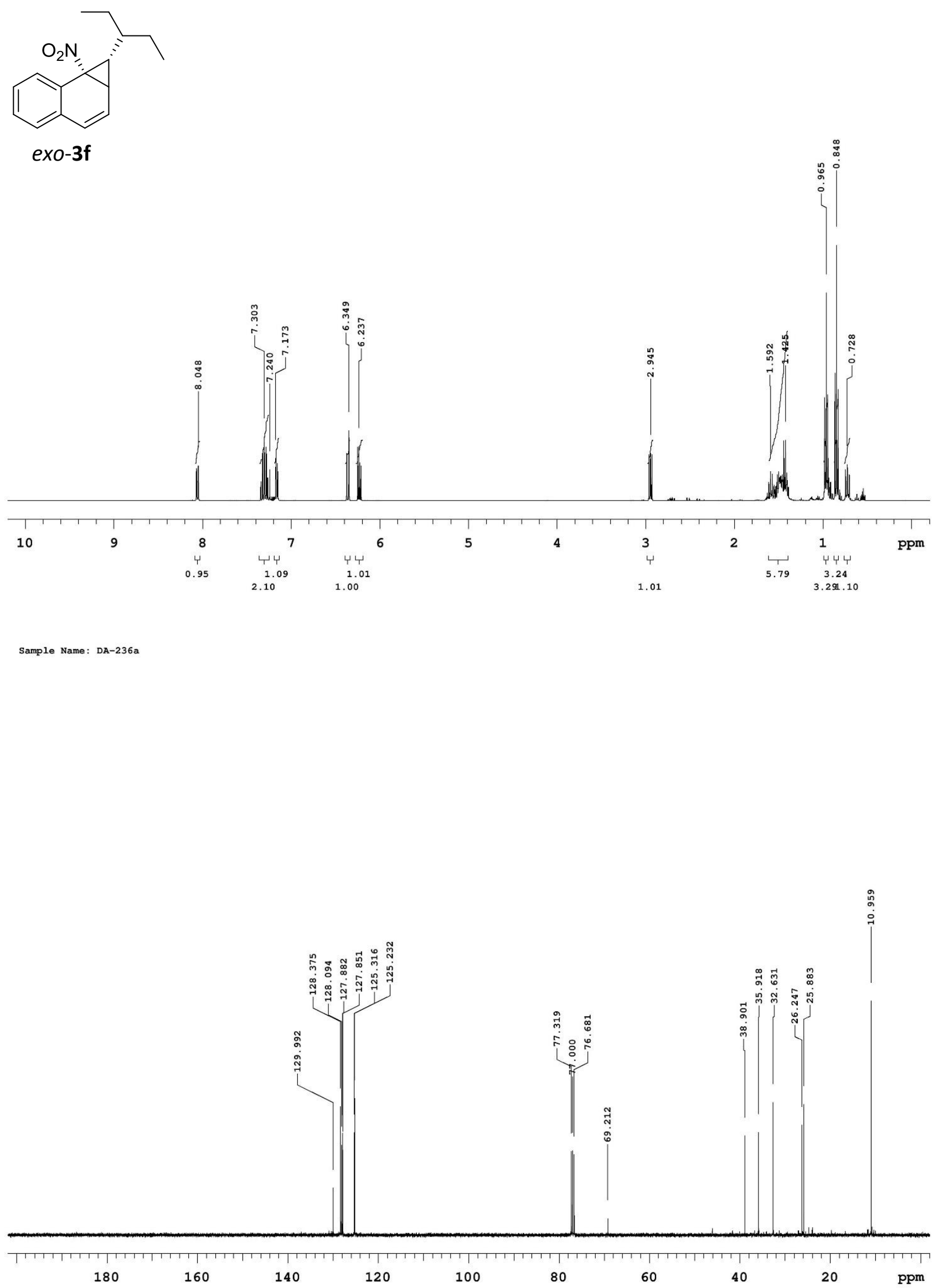
Sample Name: DA-208ae

Solvent: cdc13

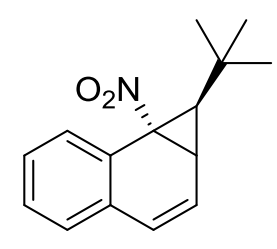

endo-3g

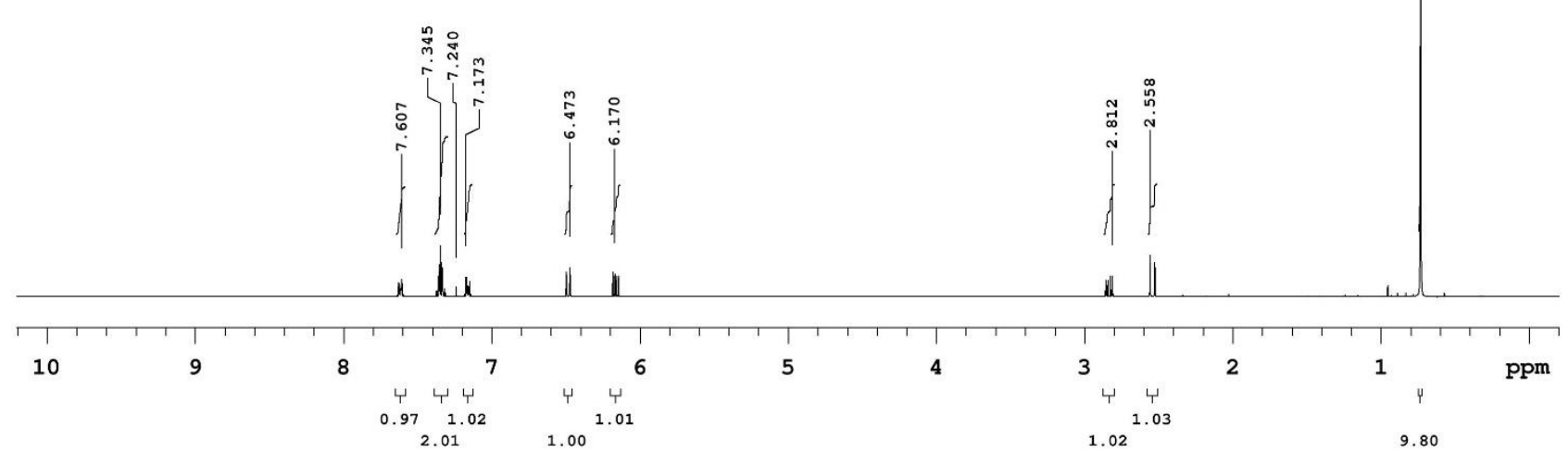

Sample Name: DA-208ae

Solvent: $\operatorname{cdcl} 3$

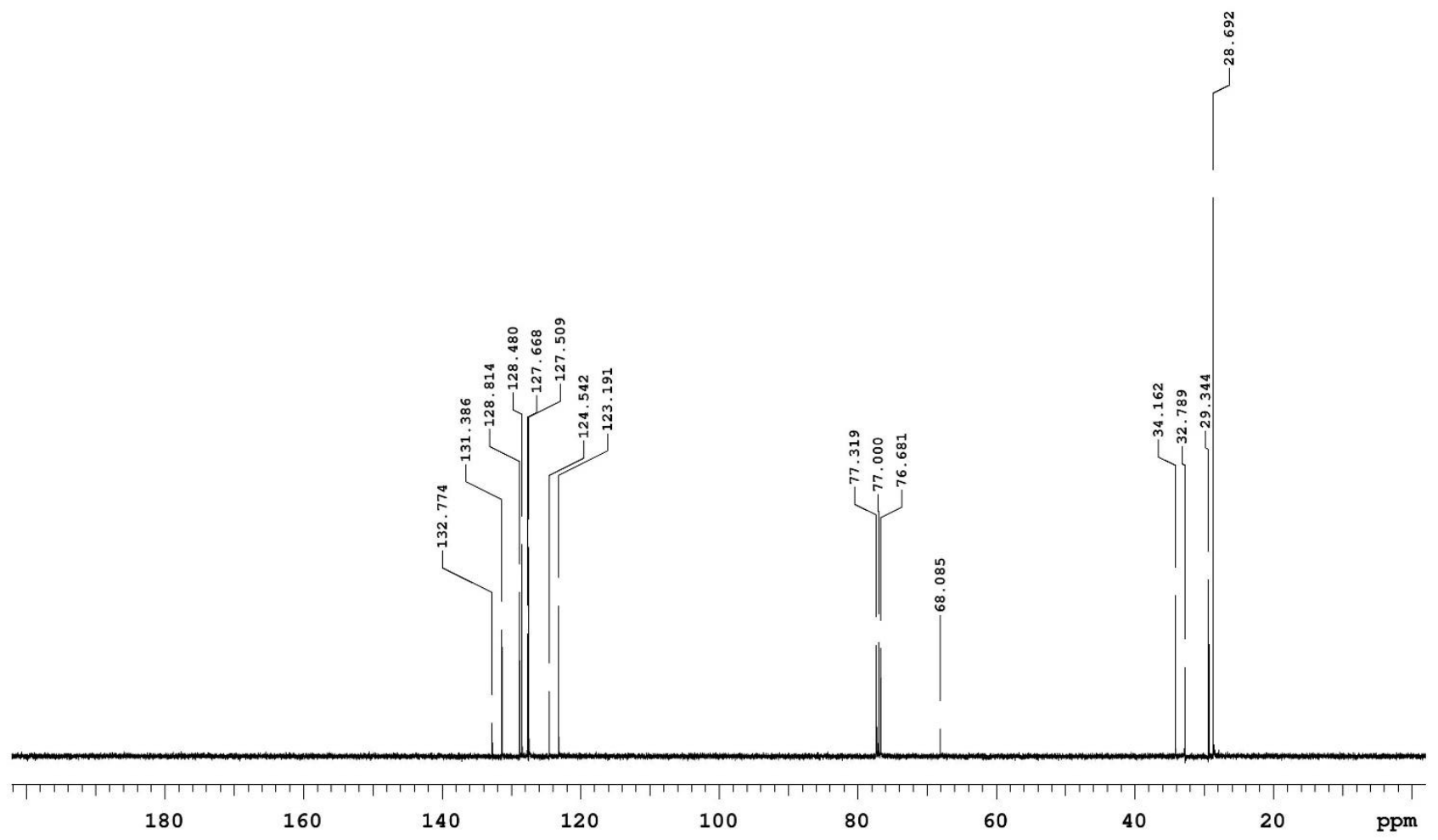


Sample Name: DA-264e

Solvent: cdcl3

(1)

endo-3h

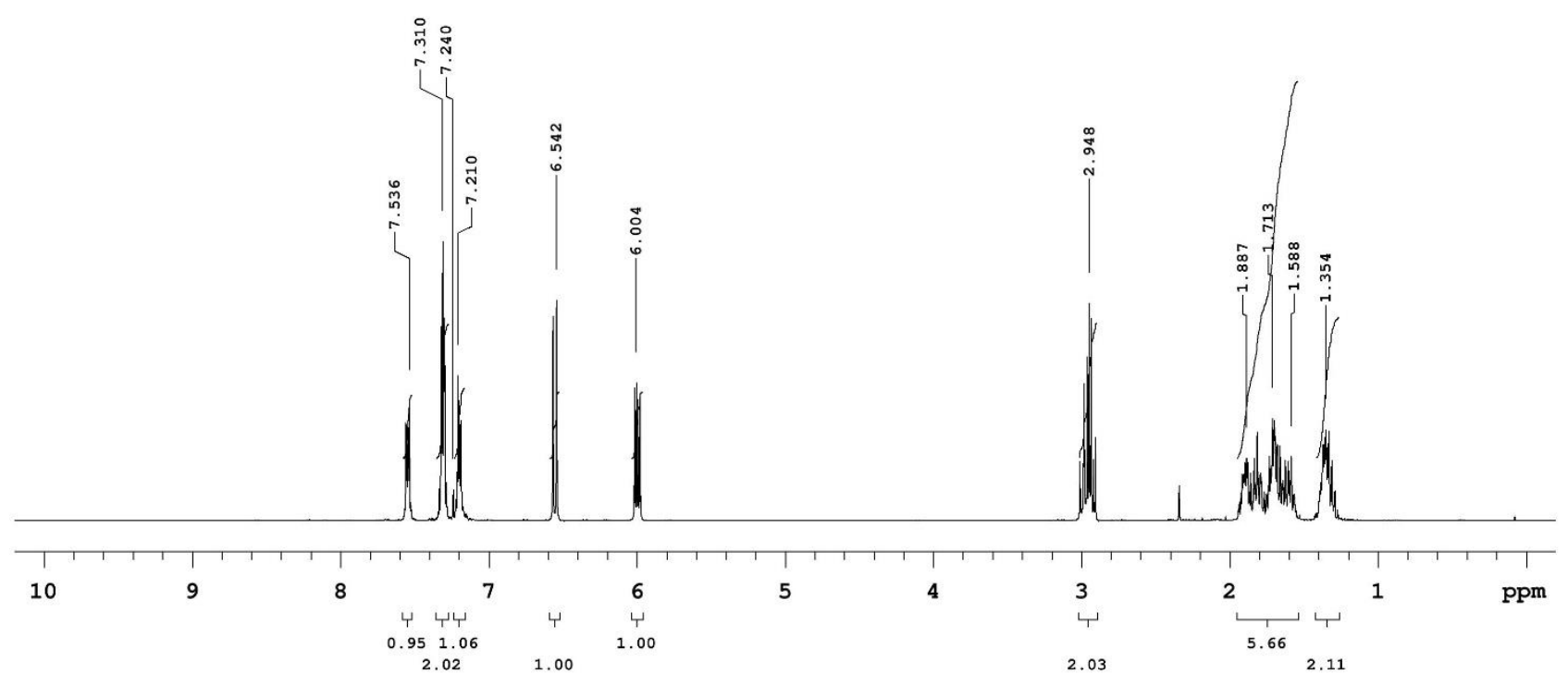

Sample Name: DA-264e

Solvent: cdc13

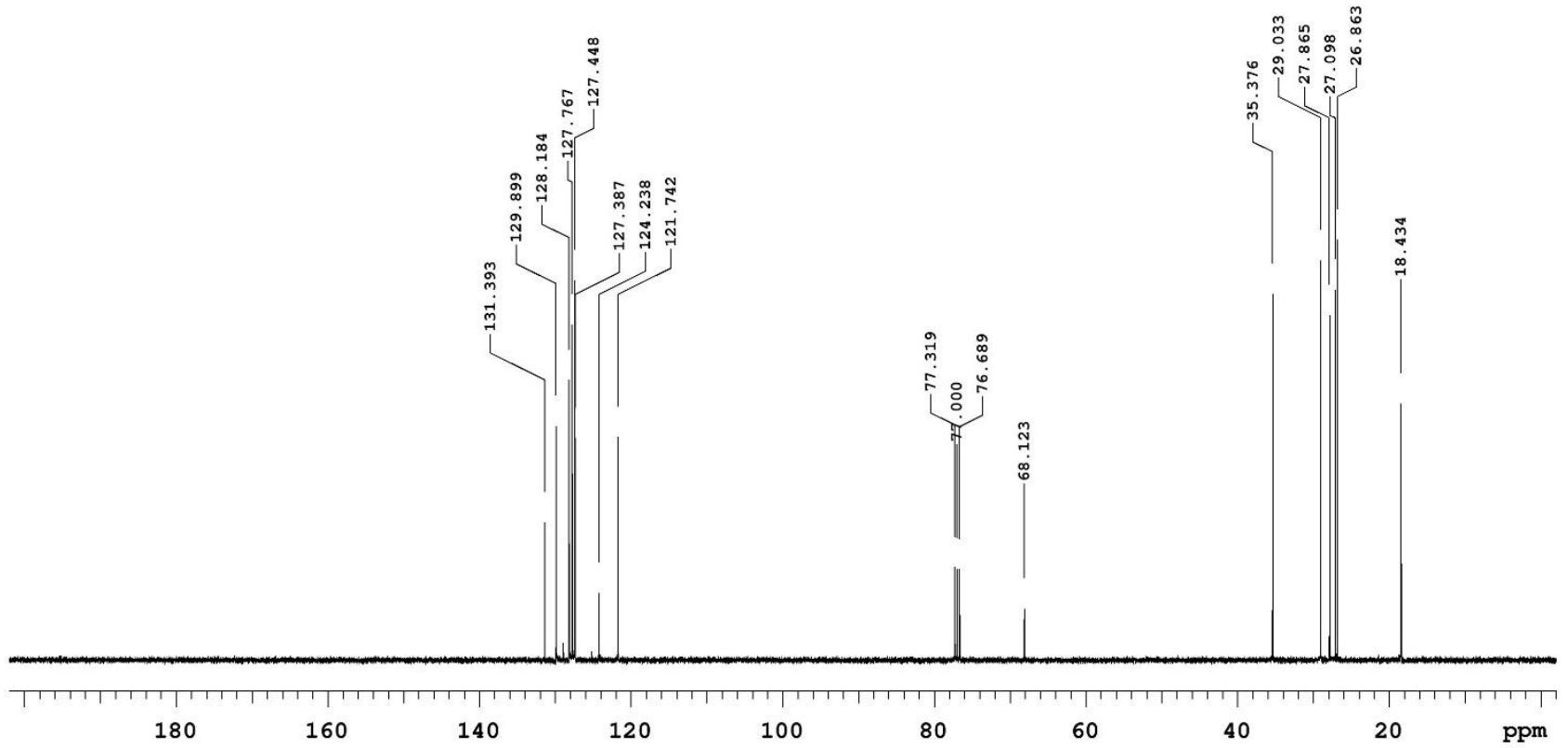


Sample Name: DA-264b

Solvent: cdcl3
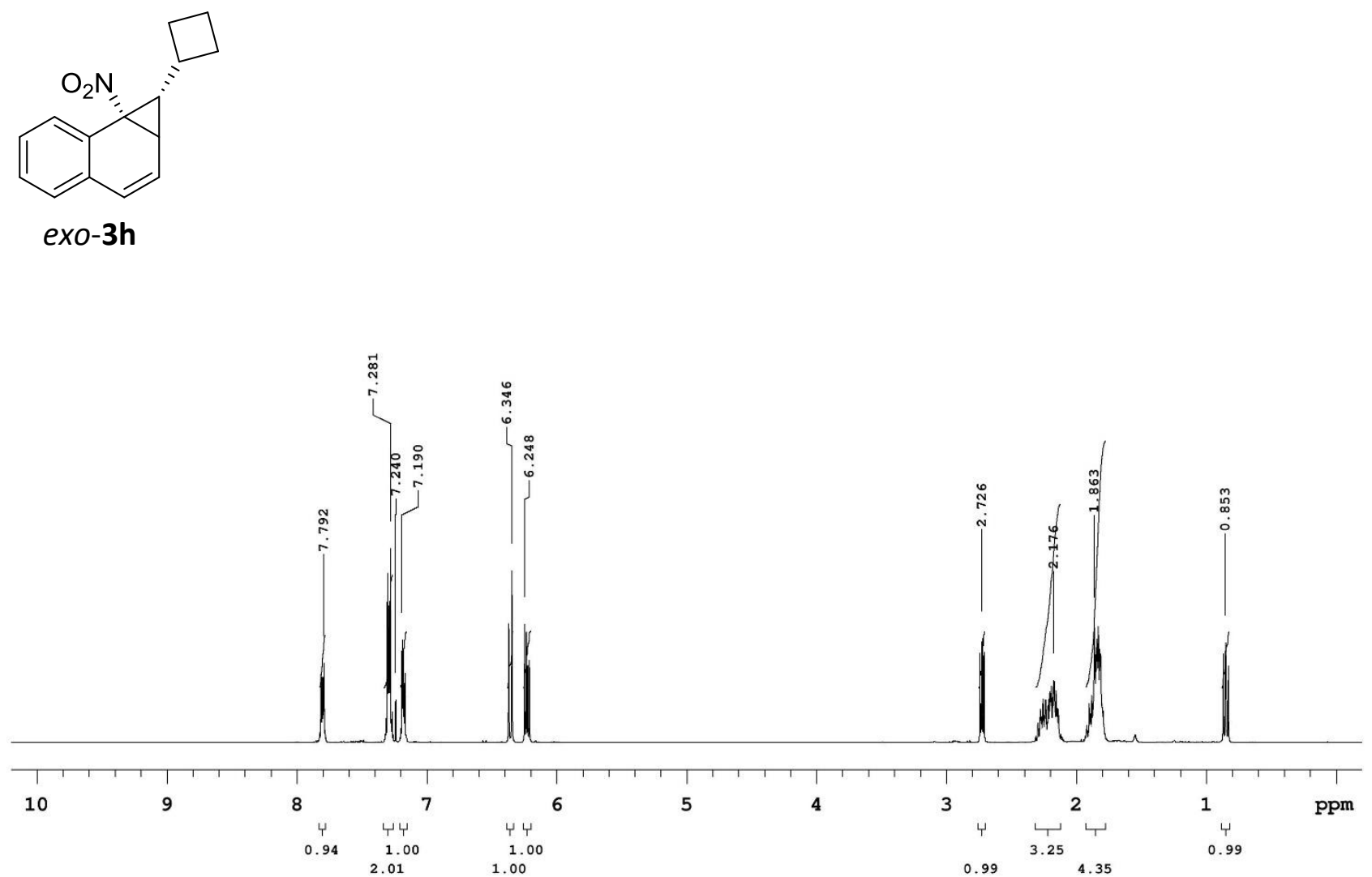

Sample Name: DA-264b

Solvent: cdc13

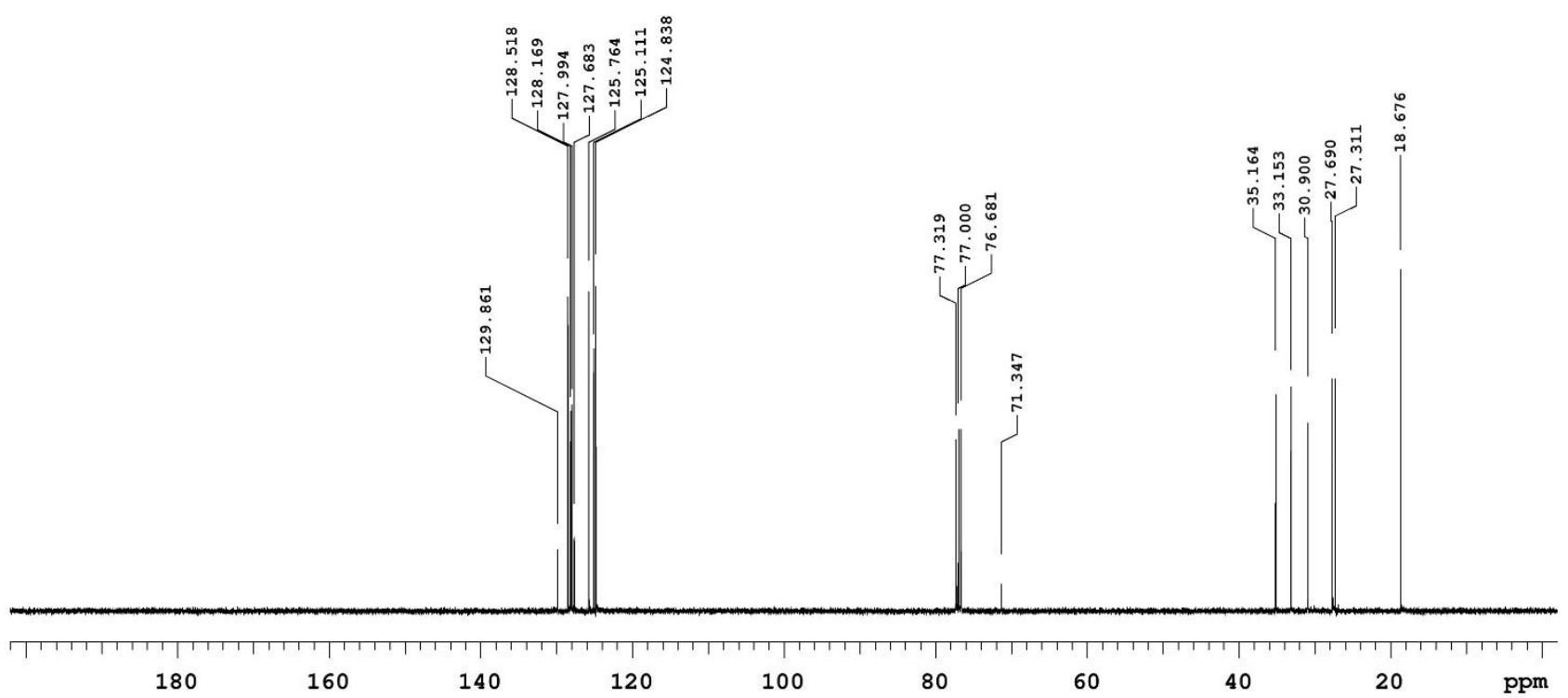


Sample Name: DA-234d-cryst

Solvent: cdc13

(O)

endo-3i

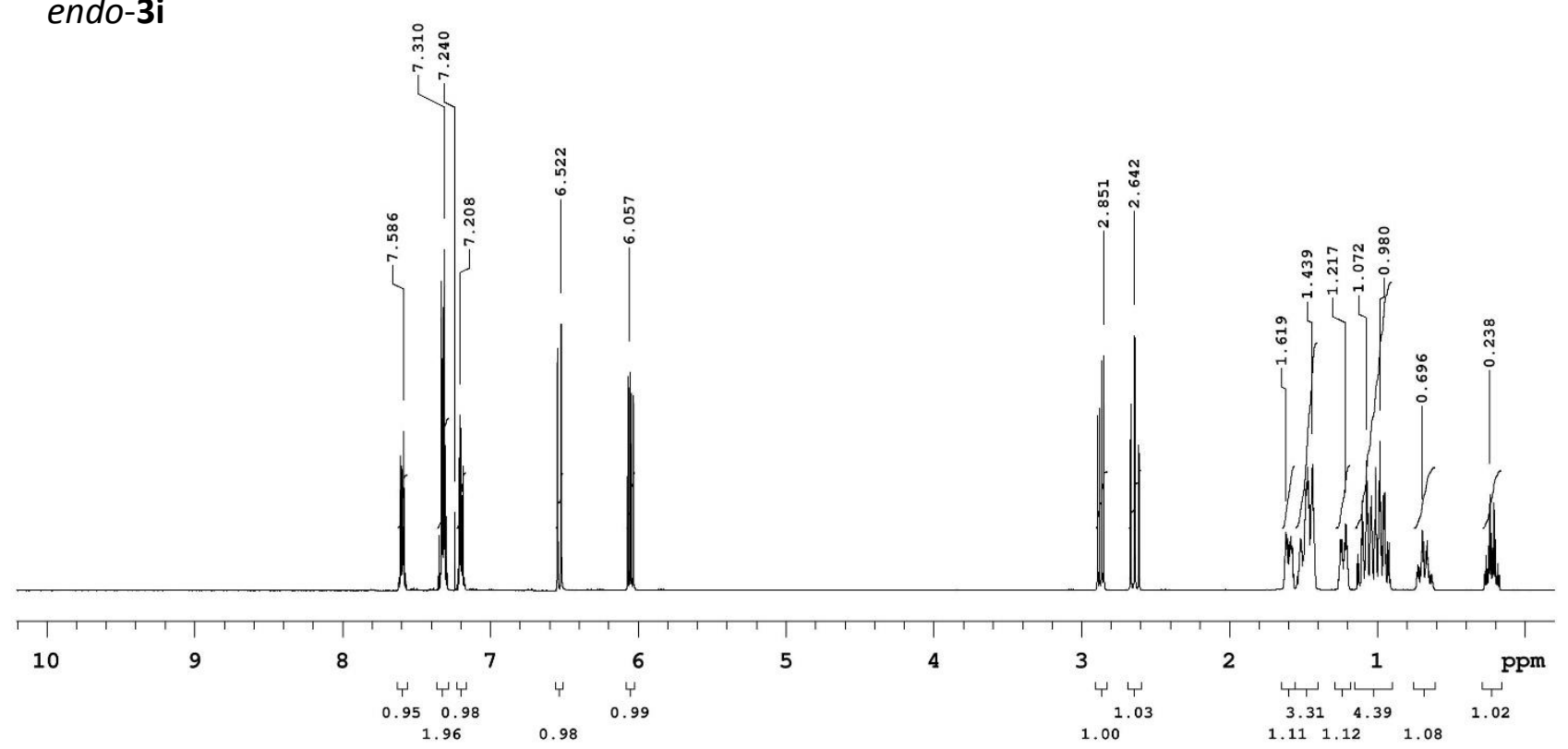

Sample Name: DA-234d-cryst

Solvent: cdc13

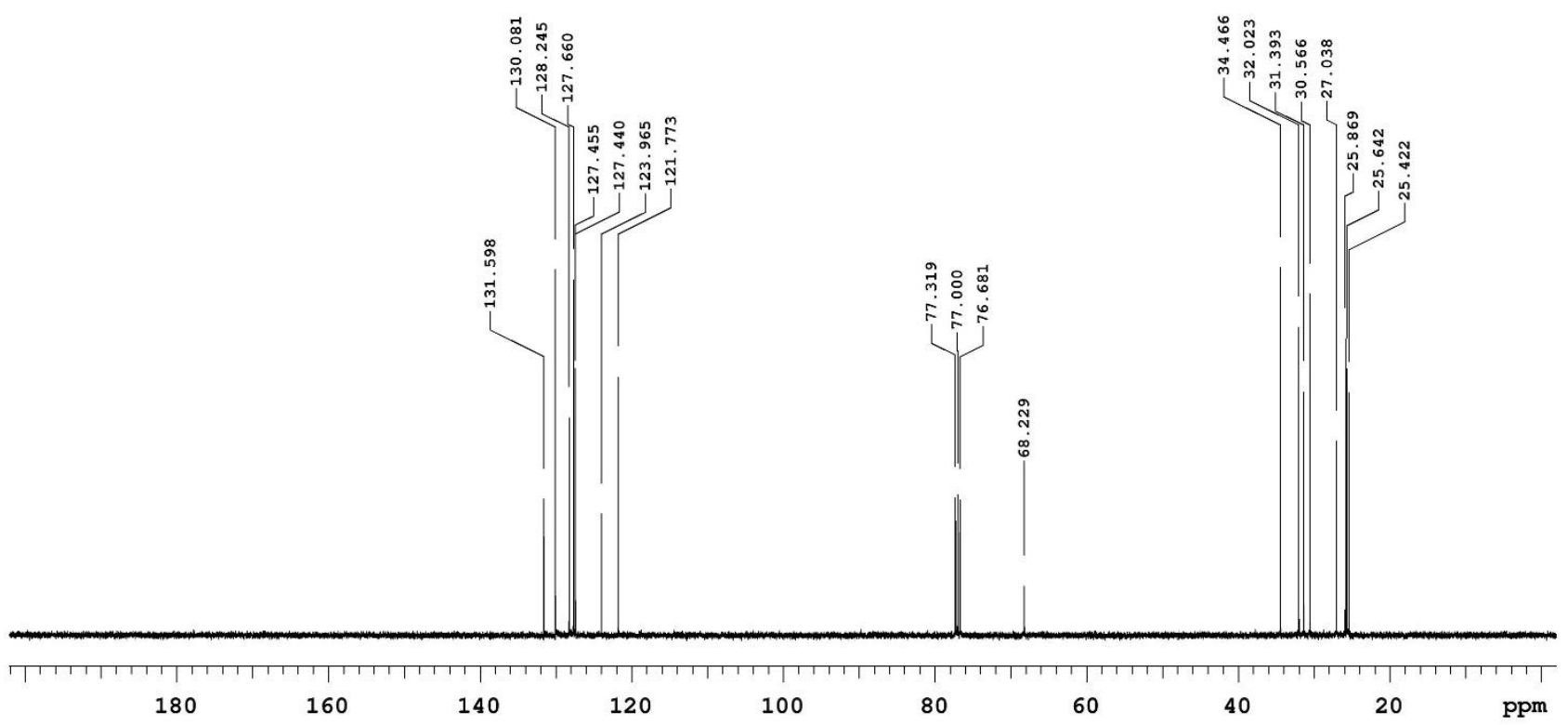


Sample Name: DA-234b

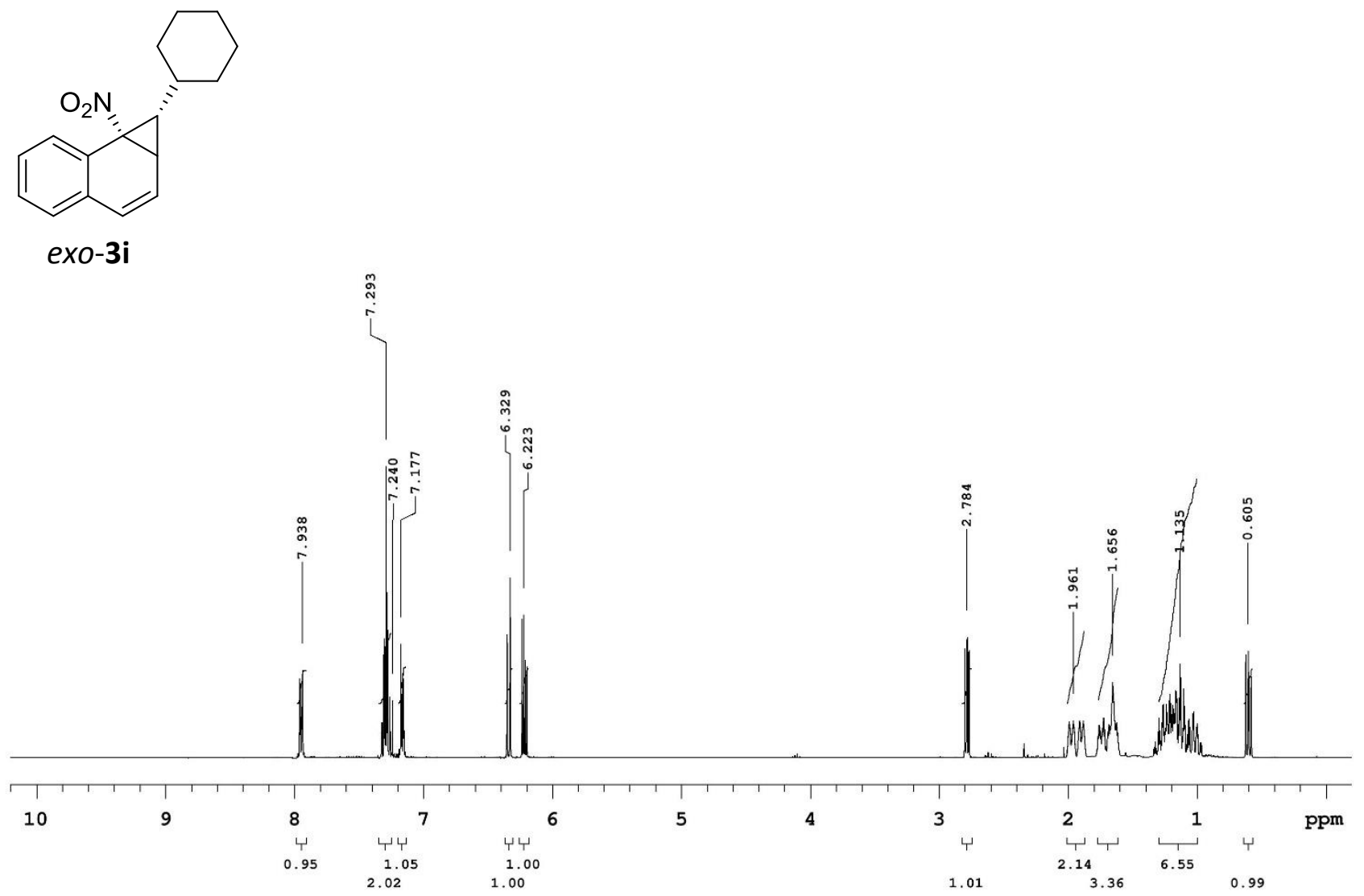

Sample Name: DA-234b

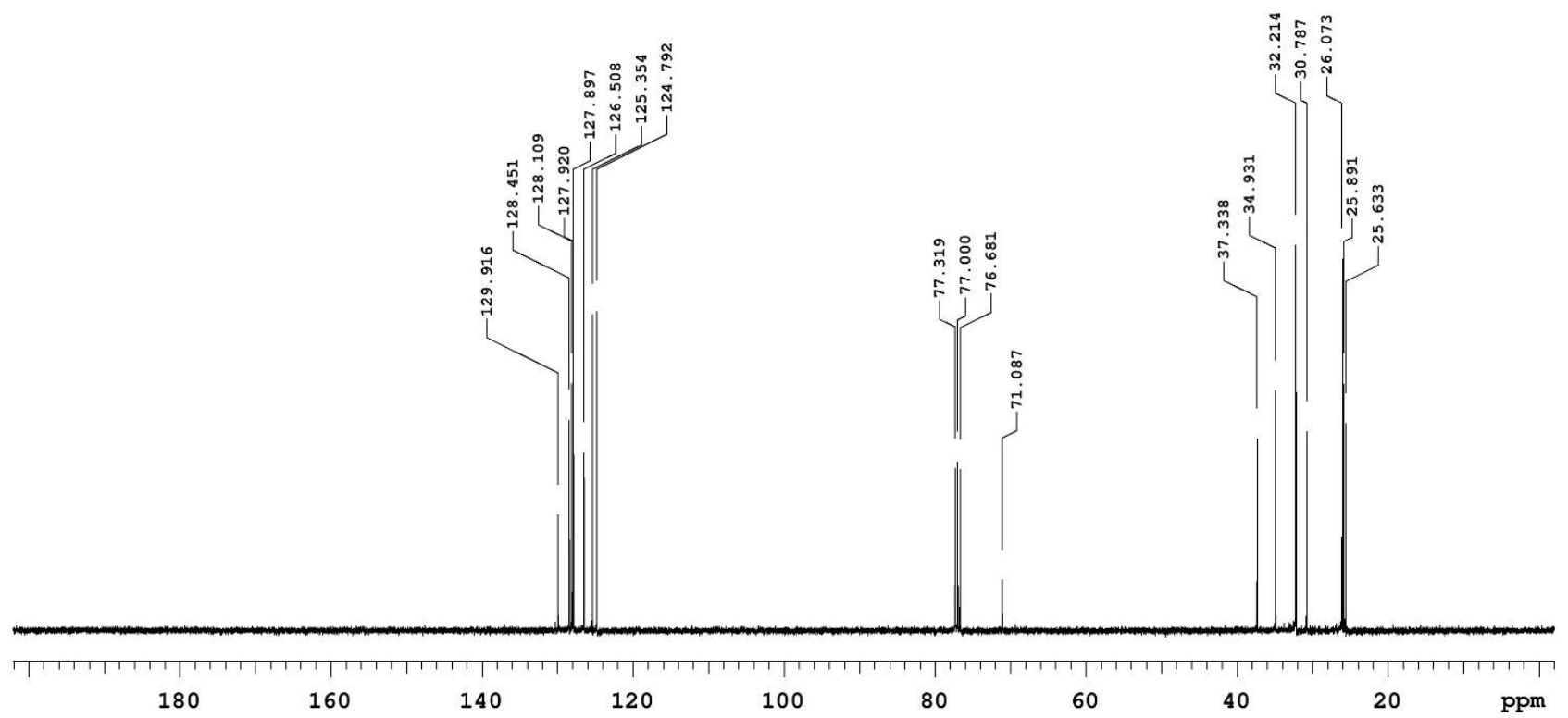




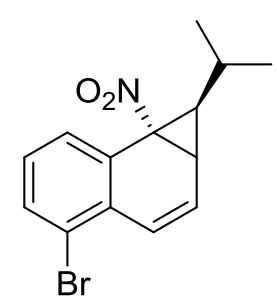

endo-3j
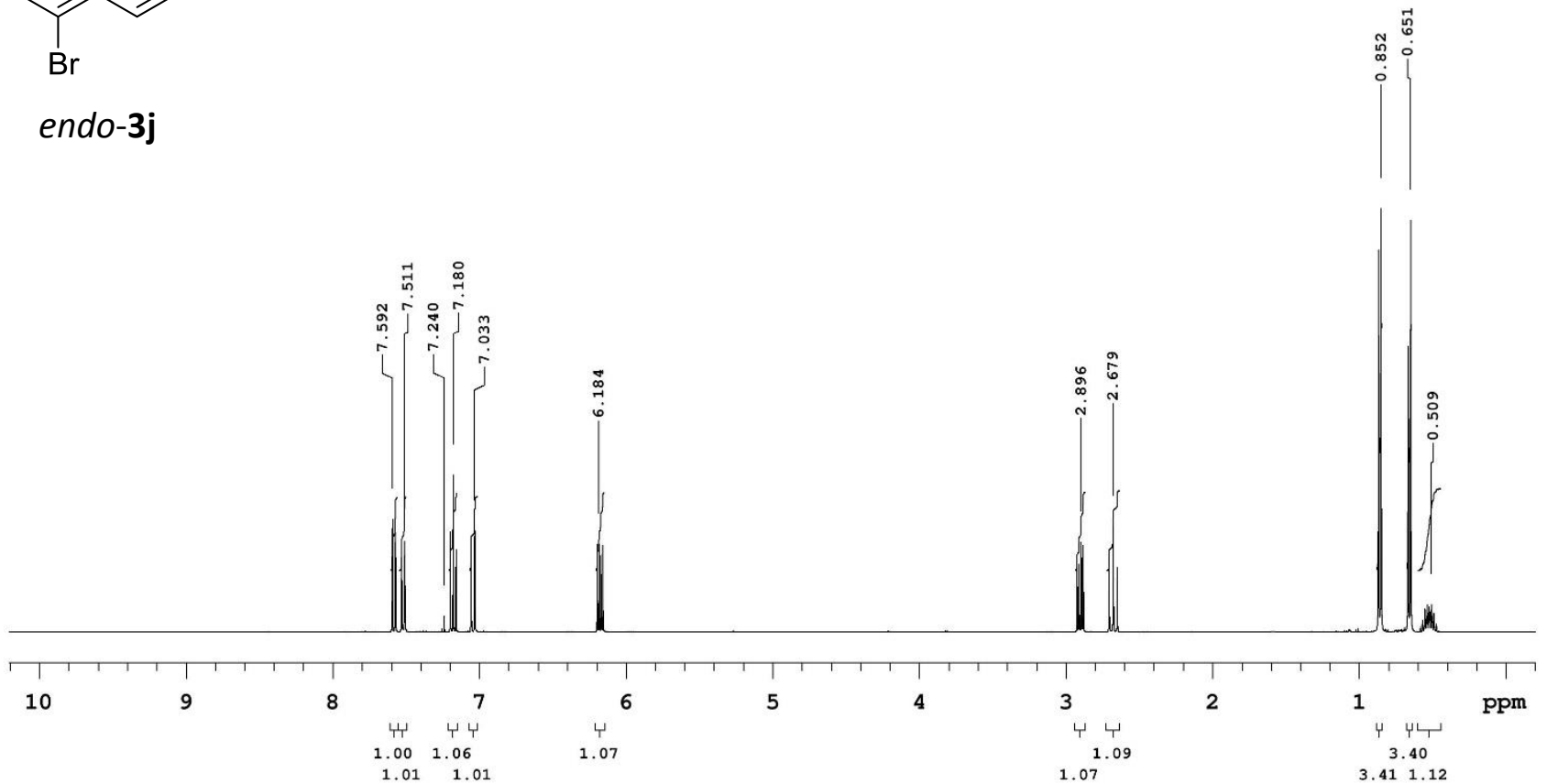

Sample Name: DA-192da-cryst Solvent: $\operatorname{cdcl} 3$

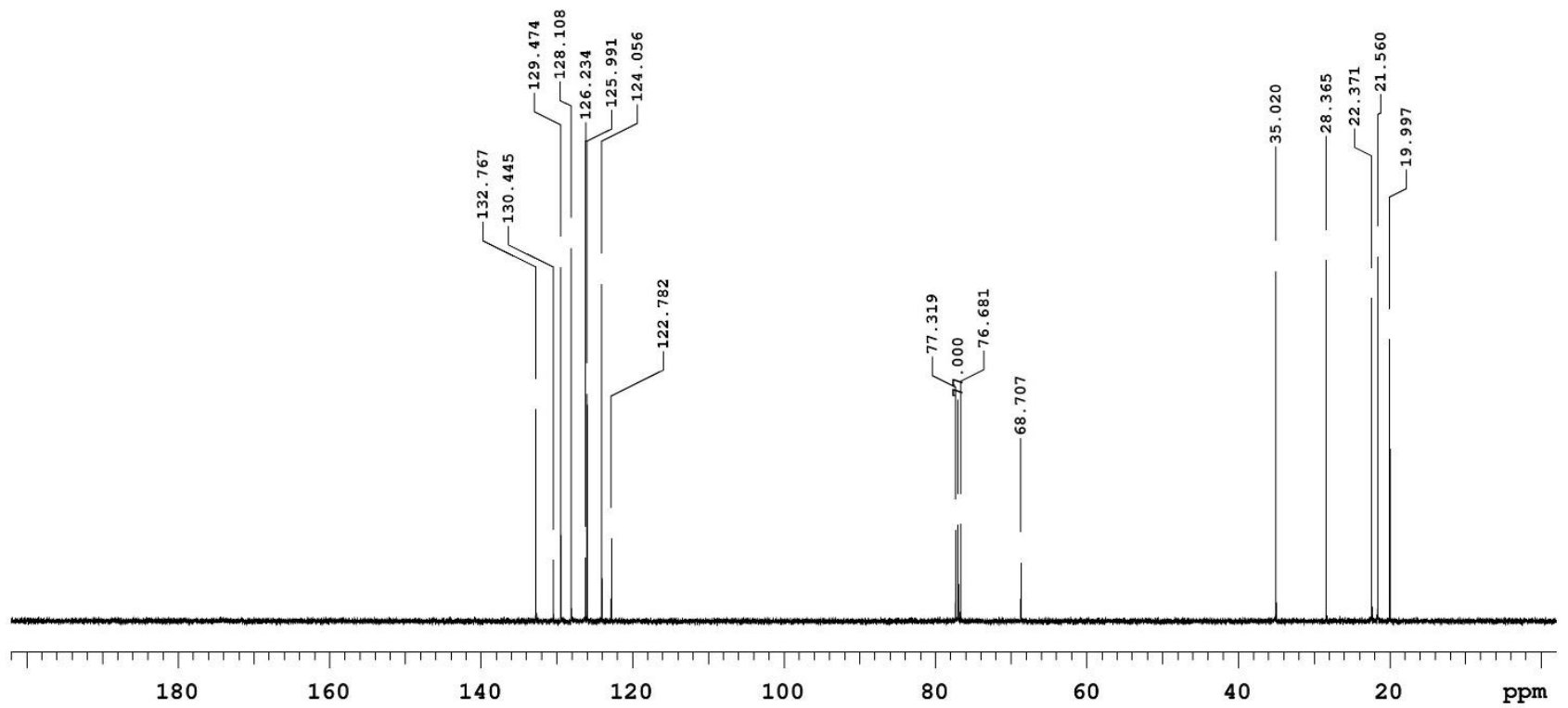


Sample Name: DA-192C

Solvent: $\operatorname{cdc} 13$

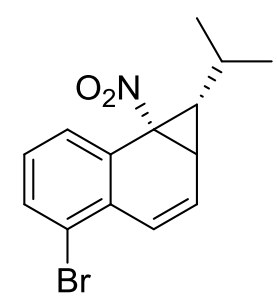

exo-3j

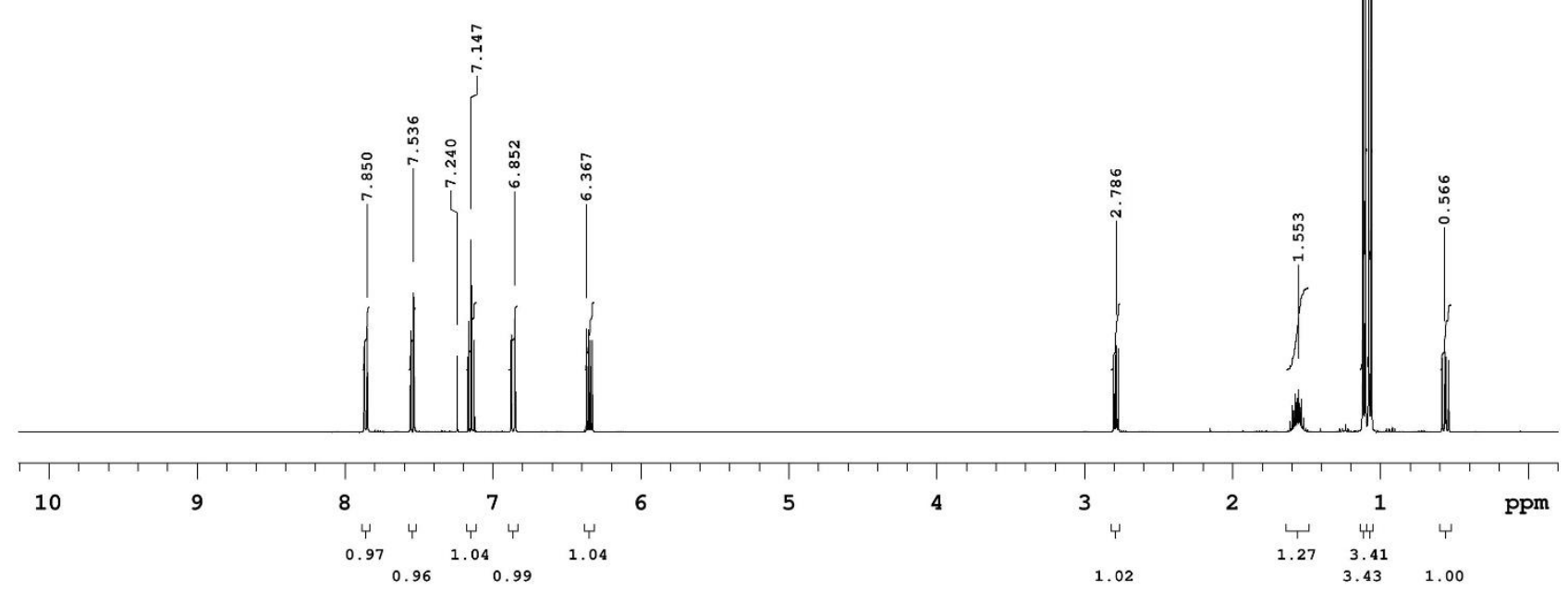

Sample Name: DA-192C

Solvent: cdc13

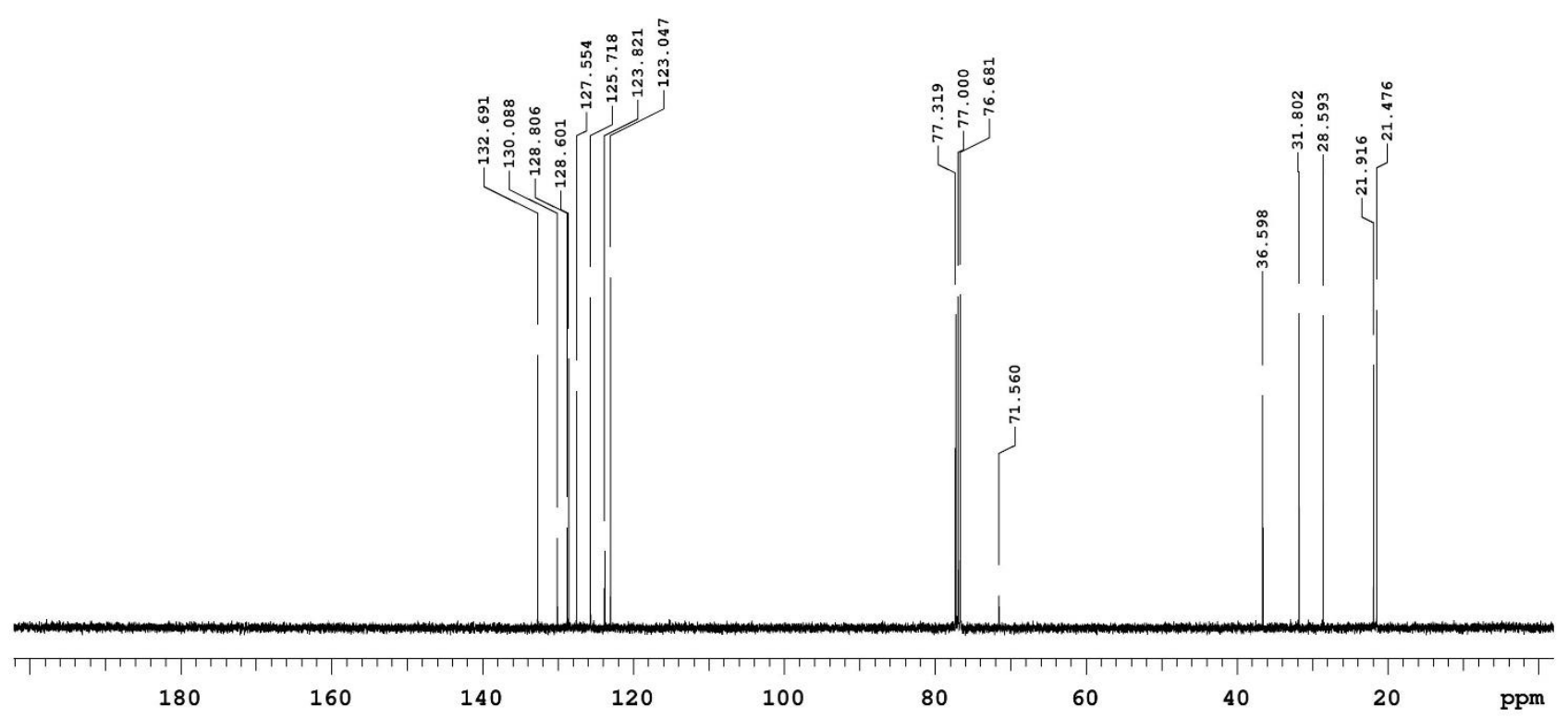




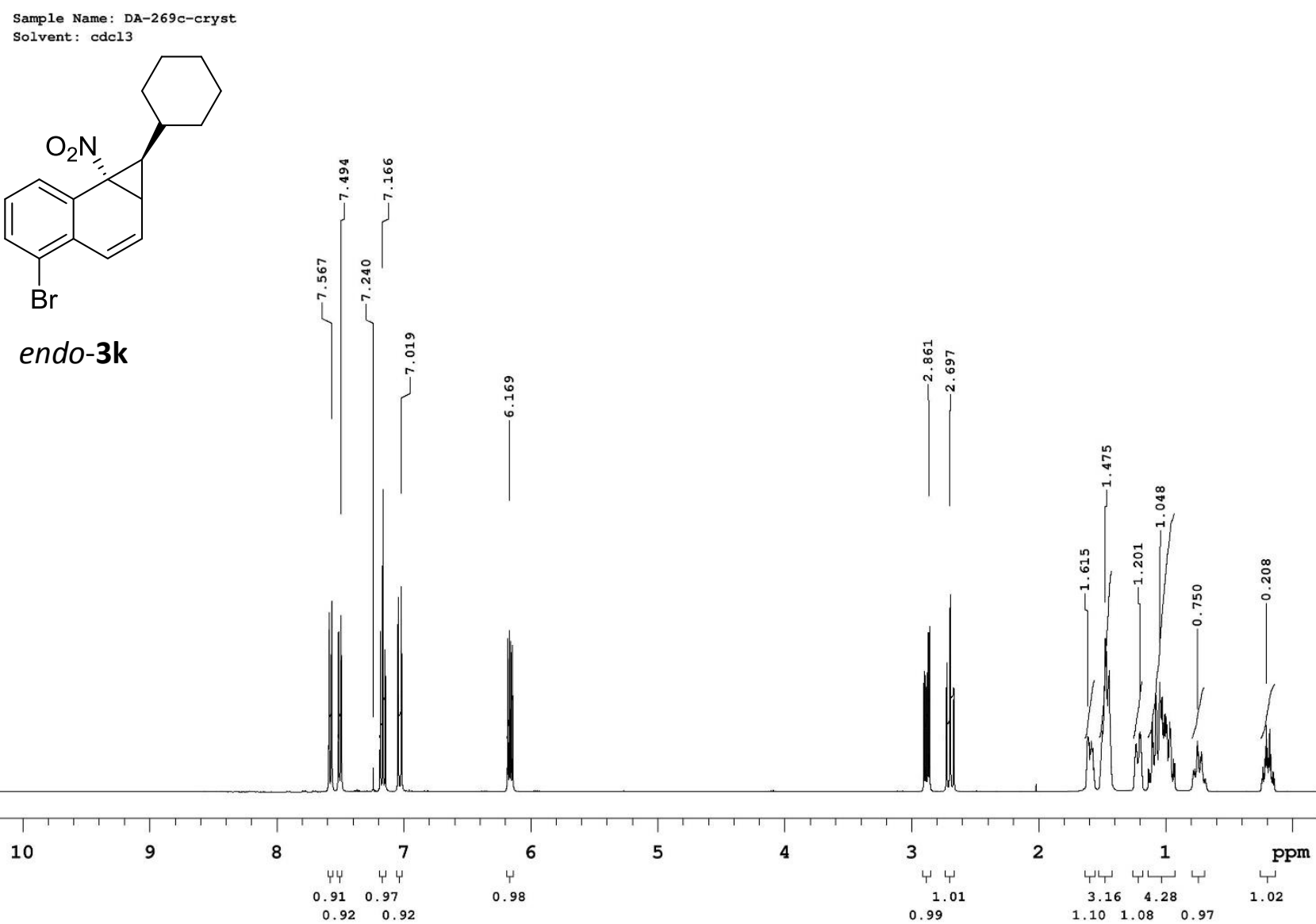

Sample Name: DA-269c-cryst Solvent: cdc13

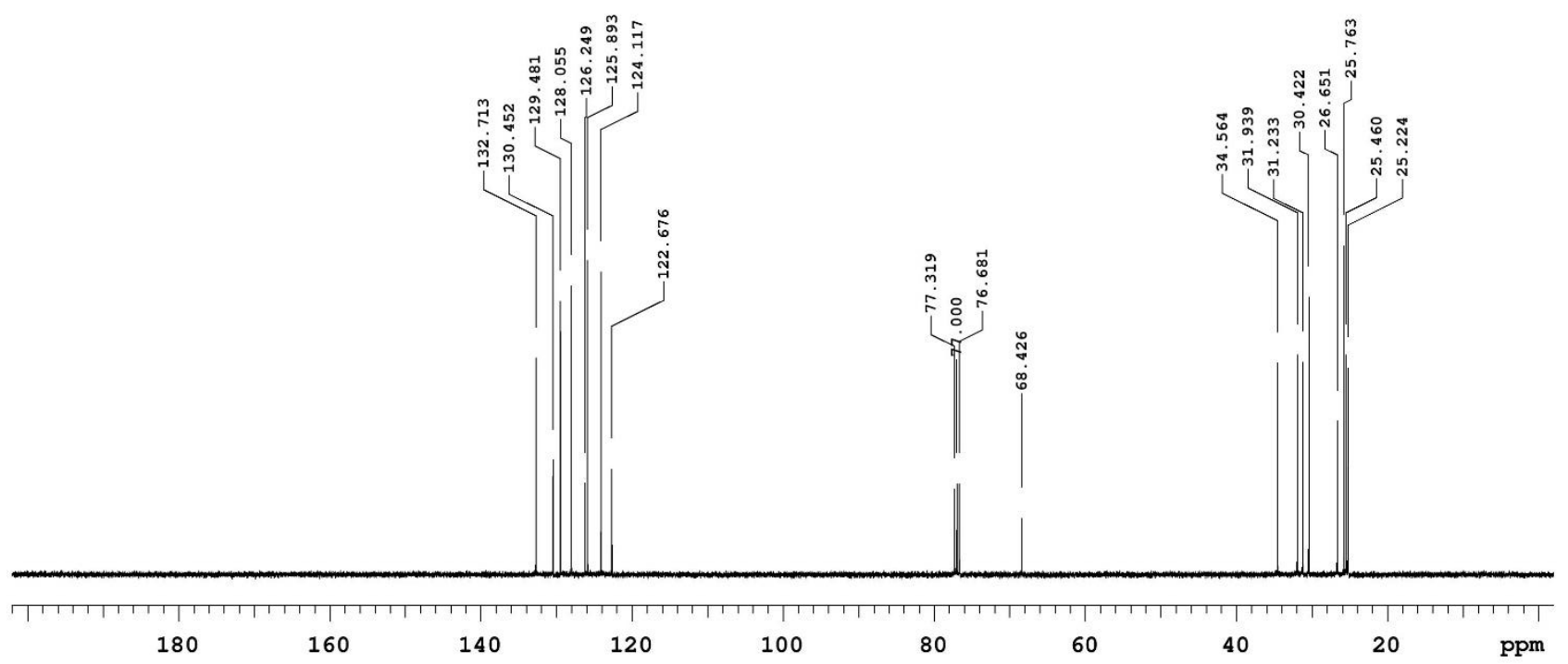




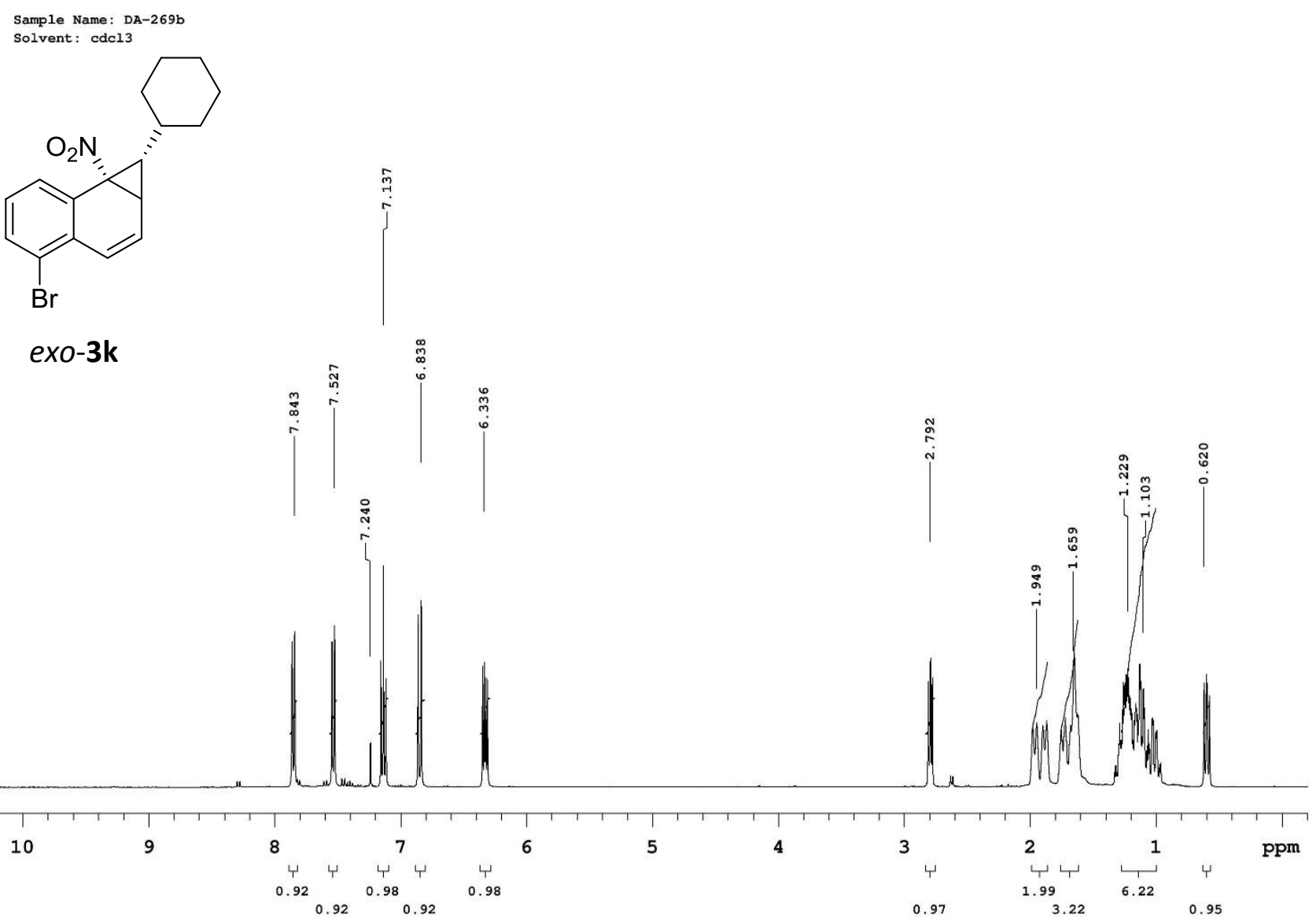

Sample Name: DA-269b Solvent: cdcl3

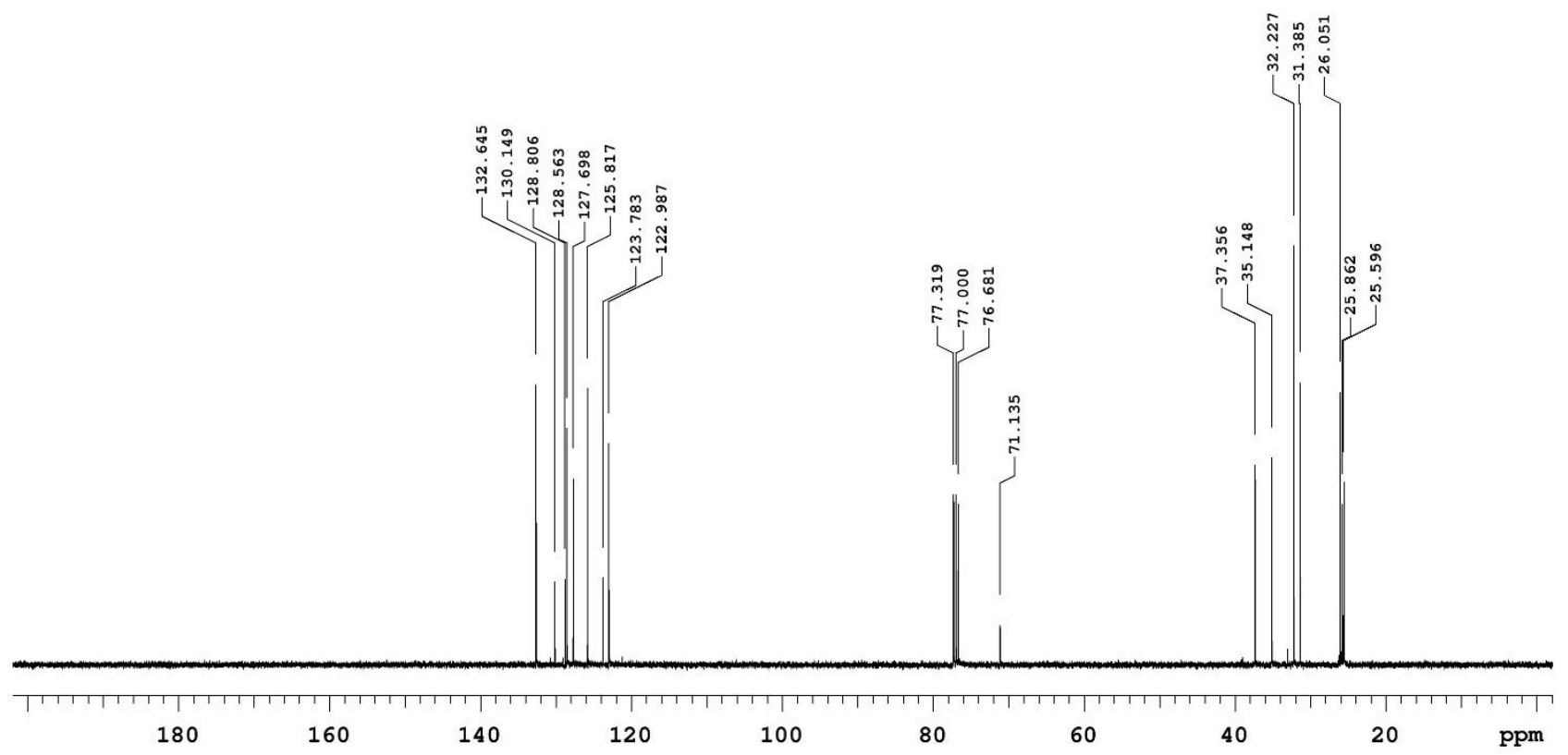


Sample Name: DA-254C

Solvent: cdc13
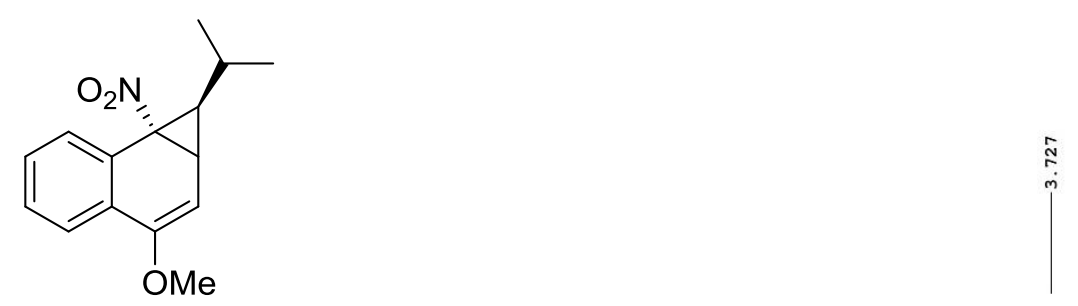

endo-3|

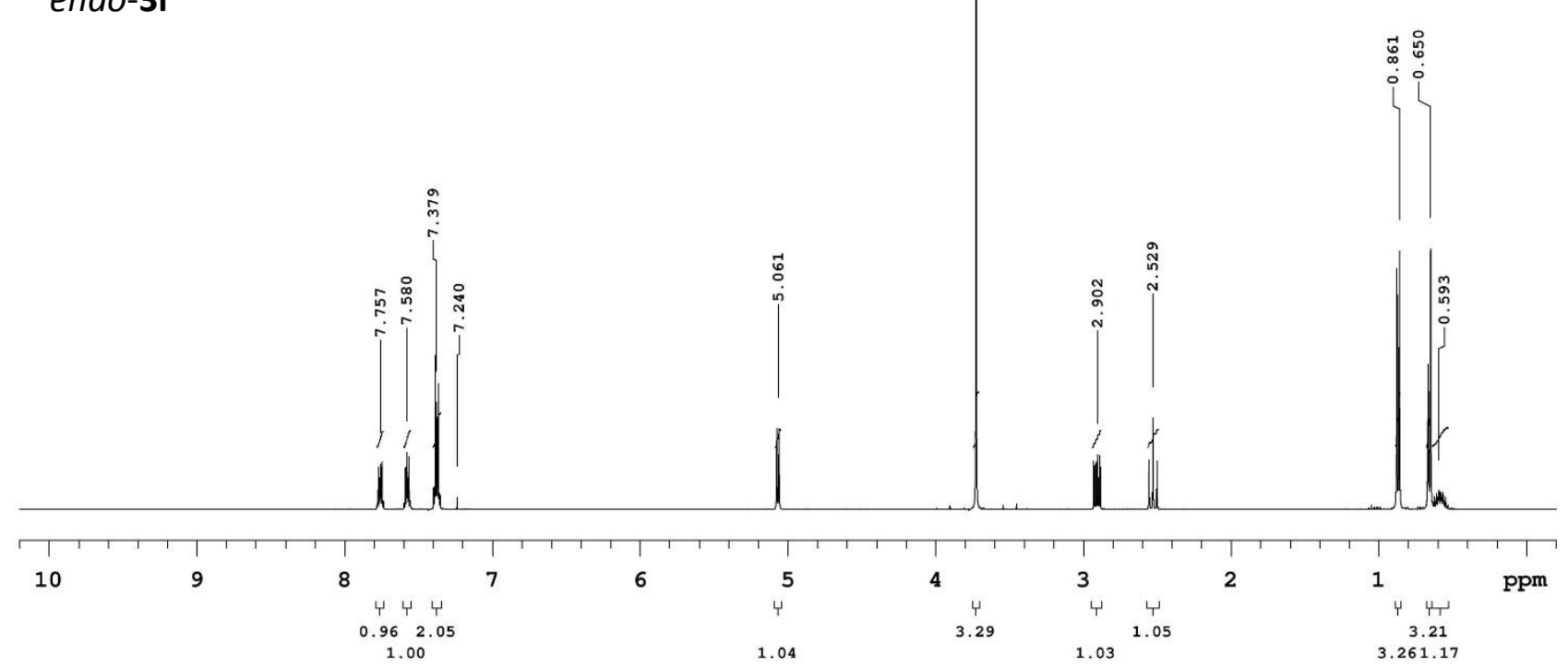

Sample Name: DA-254c

Solvent: cdc13

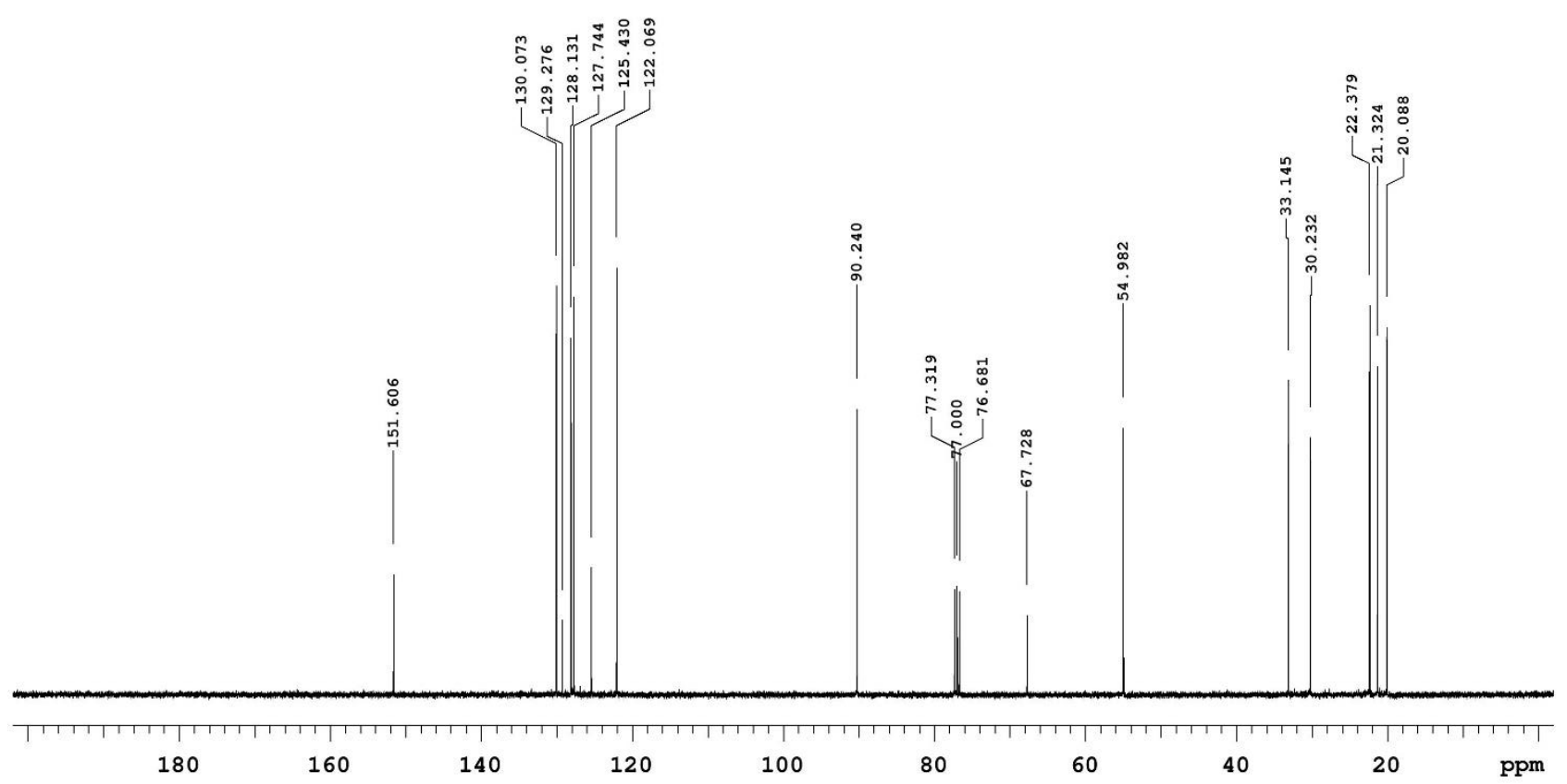


Sample Name: DA-274b

Solvent: cdc13

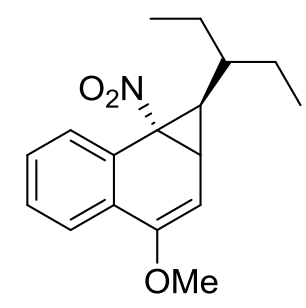

$\stackrel{\substack{\mathfrak{n} \\ \mathfrak{m}}}{\mid}$

endo-3m

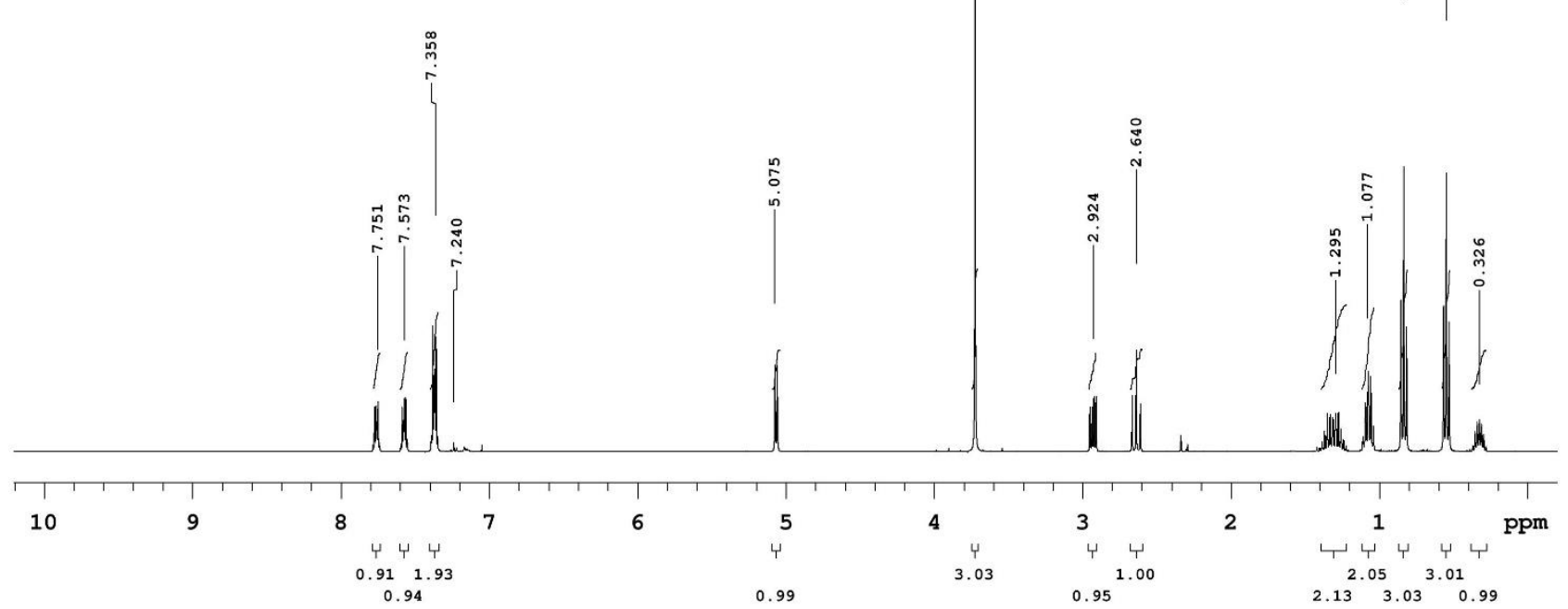

Sample Name: DA-274b

Solvent: cdc13

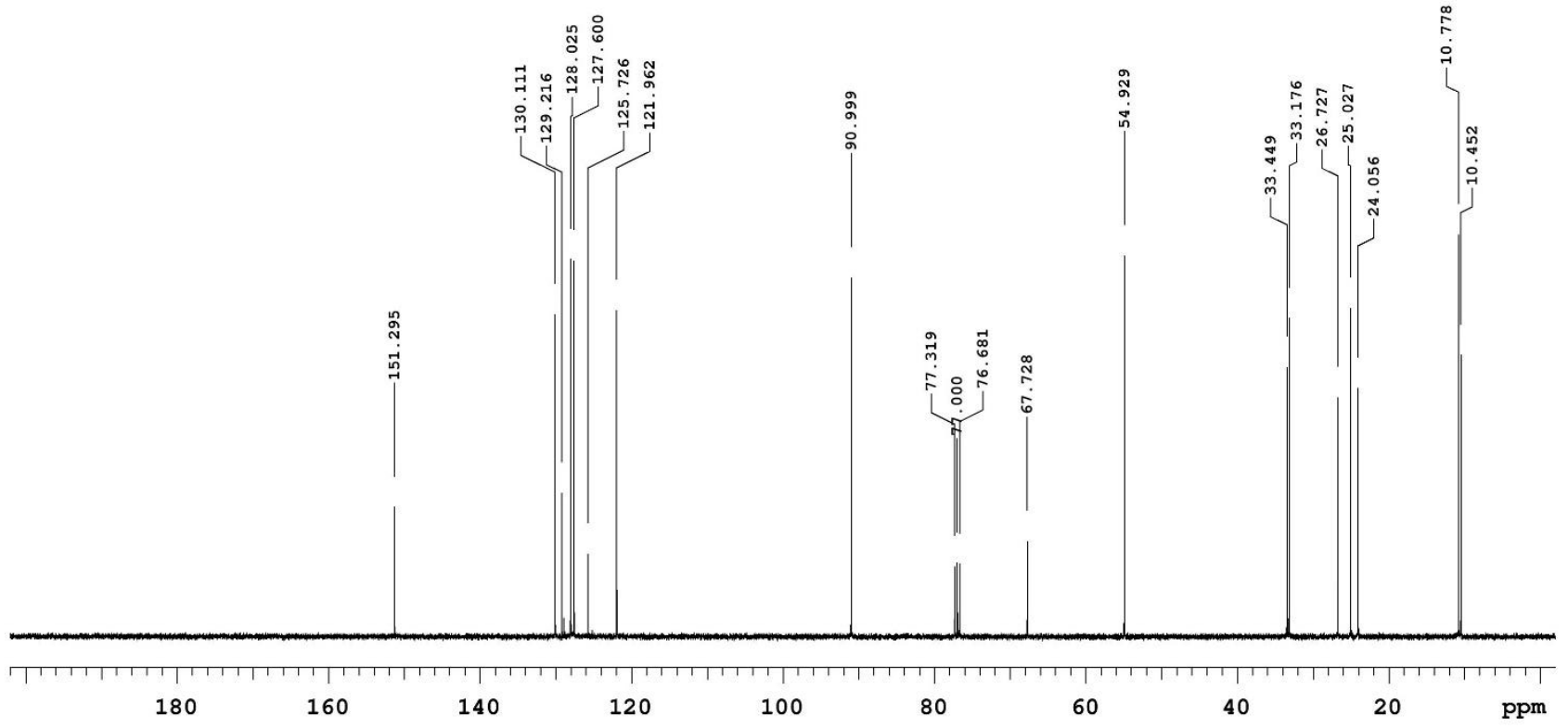


Sample Name: DA-274a

Solvent: cdc13

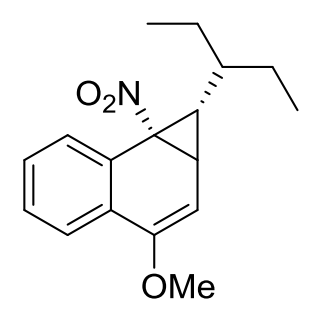

$\stackrel{\vec{p}}{\dot{p}}$

exo-3m

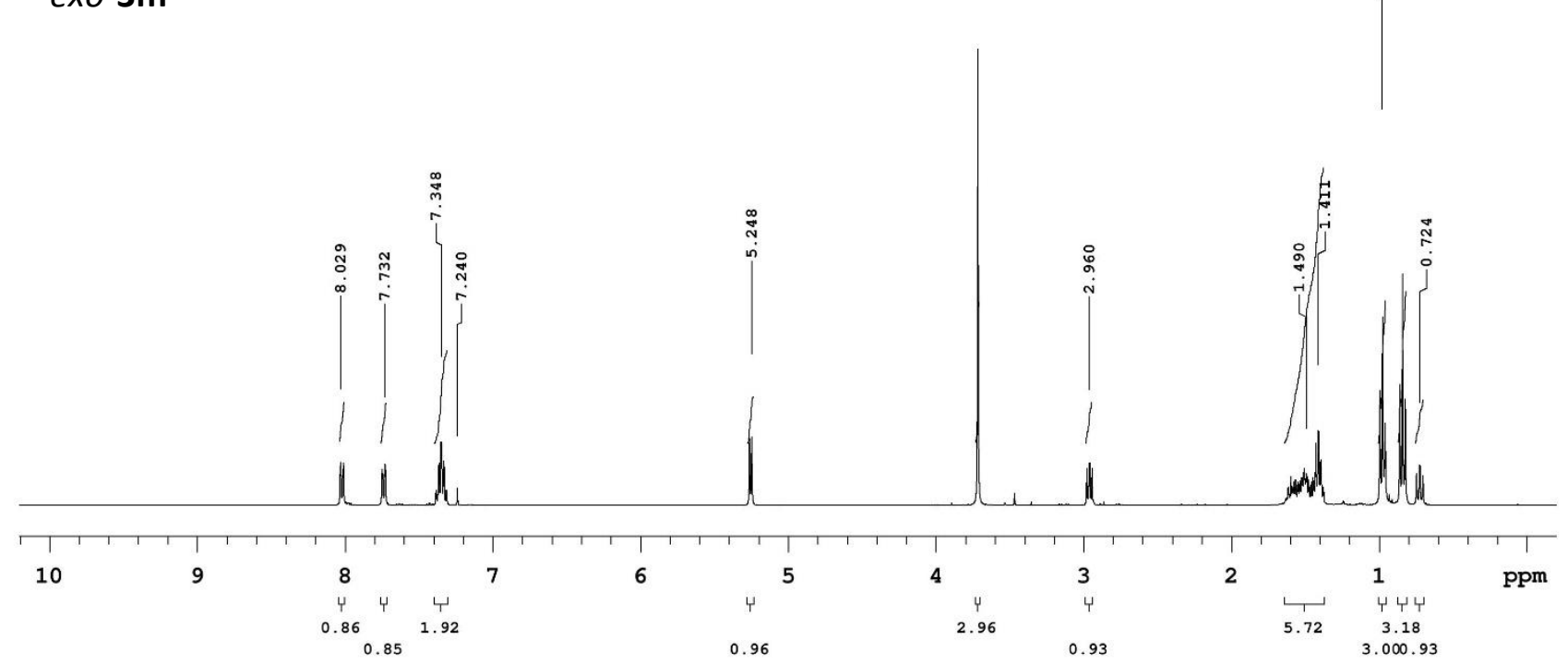

Sample Name: DA-274a

Solvent: cdc13

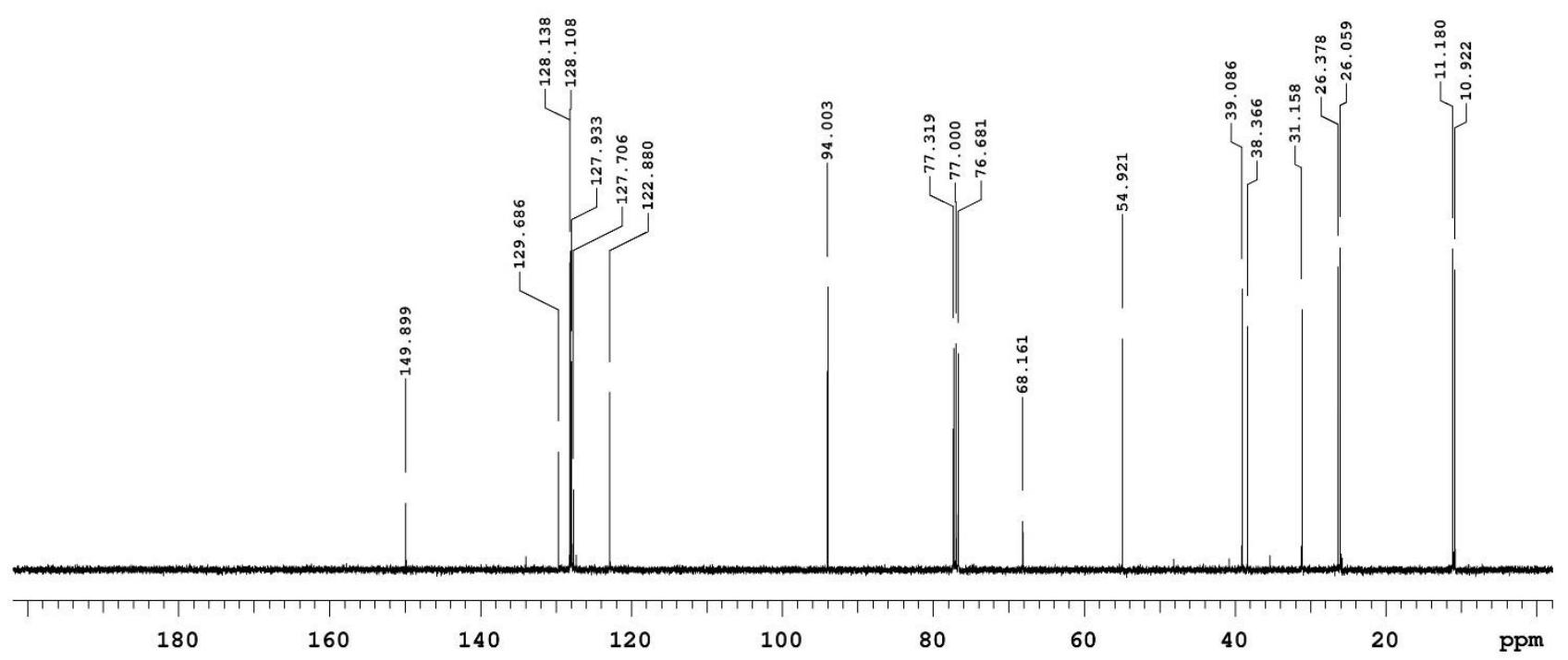


Sample Name: DA-275c

Solvent: cdc13

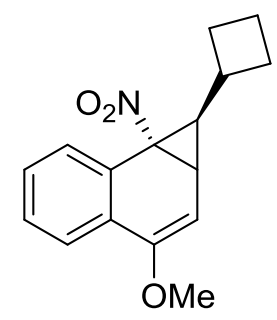

endo-3n
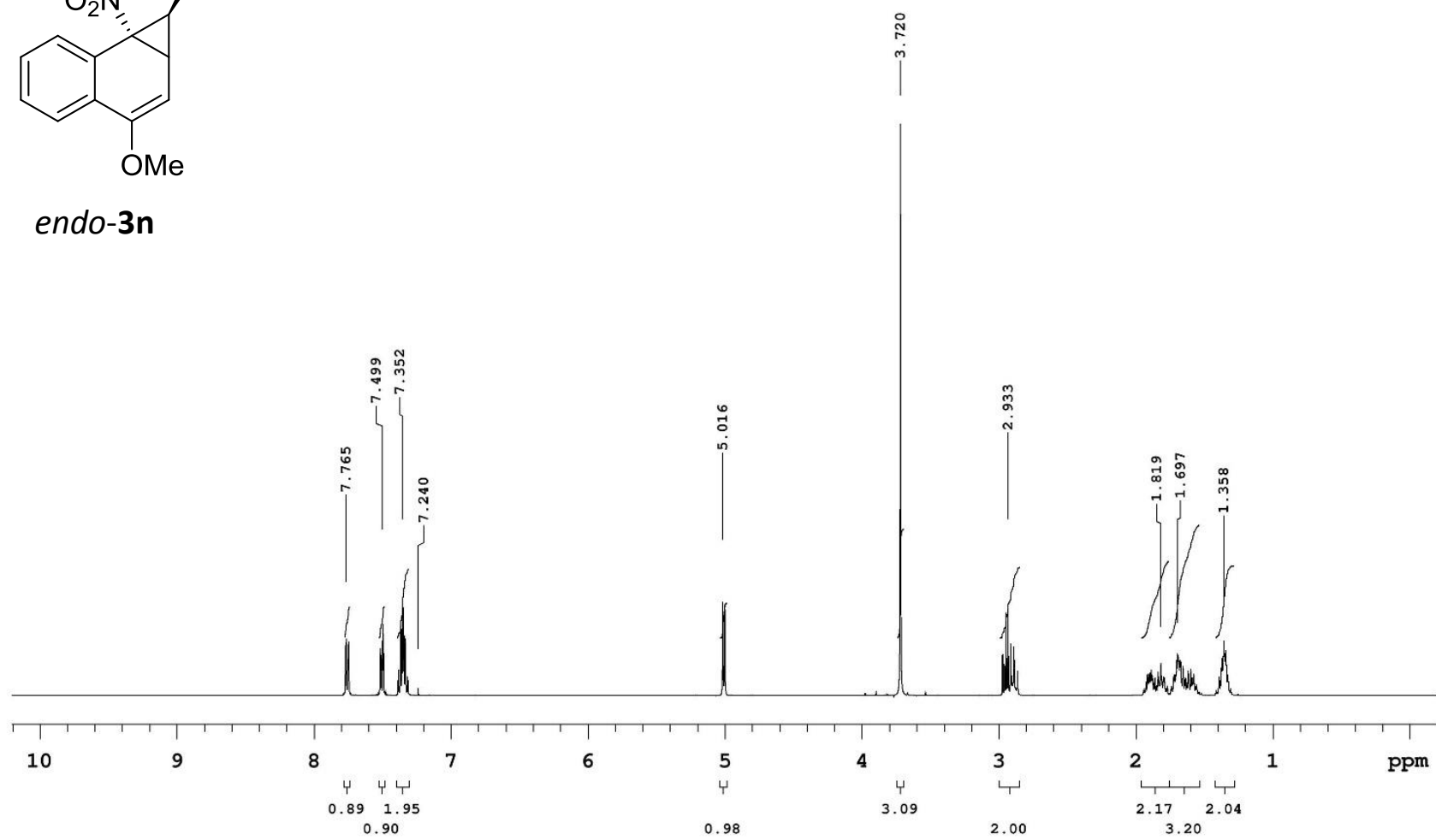

Sample Name: DA-275c

Solvent: cdc13

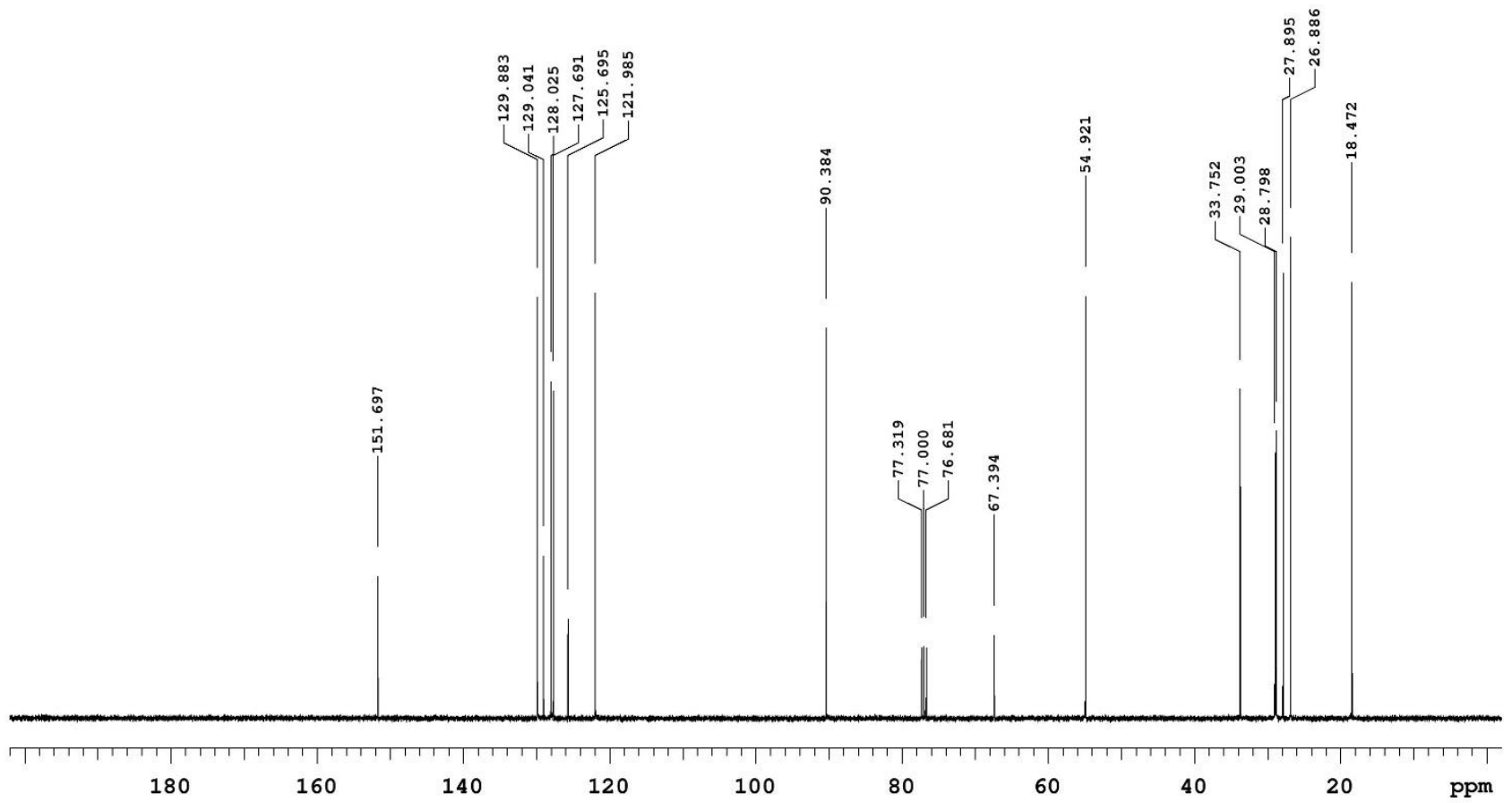


Sample Name: DA-275b

Solvent: cdc13<smiles>COC1=CC2[C@H](C3CCC3)[C@@]2([N+](=O)[O-])c2ccccc21</smiles>

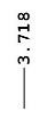

exo-3n

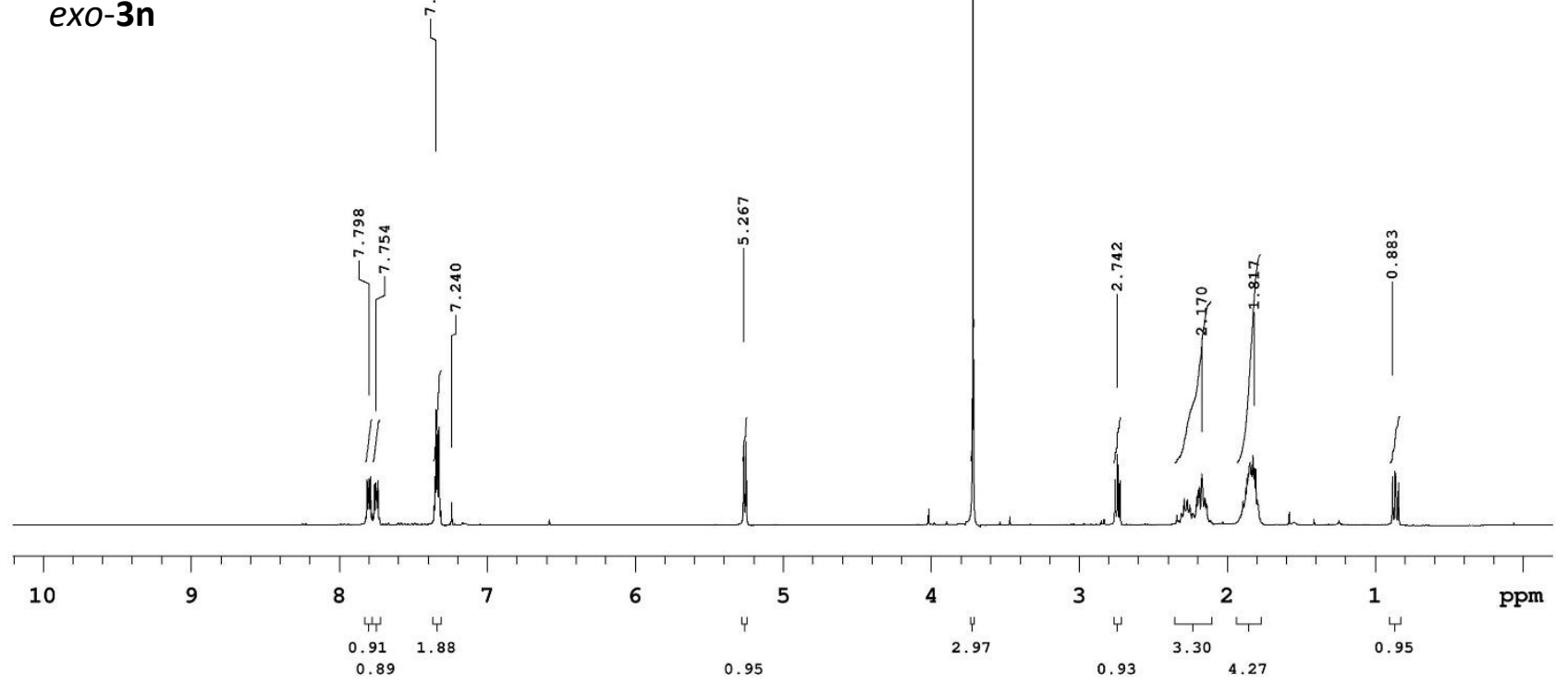

Sample Name: DA-275b

Solvent: cdc13

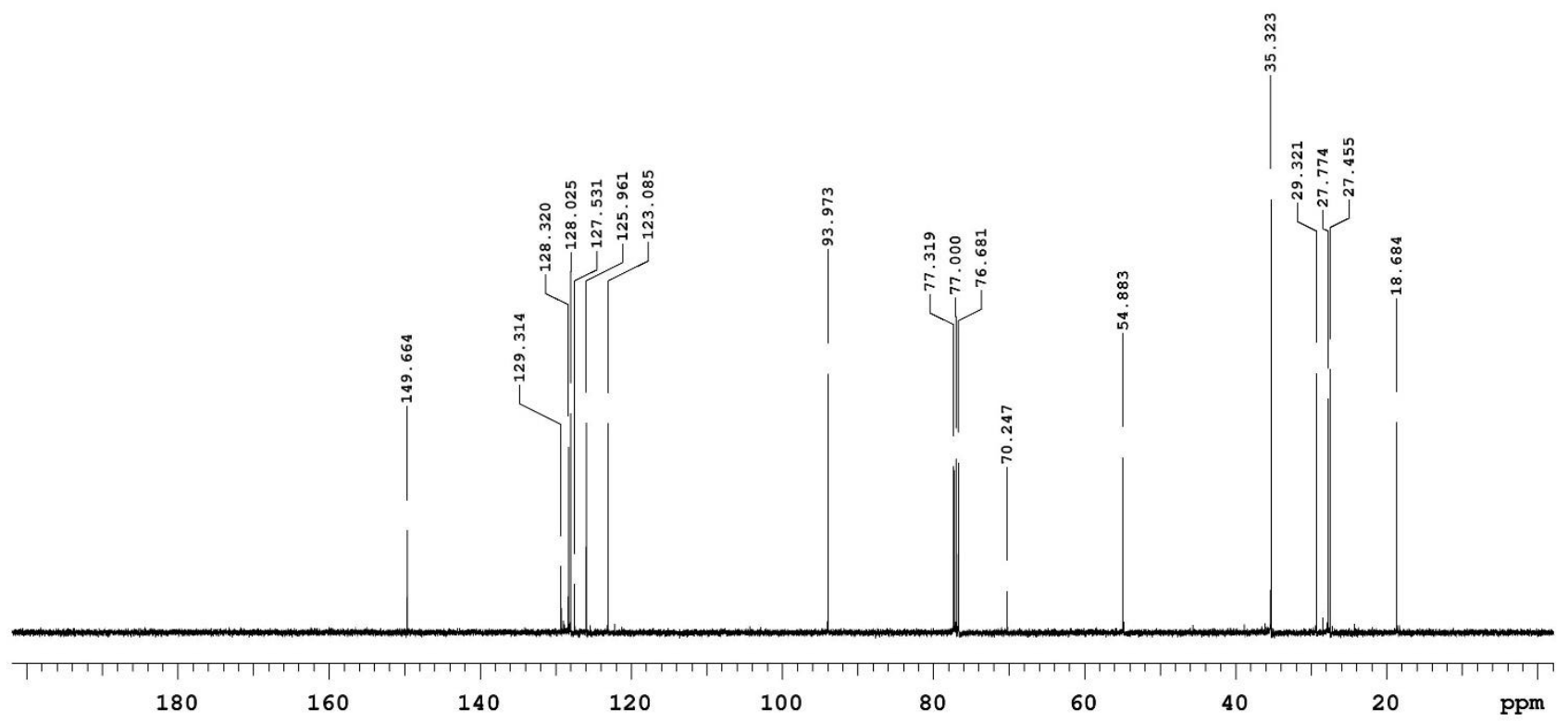




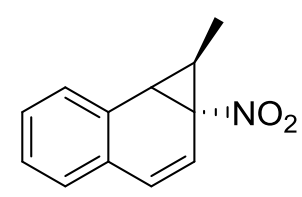

endo-3o

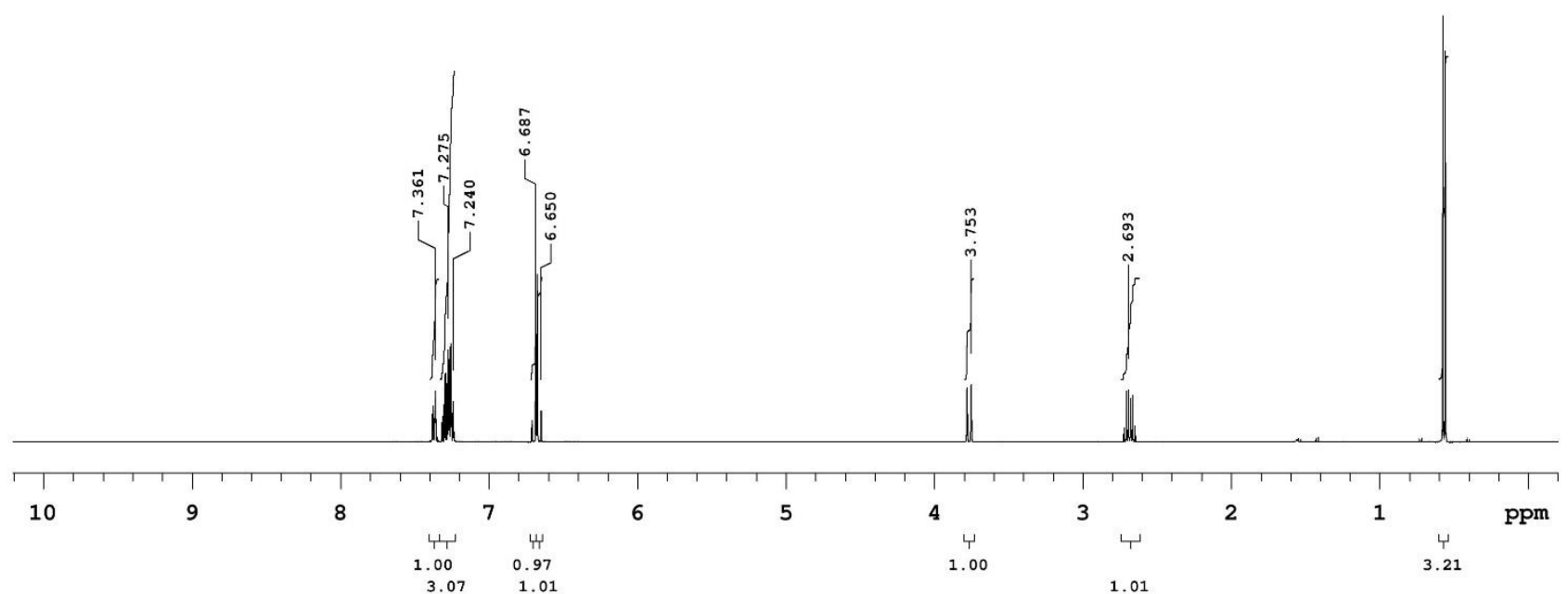

Sample Name: DA-198a b-cryst Solvent: $\operatorname{cdcl} 3$

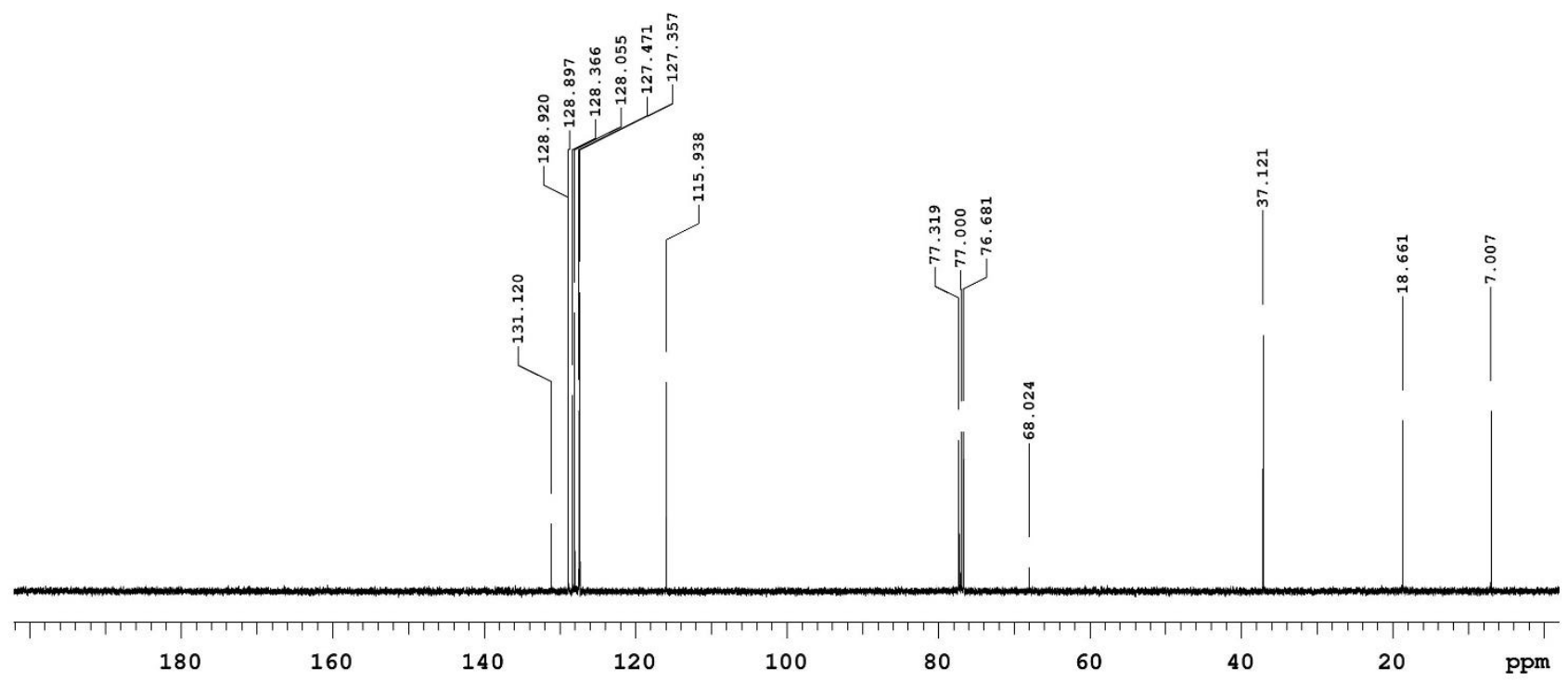




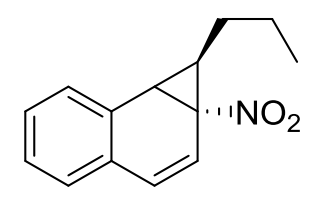

endo-3p

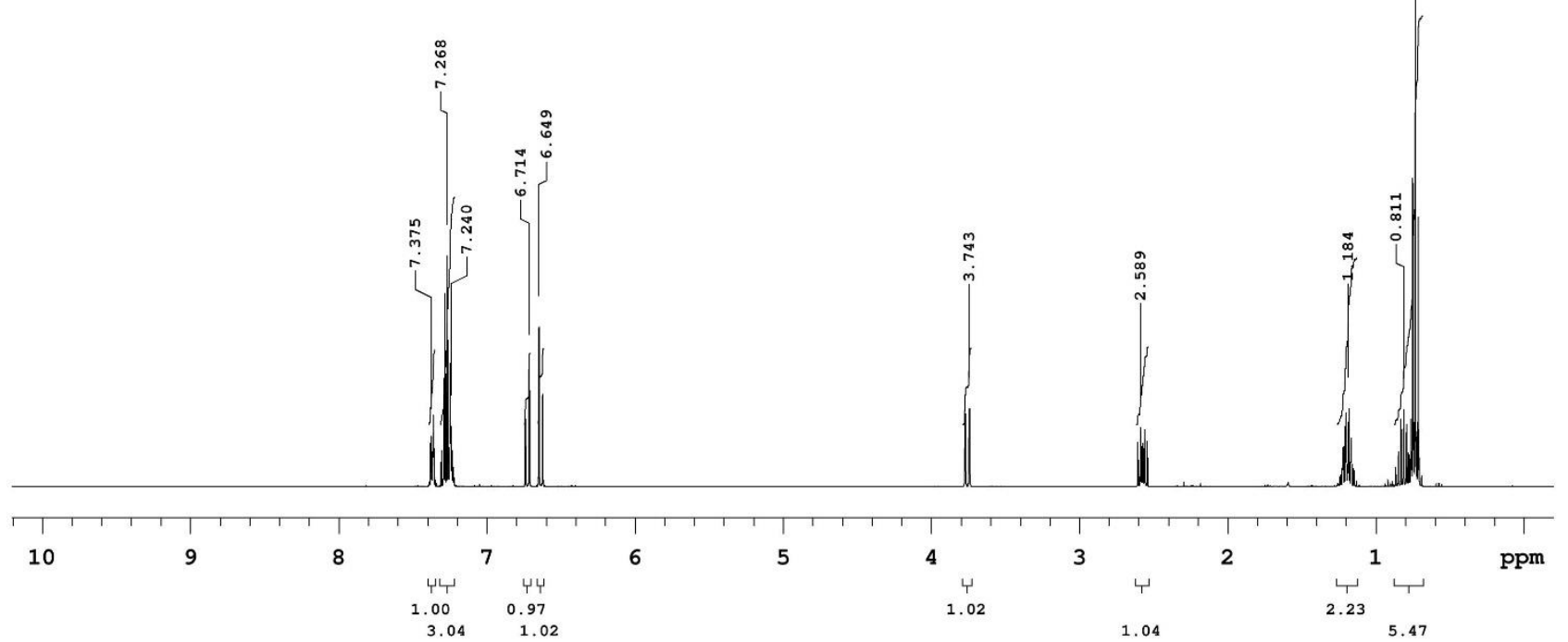

Sample Name: DA-272d

Solvent: cdc13

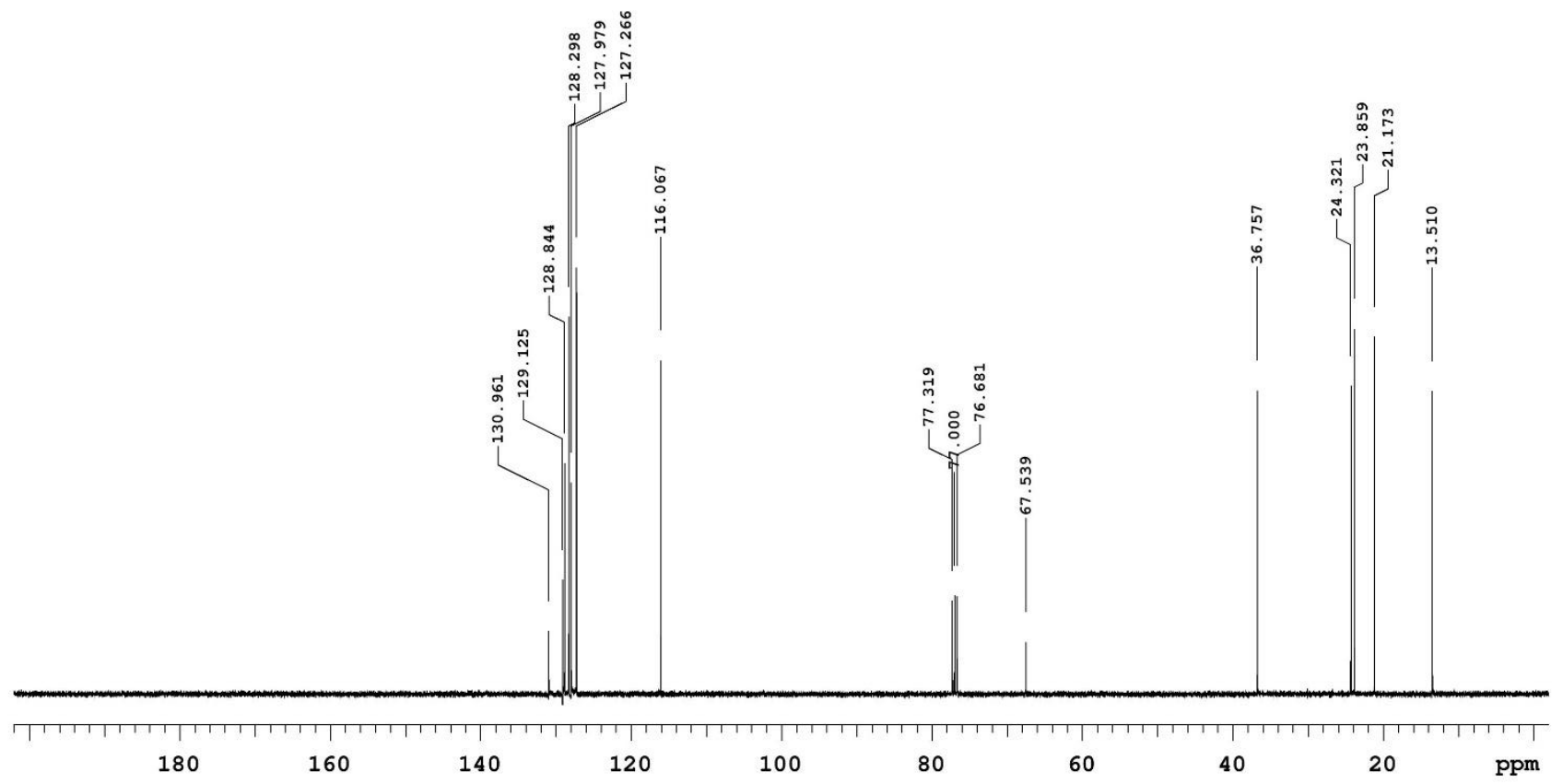



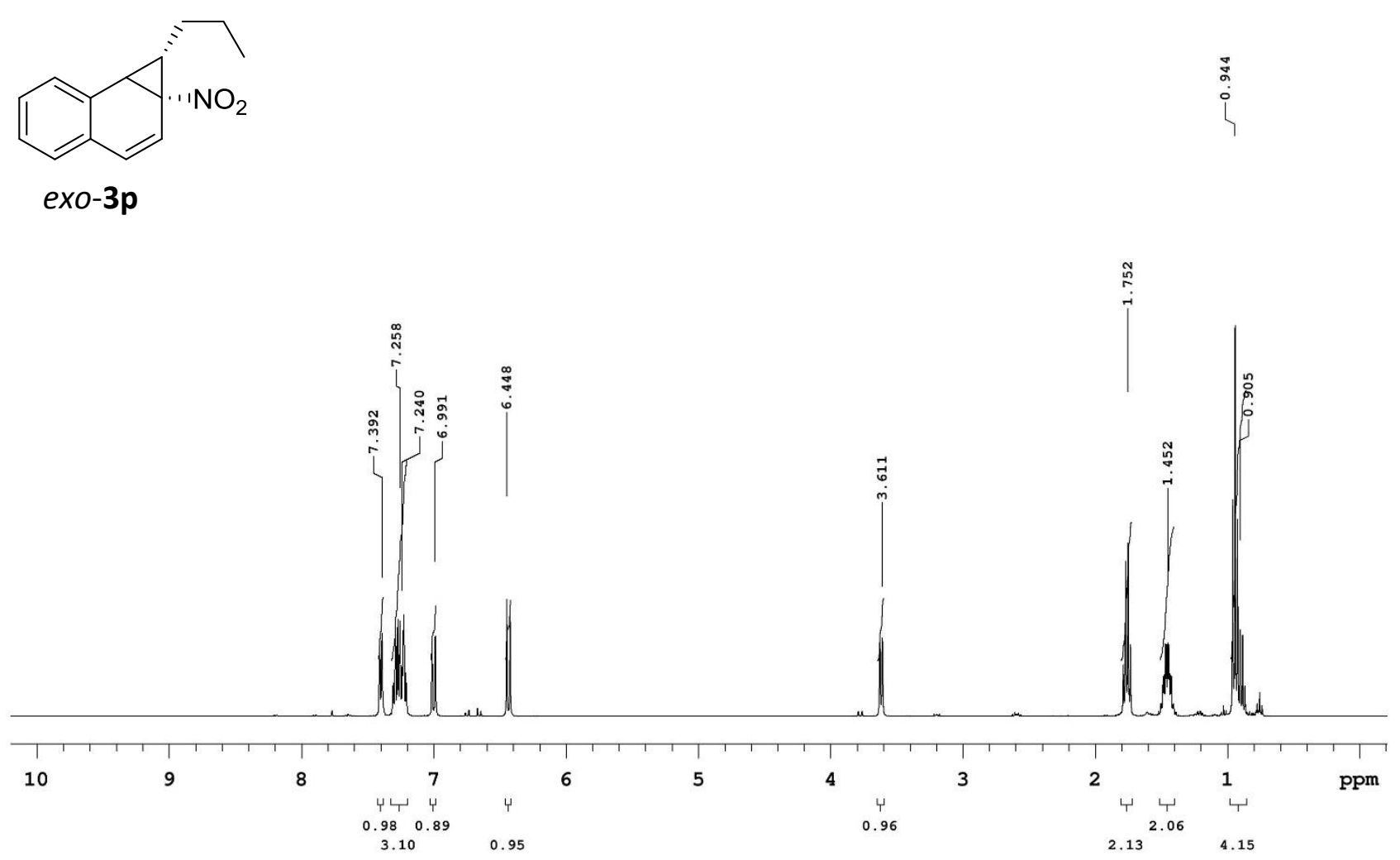

Sample Name: DA-272a Solvent: cdc13

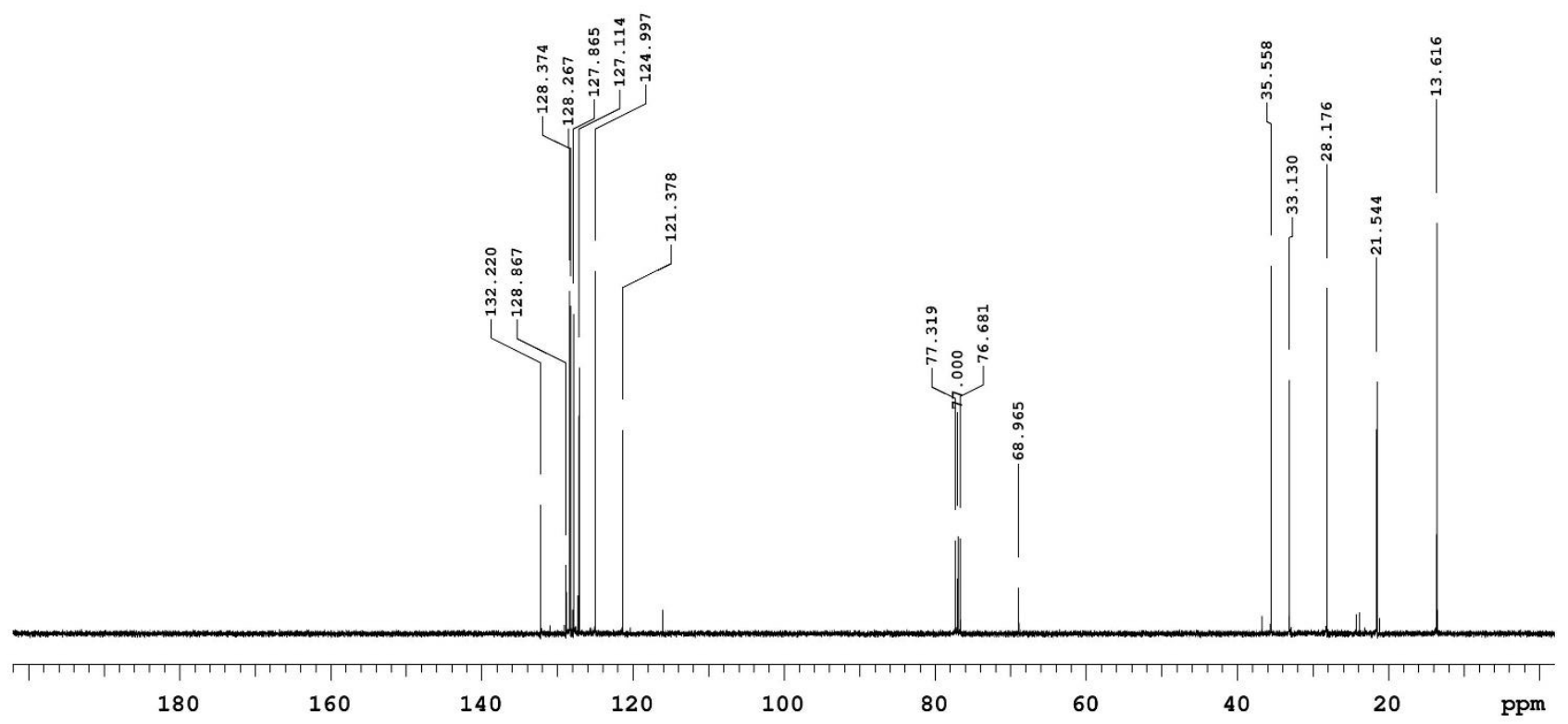


Sample Name: DA-195c

Solvent: cdc13

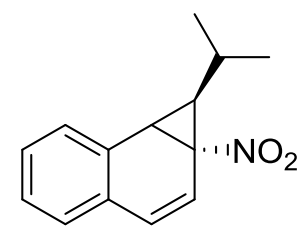

endo-3q

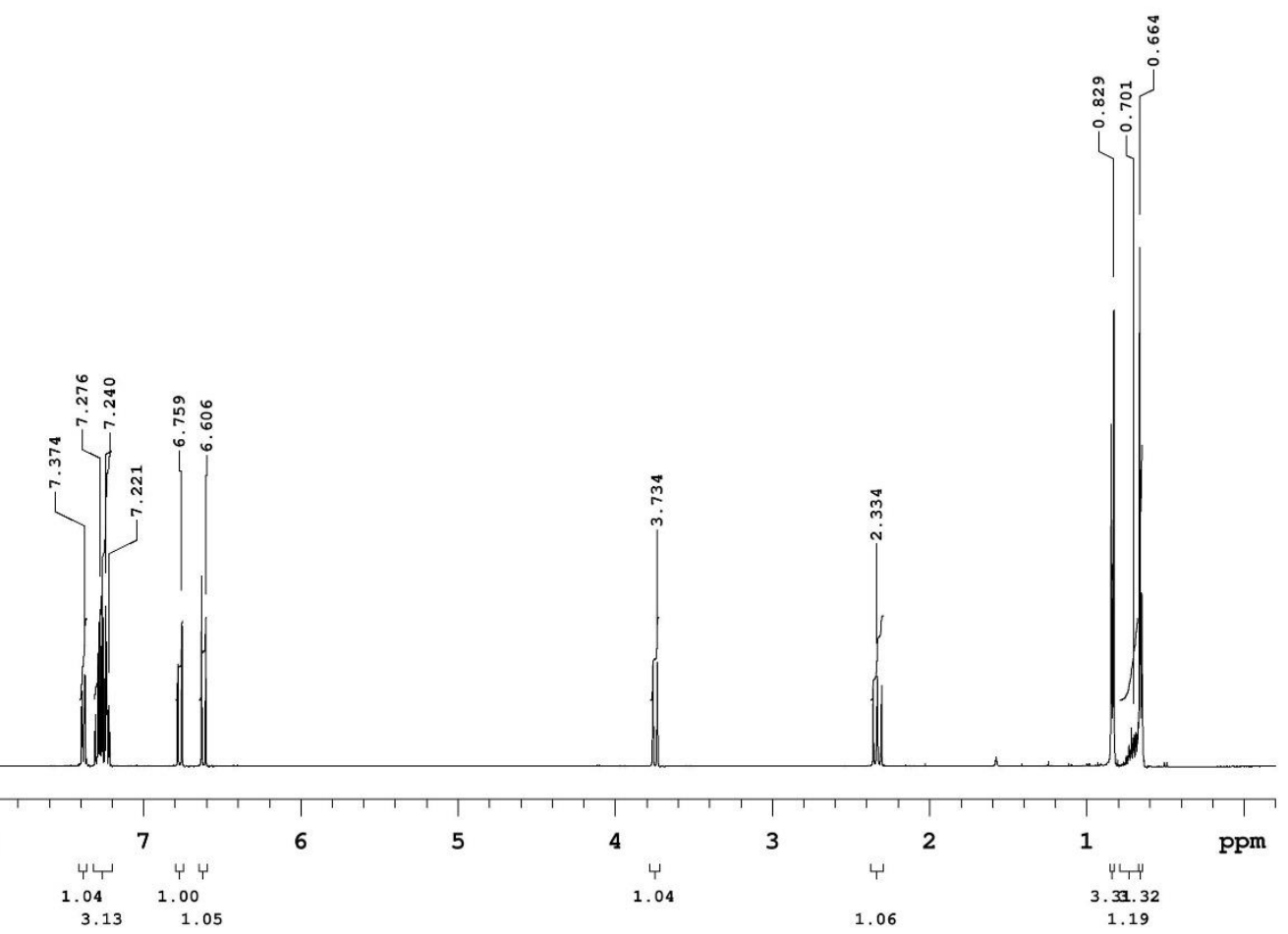

Sample Name: DA-195c

Solvent: $\operatorname{cdcl} 3$

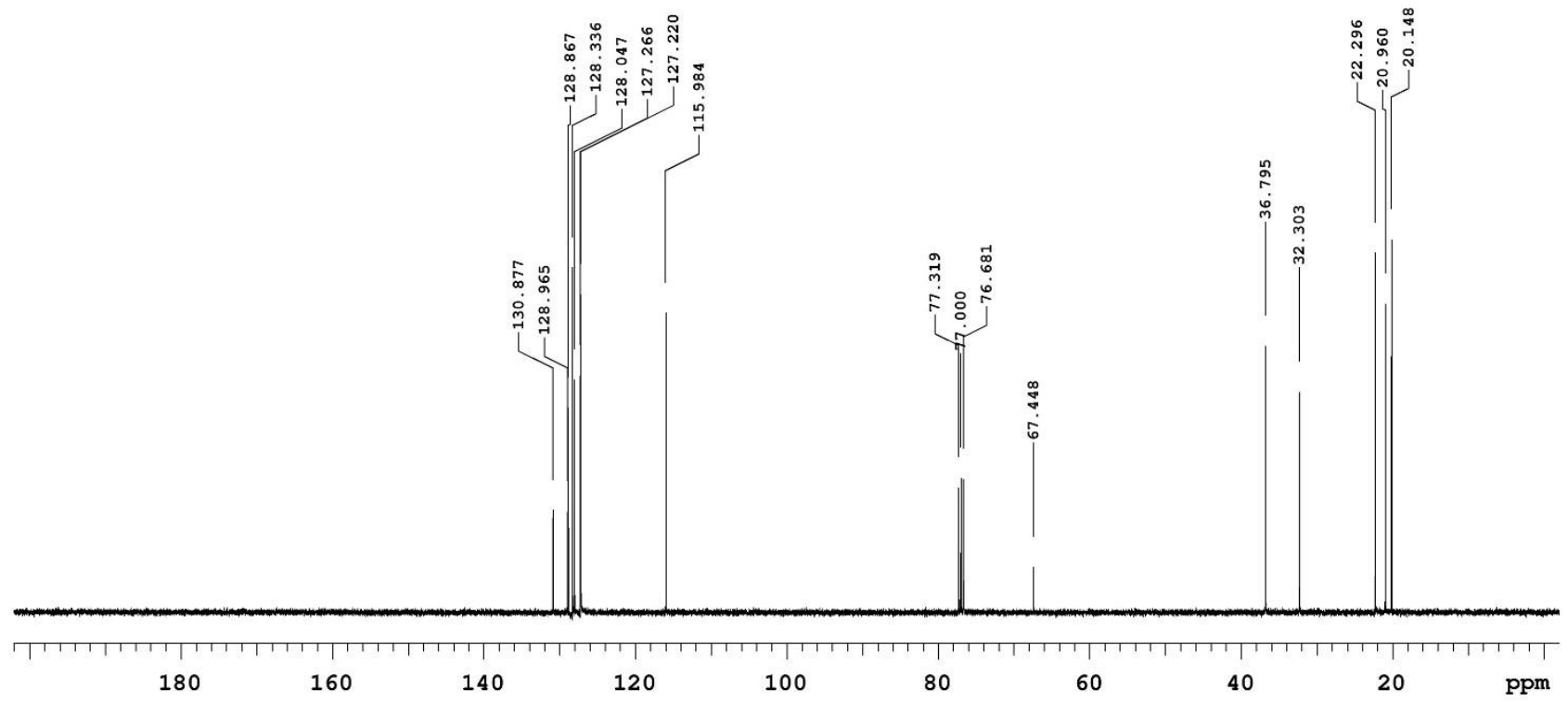


Sample Name: DA-195a

Solvent: cdc13
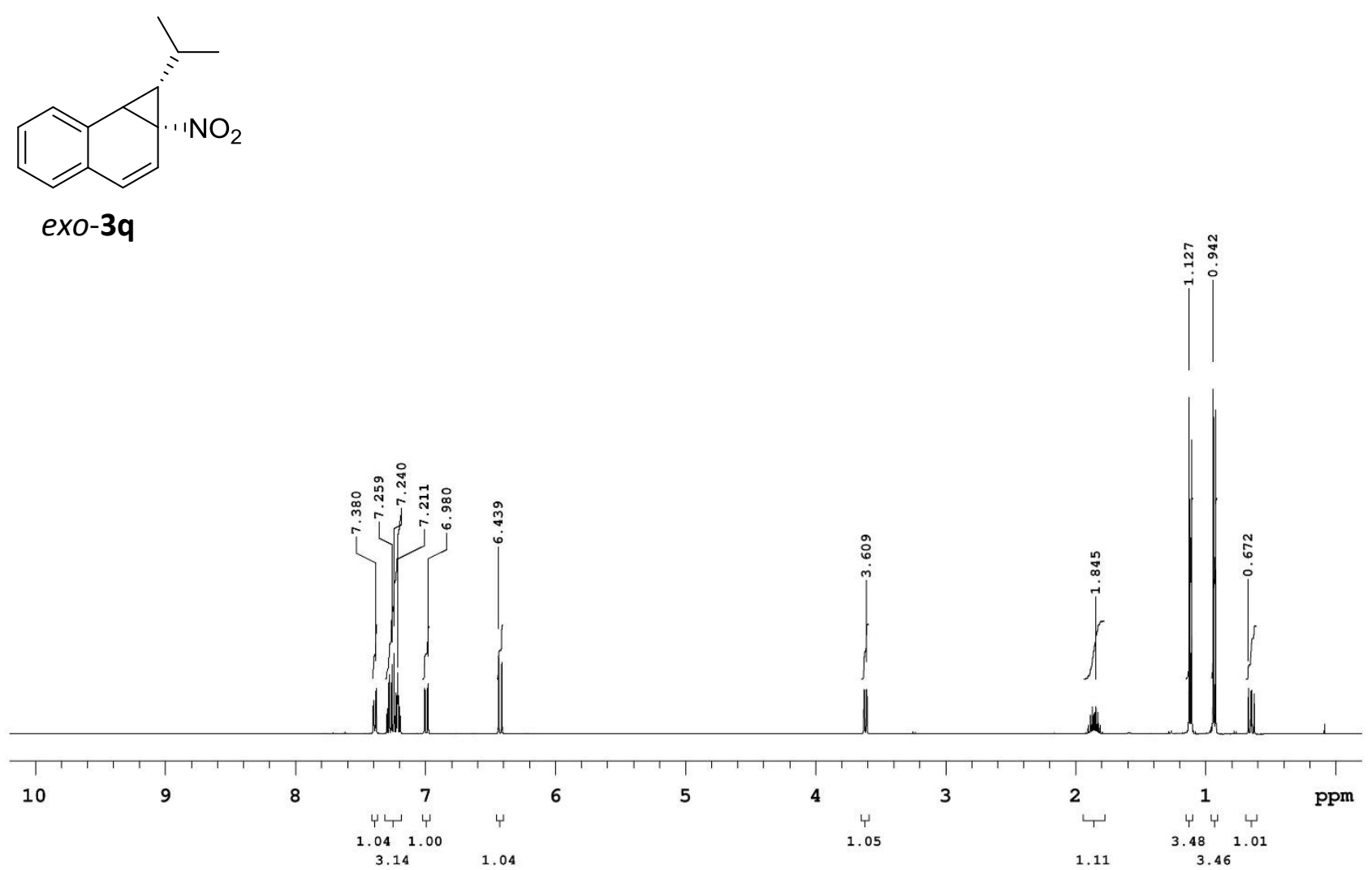

Sample Name: DA-195a

Solvent: cdc13

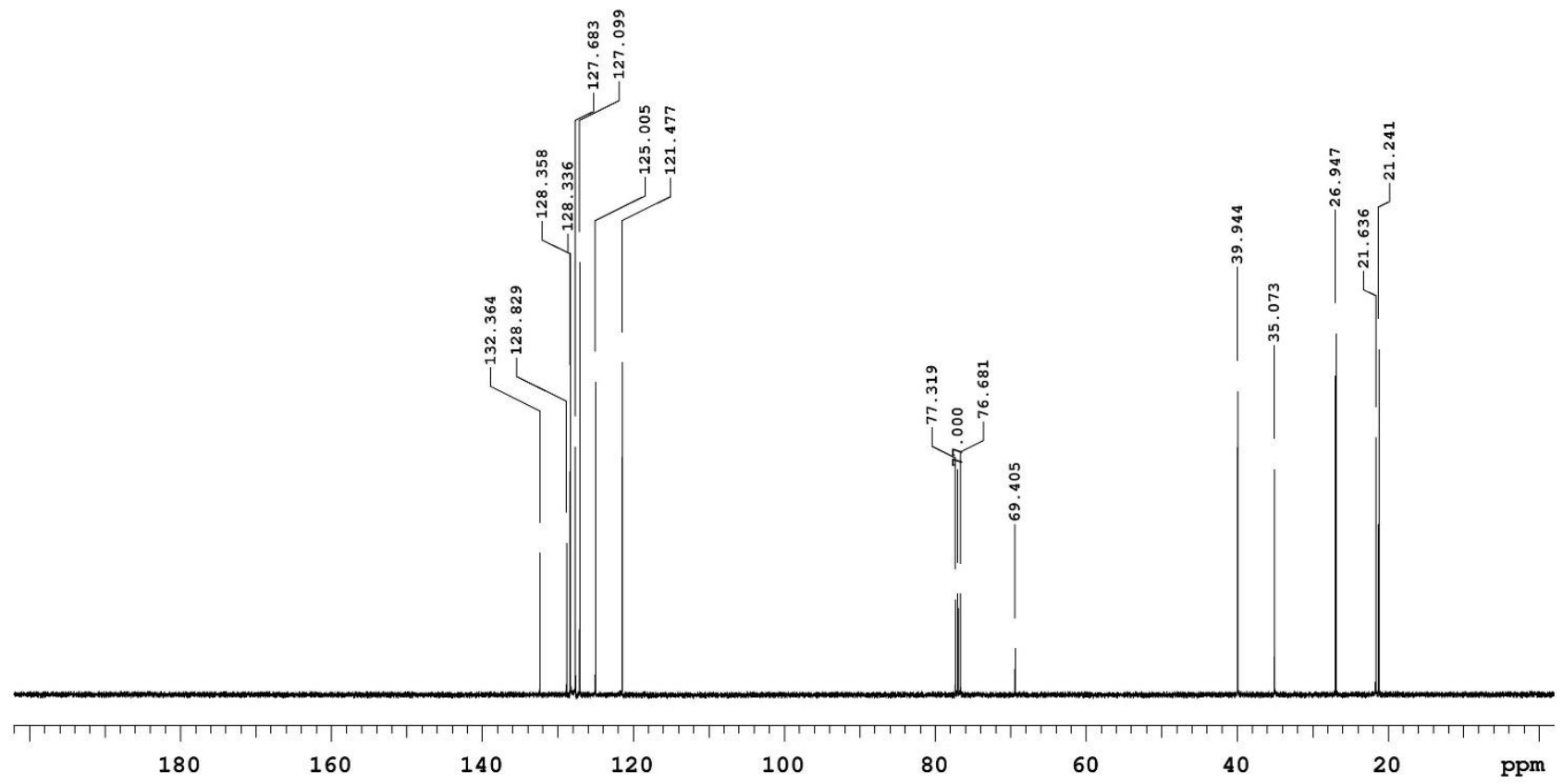


Sample Name: DA-267c

Solvent: cdc13
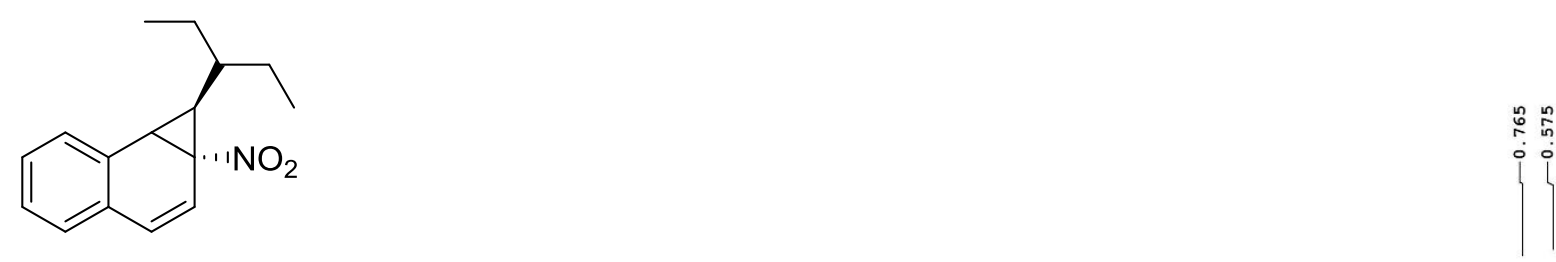

endo-3r

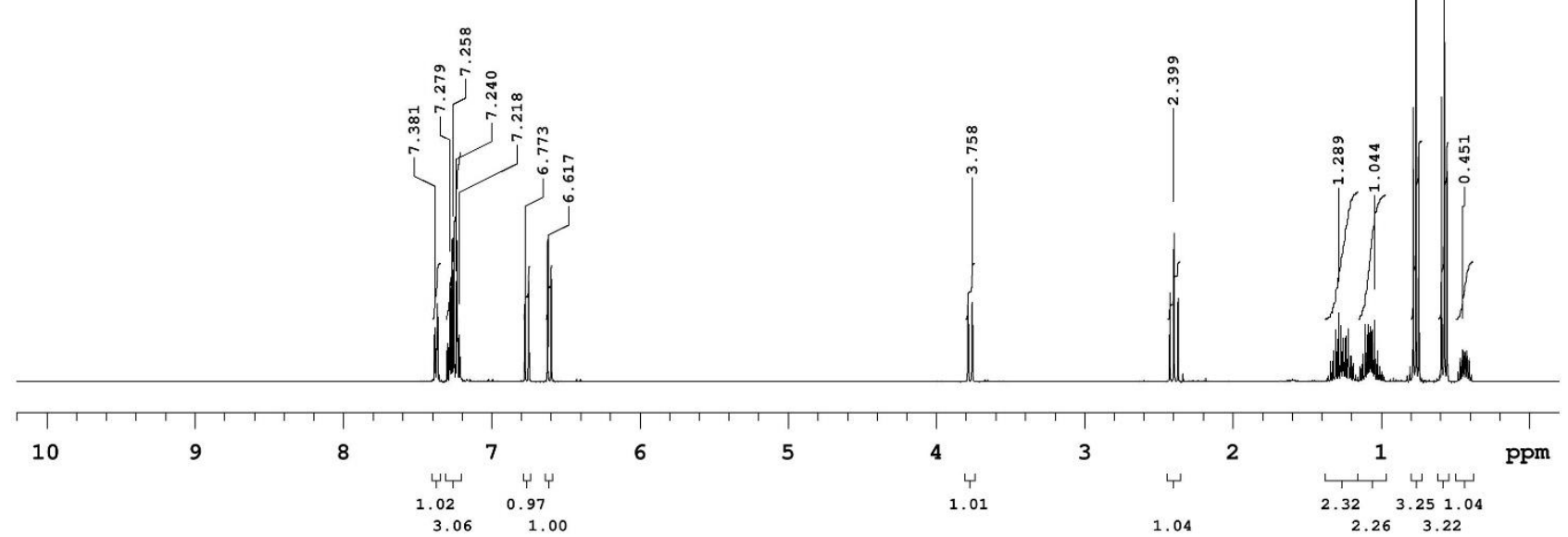

Sample Name: DA-267C

Solvent: cdc13

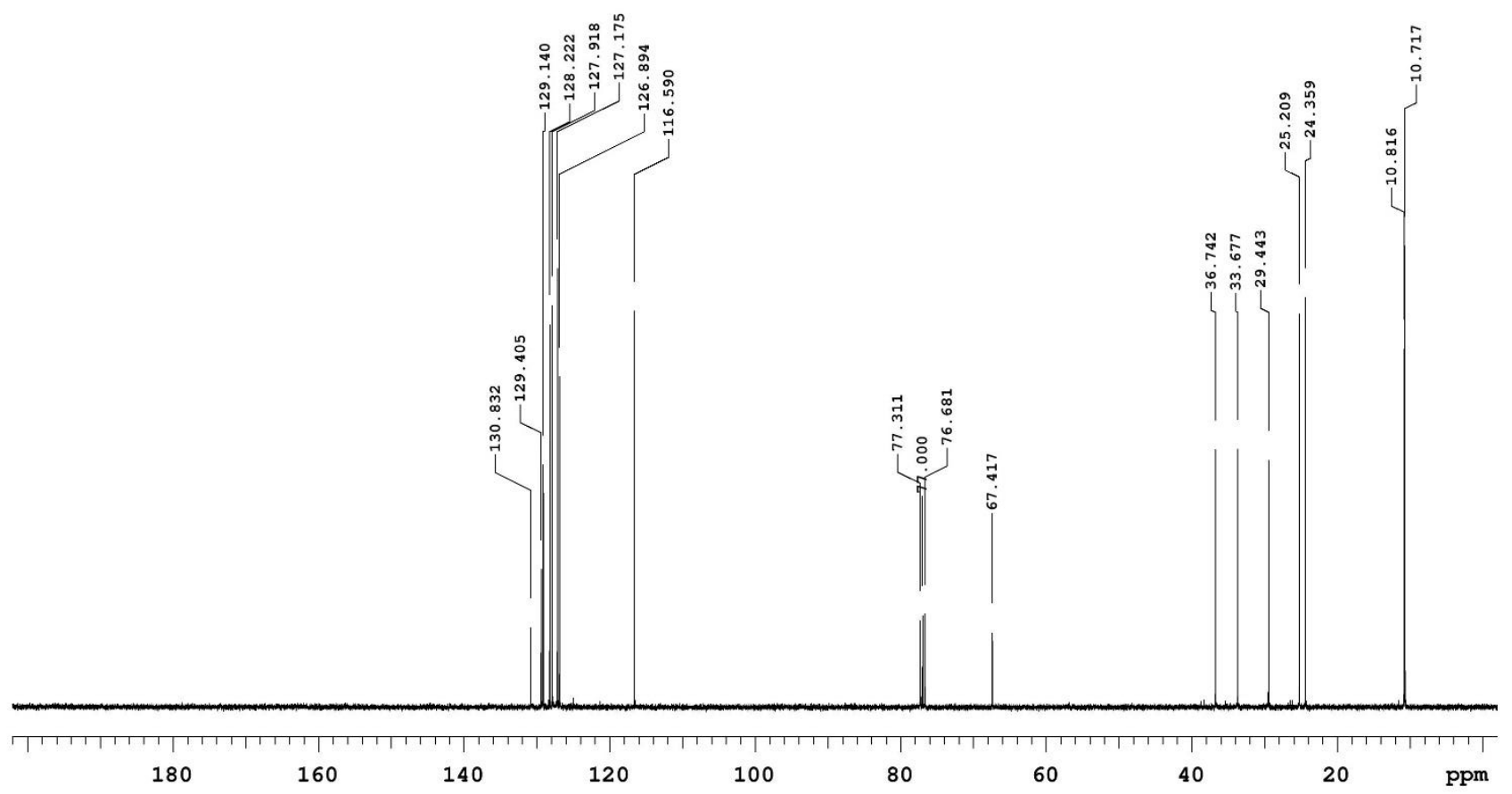


Sample Name: DA-267a

Solvent: cdc13

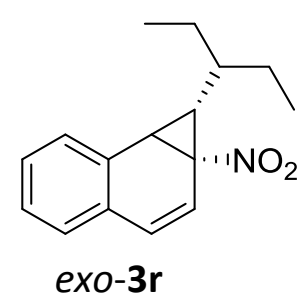

品웅웅
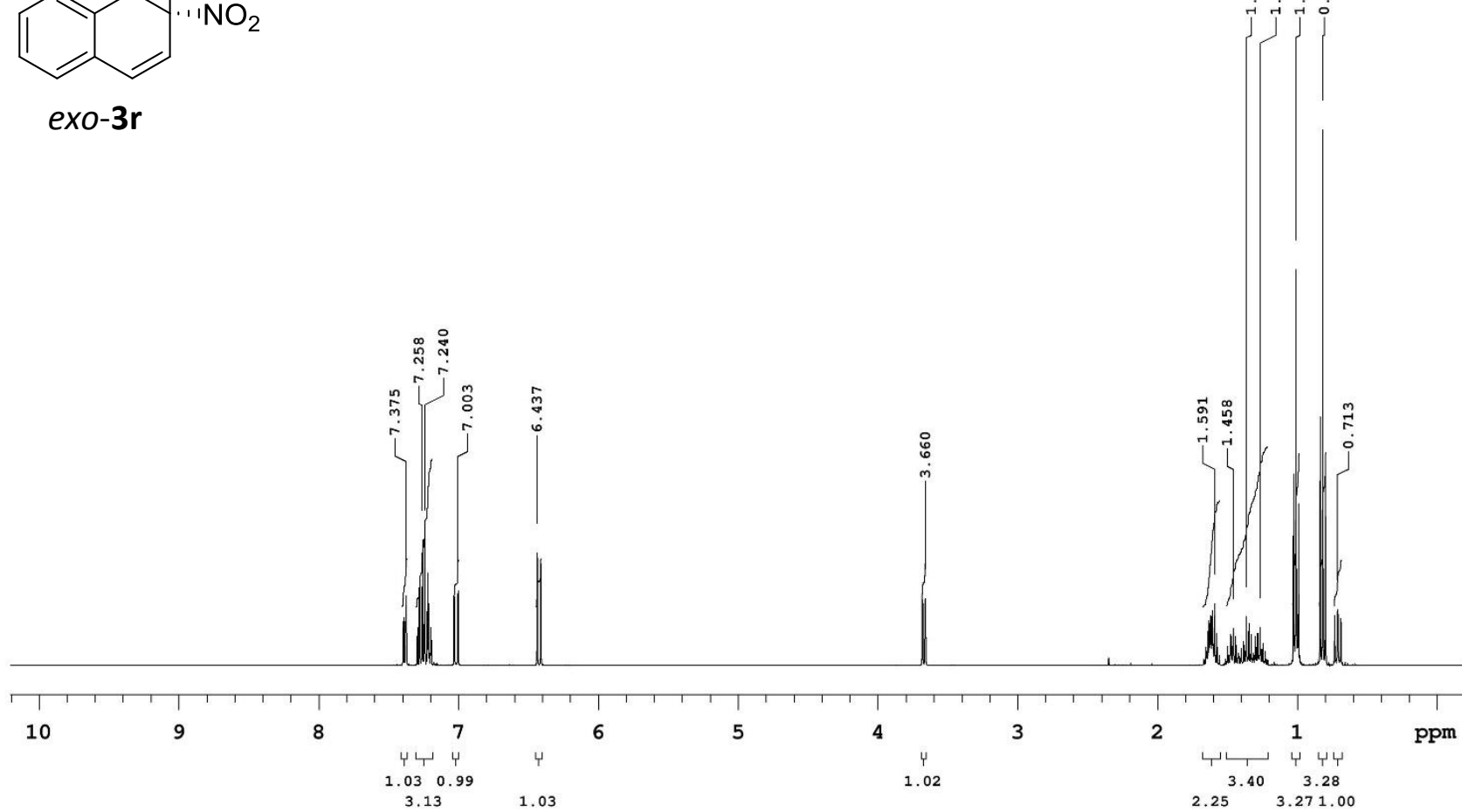

Sample Name: DA-267a

Solvent: $\operatorname{cdcl} 3$

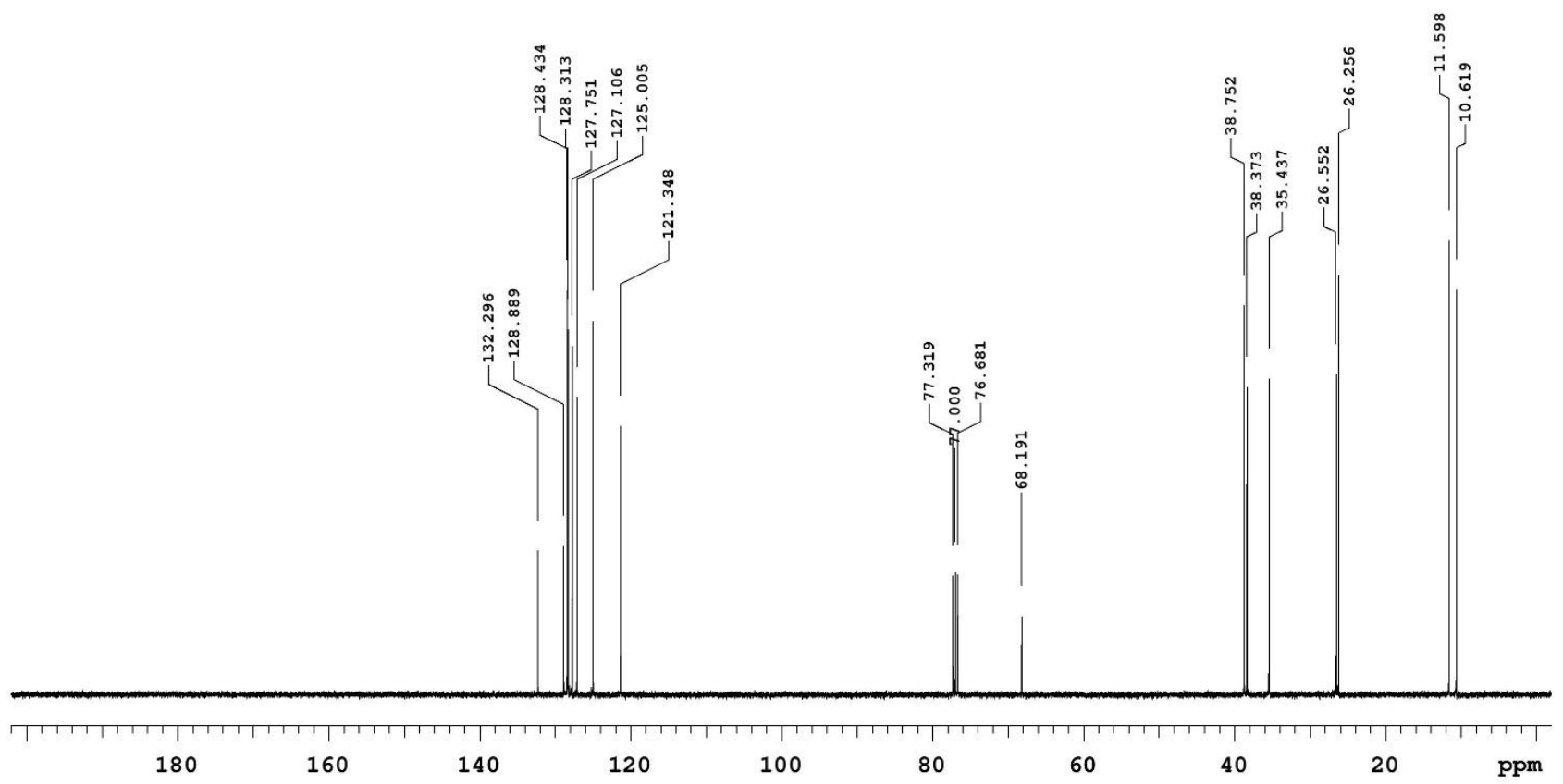


Sample Name: DA-207C

Solvent: cdc13

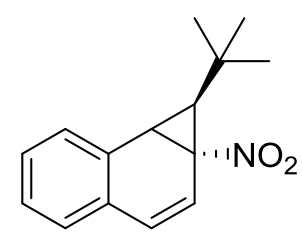

endo-3s

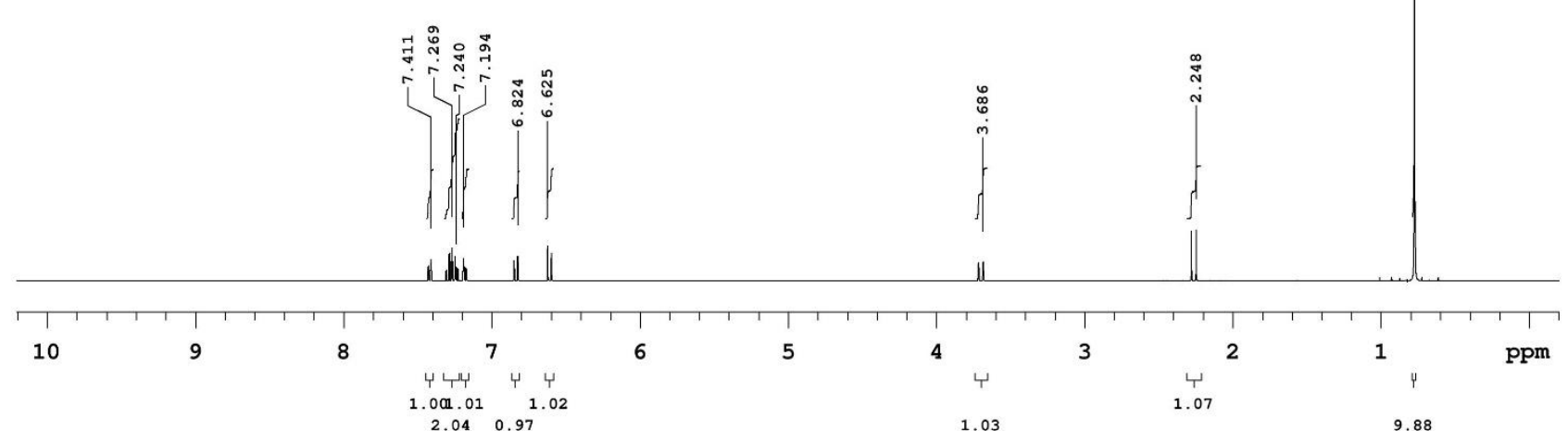

Sample Name: DA-207c

Solvent: cdc13

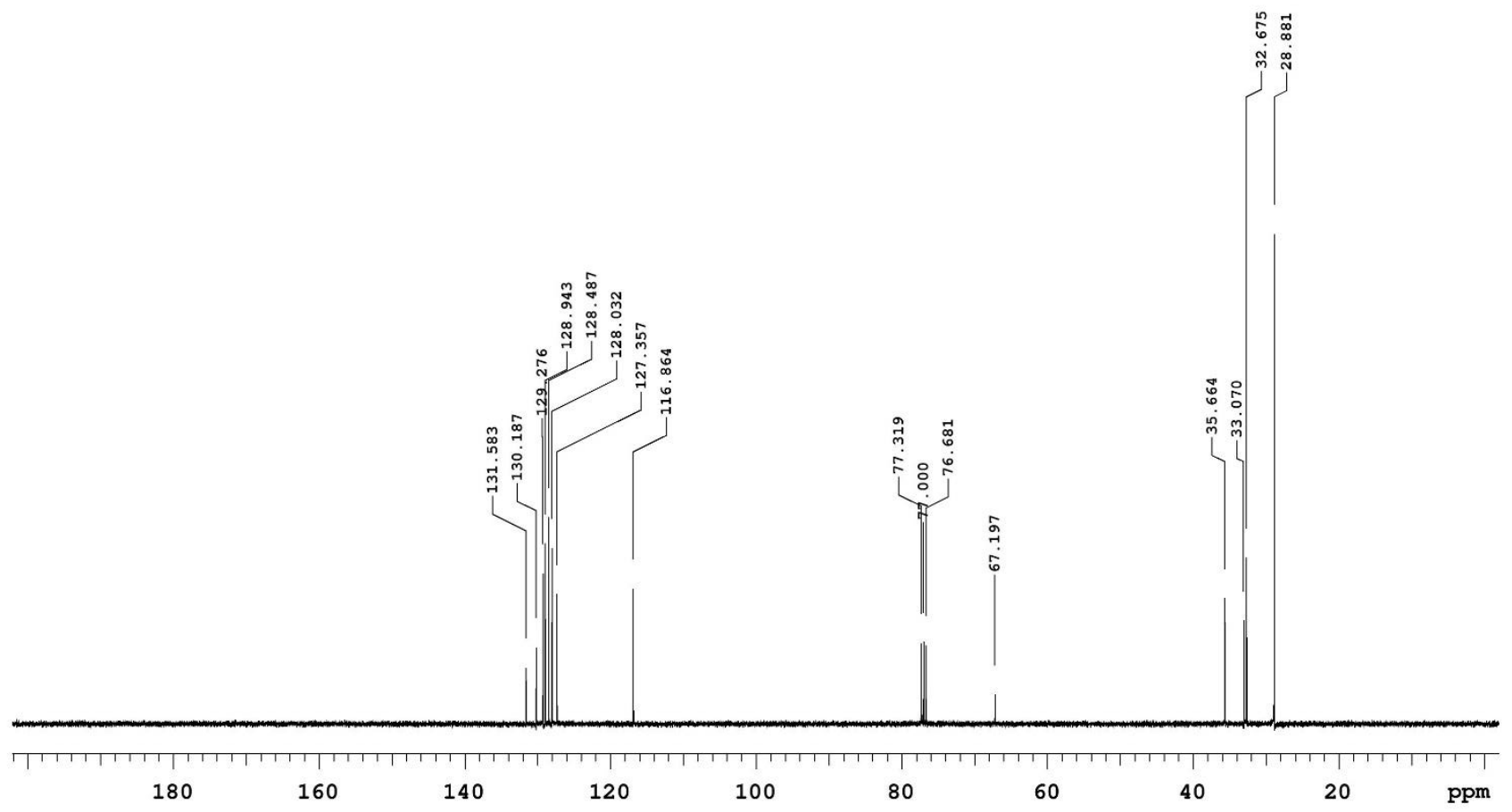


Sample Name: DA-207a

Solvent: cdc13
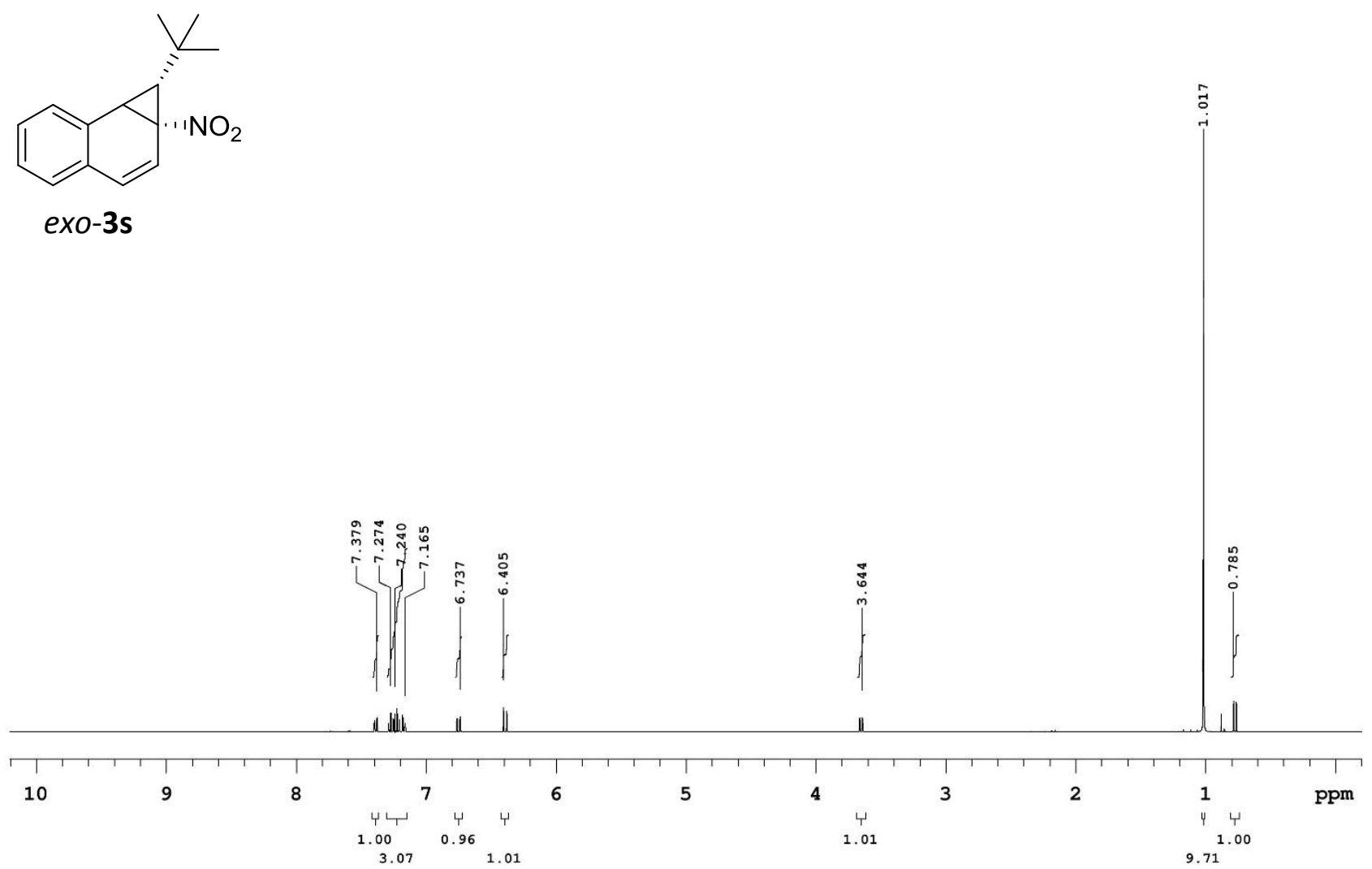

Sample Name: DA-207a

Solvent: cdc13

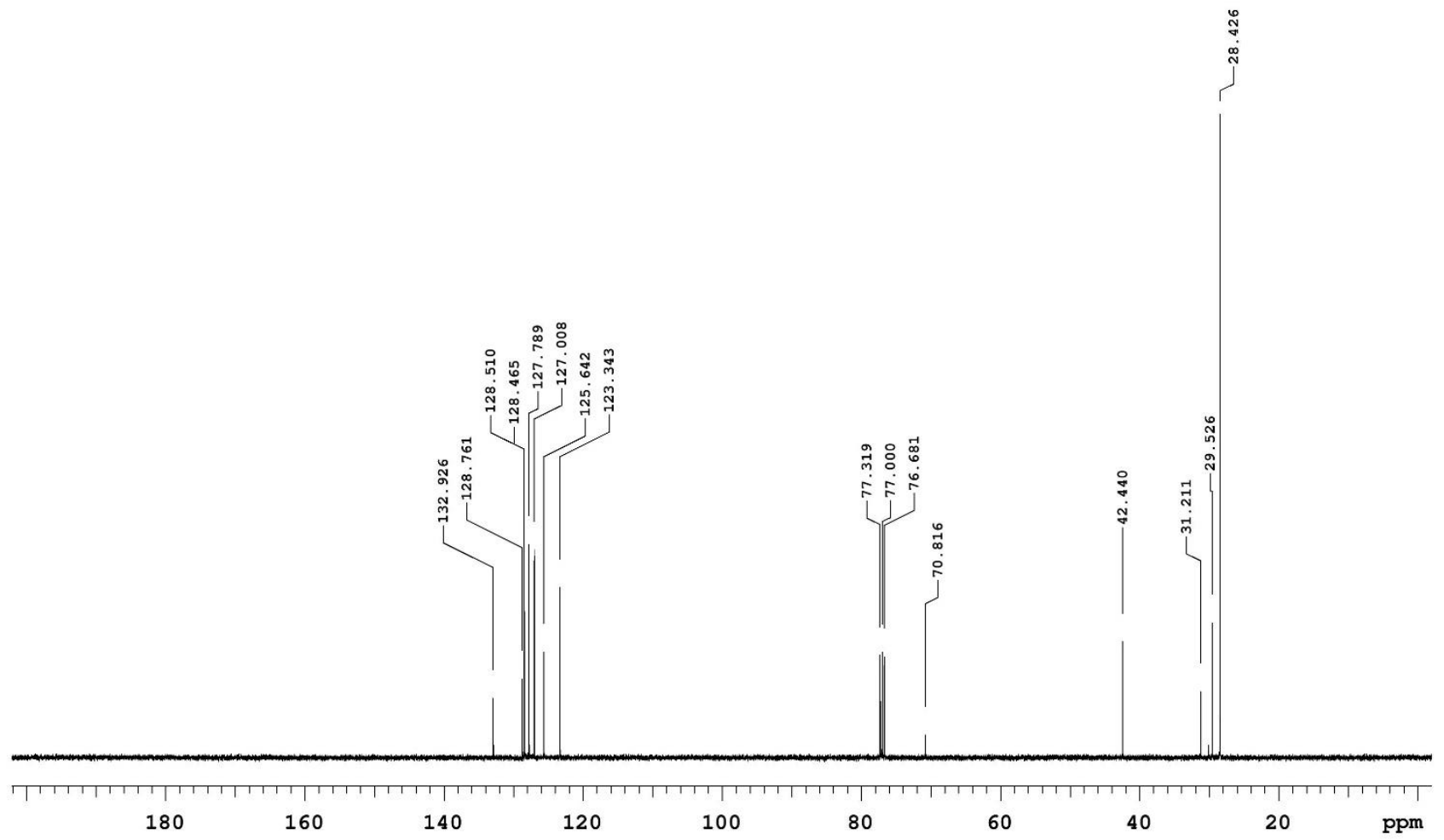


Sample Name: DA-268b

Solvent: cdc13

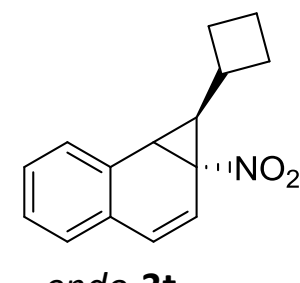

endo-3t
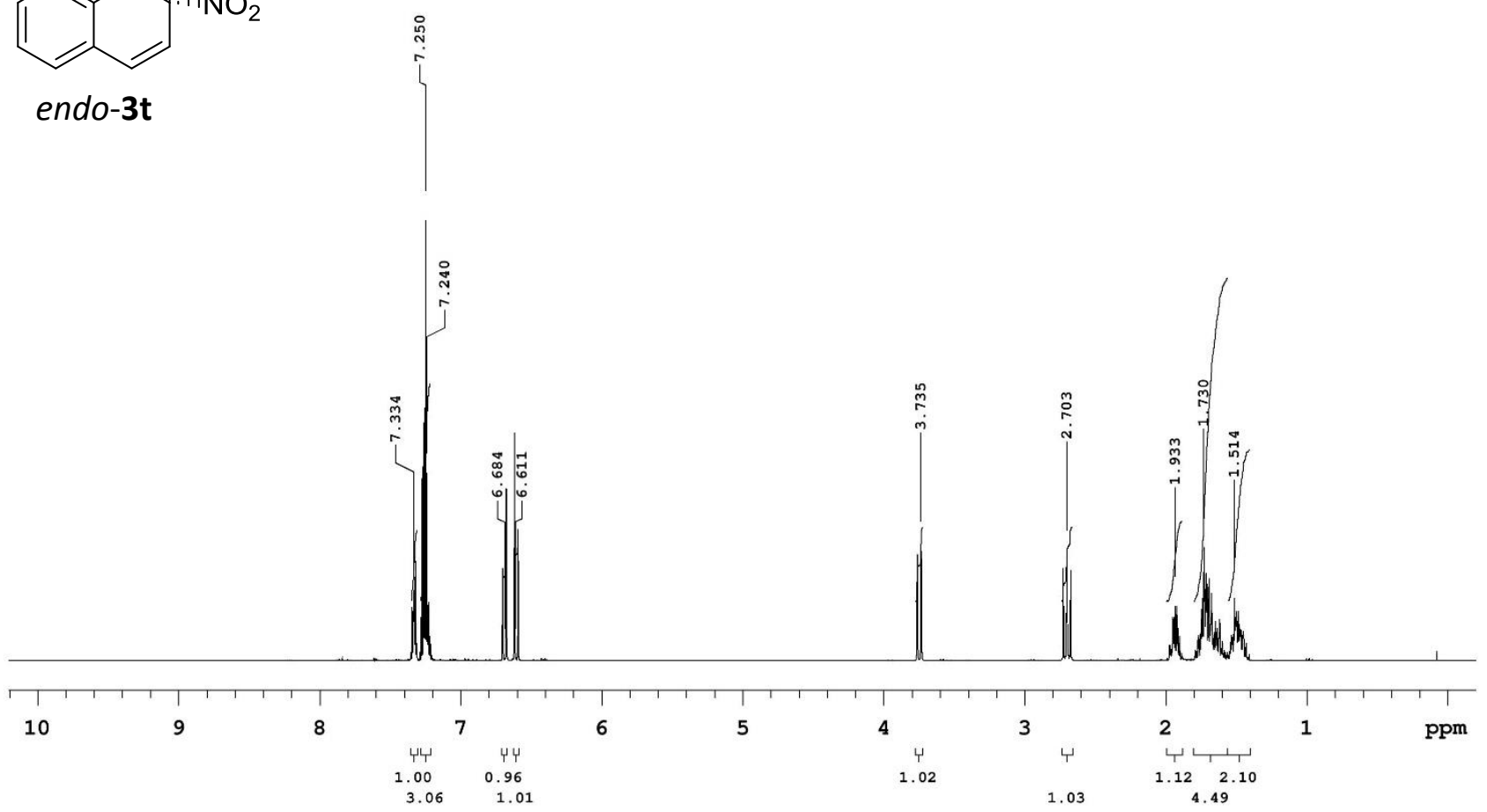

Sample Name: DA-268b

Solvent: cdc13

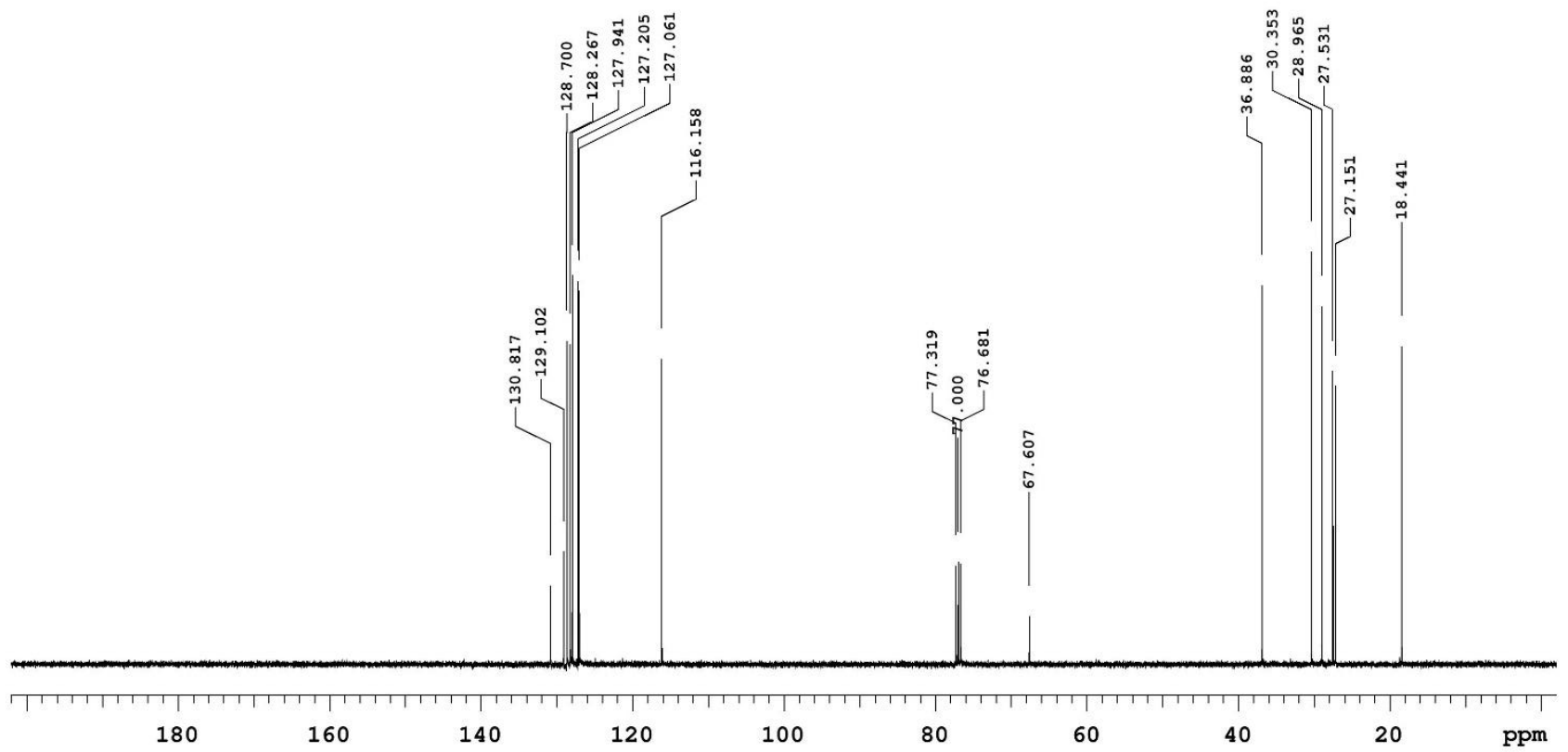


Sample Name: DA-273c

Solvent: $\operatorname{cdcl3}$

(N)

endo-3u

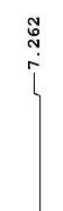

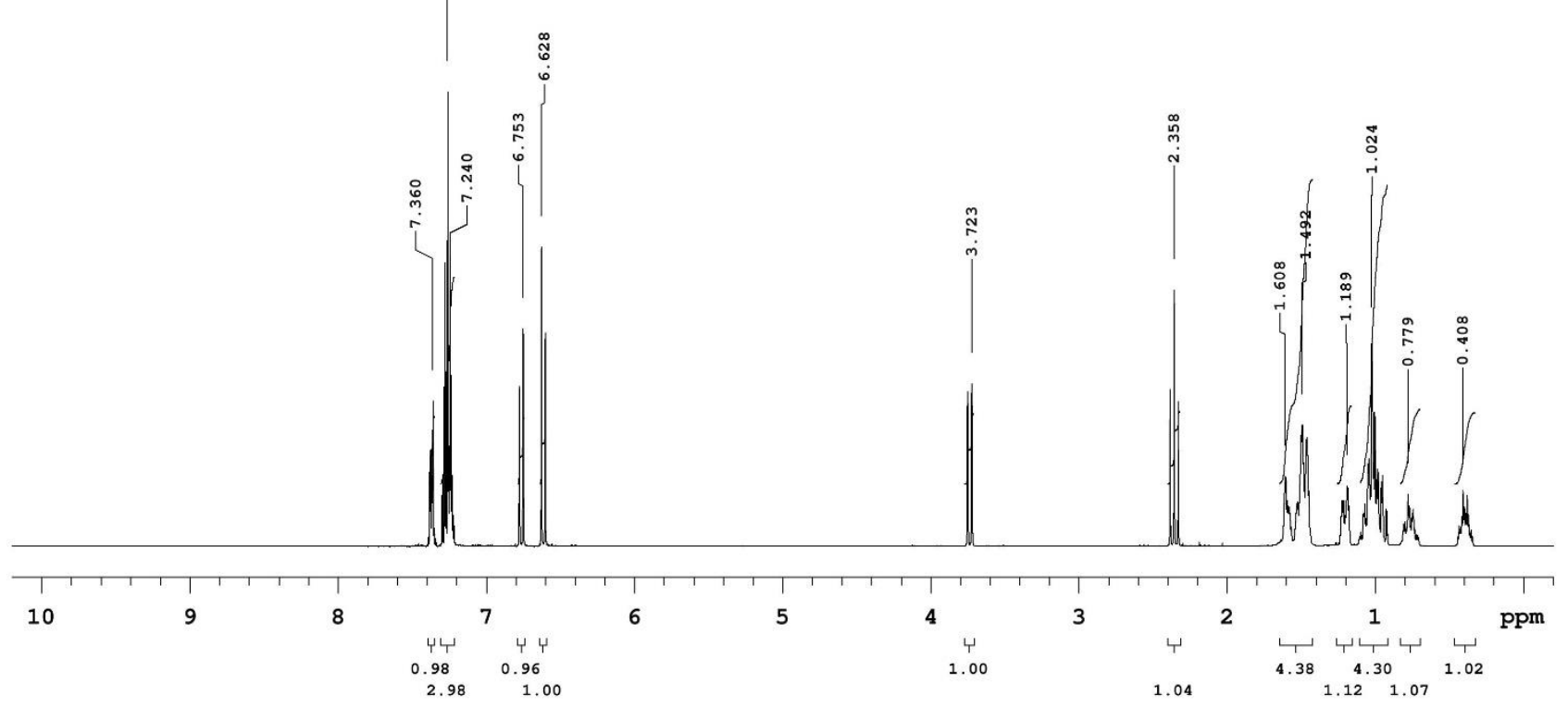

Sample Name: DA-273C

Solvent: cdc13

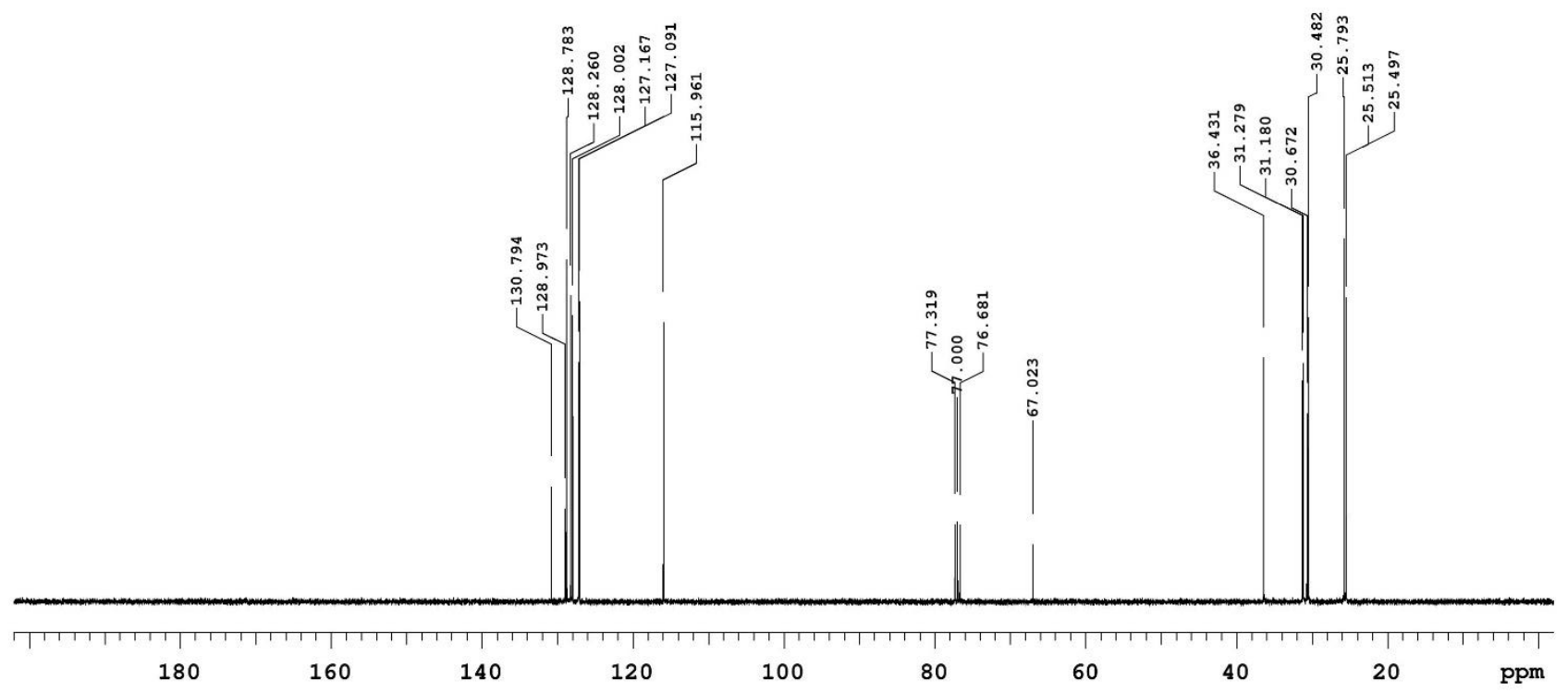


Sample Name: DA-273a

Solvent: $\operatorname{cdcl} 3$

exo-3u

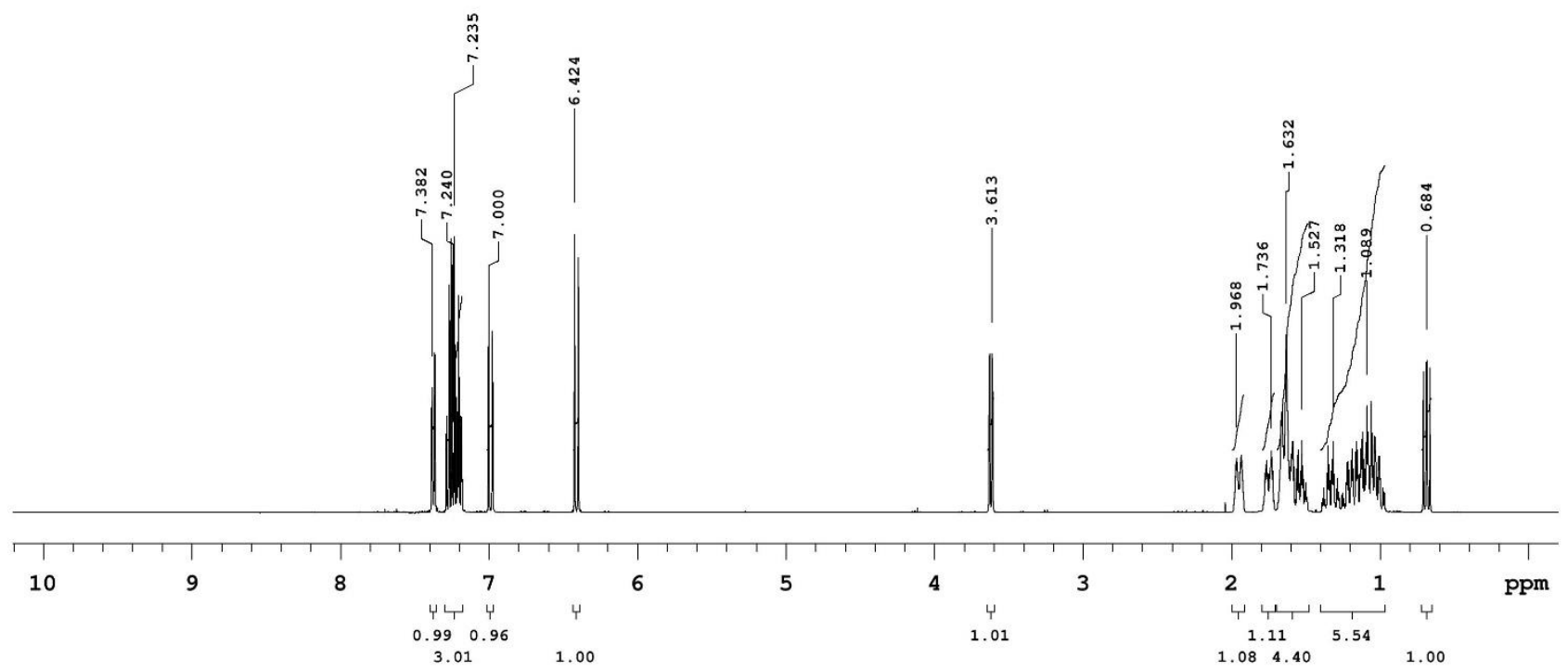

Sample Name: DA-273a

Solvent: cdc13

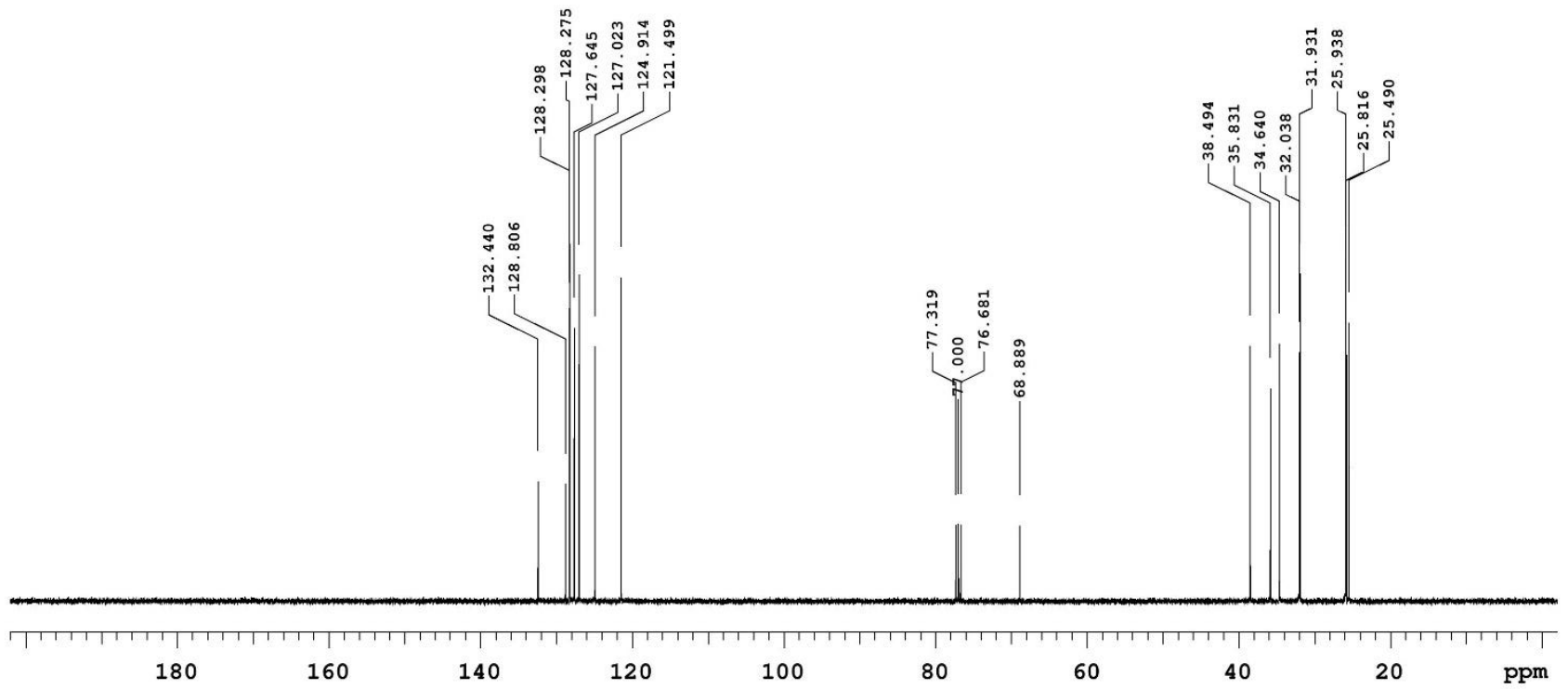




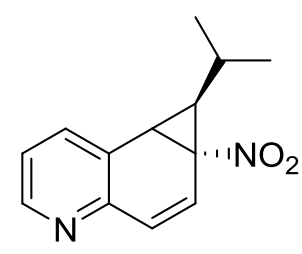

endo-3v

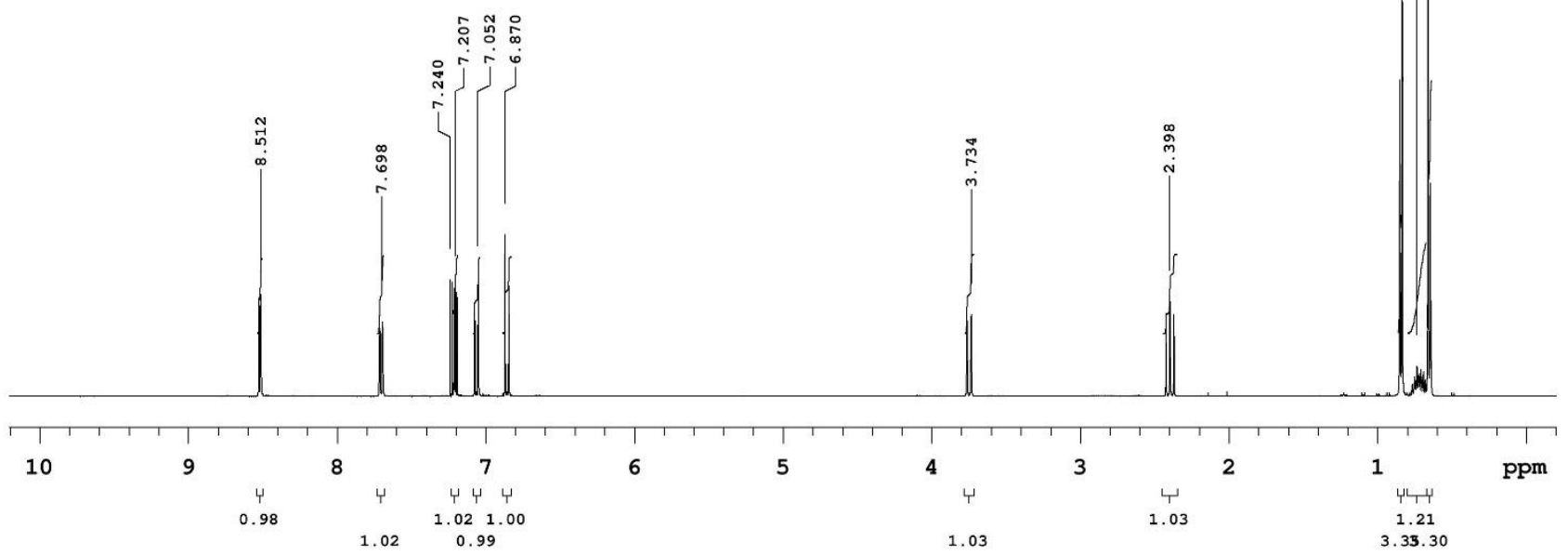

Sample Name: DA-190bc_bb-cryst Solvent: cdc13

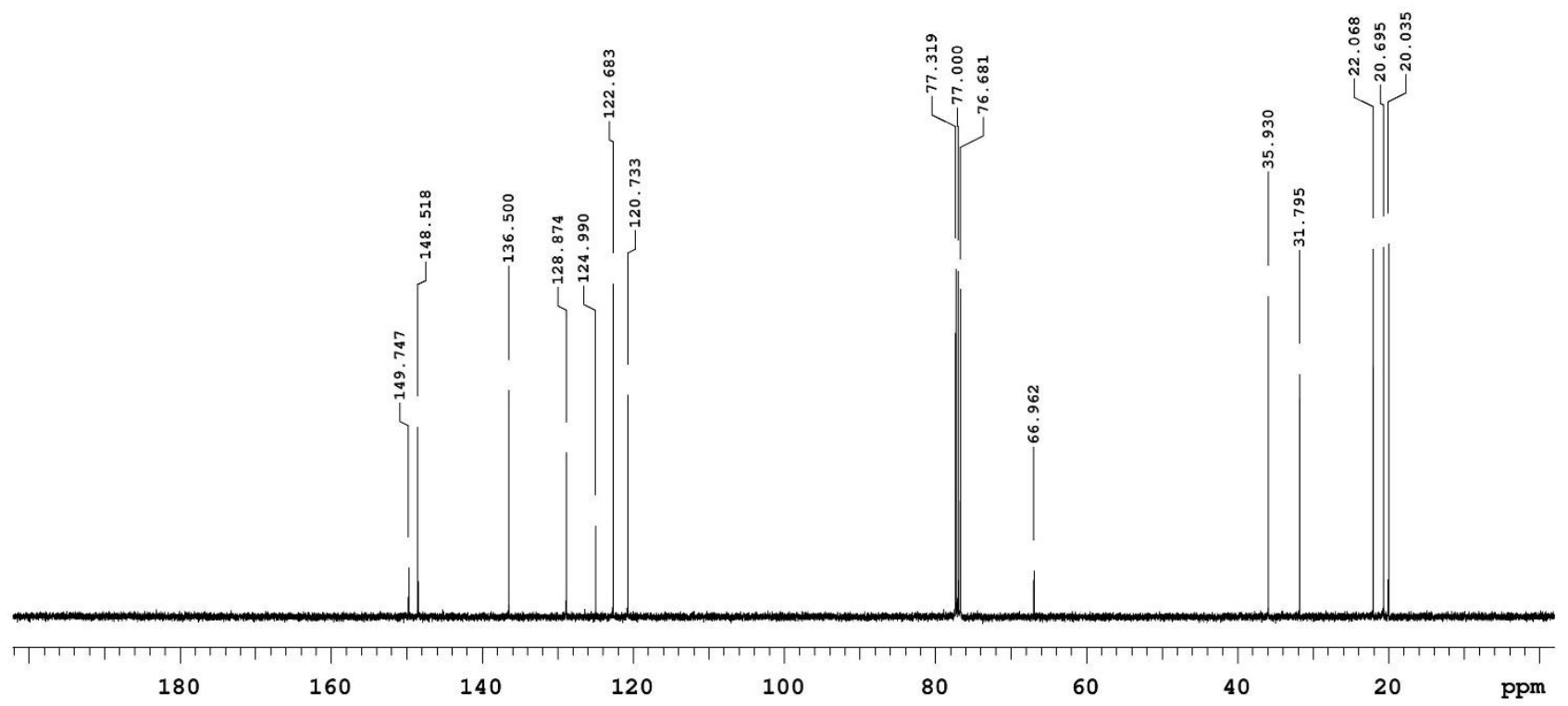


Sample Name: DA-283a

Solvent: cdc13

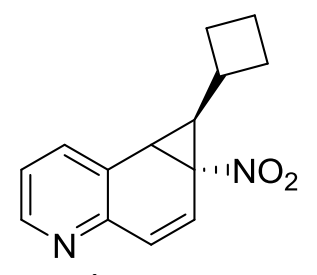

endo-3w

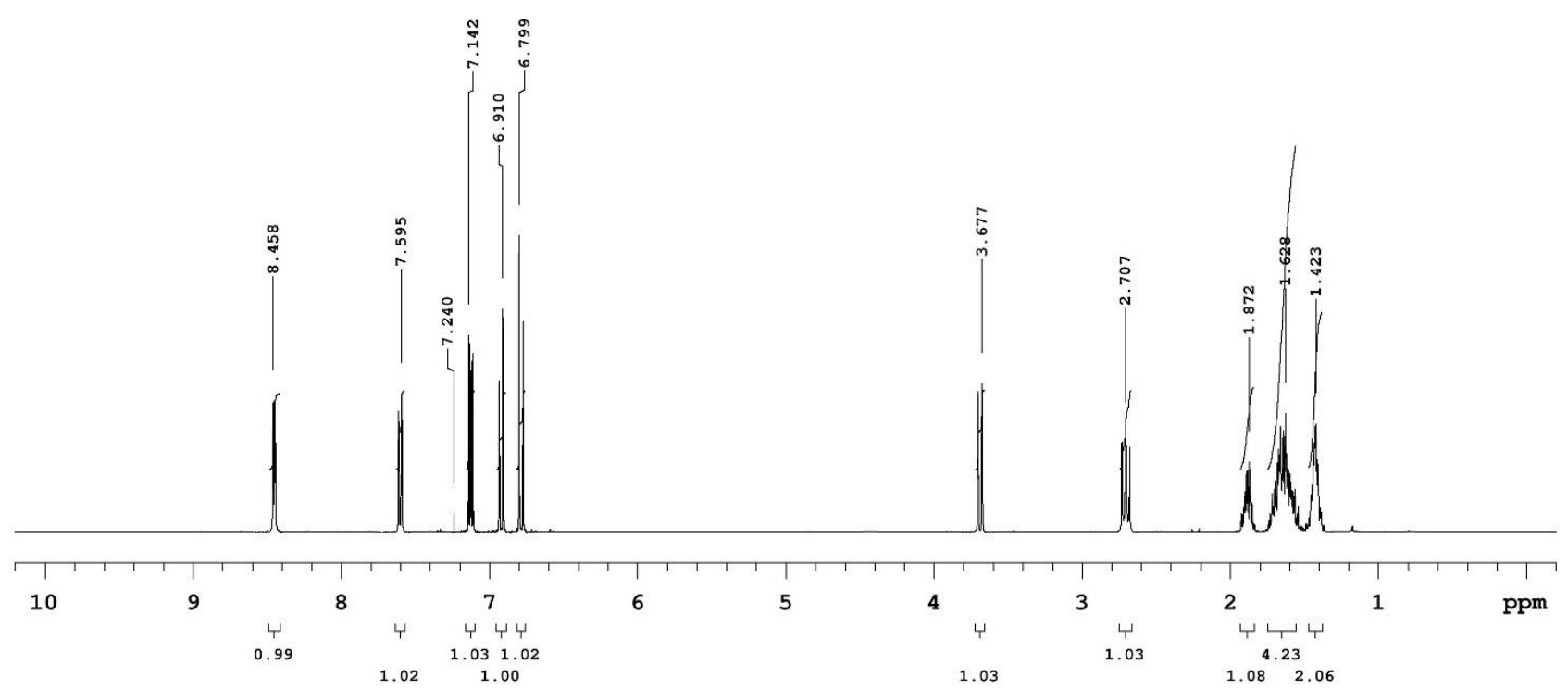

Sample Name: DA-283a

Solvent: cdc13

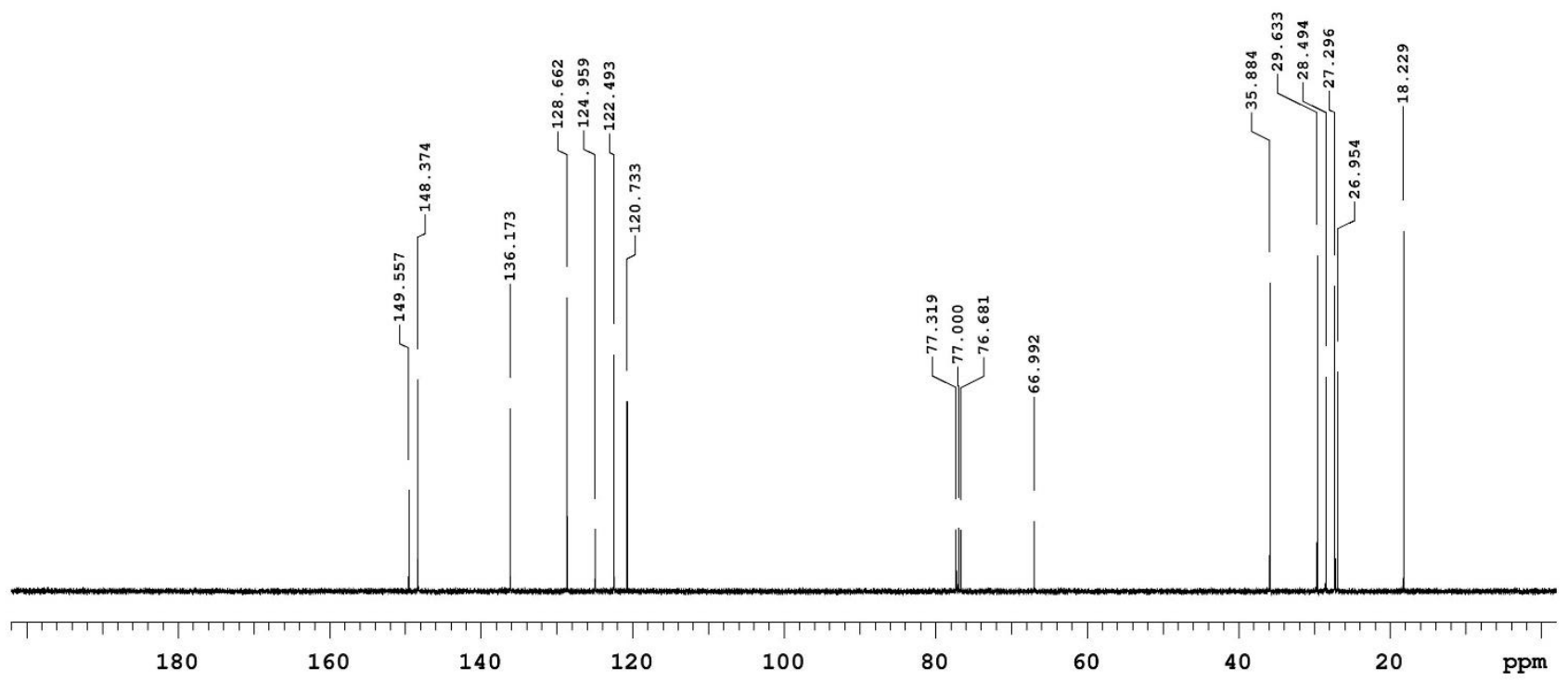


Sample Name: DA-282b2

Solvent: cdc13

(inO)

endo-3x

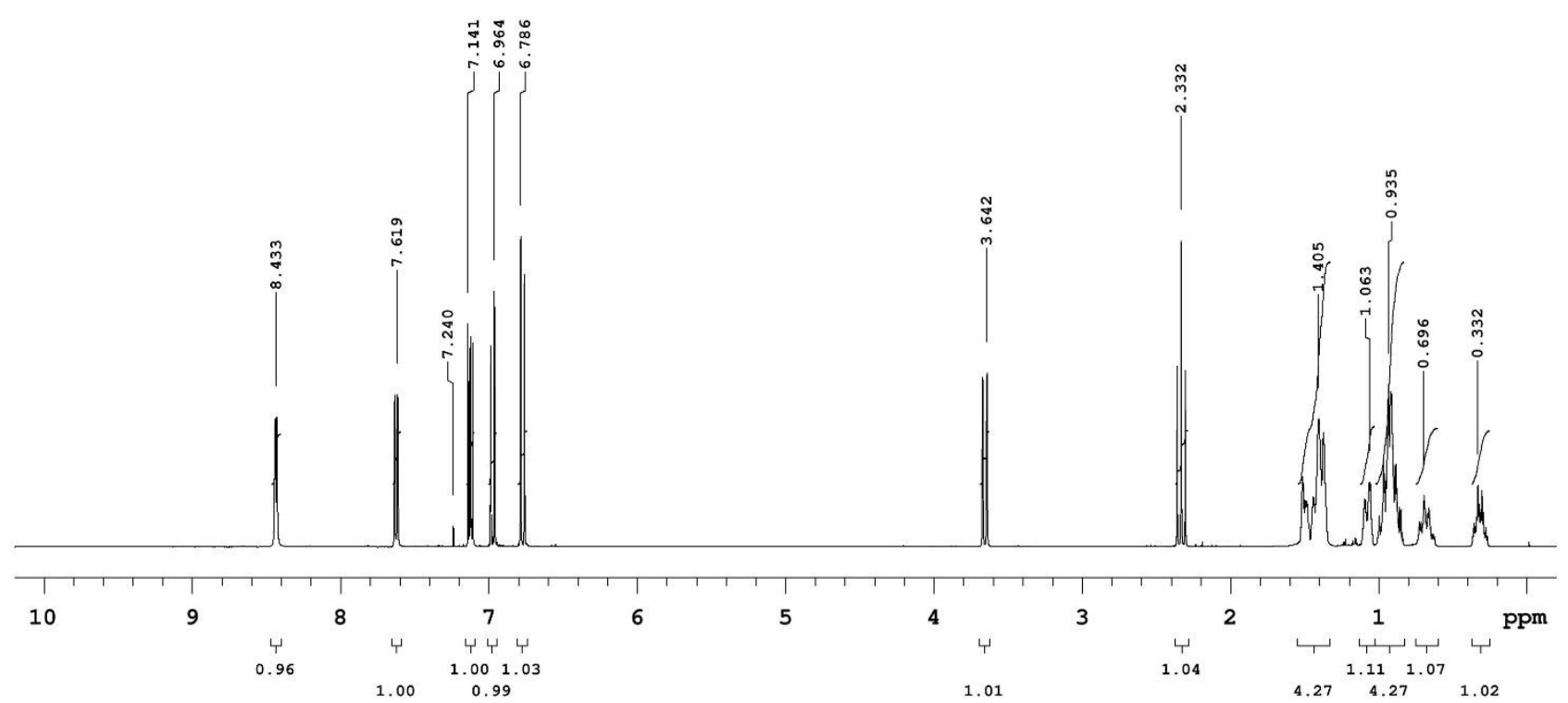

Sample Name: DA-282b2

Solvent: cdc13

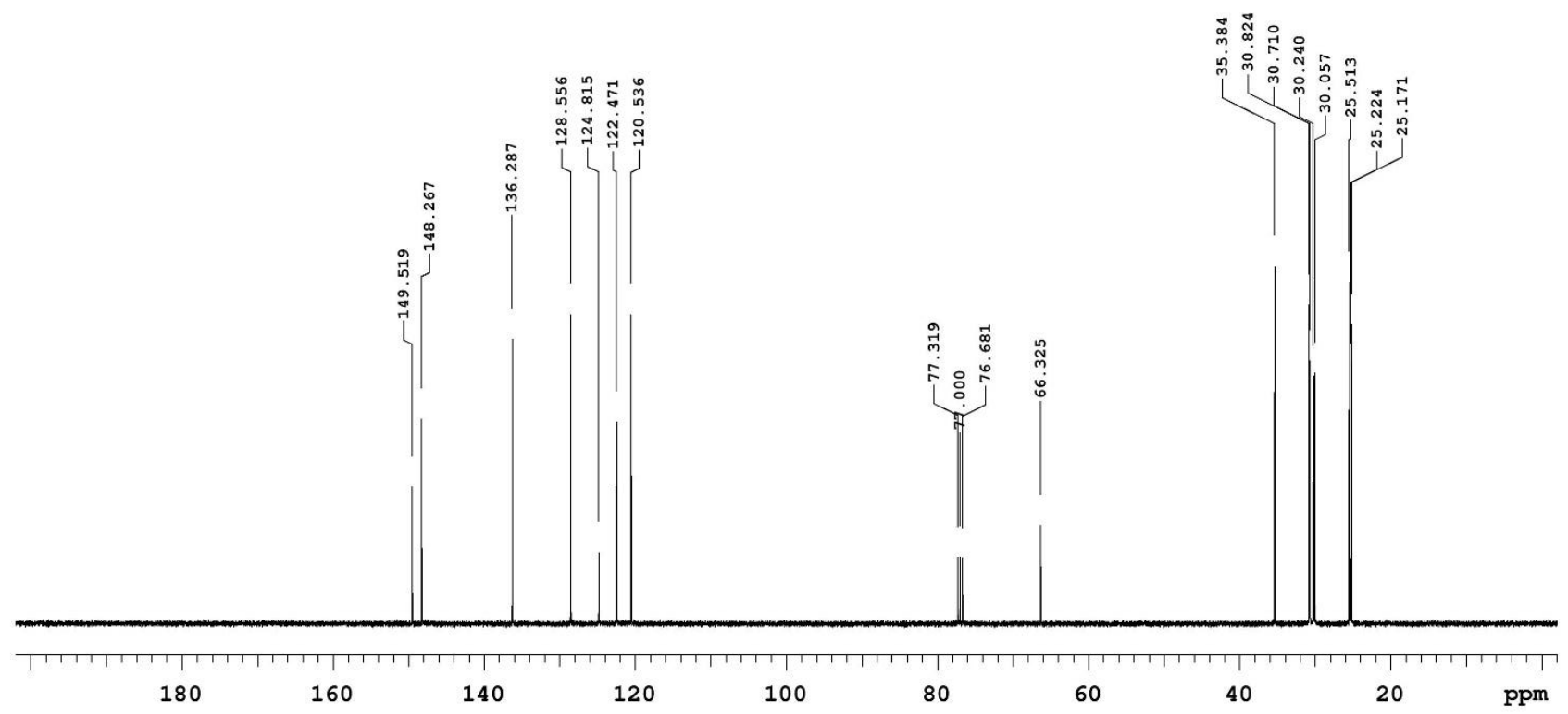


Sample Name: DA-282d

Solvent: cdc13

exo-3x

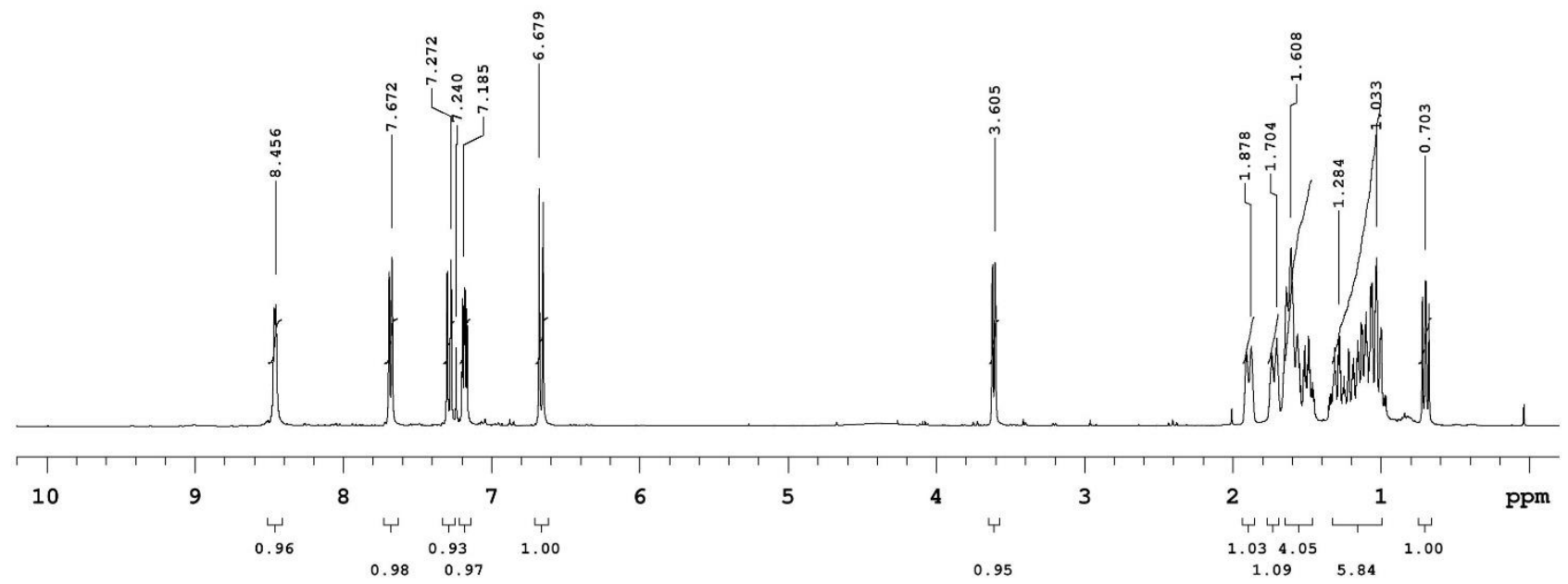

Sample Name: DA-282d

Solvent: cdc13

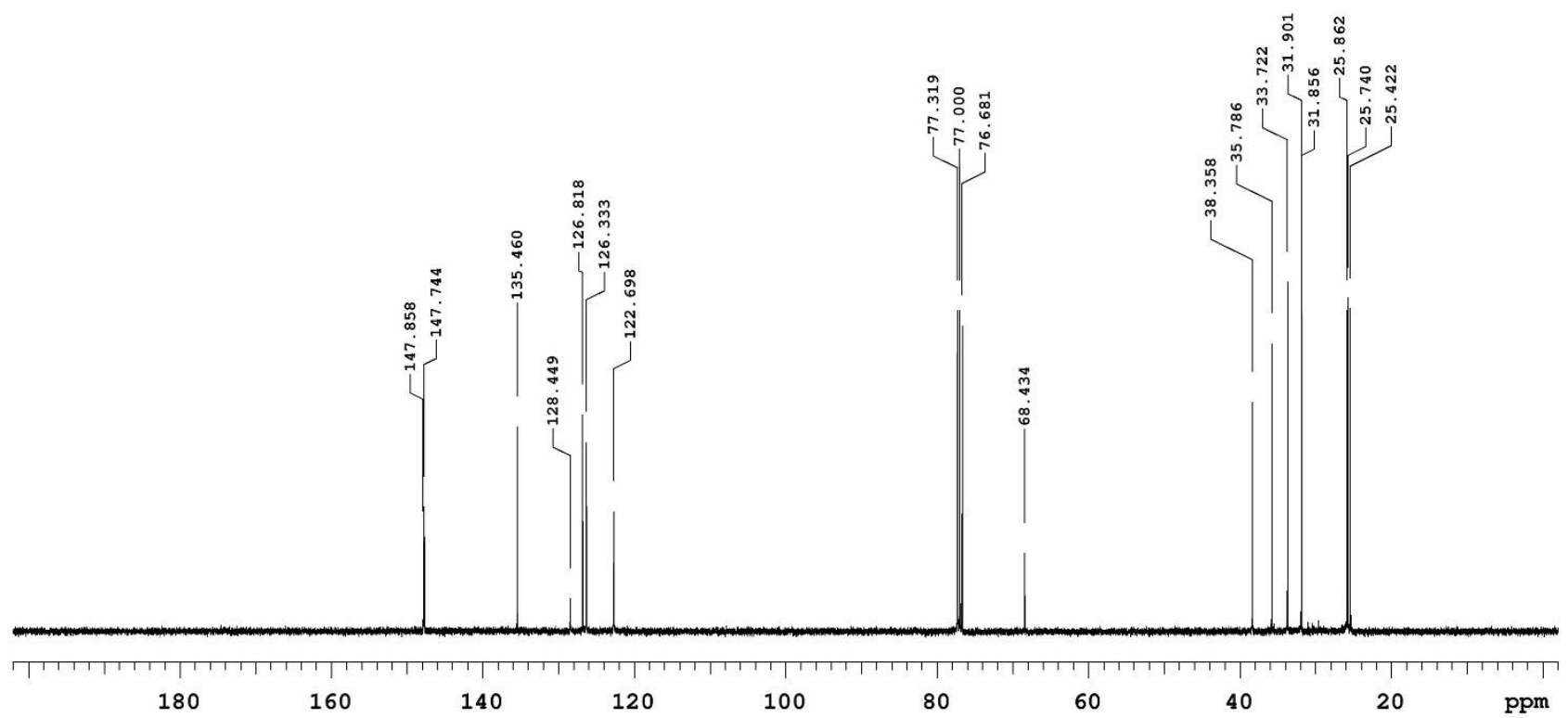


Sample Name: DA-288aa

Solvent: cdc13

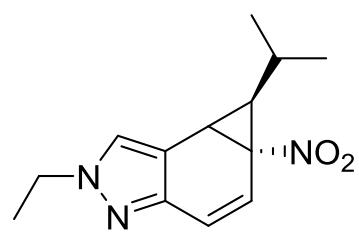

endo-3y

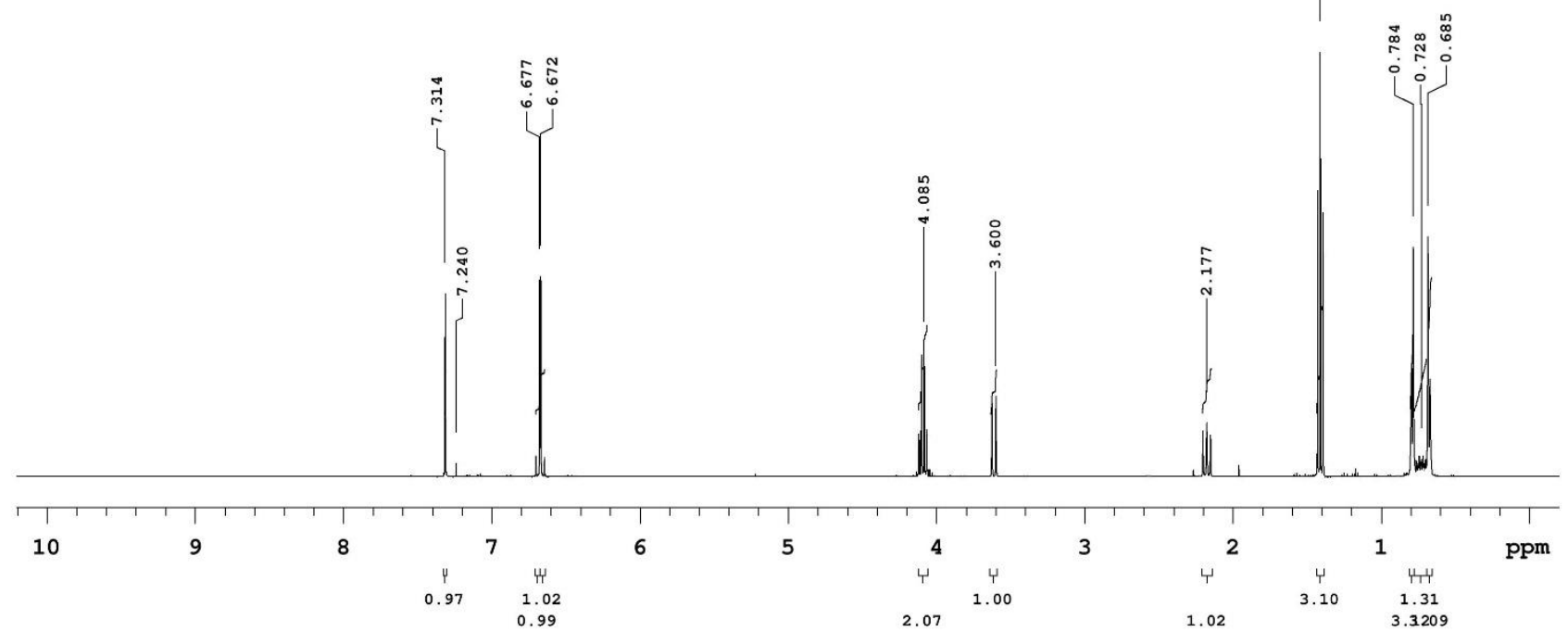

Sample Name: DA-288aa

Solvent: $\operatorname{cdcl} 3$

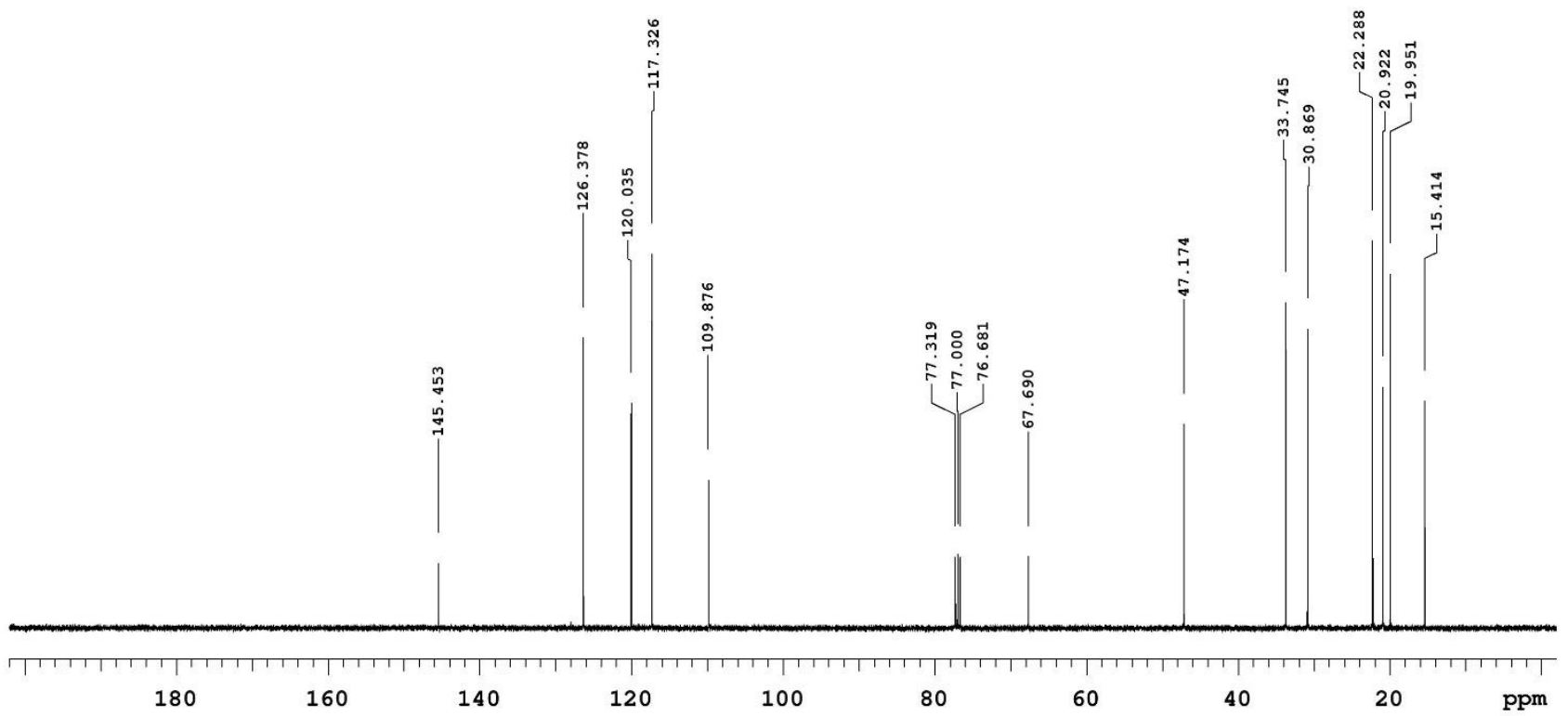


Sample Name: DA-287d

Solvent: cdc13

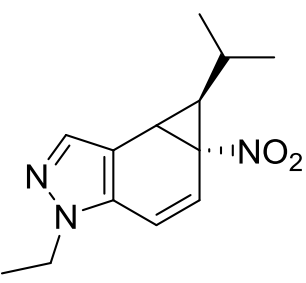

endo-3z

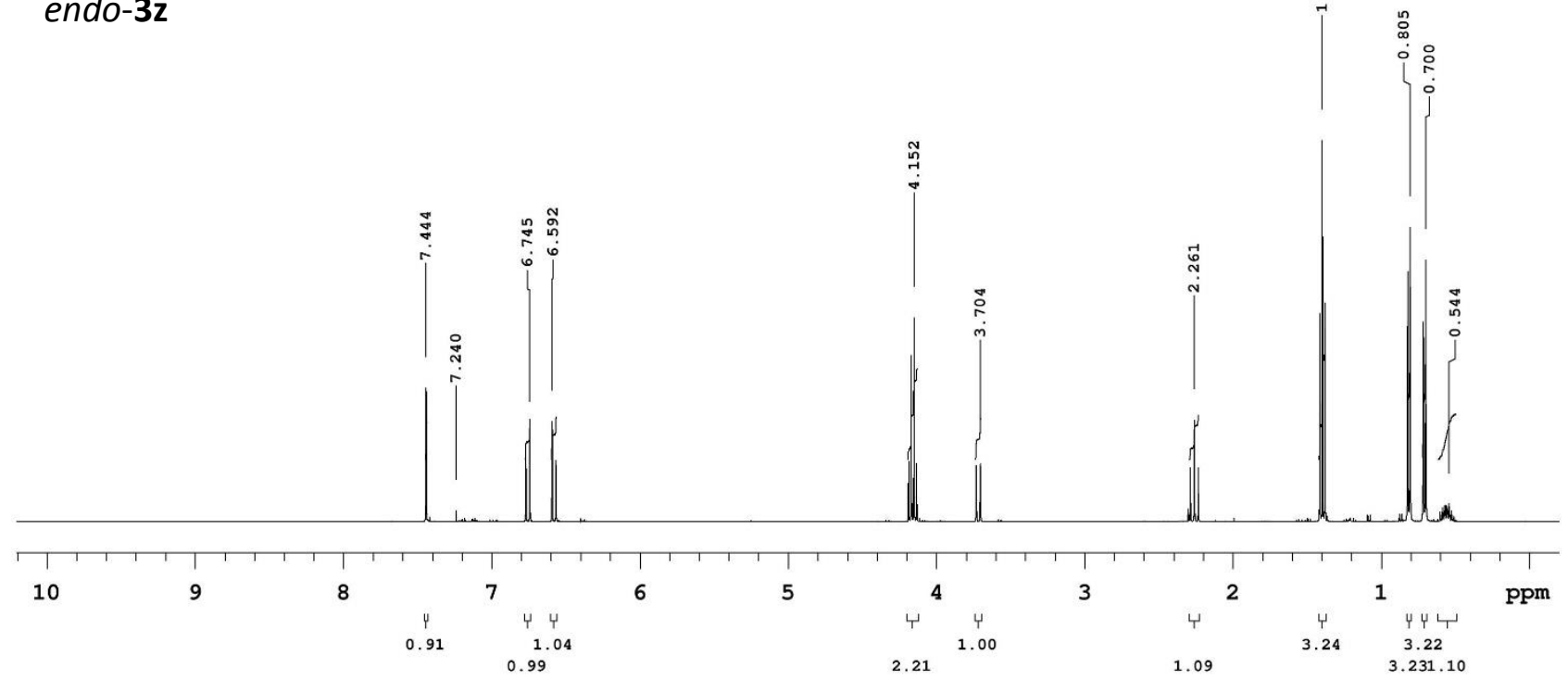

Sample Name: DA-287d

Solvent: cdc13

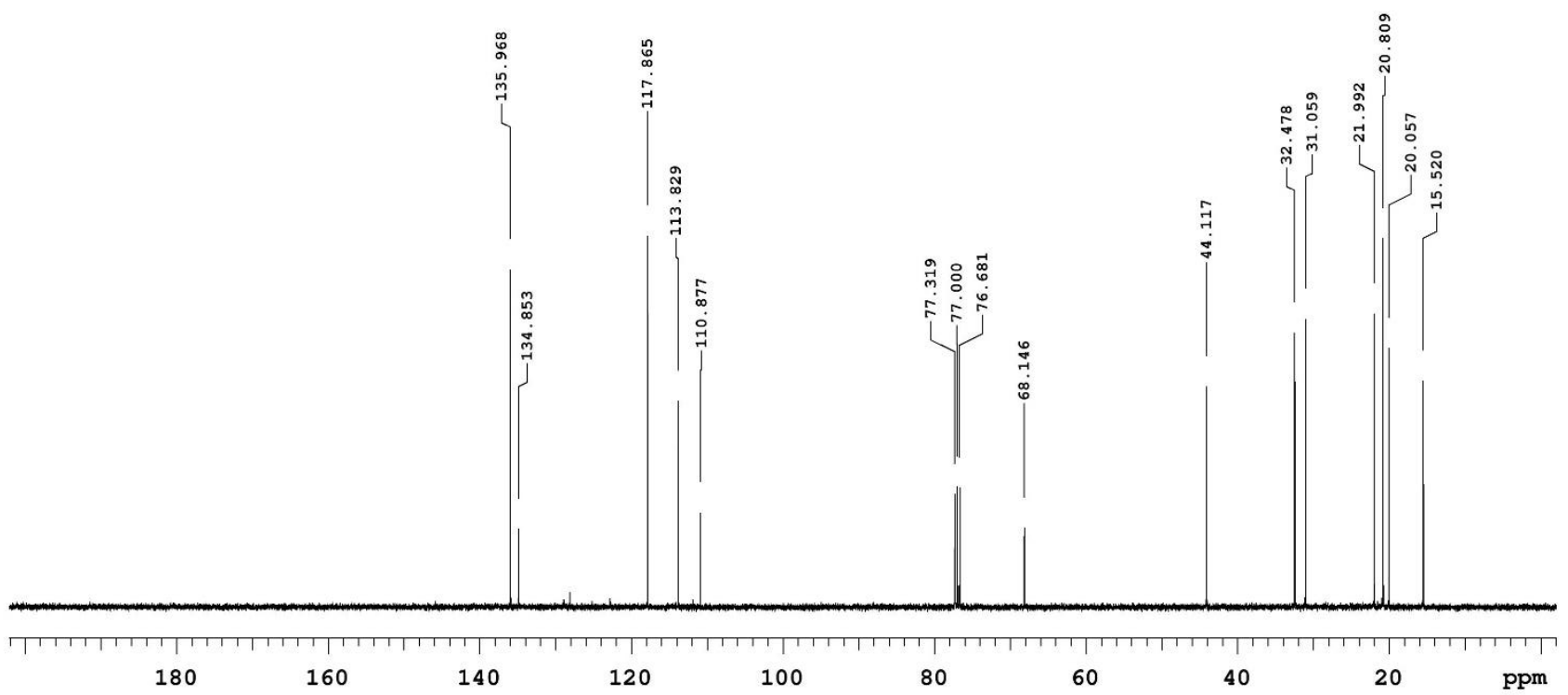


Sample Name: DA-312C

Solvent: cdc13
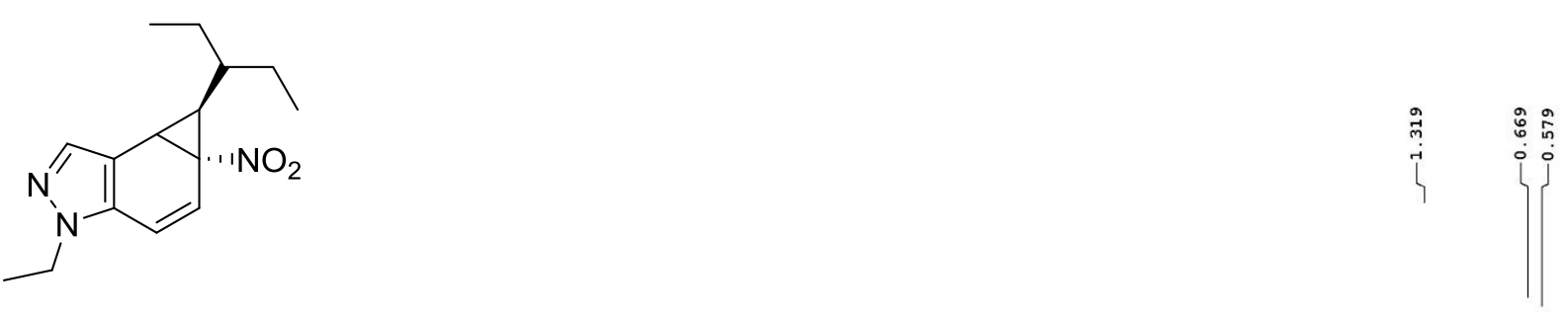

\section{endo-3aa}

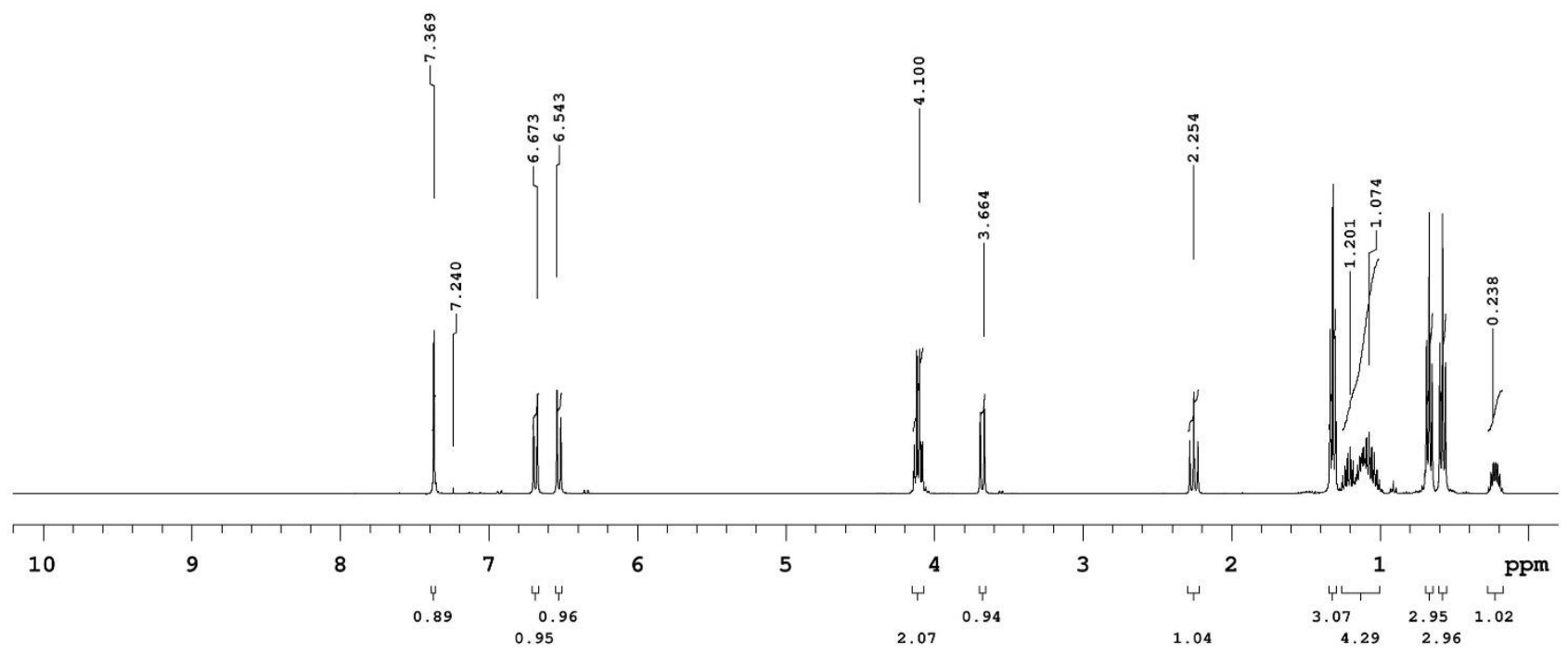

Sample Name: DA-312c

Solvent: cdc13

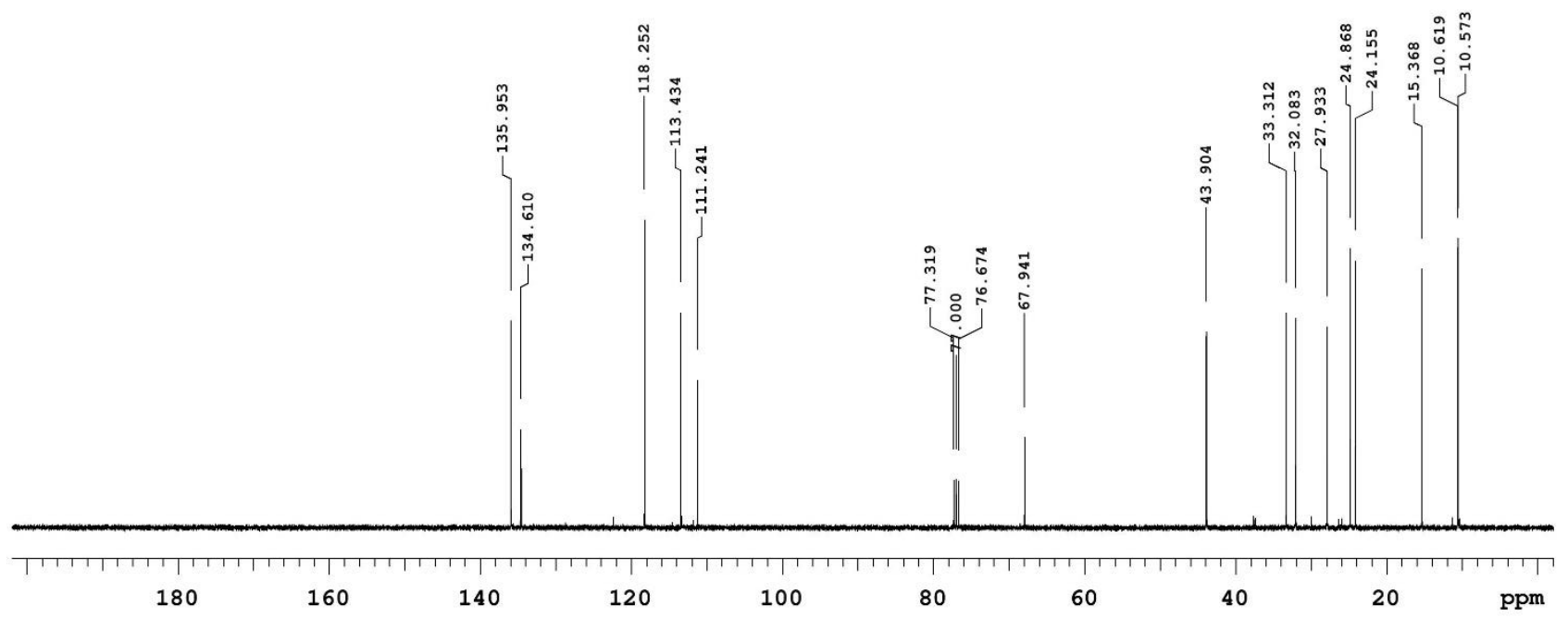


Sample Name: DA-244C

Solvent: cdc13

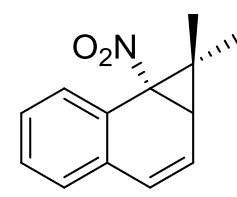

$3 a b$

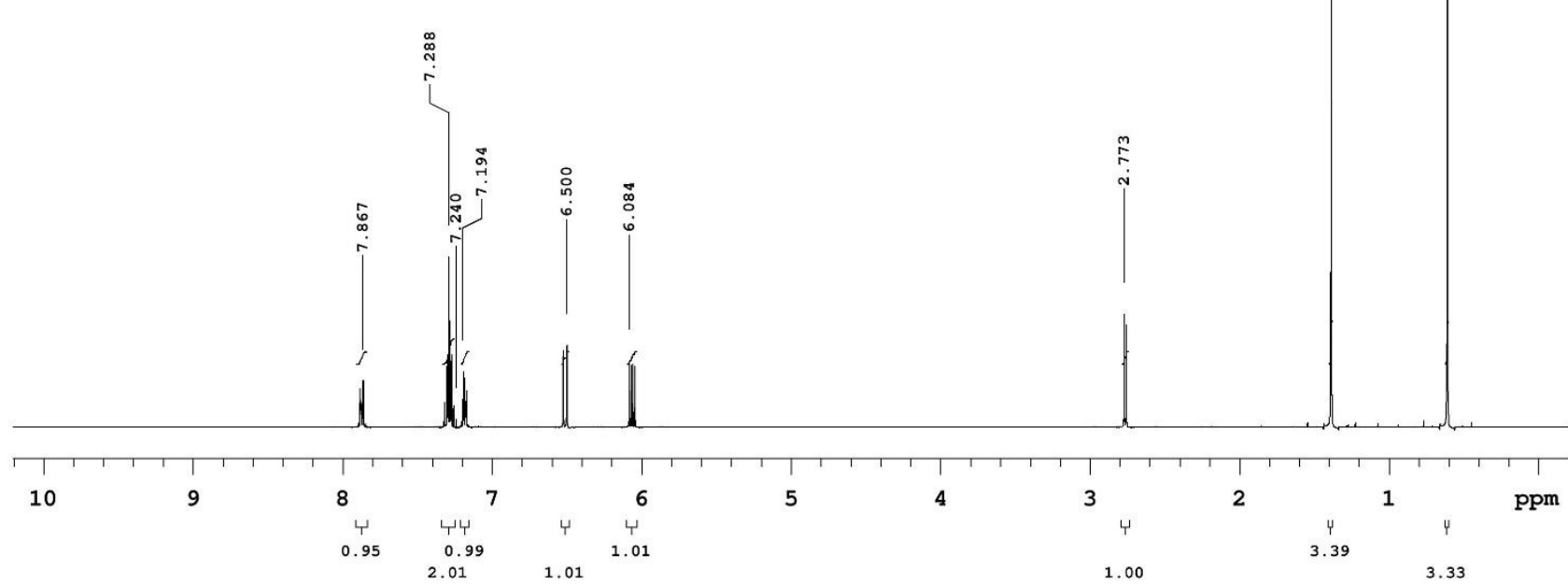

Sample Name: DA-244C

Solvent: cdc13

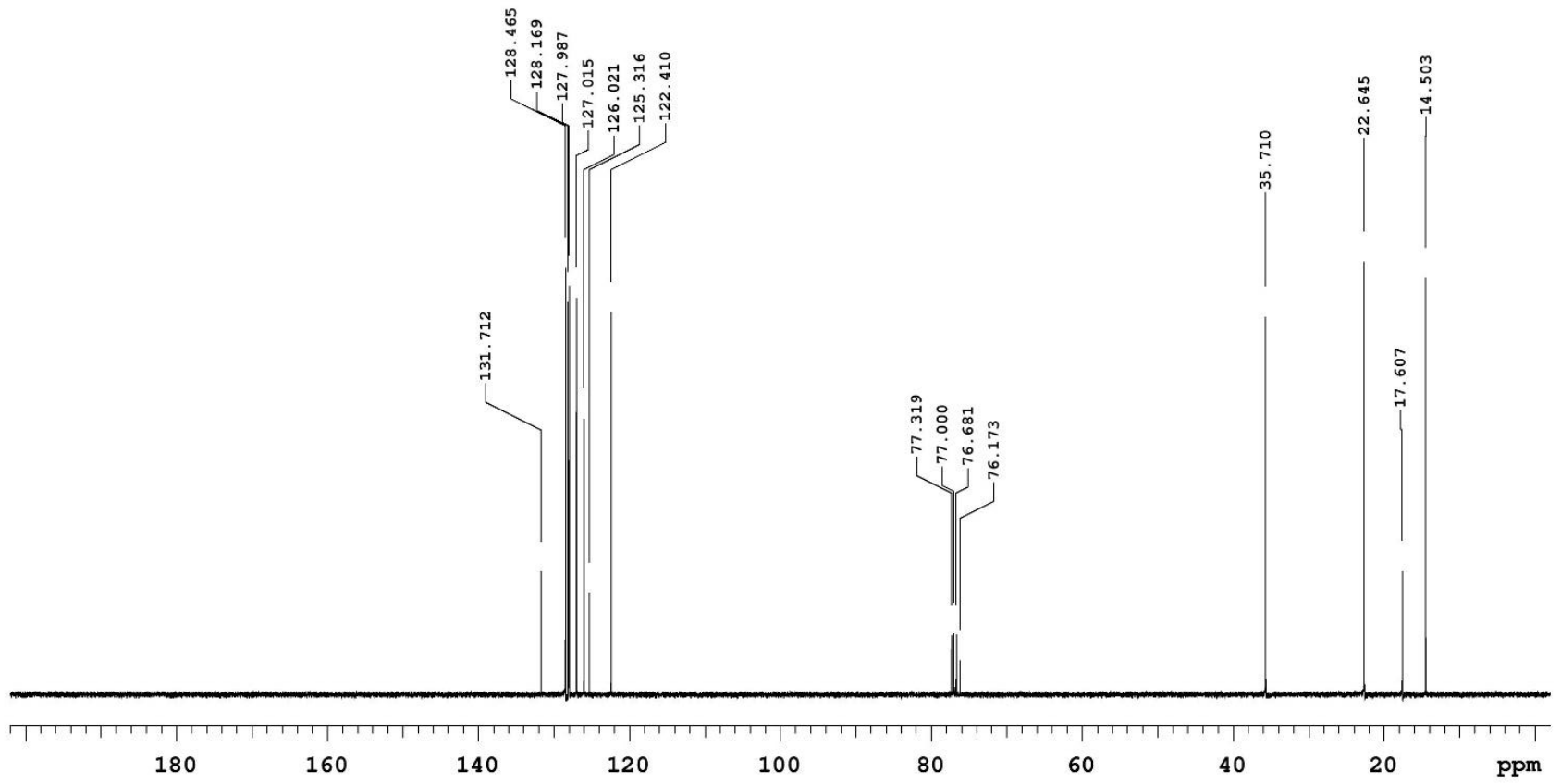



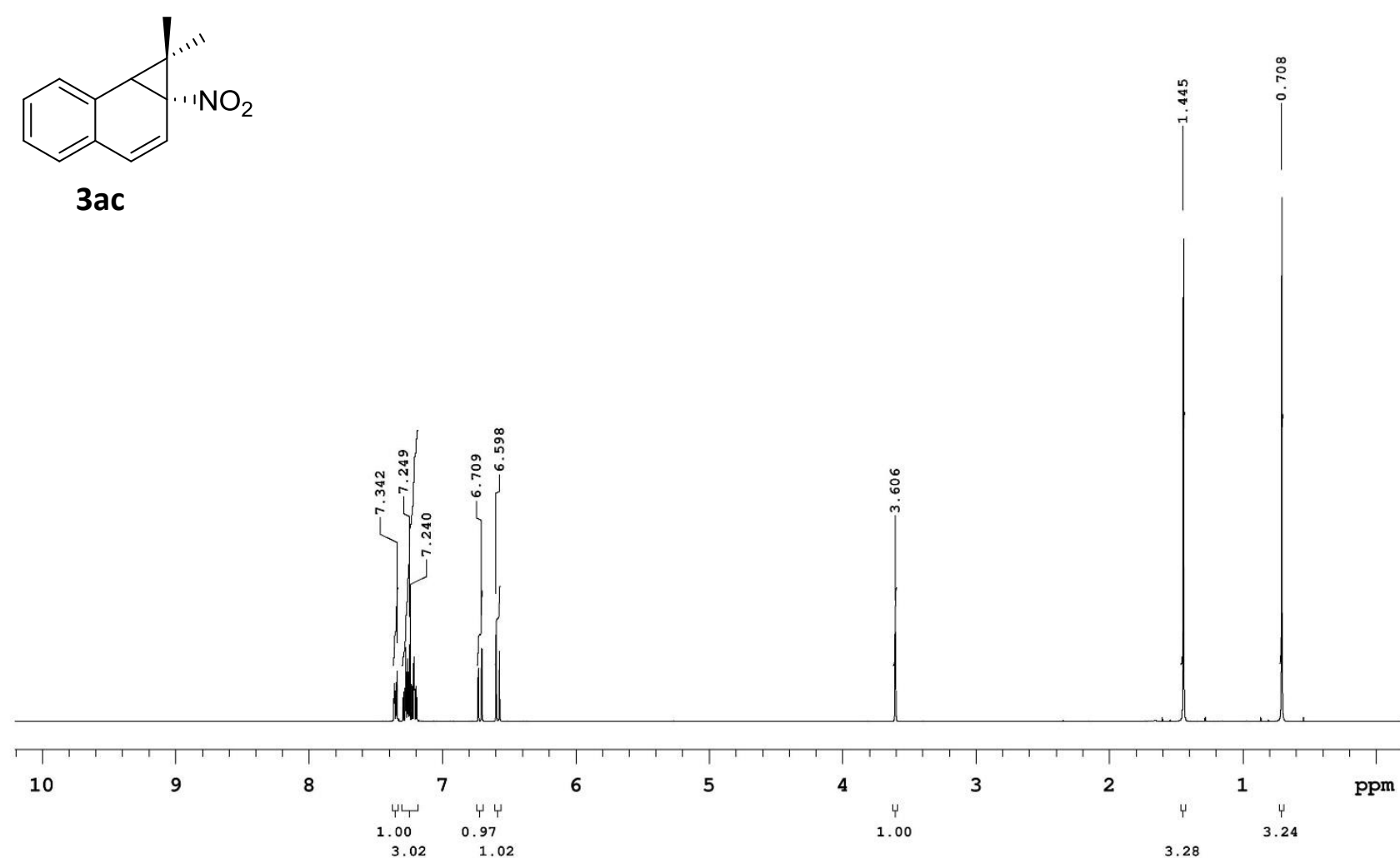

Sample Name: DA-293b

Solvent: cdc13

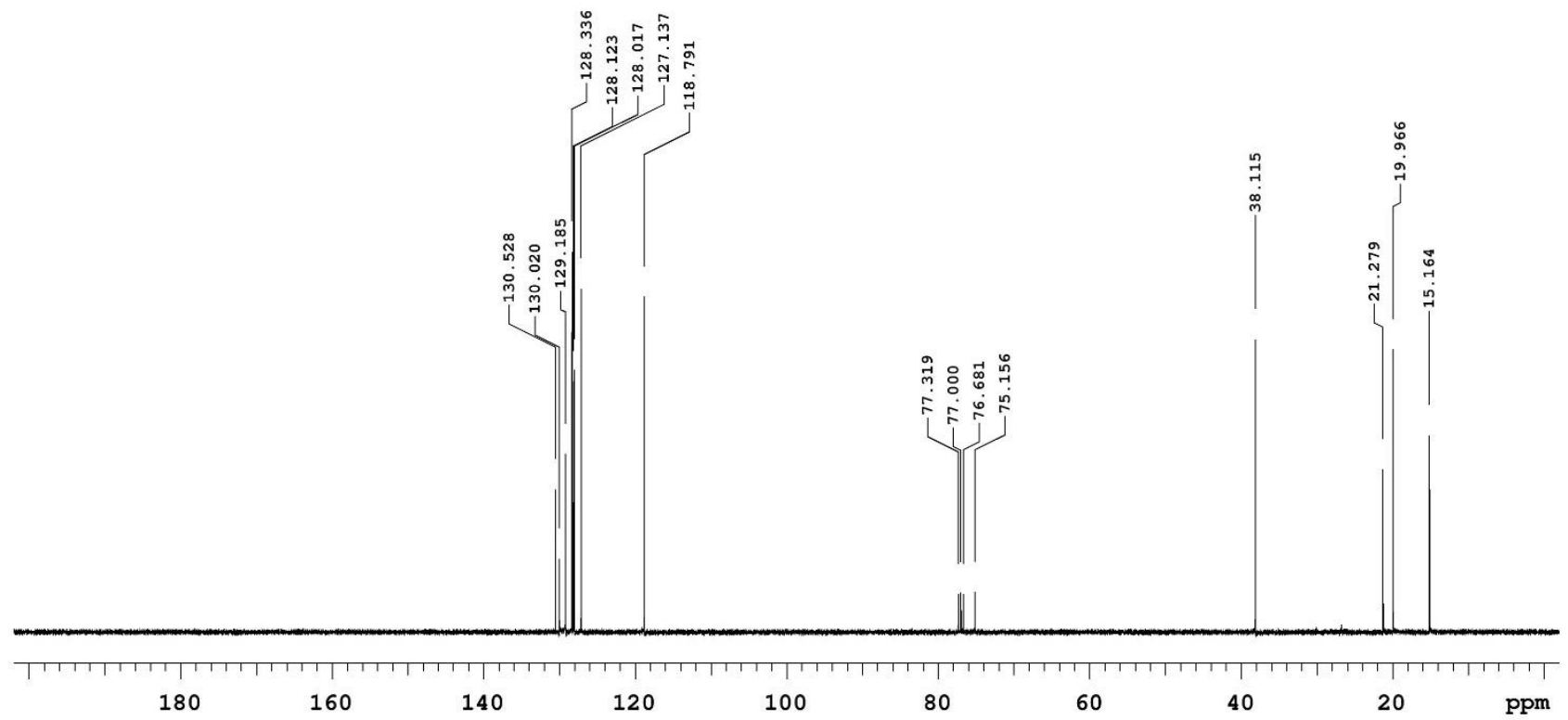



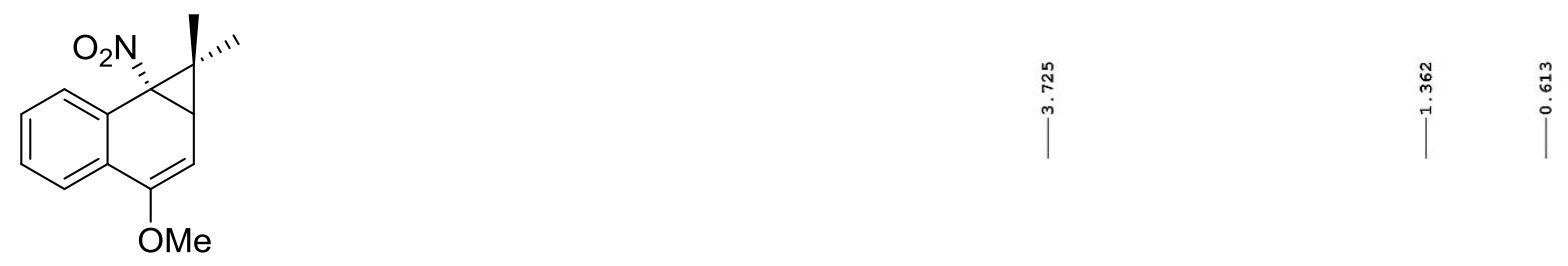

3ad

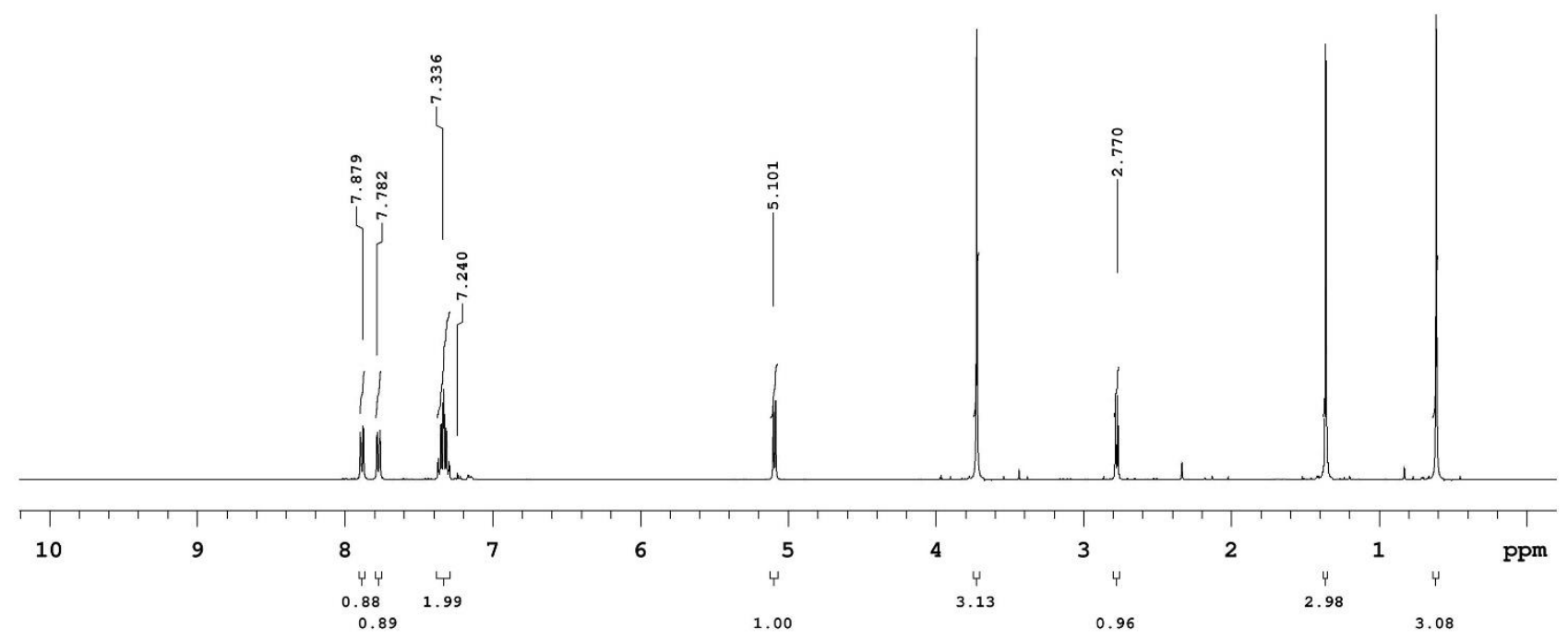

Sample Name: DA-294b

Solvent: cdc13

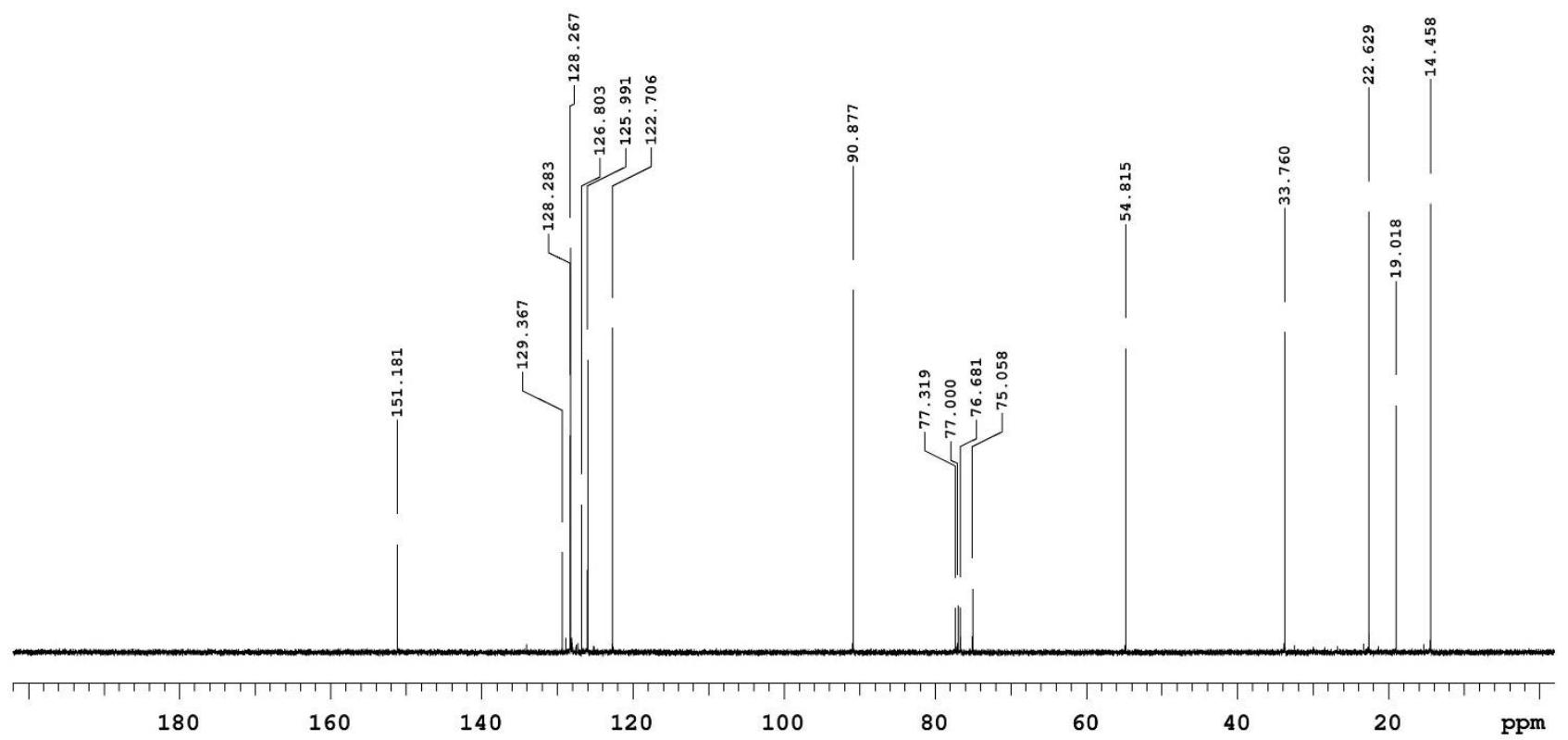



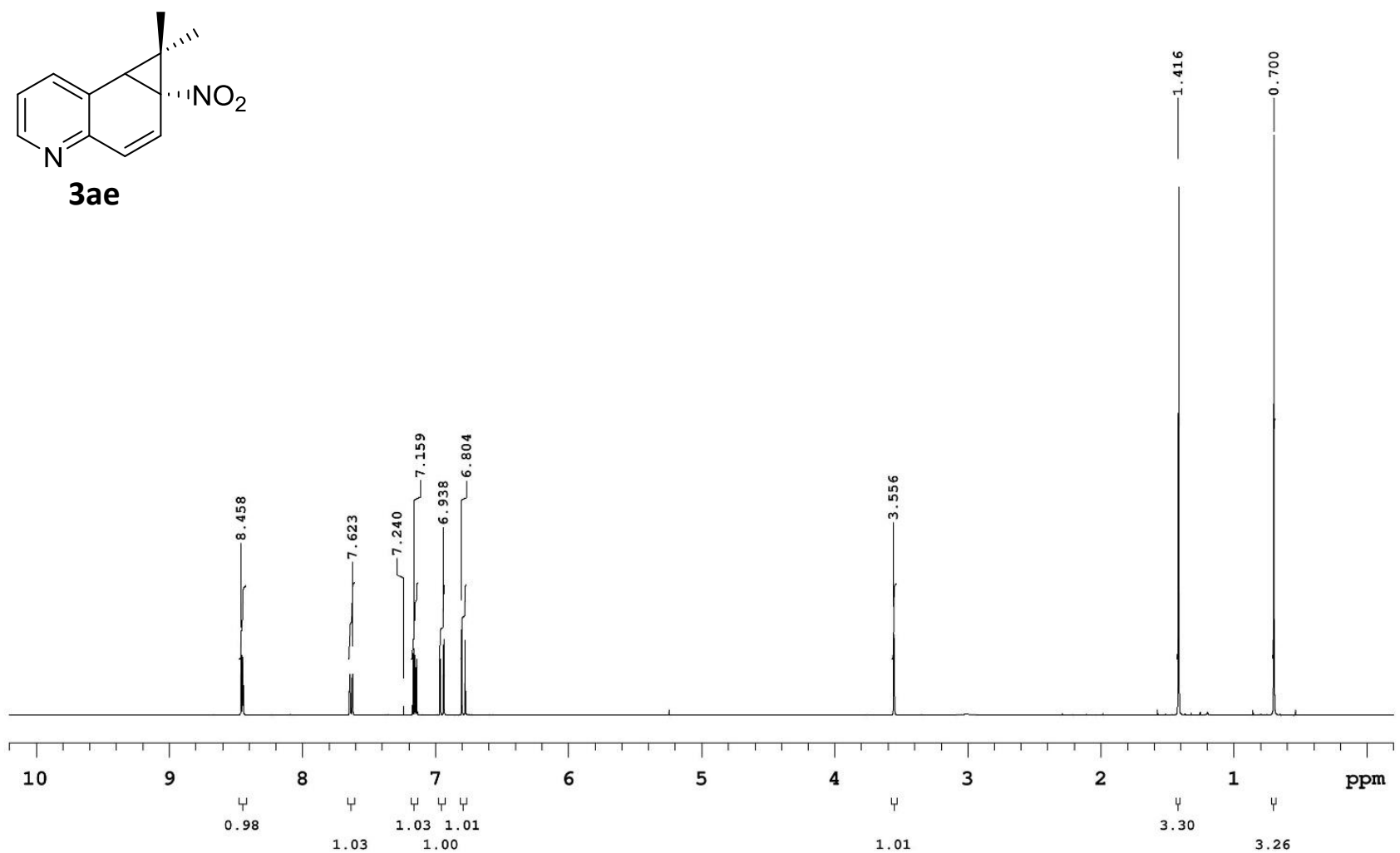

Sample Name: DA-295bc Solvent: $\operatorname{cdc13}$

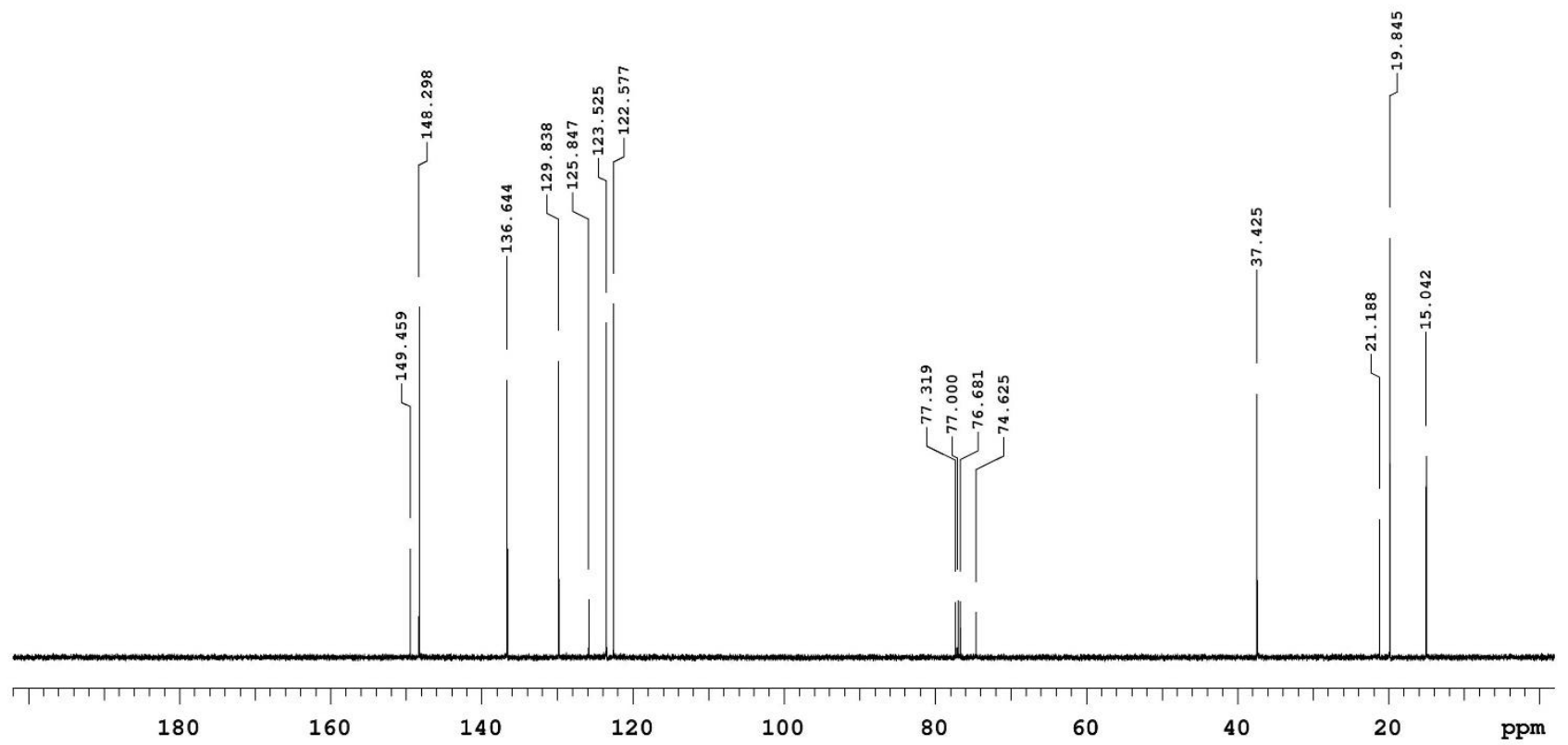


Sample Name: DA-296b

Solvent: cdc13

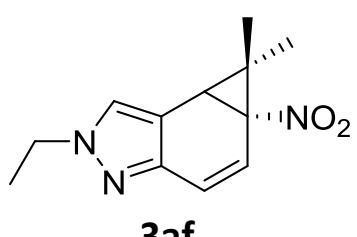

$\mathrm{NO}_{2}$

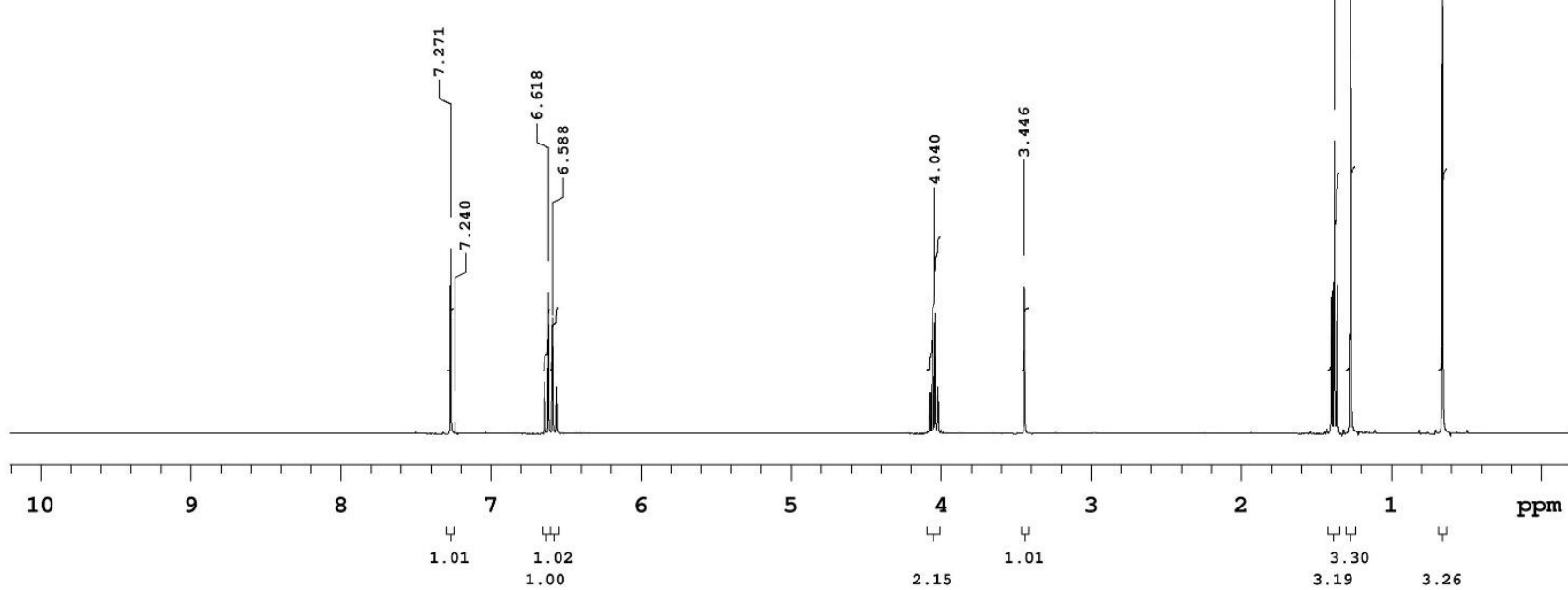

Sample Name: DA-296b

Solvent: cdc13

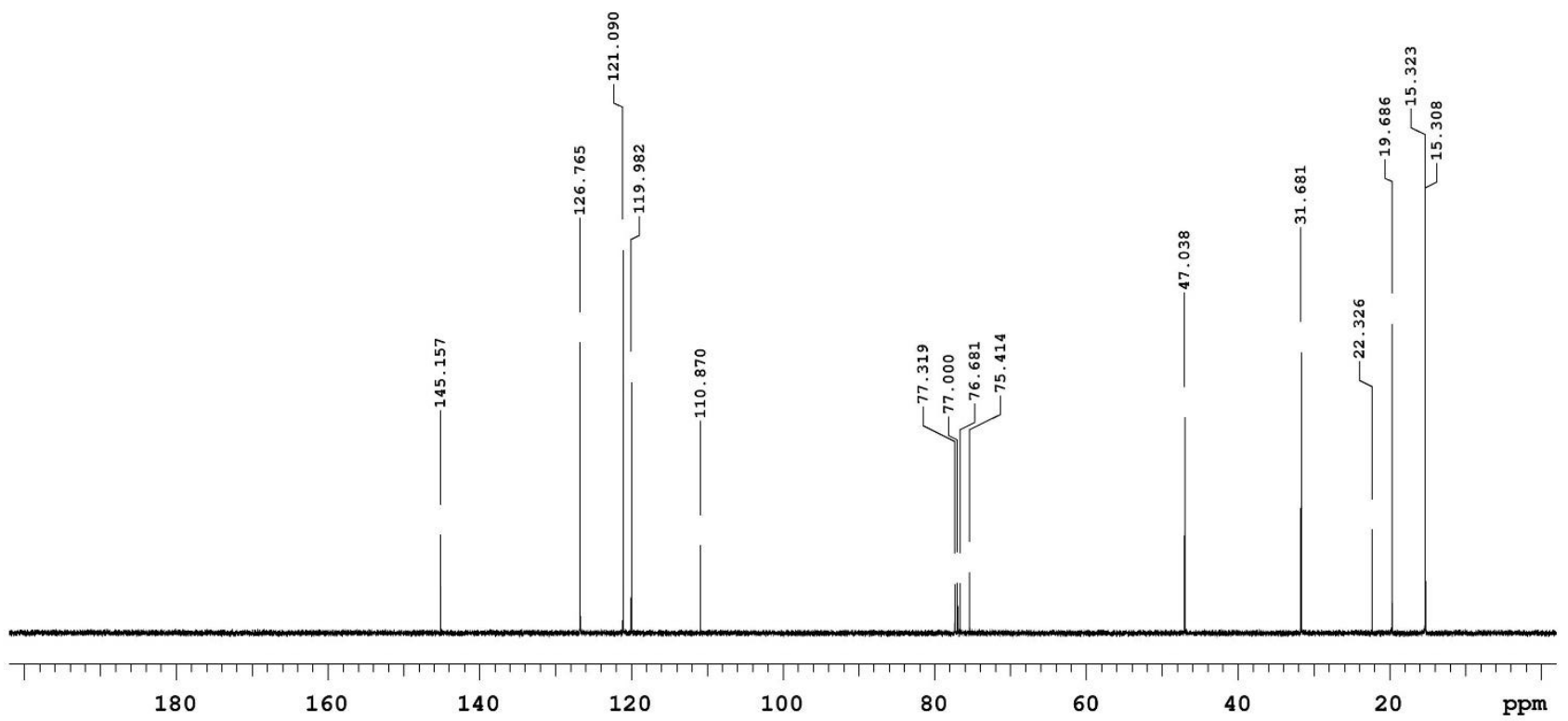



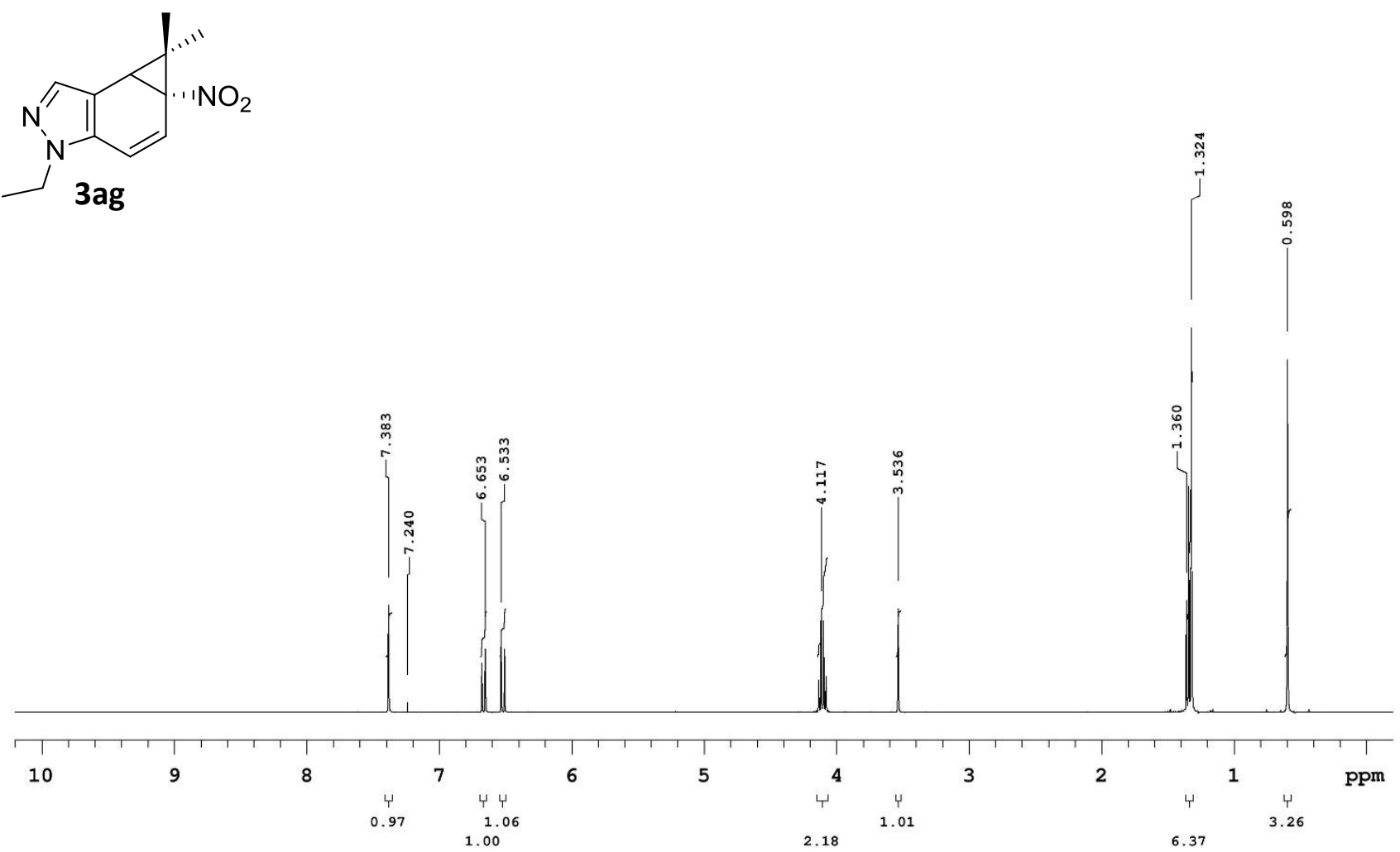

Sample Name: DA-310c

Solvent: cdc13

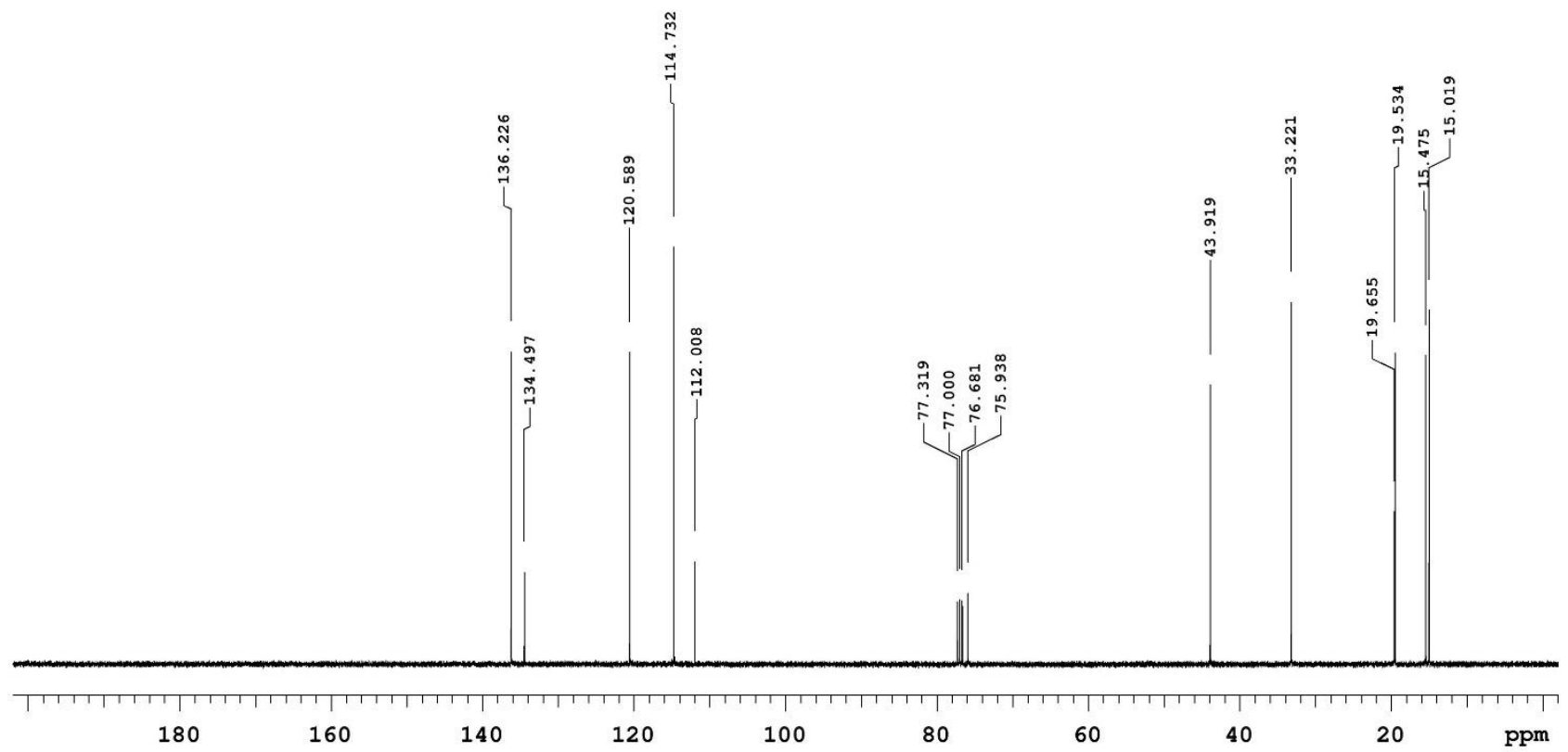


<smiles>Cc1ccc2ccccc2c1[N+](=O)[O-]</smiles>

$4 a$
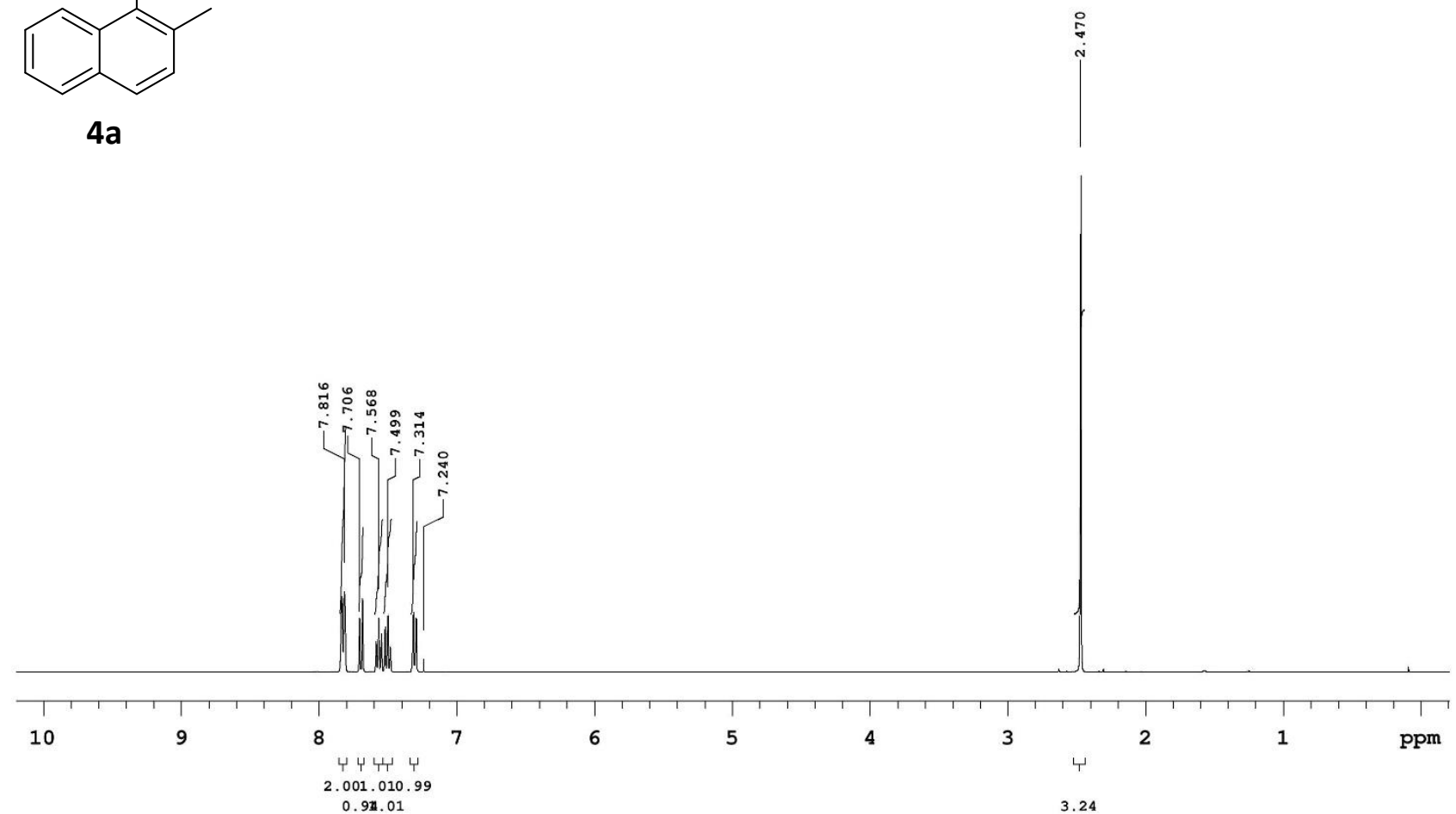

Sample Name: $\mathrm{DA}-020$ Solvent: cdcl3

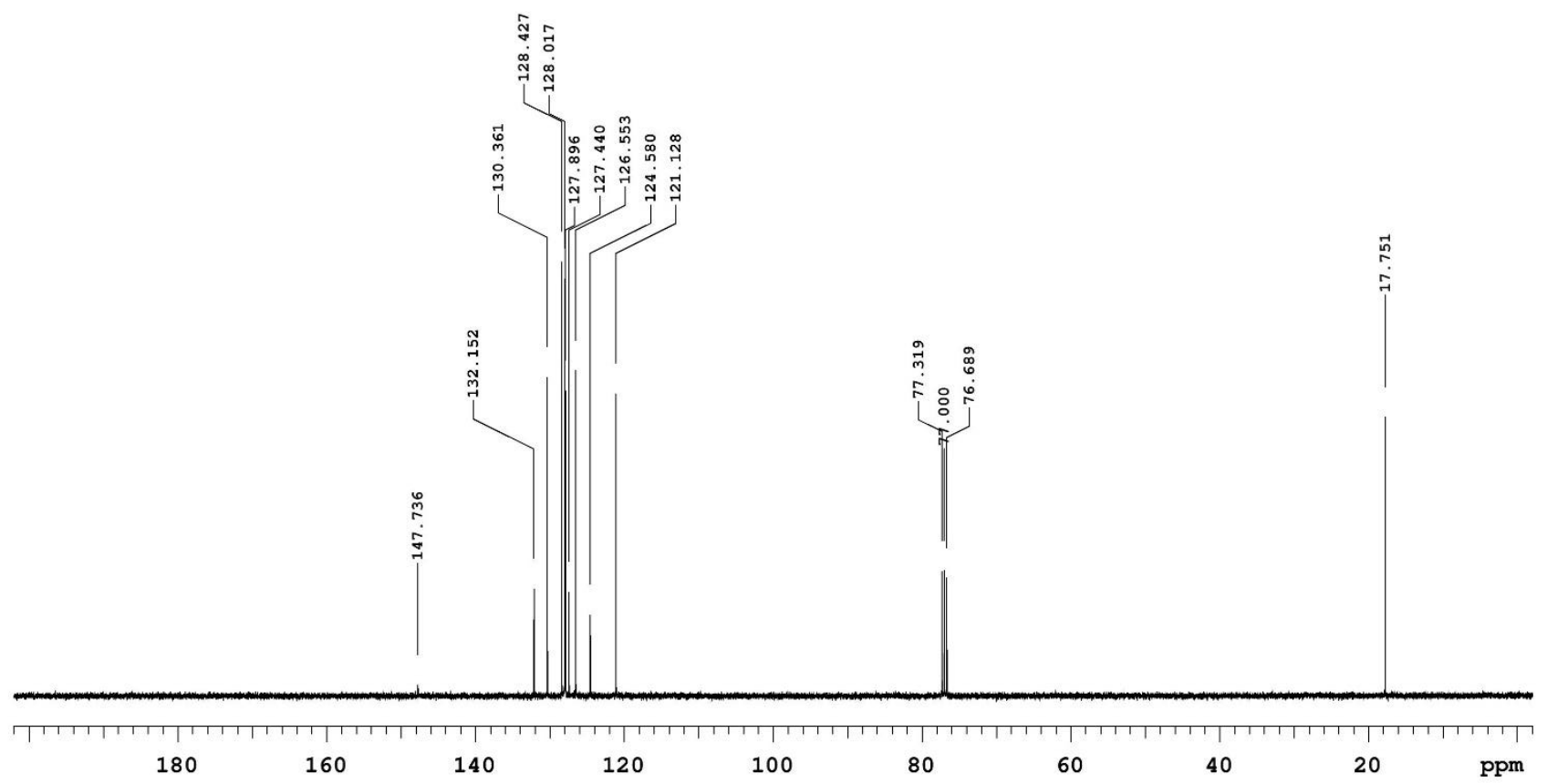


Sample Name: DA-019a

Solvent: cdc13<smiles>CCc1ccc2ccccc2c1[N+](=O)[O-]</smiles>

4b

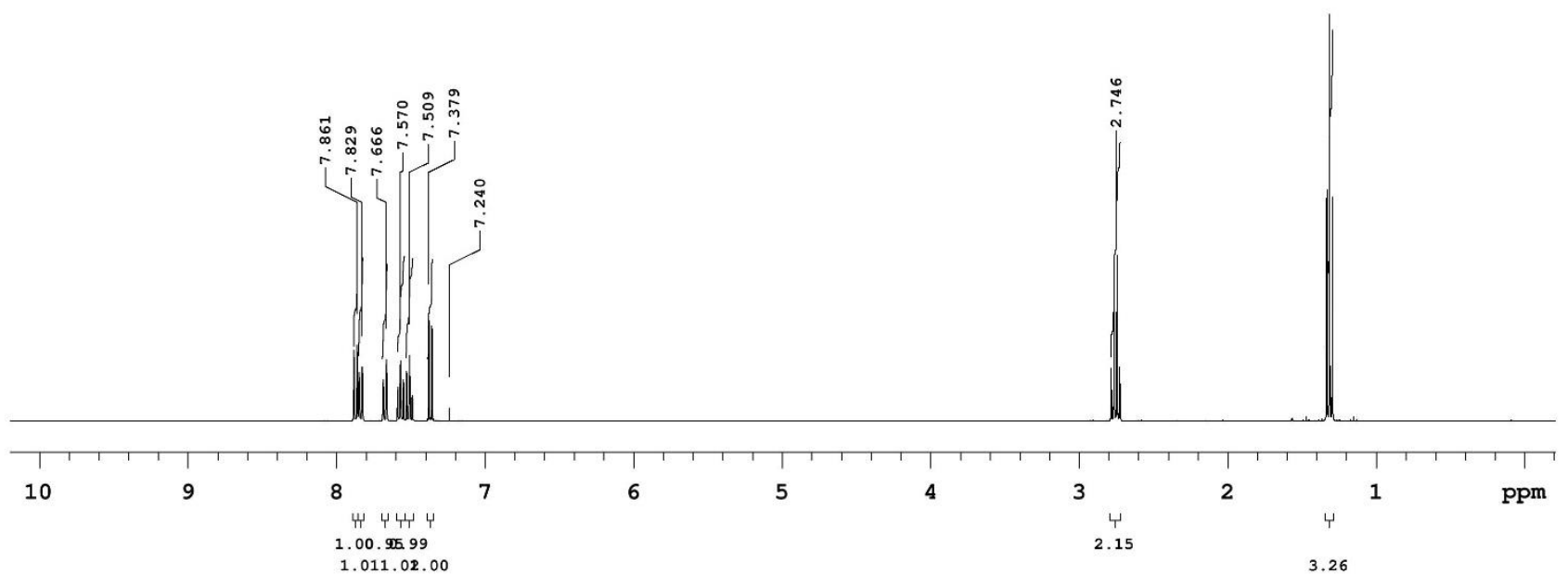

Sample Name: DA-019a

Solvent: $\operatorname{cdcl} 3$

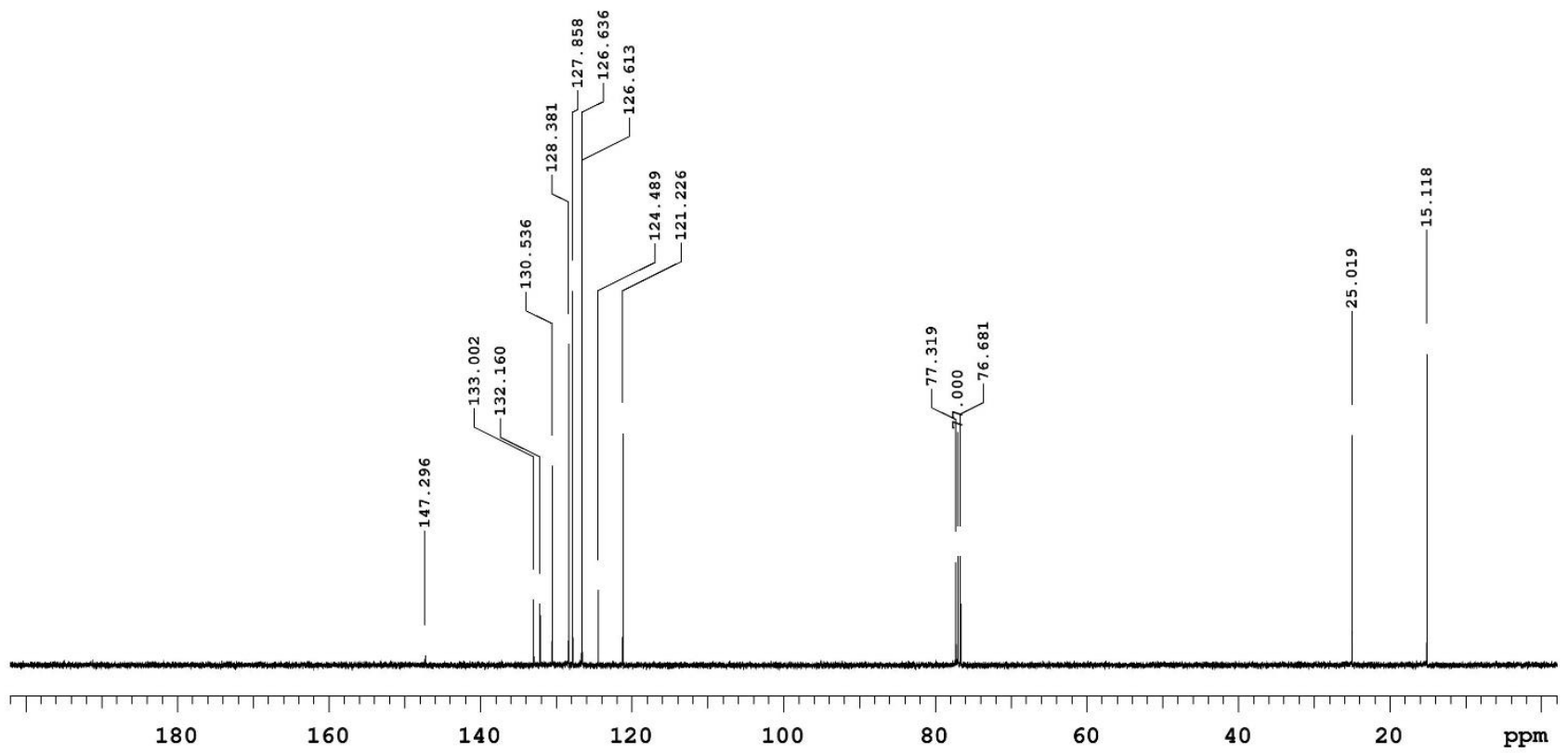



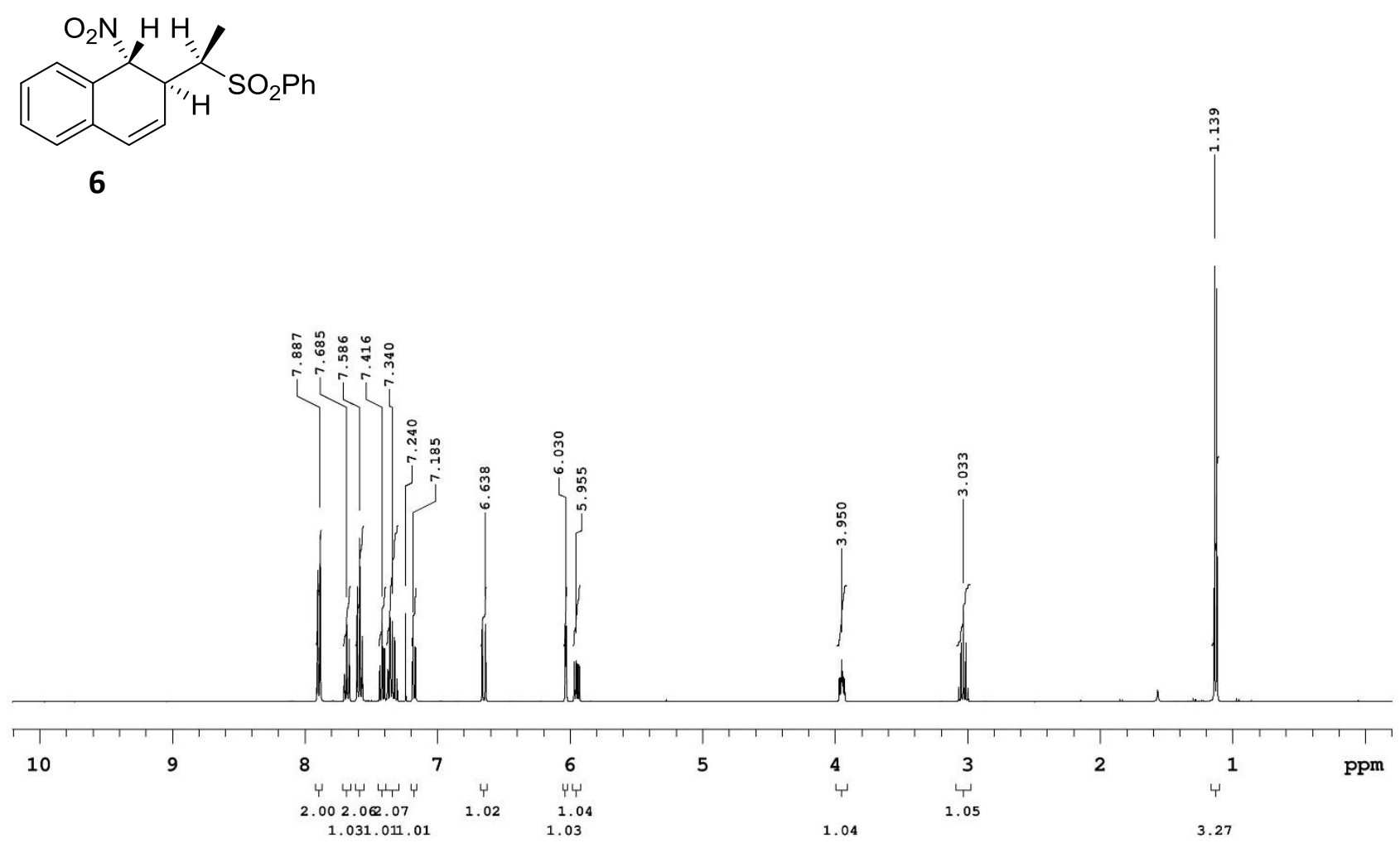

Sample Name: DA-186c-cryst Solvent: $\operatorname{cdcl} 3$

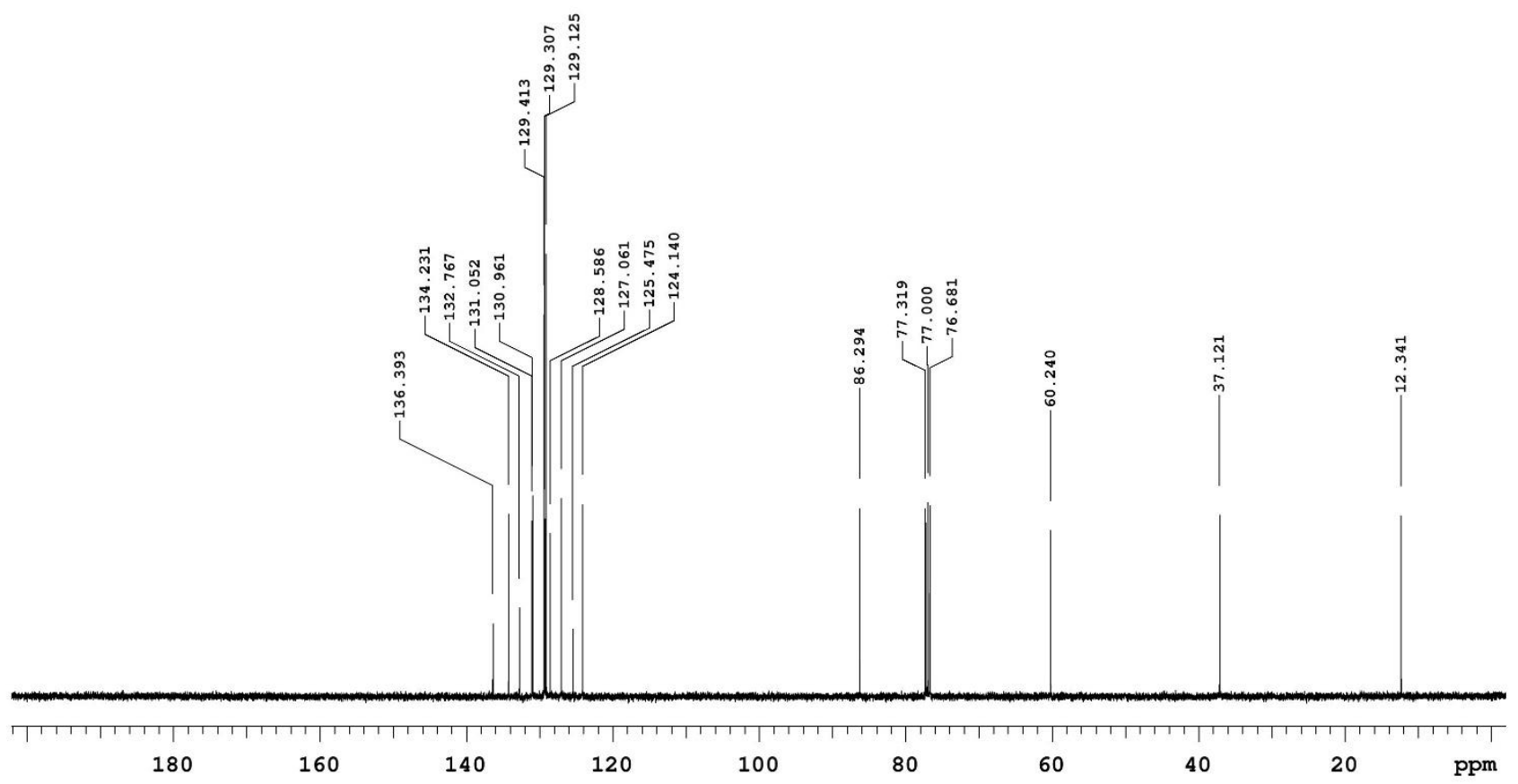


Sample Name: DA-249-extr

Solvent: cdc13
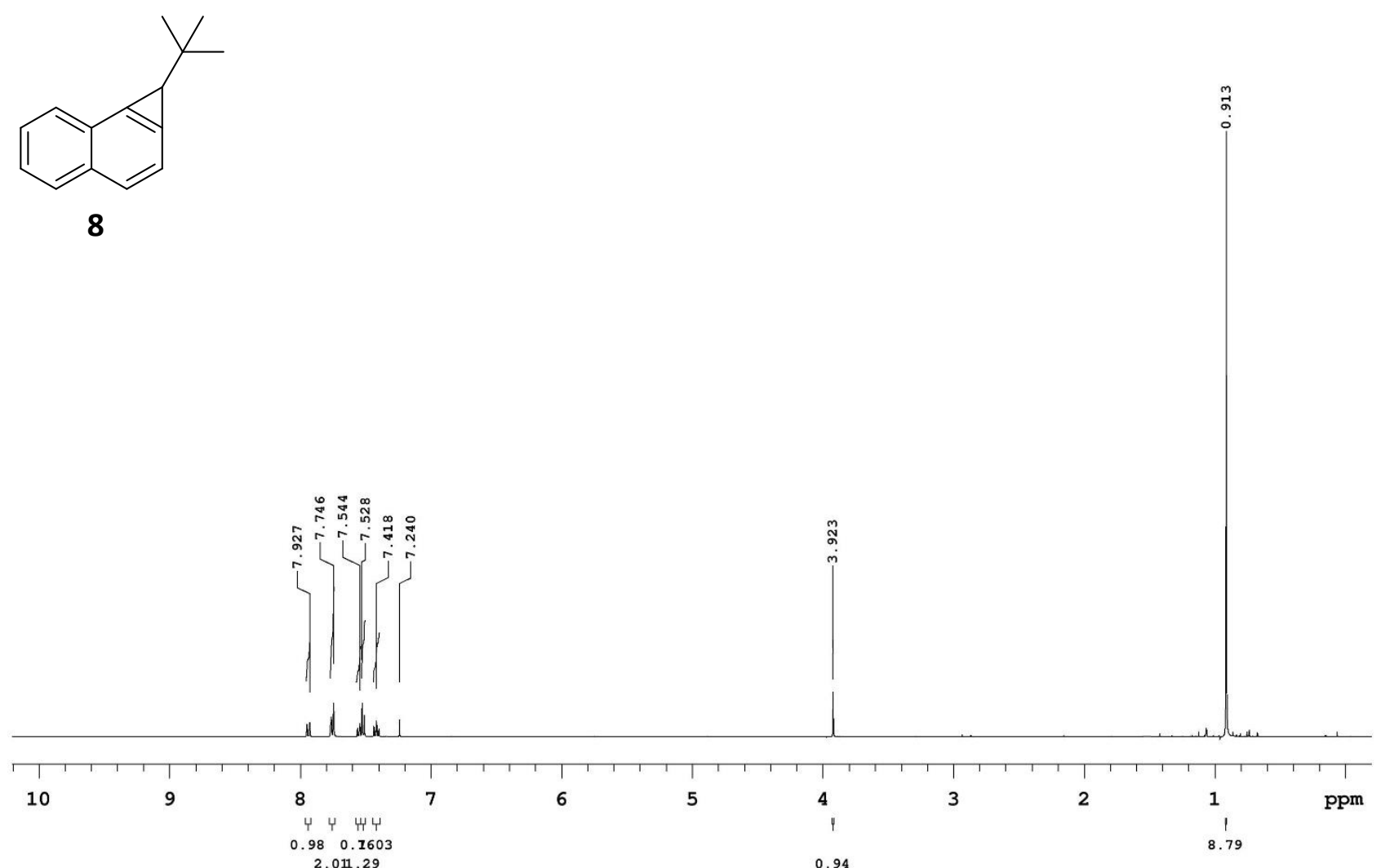

Sample Name: DA-256

Solvent: cdc13

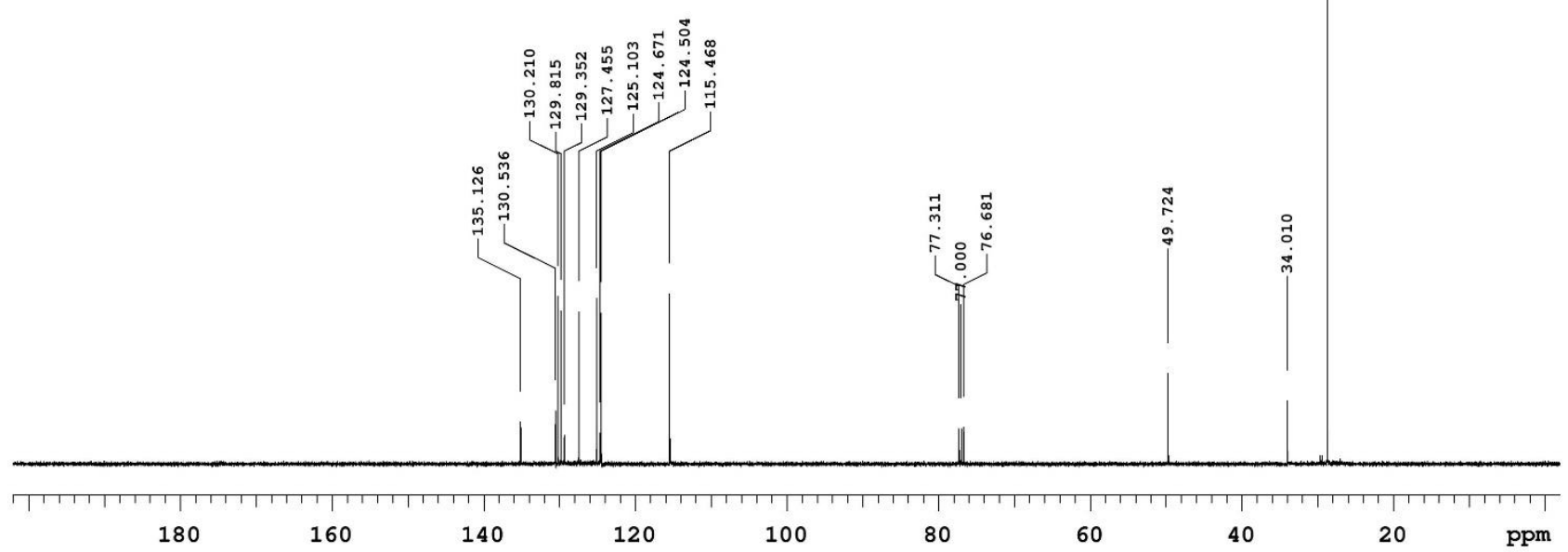

